\title{
Activation of Nitric Oxide and Dioxygen at Diferrous Complexes with Compartmental Pyrazolate Ligand Scaffolds
}

\author{
Dissertation \\ zur Erlangung des mathematisch-naturwissenschaftlichen Doktorgrades \\ "Doctor rerum naturalium" der Georg-August-Universität Göttingen \\ im Promotionsprogramm BioMetals \\ der Georg August University School of Science (GAUSS)
}

vorgelegt von

Anne Rebecca Schober aus Essen 


\section{Betreuungsausschuss}

Prof. Dr. Franc Meyer

Institut für Anorganische Chemie, Georg August Universität Göttingen

\section{Dr. Inke Siewert}

Institut für Anorganische Chemie, Georg August Universität Göttingen

Prof. Dr. Ebbe Nordlander

Lunds Universitet, Schweden

\section{Prüfungskomitee}

Referent: Prof. Dr. Franc Meyer

Institut für Anorganische Chemie, Georg August Universität Göttingen

Korreferentin: Dr. Inke Siewert

Institut für Anorganische Chemie, Georg August Universität Göttingen

Prof. Dr. Sven Schneider

Institut für Anorganische Chemie, Georg August Universität Göttingen

Prof. Dr. Dietmar Stalke

Institut für Anorganische Chemie, Georg August Universität Göttingen

Prof. Dr. Thomas Waitz

Institut für Anorganische Chemie, Georg August Universität Göttingen

\section{Dr. Franziska Thomas}

Institut für Organische und Biomolekulare Chemie, Georg August Universität Göttingen

Datum der mündlichen Prüfung: 18. August 2016 
1 Introduction 1

1.1 General Introduction . . . . . . . . . . . . . . . . . . . . . . . . . . 1

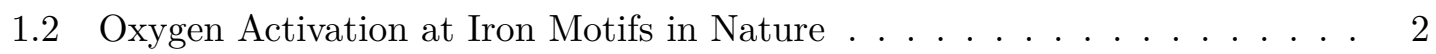

1.3 Oxygen Activation in Diiron Proteins $\ldots \ldots \ldots . . \ldots . . \ldots 3$

1.3 .1 Hemerythrin . . . . . . . . . . . . . . . . . . 4

1.3 .2 Soluble Methane Monooxygenase (sMMO) . . . . . . . . . . . . . . 4

1.3 .3 Arylamine Oxygenase . . . . . . . . . . . . . . . . . 6

1.3.4 Comparison of Diiron Peroxo Intermediates . . . . . . . . . . . . 7

1.4 Low Molecular Weight Analogues for Diiron-Oxygen Intermediates . . . . . 8

1.4 .1 Models for Hemerythrin . . . . . . . . . . . . . . . 8

1.4 .2 Models for sMMO . . . . . . . . . . . . . . . . . . 10

1.5 Nitric Oxide in Biological Systems . . . . . . . . . . . . . . . . . 13

1.6 The Enemark-Feltham Notation . . . . . . . . . . . . . . . . . . . . . . . . . 14

1.7 Nitric Oxide Reductases . . . . . . . . . . . . . . . . . . . . . . . 14

$1.7 .1 \quad$ Flavodiiron Proteins (FDPs) . . . . . . . . . . . . . . . . . . . . . . . . . 15

1.8 Functional Diiron Models for NO-Reduction . . . . . . . . . . . . . . . . 17

$\begin{array}{lll}2 \text { Aim of this Work } & 19\end{array}$

3 Design and Synthesis of Pyrazolate based Bis(pentadentate) Ligand Scaffolds 21

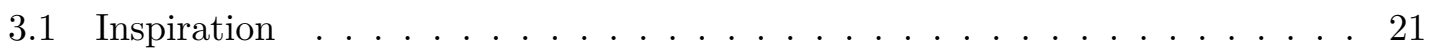

$3.2 \quad$ Anticipated Ligand Design . . . . . . . . . . . . . . . . . . . . . . . . . 23

3.3 Synthetic Strategy $\ldots \ldots \ldots \ldots$. . . . . . . . . . . . . . . . . . 23

3.3.1 Synthesis of the Pyrazole Building Unit . . . . . . . . . . . . . . 23

3.3 .2 Side Arms . . . . . . . . . . . . . . . . . . . . . . 25

$3.3 .3 \quad$ Synthesis of $\mathrm{HL}^{\mathrm{Im}} \ldots \ldots \ldots \ldots \ldots \ldots$

3.3 .4 Synthesis of $\mathrm{HL}^{\mathrm{Py}} \ldots \ldots \ldots \ldots \ldots . \ldots \ldots 26 \ldots \ldots \ldots$

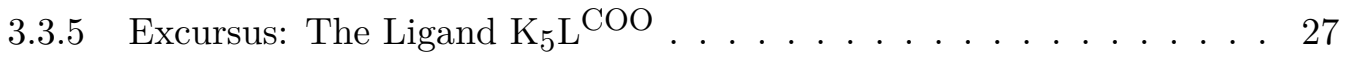

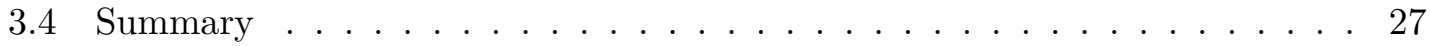

4 Synthesis and Characterization of Diferrous Complexes with Nitrile Coligands 29

4.1 Introduction . . . . . . . . . . . . . . . . . . . . . . 29

4.2 Synthesis and Structural Characterization of Diferrous Complexes with HL ${ }^{\mathrm{Im}} 30$

$4.2 .1 \quad$ ESI Mass Spectrometry $\ldots \ldots \ldots$. . . . . . . . . . . . 33

$4.2 .2 \quad$ Mössbauer Spectroscopy . . . . . . . . . . . . . . . . . . . . . 34 
4.2 .3 Anion Effects in the Solid State. . . . . . . . . . . . . . . . 34

\begin{tabular}{|lll}
\hline 4.3 & Spin Crossover of Diferrous Complexes of HL & Im \\
\end{tabular}

4.3 .1 SCO of 1a in Solid State . . . . . . . . . . . . . 36

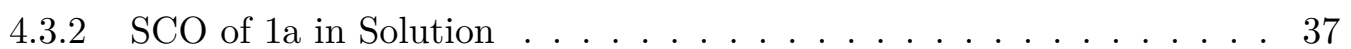

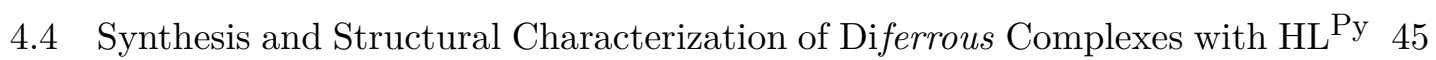

$4.4 .1 \quad$ ESI Mass Spectrometry . . . . . . . . . . . . . . . . . 47

$4.4 .2 \quad$ Mössbauer Spectroscopy and SQUID Magnetometry . . . . . . . . . 48

$4.4 .3 \quad$ Variable Temperature NMR and UV/Vis Spectroscopy. . . . . . . . 49

$4.4 .4 \quad$ Nitrile Exchange in Solution . . . . . . . . . . . . . . 50

4.5 Summary and Conclusions $\ldots \ldots \ldots \ldots \ldots \ldots$. . . . . . . . . . . 51

5 Reactivity of the Ditron Core with Dioxygen 53

5.1 Dioxygen Activation with the Diferrous Complex $1 . \ldots . . . . . .554$

5.1 .1 Oxidation in $\operatorname{Air} \ldots \ldots \ldots \ldots . \ldots . \ldots . \ldots 54$

5.1 .2 Reactivity with Dry Dioxygen . . . . . . . . . . . . . 56

$5.2 \quad$ Dioxygen Activation with the Diferrous Complex 2a. . . . . . . . . . . 60

$5.2 .1 \quad$ Reactivity with Dry Dioxygen . . . . . . . . . . . . . . . 60

5.2 .2 Reactivity with Oxygen Transfer Reagents . . . . . . . . . . . . . 62

5.3 Summary and Conclusions $\ldots \ldots \ldots \ldots \ldots$. . . . . . . . . . . 63

6 Diiron Dinitrosyl Complexes and their Reactivity upon Reduction 65

6.1 Preparation and Structural Characterization of $\left[\{\mathrm{Fe}(\mathrm{NO})\}^{7}\right]_{2}$ Complexes . . 66

$\left.6.1 .1 \quad\left[\mathrm{~L}^{\operatorname{Im}}\{\mathrm{Fe}(\mathrm{NO})\}_{2}\right](\mathrm{OTf})_{3}, \mathrm{MeOH}(4)\right] \ldots \ldots \ldots 66$

$\left.6.1 .2 \quad\left[\mathrm{~L}^{\mathrm{Py}}\{\mathrm{Fe}(\mathrm{NO})\}_{2}\right](\mathrm{OTf})_{3}(5)\right] \ldots \ldots \ldots \ldots \ldots$

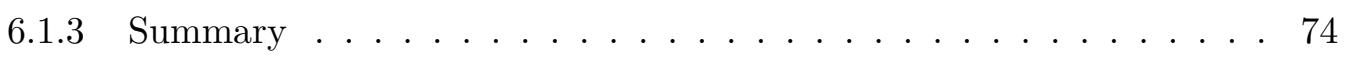

6.2 Electrochemical Properties and Reduction of Diiron Dinitrosyl Complexes . 75

$6.2 .1 \quad$ Cyclic Voltammetry and IR-Spectroelectrochemistry of 4 . . . . . 75

6.2 .2 Chemical Reduction of $4 \ldots \ldots \ldots \ldots 77$

$6.2 .3 \quad$ Cyclic Voltammetry and IR-Spectroelectrochemistry of 5 . . . . . 80

6.2 .4 Chemical Reduction of $5 \ldots \ldots \ldots$. . . . . . . . . . . . . . . . . . . . . . 81

$6.2 .5 \mathrm{~N}_{2} \mathrm{O}$ Detection $\ldots \ldots \ldots \ldots \ldots \ldots \ldots$

6.2 .6 Summary . . . . . . . . . . . . . . . . . 86

7 Excursus: Dinuclear Iron Complexes for Water Oxidation Catalysis 89

7.1 A First Dinuclear Iron Complex with the Ligand $\mathrm{L}^{\mathrm{COO}} \ldots . . . . . . . .993$

$7.2 \quad$ A Water Soluble Diiron Diaqua Complex . . . . . . . . . . . . . . . . 94

$7.3 \quad$ Analysis and Electrochemistry of a Tetranuclear Manganese Complex . . . 99

7.4 Summary . . . . . . . . . . . . . . . . . . . . . . . 103

\begin{tabular}{lll}
\hline & Summary and Conclusions & 105
\end{tabular}

\begin{tabular}{|lll}
\hline & Experimental & 107
\end{tabular}

9.1 Procedures and Methods . . . . . . . . . . . . . . . . . . . . . . . . . . 107

9.2 Synthesis of the Pyrazole Building Block . . . . . . . . . . . . . . . . . 111

9.3 Synthesis of the Ligands $\ldots \ldots \ldots$. . . . . . . . . . . . . . . . 114 
9.4 Complex Formation with Actetonitrile Coligands . . . . . . . . . . . . . . 115

9.5 Diiron Nitrosyl Complexes and their Reduction Products . . . . . . . . . 117

9.6 Complexes with $\mathrm{K}_{5} \mathrm{~L}^{\mathrm{COO}} \ldots \ldots \ldots \ldots \ldots \ldots \ldots$

$\begin{array}{ll}10 \text { Crystallographic Details } & 121\end{array}$

$\begin{array}{ll}11 \text { Appendix } & 137\end{array}$

11.1 Theory on Mössbauer Spectroscopy and SQUID Magnetometry . . . . . . . 137

11.1 .1 Mössbauer Spectroscopy . . . . . . . . . . . . . . . . . . 137

11.1 .2 SQUID Magnetometry . . . . . . . . . . . . . . . . . . . . . . 141

11.2 Supplementary Material for the Characterization of the Diferrous Complexes 144

11.2 .1 NMR and UV/Vis Studies of $2 \mathrm{a} \ldots \ldots . \ldots 144$

11.2 .2 ESI Mass Spectra $\ldots \ldots \ldots$. . . . . . . . . . . . . . . . 145

11.2 .3 Mössbauer spectra $\ldots \ldots \ldots$. . . . . . . . . . . . . . . . 145

11.3 Supplementary Material for the Reactivity Studies of the Diiron Core with

Dioxygen . . . . . . . . . . . . . . . . . . . . . . 148

11.4 Supplementary Material for the Characterization of Diiron Dinitrosyl Com-

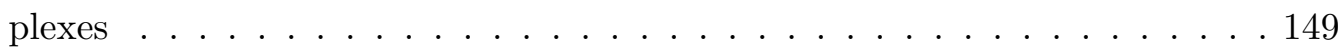

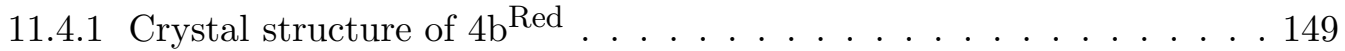

11.4 .2 IR Spectrum of $4^{\text {Red }} \ldots \ldots$. . . . . . . . . . . . . . . . . . . . . . . . . . . . . . . . . . . . . . . . . .

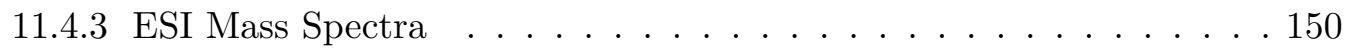

11.4 .4 Labelling Studies with 4 . . . . . . . . . . . . . . . . . . . . . . . . . . . . . . . . . . . . . . . . . . .

$11.4 .5 \mathrm{UV} / \mathrm{Vis}$ Spectra of $4 \ldots \ldots \ldots 151$

$11.4 .6 \mathrm{UV} /$ Vis Spectra of Cobaltocene . . . . . . . . . . . . . . . 152

11.4 .7 Setup for IR Headspace Analyses . . . . . . . . . . . . . . . . . . 152

11.5 Supplementary Material for the Excursus on Water Oxidation Catalysis. . . 153

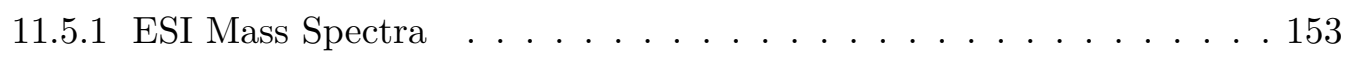

11.6 Abbreviations . . . . . . . . . . . . . . . . . . . . . 154

11.7 Overview over Synthesized Complexes . . . . . . . . . . . . . 156

\begin{tabular}{ll}
\hline Bibliography & 157
\end{tabular} 



\section{Introduction}

\subsection{General Introduction}

The element iron is a group 8 transition metal and plays a crucial role in nature. With a natural abundance of $4.7 \mathrm{wt} \%$ it is the fourth most frequent metal in the geosphere. 1] Iron is an important trace element in the human body and is essential among other functions for oxygen transport and thus respiration. ${ }^{2}$ Its common oxidation states are + II and + III. $\underline{3}$. As a first row transition metal iron in these oxidation states can readily switch between high spin and low spin states, or as in the case of iron (III), even maintain an intermediate spin state. 4. This is beneficial for the manifold reactivity that can be observed in iron chemistry. ${ }^{4}$

Enzymes that contain iron in their active site are widely recognized in biology. The family of iron proteins is extremely large as is the range of their functions. Not only can these proteins mediate redox processes or substrate binding, they are often powerful catalysts under physiological conditions. [5] Synthetic chemistry can extremely benefit from a deeper understanding of these functions and their structural and spectroscopic characterization. The optimization of catalytic reactions on a greater scale is a challenging process, to which a broader knowledge through the synthesis of low molecular weight analogues, that mimic the enzymes' active sites, can considerably contribute. ${ }^{6 \mid 7]}$ Low molecular weight analogues thus serve as very helpful tools in mimicking intermediates in enzymatic catalysis as well as substrate binding and activation. 8 ]

Small molecules, e.g. $\mathrm{O}_{2}, \mathrm{~N}_{2}$, and NO, if activated in a controlled way by enzymes or transition metal complexes, may serve as synthons to build more complex molecules. Moreover, they often serve as signaling agents which trigger important protein expression and regulation processes. 9 Since small molecules are often thermodynamically quite stable, for their successful use kinetic barriers have to overcome. Transition metals such as in diiron complexes in this work have been known to provide alternative reaction pathways with lower energy barriers by binding and transformation of these small molecules. ${ }^{9}$.

This work will in particular address binding and activation of dioxygen and nitric oxide at diiron sites. In mammals binding of dioxygen to iron sites and accompanying oxygenation reactions are considered fundamental for life. ${ }^{8}$ Iron sites are involved in a multitude of 
processes such as, among others, oxygen transport, oxygen storage and important redox reactions.

Nitric oxide binding to iron centers, however, has been widely underestimated for a long time. Nitric oxide displays a key role in nature as a signaling molecule in organisms and can act in cytotoxic and cytoprotective ways. 10.

\subsection{Oxygen Activation at Iron Motifs in Nature}

The controlled activation of dioxygen in nature is very challenging. The reduction of molecular oxygen with subsequent substrate oxygenation initially requires four electrons which have to be provided by the enzyme. Enzymes that mediate the oxygenation of substrates can be generally divided into mono- and dinuclear oxygenases. 11 Mononuclear oxygenases can be subdivided into heme and non heme oxygenases.

Heme in general can be described as a protoporphyrin IX scaffold with an iron atom in its macrocyclic binding pocket. Heme serves as prosthetic group of a variety of hemoproteins such as hemoglobin, myoglobin, cytochromes, guanylyl cyclase and nitric oxide synthase. 12] Many heme oxygenases that are discussed to perform substrate oxygenation derive from the cytochrome-P450 family. The reaction sequence in their catalytic cycle involves a sequential two electron reduction of dioxygen coupled with protonation steps and heterolytic bond cleavage of the oxygen-oxygen bond. 13] A water molecule is released in the course this event. High valent iron peroxo and oxo intermediates are postulated for the catalysis in which oxidation states up to Fe(IV) are expected for the central iron atom. 14

Mononuclear non heme iron oxygenases differ from heme oxygenases structurally and spectroscopically. While the porphyrin macrocycle is almost planar in hemes and leaves a vacant axial site for dioxygen binding, mononuclear non heme iron enzymes feature a variety of structural motifs. 15 A recurring structural similarity in these iron oxygenases is the 2-His-1-carboxylate facial triad. [16] The metal center is five- or sixfold coordinated. Three coordination sites are filled by two histidine amino acids and a carboxylate from either a glutamate or aspartate residue. Additional coordination sites at the central iron atom are vacant or occupied by weakly bound solvent molecules. $16[17$ Substrate and oxygen binding occurs at these sites opposed to the triad motif. [18] The ladder of oxidation states of the central iron atom during the catalytic cycle of these enzymes ranges from $\mathrm{Fe}(\mathrm{II})$ to Fe(IV). From theoretical calculations even $\mathrm{Fe}(\mathrm{V})$-oxo-hydroxo intermediates have been hypothesized. 19 .

However, this work will focus on the structural and spectroscopic diversity of dinuclear non heme iron proteins and their ability to activate small substrates. In analogy to mononuclear non heme iron oxygenases, dinuclear non heme iron oxygenases contain a diiron core stabilized by the amino acids histidine, glutamate and aspartate. 20] High valent intermediates of the diiron core have been spectroscopically observed during the process of oxidation catalysis.

A brief overview of iron-oxygen intermediates in the respective enzymes is given in Scheme 1.1 


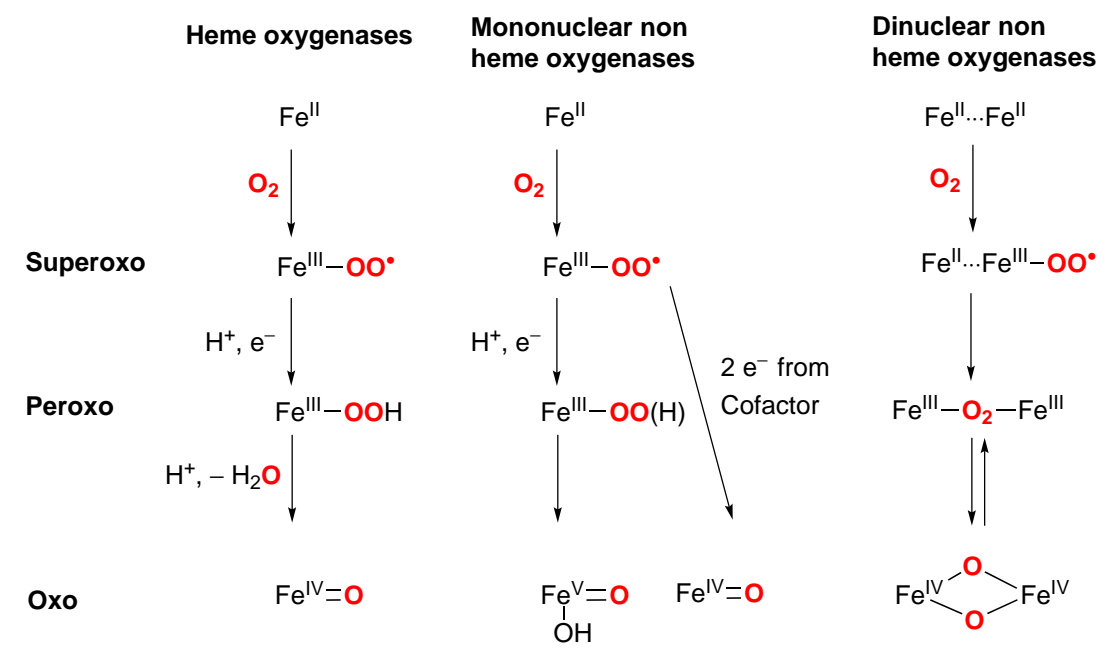

Scheme 1.1: Overview: Intermediates in the activation of dioxygen by iron oxygenases. [5]

In the following section the family of diiron proteins in the context of oxygen activation will be discussed more closely.

\subsection{Oxygen Activation in Diiron Proteins}

Non heme diiron proteins have gained increasing recognition over the last decades and have been intensely studied with regard to their structure and function. 20121] Most of these enzymes share a common structural motif which comprises a carboxylate bridged diiron core as well as carboxylic and nitrogen donating amino acids, in particular glutamate, aspartate and histidine. 20122] This non heme diiron superfamily is involved in a variety of versatile functions among others iron storage in ferritin, oxygen transport in hemerythrin, radical generation in ribonucleotide reductase and hydrocarbon oxidation in multicomponent monooxygenases. 23 The reversible binding and/or activation of dioxygen resembles a key role in the functions of many of these enzymes. Examples of how oxygen can bind to a dinuclear iron center are illustrated in Scheme 1.2 .

$$
\begin{aligned}
& \mathrm{Fe}^{-}{ }_{\mathrm{O}}^{\mathrm{O}} \mathrm{Fe} \quad \mathrm{Fe}^{-\mathrm{O}_{-}-\mathrm{Fe}} \\
& \eta^{1} \quad \text { trans }-\mu-\eta^{1}: \eta^{1} \text {-peroxo }
\end{aligned}
$$

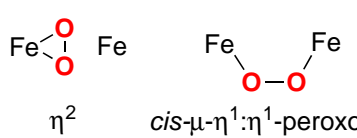<smiles>OP([18F])[18F]</smiles>$$
\eta^{1}-1,1-\text { peroxo }
$$$$
\mathrm{Fe}^{-} \stackrel{\mathrm{O}}{\mathrm{O}}-\mathrm{Fe}
$$$$
\begin{aligned}
& \mathrm{Fe}^{\prime}, \mathrm{I}_{\mathrm{O}}^{\prime}, \mathrm{Fe} \\
& \mu-\eta^{2}: \eta^{2} \text {-peroxo }
\end{aligned}
$$$$
\eta^{1}: \eta^{2}-1,2 \text { peroxo }
$$$$
\mathrm{Fe}_{-\mathrm{O}}^{-\mathrm{O}} \mathrm{-Fe}
$$$$
\operatorname{bis}(\mu-\mathrm{oxo})
$$

Scheme 1.2: Possible binding modes of dioxygen to diiron sites. 24

A deeper understanding of the function of the diiron core in a particular enzyme can be beneficial in many ways as enzymes operate under physiological conditions and often facilitate energy consuming reactions by lowering activation barriers significantly.

In the following section three representatives, hemerythrin, soluble methane monooxygenase and arylamine oxygenase, of the non heme diiron family with distinct oxygen binding 
modes will be discussed in more detail.

\subsubsection{Hemerythrin}

The diiron core in hemerythrin, found in several marine invertebrates, is capable to reversibly bind dioxygen. Thus the enzymes' main function lies in oxygen transport. Hemerythrin has been one of the first structurally characterized non heme diiron centers. Intriguingly, apart from most other non heme diiron proteins this carrier protein is stabilized by five terminal histidine residues instead of the common two terminal histidines. 21] Two bridging carboxylates provided by aspartate and glutamate as well as a bridging hydroxo ligand complete the coordination sphere of the diiron core. ${ }^{[22[25]}$ The Fe...Fe distance lies in the range of 3.25 to $3.5 \AA$. 25 .
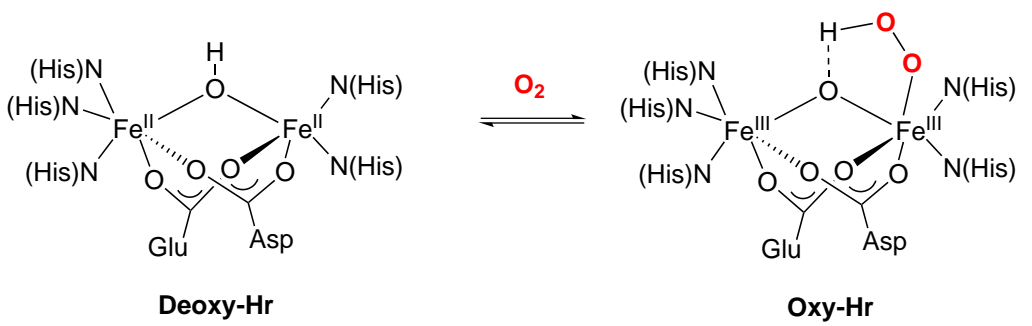

Scheme 1.3: Reversible oxygen binding in hemerythrin. 21

The binding of dioxygen in hemerythrin is reversible as depicted in Scheme 1.3. Through the terminal binding of oxygen to one iron center a hydroperoxo species is formed in junction with the bridging hydroxide. Two electrons are transferred from the diiron(II) core to the oxygen molecule to form a diiron(III) species. ${ }^{20}$. The diiron hydroperoxo unit exhibits very specific spectroscopic features, e.g., a UV/Vis band at $\sim 500 \mathrm{~nm}$ attributed to a charge transfer from the peroxo unit to $\mathrm{Fe}(\mathrm{III})$ and a Raman signal at $844 \mathrm{~cm}^{-1}$. 20126 Moreover the two iron sites differ in their coordination sphere which is reflected in two quadrupole doublets in the Mössbauer spectrum. $\left(\delta=0.51\right.$ and $0.52 \mathrm{~mm} \mathrm{~s}^{-1},\left|\Delta E_{\mathrm{Q}}\right|=$ 1.96 and $\left.0.95 \mathrm{~mm} \mathrm{~s}^{-1}\right) .202728$

\subsubsection{Soluble Methane Monooxygenase (sMMO)}

The soluble methane monooxygenase portrays one of the most recognized examples among the superfamily of diiron proteins. Its unique ability to oxidize, among other substrates, the inert hydrocarbon methane to methanol has drawn a lot of attention toward the function of this enzyme. sMMO occurs in several methanotrophic bacteria. First structurally characterized in 1993 from Methylococcus capsulatus (Bath), the enzyme consists of three components: a hydroxylase (MMOH), an NADH oxidoreductase (MMOR) and a regulatory protein (MMOB). 29] 31 For its distinct function all three components are essential.

The hydroxylase is a $251 \mathrm{kDa}$ heart-shaped hetero-dimer consisting of two $\alpha \beta \gamma$ protomers. 31 The diiron active site is located in a four-helix bundle in each of the $\alpha$ subunits of the hydroxylase. 3132 The diiron core in sMMO is coordinated by two terminal histidines and in total four carboxylates provided by glutamates. $21 / 32$ The coordination 
sphere of the reduced diferrous state is completed by solvent molecules, mainly water. In the diferric resting state of sMMO two hydroxide ligands form bridges between the two iron sites. Scheme 1.4 shows a representation of the two described forms.
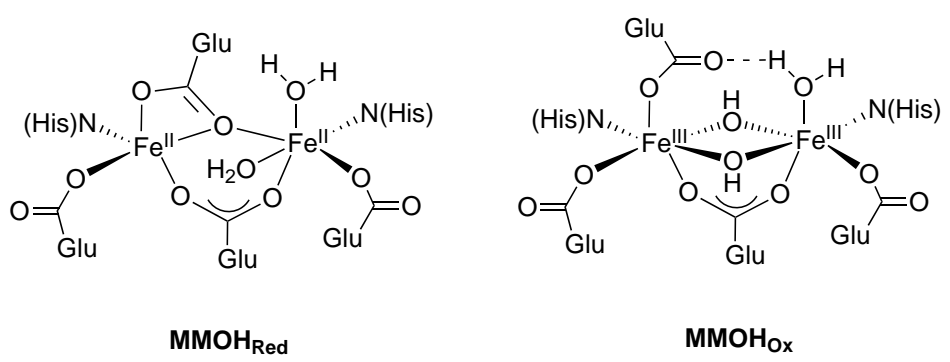

Scheme 1.4: Diferrous state (left) and diferric resting state (right) of the diiron center in sMMO. 21]

In $\mathbf{M M O H}_{\mathbf{R e d}}$ the distance of the two $\mathrm{Fe}(\mathrm{II})$ ions lies in the range of 3.2 to $3.5 \AA$. 30 This distance shortens in $\mathbf{M M O H}_{\mathbf{O x}}$ to $3.1 \AA$ due to the exogenous hydoxide bridging ligands. 29]30] While a ferromagnetic ground state has been suggested for $\mathbf{M M O H} \mathbf{H e d}_{\mathbf{R e d}}$, the two $\mathrm{Fe}(\mathrm{III})$ ions in $\mathbf{M M O H}_{\mathbf{O x}}$ are antiferromagnetically coupled. ${ }^{33}$ Here the bridging hydroxide ligands mediate the spin exchange. ${ }^{32}$ The overall reaction sequence in sMMO basically involves protons, electrons, dioxygen and a hydrocarbon to yield the oxygenated hydrocarbon and water. 34

$$
2 \mathrm{H}^{+}+2 \mathrm{e}^{-}+\mathrm{O}_{2}+\mathrm{CH}_{4} \longrightarrow \mathrm{CH}_{3} \mathrm{OH}+\mathrm{H}_{2} \mathrm{O}
$$

Electrons are provided by the reductase component (MMOR), which is responsible for the reduction of the diiron core to its diferrous form. This step is realized by the transfer of two electrons from NADH through the flavin adenine dinucleotide cofactor of MMOR and its $\left[\mathrm{Fe}_{2} \mathrm{~S}_{2}\right]$ cluster. ${ }^{34}$ The regulatory protein MMOB is involved in structural rearrangements of the active site during the catalytic cycle and mediates the inlet of gases and substrates. Recent studies attribute this ability of MMOB to an $N$-terminal tail of the regulatory protein that has been structurally resolved and seems to be unique within the non heme diiron family. ${ }^{34] 35}$ The catalytic cycle of sMMO involves several steps which have been elucidated spectroscopically over the last decade. Scheme 1.5 depicts a catalytic cycle as proposed by Proshlyakov and Lipscomb. In a first step dioxygen reacts with the $\mathbf{M M O H}_{\text {Red }}$ site in a reductive addition, possibly via a superoxide species $\mathbf{P}^{*}$, to yield a peroxo-diiron(III) core $\mathbf{P}$. Before the formation of $\mathbf{P}^{*}$ a putative intermediate $\mathbf{O}$ has been proposed, which has dioxygen bound to the enzyme but not necessarily to the diiron core. 36

$\mathbf{P}$ has been spectroscopically fully characterized, however structurally the binding mode of $\mathrm{O}_{2}$ has not been identified. Studies suggest a $\mu$-1,2-dinuclear peroxo complex. $\mathbf{P}$ decays to form a high valent intermediate $\mathbf{Q}$. $\mathbf{Q}$ can be assigned with high probability to a closed shell bis- $\mu$-oxo diiron(IV) species from very recent Raman studies. ${ }^{37}$ In these experiments $\mathrm{Q}$ is generated with natural abundant and ${ }^{18} \mathrm{O}$ labeled dioxygen. In comparison with Raman data of synthetic models, an open shell diiron core can be ruled out due to very different spectroscopic signatures. These studies moreover suggest that both oxygen atoms 


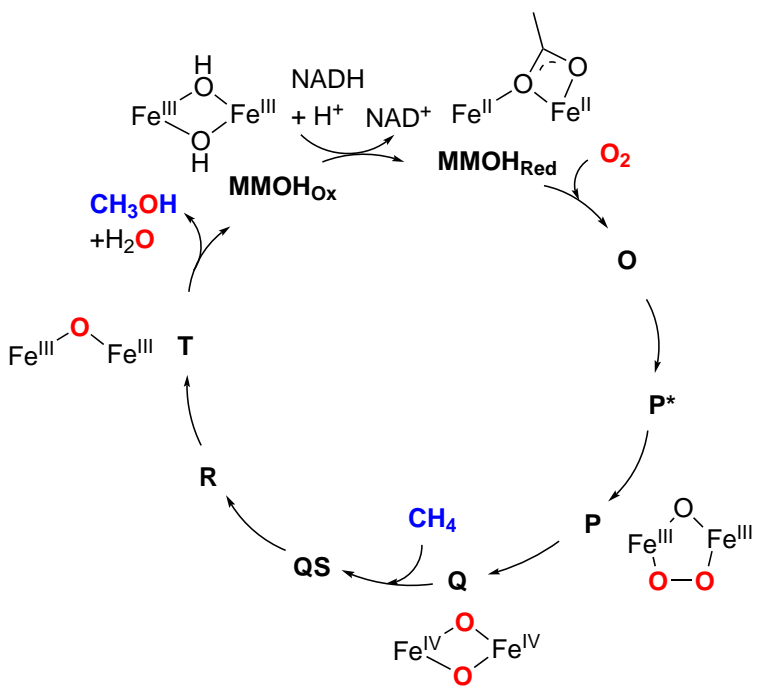

Scheme 1.5: Proposed catalytic cycle of sMMO with most investigated intermediates. $\underline{36 \mid 37}$

in $\mathbf{Q}$ originate from dioxygen. In a subsequent step methane is added to $\mathbf{Q}$. The reaction pathway proceeds via the transient intermediates $\mathbf{Q S}$ and $\mathbf{R}$ to yield the product complex $\mathbf{T}$ and the oxygenated hydrocarbon. $\mathbf{T}$ is presumably a singly $\mu$-oxo bridged diiron species. The oxo bridge is assumed to originate from the incorporated dioxygen molecule. $\frac{37}{3}$. The transformation of $\mathbf{T}$ to the $\mathbf{M M O H} \mathbf{O}_{\mathbf{O x}}$ resting state leads to the release of methanol and a water molecule.

\subsubsection{Arylamine Oxygenase}

As a third example the oxygen binding in arylamine oxygenases will be shortly described. Arylamine oxygenases in general catalyze the six electron reduction of an amine to a nitro group through the activation of dioxygen by a non heme diiron center. These reactions occur in the biosynthesis of antibiotics such as, among several others, aureothin by the enzyme $p$-aminobenzoate $N$-oxygenase $(\mathrm{AurF})$ or chloramphenicol by the enzyme chloramphenicol $N$-oxygenase $(\mathrm{CmII})$. 38] A first crystal structure of a arylamine oxygenase from streptomyces thioluteus in its oxidized state was published in 2008. ${ }^{39}$. The diiron core in this class of enzymes differs from the active site of sMMO in the exchange of one water ligand for an extra histidine. Thus, the two iron centers are coordinated by three terminal histidines and in total four carboxylates. Intriguingly, this class of enzymes seems to exhibit a different binding mode than observed for other non heme diiron proteins upon the addition of dioxygen to the reduced diferrous state. First UV/Vis and Mössbauer studies by Bollinger and Krebs verified a peroxo-Fe(III)Fe(III) state with unusually blue shifted absorption bands in the UV/Vis range. 40] These findings were lately confirmed by additional experiments including Raman measurements. 38 . All collected data to date point to a different binding mode of dioxygen in the active site. QUE and LIPSCOMB propose a $\mu-\eta^{1}: \eta^{2}$-peroxo ligand to accout for these distinct spectroscopic features. 38 . The mechanism of substrate oxygenation and reaction pathways of amine oxygenation are to date not fully elucidated. A possible sequence for the oxygenation of an amine to the respective nitro-group was proposed by QUE and LIPSCOMB in 2015. 38 In this scenario 


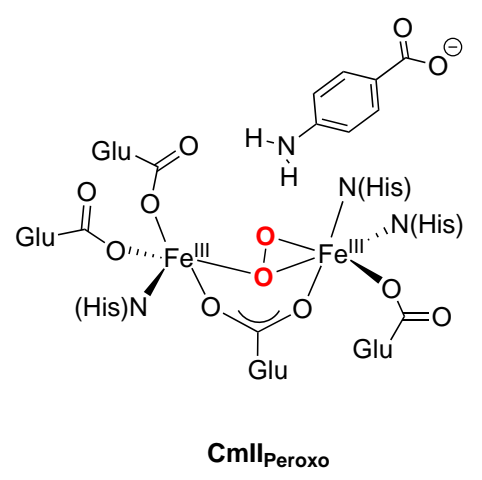

Scheme 1.6: Proposed peroxo diiron center involved in arylamine oxygenation of chloramphenicol. 38

the amine $\left(\mathrm{Ar}-\mathrm{NH}_{2}\right)$ can transfer an electron onto the peroxide moiety yielding a mixed valent $\mathrm{Fe}(\mathrm{III}) \mathrm{Fe}(\mathrm{IV})$ oxo( $\mu$-oxo) diiron core. Subsequent proton transfer would give rise to a $\mathrm{Fe}(\mathrm{III}) \mathrm{Fe}(\mathrm{IV})$ hydroxo( $\mu$-oxo) species and a deprotonated amine radical. Homolytic $\mathrm{Fe}-\mathrm{OH}$ bond scission and $\mathrm{N}-\mathrm{O}$ bond formation would yield a hydroxylamine and a $\mathrm{Fe}(\mathrm{III}) \mathrm{Fe}(\mathrm{III})$ oxo core. A second reaction with another equivalent of the diiron-peroxo species would finally yield the nitro group. 41] The described reaction sequence is depicted in Scheme 1.7 .

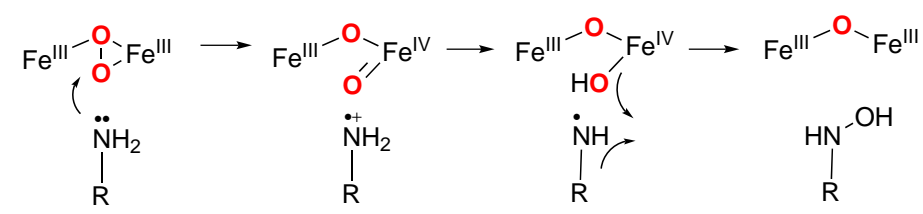

Scheme 1.7: First reaction sequence for the oxygenation of arylamines proposed by QuE and LIPSCOMB.

However, this proposal contrasts findings by Bollinger and KreBs from 2010, who describe the mechanism as a nucleophilic attack of the amine and proton transfer to the diiron core. ${ }^{42}$ Conclusive evidence for a reaction pathway with structurally characterized intermediates has not been described to date.

\subsubsection{Comparison of Diiron Peroxo Intermediates}

The three enzymes described in this section were chosen for their very different binding modes of dioxygen to a non heme diiron center. Scheme 1.8 compares the structural motifs, which are proposed to occur upon the addition of dioxygen to the reduced diiron(II) site. Table 1.1 gives an overview of characteristic spectroscopic features which are attributed to these diiron oxygen intermediates. 


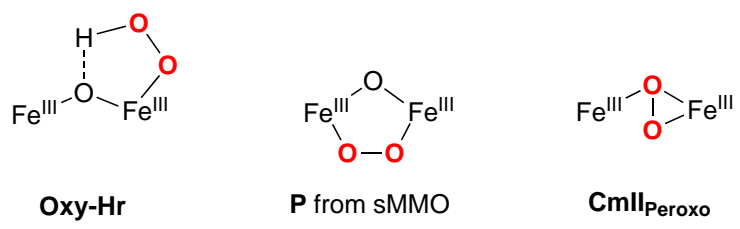

Scheme 1.8: Comparison of oxygen binding in the three examples of non heme diiron proteins described in the text.

Table 1.1: Overview of characteristic spectroscopic features of peroxo intermediates of the described enzymes.

\begin{tabular}{|c|c|c|c|c|}
\hline & $\begin{array}{l}\mathrm{UV} / \mathrm{Vis} \\
\lambda(\epsilon) \\
{[\mathrm{nm}]\left(\left[\mathrm{M}^{-1} \mathrm{~cm}^{-1}\right]\right)}\end{array}$ & $\begin{array}{l}\mathrm{MB} \\
\delta \\
{\left[\mathrm{mm} \mathrm{s}^{-1}\right]} \\
\end{array}$ & $\begin{array}{l}\left|\Delta E_{\mathrm{Q}}\right| \\
{\left[\mathrm{mm} \mathrm{s}^{-1}\right]}\end{array}$ & $\begin{array}{l}\text { Raman } \\
\tilde{\nu}(\mathrm{O}-\mathrm{O}) \\
{\left[\mathrm{cm}^{-1}\right]}\end{array}$ \\
\hline Oxy-Hr $20|26-28| 43]$ & $500(2200)$ & $0.50,0.51$ & $2.02,1.01$ & 844 \\
\hline $\mathbf{P}(\mathrm{sMMO})$ 44 45 & $725(1800)$ & 0.69 & 1.55 & $905^{\mathrm{a}}$ \\
\hline CmII $_{\text {Peroxo }} 38$ & $500(500)$ & $0.62,0.54$ & $-0.23^{\mathrm{b}},-0.68^{\mathrm{b}}$ & 741 \\
\hline
\end{tabular}

${ }^{\text {a }}$ Spectra were not reproducible. 46

${ }^{\mathrm{b}}$ Values for assumption of nested doublets. Sign of $\Delta E_{\mathrm{Q}}$ unknown. 38

\subsection{Low Molecular Weight Analogues for Diiron-Oxygen Intermediates}

Low molecular weight analogues may contribute to understanding characteristic features of an enzyme. A division can be made between structural and functional models. Structural models mostly mimic the active site of an enzyme with close conformity of ligands and/or coordination geometry of metal and substrate. Functional models, however, are developed to mimic the reactivity of the active site or a certain process within the enzyme. To date the majority of model systems in synthetic diiron-oxygen chemistry aim for the structural aspect of dioxygen binding to the diiron center. The library of ligands that have been used to realize this goal is very large. A few selected examples will be presented in the following section. As this work is inherently based on nitrogen donor ligands, examples that comprise this ligand environment were chosen. Complexes with nitrogen donor ligands may not always feature the same electronic spin states and match the enzymes' spectroscopic signatures entirely, but they are known to impart high stability even to high valent metal complexes as demonstrated by the successful isolation of numerous reactive intermediates supported by chelating ligands with nitrogen donor sites. 47 .

\subsubsection{Models for Hemerythrin}

The first models to mimic the dioxgen reactivity of hemerythrin were developed in the early 1980's. As hemerythrin was one of the first non heme diiron enzymes to be discovered, synthetic chemistry focused on designing model systems with similar spectroscopic features. These first complexes by WiEghardT and Lippard employed $N$ capping ligands such as trispyrazolylborate $\left(\mathrm{Bpz}_{3}\right)$ and $N, N^{\prime}, N^{\prime \prime}$-trimethyl-1,4,7-triazacyclononane (tacn) and bridging carboxylates to emulate the active site of hemerythrin. 48,52 As hemerythrin 
differs from other enzymes of the non heme diiron family because of its exceptionally nitrogen rich donor environment, these ligands seem to be well suited to mimic the active site. 21] With a Mestacn system both, a diferrous $(\mathbf{I})$ and a diferric model $\left(\mathbf{I}^{\mathbf{O}}\right)$ have been structurally characterized. [52] Systems that use dinucleating ligands to mimic the active site of hemerythrin have also been developed. One example is the 1,8-naphthyridine-based ligand BPEAN developed by the LIPPARD group. [53] The diferrous form of the diiron complex (II) exhibits upon oxygenation very similar spectroscopic features as observed for oxy-hemerythrin. Scheme 1.9 summarizes the described models for oxy-hemerythrin with their respective ligands. A detailed comparison of structural and spectroscopic parameters of the respective model with the $\mathbf{O x y}$ - and Deoxy-form of hemerythrin is given in Table 1.2 .
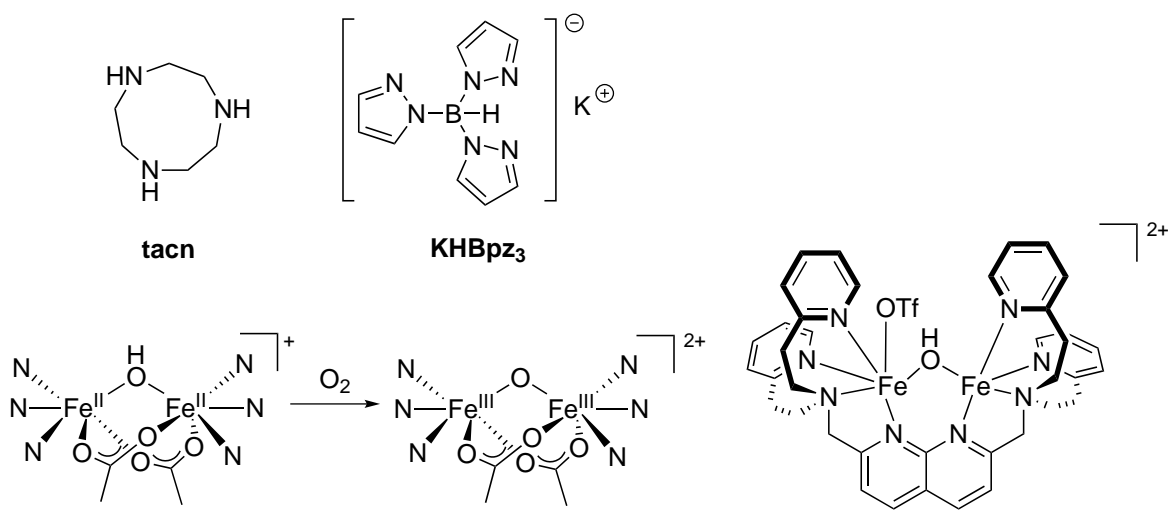

I

jox

II

Scheme 1.9: Top: Tacn and $\mathrm{HBpz}_{3}$ capping ligands employed in the first structural models for hemerythrin. $48 \mid 54$ Bottom: Schematic representation of the first diferrous model (I) with methyltacn ligands for methemeryrhin with the respective ferric form $\left(\mathbf{I}^{\mathbf{O x}}\right) \underline{52}$ and a diferrous complex of the dinucleating BPEAN ligand system that exhibits upon oxygenation spectroscopic features very similar to those found for oxy-hemerythrin. 53

Table 1.2: Comparison of structural and spectroscopic parameters of model complexes and enzyme.

\begin{tabular}{|c|c|c|c|c|c|}
\hline & $\begin{array}{l}\text { Deoxy- } \\
\text { Hr }{ }^{25|28| 55]}\end{array}$ & $\begin{array}{l}\text { Oxy- } \\
\text { Hr }{ }^{20|25| 28|43| 55]}\end{array}$ & $\mathbf{I} 52$ & $\mathrm{I}^{\mathrm{Ox}}[52]$ & $\mathrm{II}^{\mathrm{Ox}}[53$ \\
\hline $\mathrm{Fe} \cdots \mathrm{Fe}[\AA]$ & 3.32 & 3.27 & 3.32 & 3.12 & 3.53 \\
\hline$\lambda[\mathrm{nm}]$ & - & 500 & - & - & 505 \\
\hline$\epsilon\left[\mathrm{M}^{-1} \mathrm{~cm}^{-1}\right]$ & - & 2200 & - & - & 1500 \\
\hline$\delta\left[\mathrm{mm} \mathrm{s}^{-1}\right]$ & $1.15,0.54$ & $0.50,0.51$ & 1.15 & 0.47 & $0.50,0.51$ \\
\hline$\left|\Delta E_{\mathrm{Q}}\right|\left[\mathrm{mm} \mathrm{s}^{-1}\right]$ & $2.70,1.80$ & $2.02,1.01$ & 2.76 & 1.50 & $1.11,1.78$ \\
\hline$\tilde{\nu}(\mathrm{O}-\mathrm{O})\left[\mathrm{cm}^{-1}\right]$ & - & 844 & - & - & 868 \\
\hline
\end{tabular}




\subsubsection{Models for sMMO}

\section{Models for the Intermediate P}

As the sMMO has been thoroughly studied over many years, many models were developed to mimic intermediates of the enzymes' catalytic cycle. Despite all efforts and progress the stabilization and structural verification of such intermediates is still challenging. For the peroxo intermediate $\mathbf{P}$ merely five crystal structures aside from many spectroscopic studies exist to date that illustrate a peroxo-diiron core. [56] All of these peroxo-models have dioxygen bound to the diferric center in a cis- $\mu-1,2$-peroxo binding mode. The first structural model of a diiron core was presented in 1996 by SUZUKI and coworkers. $\frac{57}{}$. The diiron core was stabilized by a dinucleating ligand HPh-bimp (III). Interestingly, the binding of dioxygen could be reversed when boiling the complex in acetonitrile under a dinitrogen atmosphere. Shortly after, QUE and coworkers isolated a similar peroxo-diiron complex (IV) with the ligand system N-Et-hptb and triphenylphosphine oxide coligands at temperatures of $-40{ }^{\circ} \mathrm{C} .[58$ Also the LIPPARD group presented their first cis- $\mu$-1,2-peroxo complex $(\mathbf{V})$ obtained with a sterically more demanding version of the previously described trispyrazolylborate capping ligands in the same year. ${ }^{59}$. Other than in the two previously described peroxo-diiron models, their system featured a significantly larger dihedral angle of the Fe1-O1-O2-Fe2 unit and thus slightly different spectroscopic properties which on the other hand matched very well with spectroscopic signatures of the intermediate $\mathbf{P}$.

A couple of years later in 2005 SUZUKI and coworkers developed another pair of diironperoxo model complexes (VI) with tridentate pyridine/carboxylate hybrid ligands $\left(6 \mathrm{Me}_{2}-\right.$ $\mathrm{BPP}$. [60] Aside from the cis- $\mu-1,2$-peroxo bridge, the diiron core in these complexes is either bridged by an additional hydroxo or oxo ligand. Detailed spectroscopic studies are available for these two species. Scheme 1.10 depicts the described models for the intermediate $\mathbf{P}$ of sMMO. In Table 1.3 selected structural and spectroscopic parameters of the described models are compared.

Table 1.3: Comparison of spectroscopic and structural parameters of the intermediate $\mathbf{P}$ with respective model systems.

\begin{tabular}{l|llllll}
\hline & $\mathbf{P}^{[44 \mid 45]}$ & $\mathbf{I I I}^{[57}$ & $\mathbf{I V}^{[58[6162}$ & $\mathbf{V}^{[59]}$ & $\mathbf{V I}^{\mathrm{OH}}[\mathbf{6 0}$ & $\mathbf{V I}^{\mathrm{O}[60]}$ \\
\hline $\mathrm{Fe} \cdots \mathrm{Fe}[\AA]$ & - & 3.33 & 3.5 & 4.00 & 3.40 & 3.17 \\
$\mathrm{O}-\mathrm{O}[\AA]$ & - & 1.43 & 1.42 & 1.41 & 1.40 & 1.41 \\
$\lambda[\mathrm{nm}]$ & 725 & $500-800$ & 588 & 694 & 644 & 577 \\
$\epsilon\left[\mathrm{M}^{-1} \mathrm{~cm}^{-1}\right]$ & 1800 & 1700 & 1500 & 2650 & 3000 & 1500 \\
$\delta\left[\mathrm{mm} \mathrm{s}^{-1}\right]$ & 0.69 & $0.58,0.65$ & 0.52 & 0.66 & 0.50 & 0.50 \\
$\left|\Delta E_{\mathrm{Q}}\right|\left[\mathrm{mm} \mathrm{s}^{-1}\right]$ & 1.55 & $0.74,1.70$ & 0.72 & 1.40 & 1.31 & 1.46 \\
$\tilde{\nu}(\mathrm{O}-\mathrm{O})\left[\mathrm{cm}^{-1}\right]$ & $905^{\mathrm{a}}$ & - & 900 & 888 & 919,896 & 847 \\
\hline
\end{tabular}

${ }^{\text {a }}$ Spectra were not reproducible. $\frac{46}{}$ 

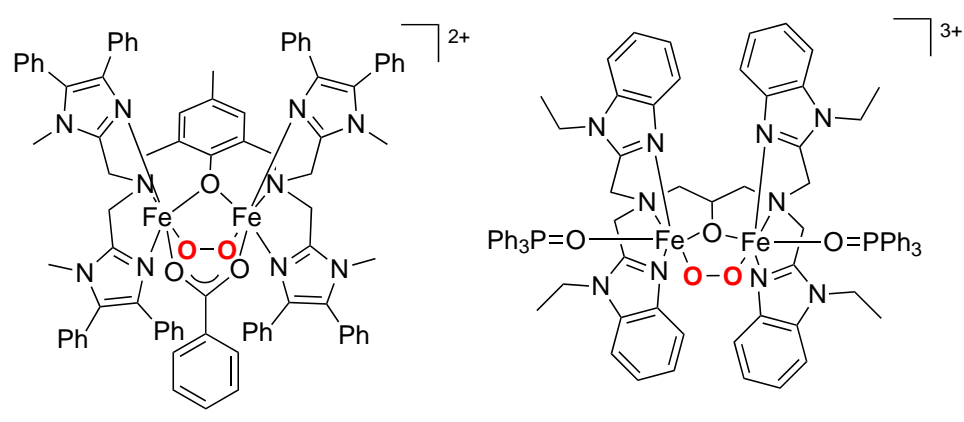

III
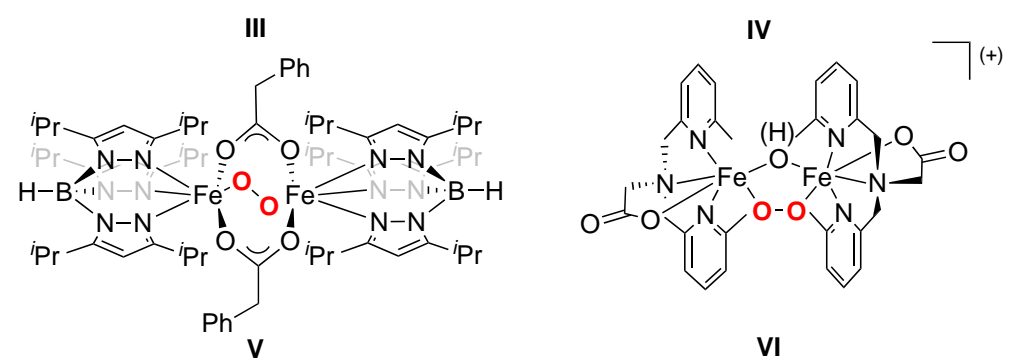

Scheme 1.10: Synthetic models for the peroxo-diiron core of the intermediate $\mathbf{P}$ of sMMO. The top two comlexes were obtained with dinucleating ligands, while the bottom representatives bear tridentate capping ligands.

\section{Models for the Intermediate Q}

Also the intermediate $\mathbf{Q}$ in sMMO has not been structurally characterized to date. Oxidation states of the diiron core as well as the coordination geometry of the $\mathrm{Fe}_{2} \mathrm{O}_{2}$ unit have been questioned for many years. Opinions have been varying between the existence of a closed core with $\mu$-oxo bridging ligands (diamond core) and open shell options with terminal oxo-ligands. 63] Even mixed, bridging and terminal, coordination modes were considered. However, recent Raman data obtained by LiPSCOMB and ProshlyAKOv suggest a closed diiron diamond core when compared to Raman signatures of different model complexes. 37 .

The first models for the intermediate $\mathbf{Q}$ of sMMO were published in the late 1990ies by the Que group. 64. They employed tris(pyridylmethyl)amine (tpa) capping ligands with various substituents to stabilize the diamond core motif. Although these systems in their $\mathrm{Fe}(\mathrm{IV}) \mathrm{Fe}(\mathrm{IV})$ form have not been structurally authenticated until today, extended spectroscopic details were provided in the late 2000s. [65] Also their mixed valent analogues and open core structures were investigated in presence and absence of protons and substrates. 66 68] The high valent Fe(IV)Fe(IV) diamond cores, however, exhibit a low spin state for each iron site whereas the spin state in the intermediate $\mathbf{Q}$ in sMMO bears two high spin ions. When opening the core upon reduction, the mixed valent $\mathrm{Fe}(\mathrm{IV}) \mathrm{Fe}(\mathrm{III})$ species is high spin and consequently displays a remarkably higher affinity toward substrate oxygenations. 67. Spectroscopic parameters of the diamond core stabilized with tpa ligands on the other side are closely related to those found for $\mathbf{Q}$ (Table 1.4). A small disadvantage in the use of tpa ligands can be seen in the instability of their diiron complexes in solution and during catalysis as they might dissociate in two mononuclear pendants. $\frac{69}{}$

A second ligand system that has received some attention in stabilizing high valent diiron 

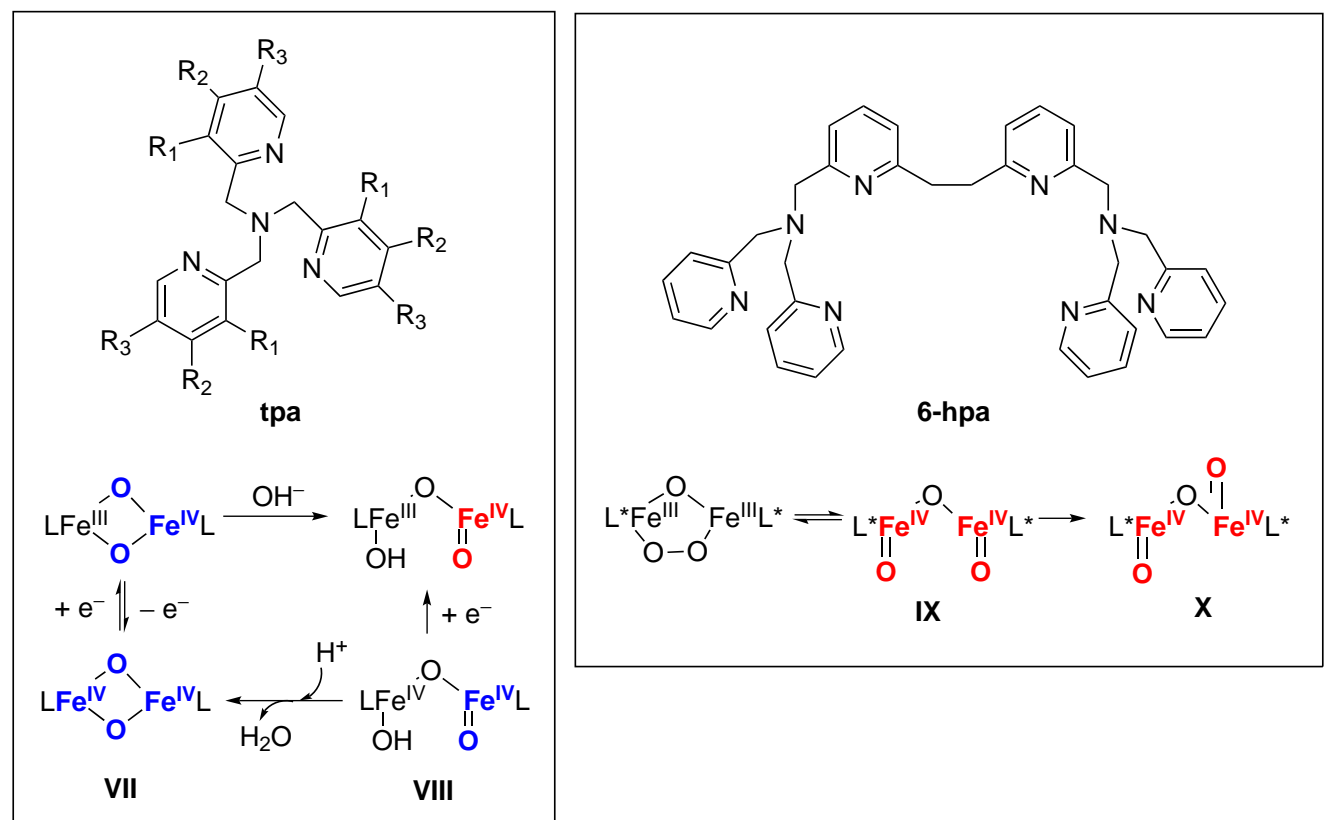

Scheme 1.11: High valent intermediates with the ligand systems tpa $[67 \mid 70$ and 6-hpa 70 . 72 . The residues $R_{1}$ to $R_{3}$ represent hydrogen atoms, alkyl- or alkoxy groups. High spin iron sites are highlighted in red whereas low spin sites are labeled in blue. High spin in this respect does refer to the local iron site, not the diiron core.

cores has been developed by KODERA and coworkers. The dinucleating ligand 6-hpa resembles a fusion of two tpa ligands connected by an ethylene linker to impart stability and avoid a cleavage of the diiron core. ${ }^{73}$ While tpa ligands are capable of stabilizing both, open and closed diamond core structures, the 6-hpa system seems to favor an open core with two terminal oxo ligands. Interestingly, the open diiron(IV) oxo core (IX) is metastable and exists in a temperature dependent equilibrium with the respective diiron(III)-peroxo species. ${ }^{71]}$ From Mössbauer and Raman studies a $S=2$ ground state was postulated for the high valent open diiron oxo core which agrees with findings for the intermediate $\mathbf{Q}$ in sMMO. According to the authors, the activation of substrates proceeds via a core rearrangement from a $s y n-\mathrm{Fe}=\mathrm{O}$ conformation to an anti form $(\mathbf{X})$. 72 .

Scheme 1.11 depicts the two described ligands and their spectroscopically observed diamond core structures. Table 1.4 lists relevant spectroscopic parameters for the described model systems and the intermediate $\mathbf{Q}$.

Table 1.4: Comparison of spectroscopic and structural parameters of the intermediate $\mathbf{Q}$ with respective model systems.

\begin{tabular}{|c|c|c|c|c|}
\hline & $\mathrm{Q}^{3 / 37 / 74 / 76}$ & VII 65 & VIII 66 & $\mathbf{I X} / \mathbf{X}^{71-73}$ \\
\hline$\lambda[\mathrm{nm}]$ & 330,430 & 485,875 & $705^{\mathrm{a}}$ & $500,610,783^{\mathrm{a}}$ \\
\hline$\epsilon\left[\mathrm{M}^{-1} \mathrm{~cm}^{-1}\right]$ & 7500,7500 & 9800,2200 & $2500^{\mathrm{a}}$ & $1092,821,204^{\mathrm{a}}$ \\
\hline$\delta\left[\mathrm{mm} \mathrm{s}^{-1}\right]$ & 0.17 & -0.04 & $-0.03,0.00$ & 0.13 \\
\hline$\left|\Delta E_{\mathrm{Q}}\right|\left[\mathrm{mms}^{-1}\right]$ & 0.53 & 2.09 & $0.92,1.96$ & 0.44 \\
\hline$\tilde{\nu}\left(\mathrm{Fe}^{\mathrm{IV}}-\mathrm{O}\right)\left[\mathrm{cm}^{-1}\right]$ & 690 & 674 & - & $821^{t}$ \\
\hline
\end{tabular}

${ }^{\mathrm{a}}$ In equilibrium with the peroxo species. 


\subsection{Nitric Oxide in Biological Systems}

The nitric oxide molecule (NO) is a radical found as a general product of the combustion of nitrogen containing substances in the air (reactive oxygen species) and among other functions serves as physiological messenger molecule in the human body. ${ }^{77}$ In its biological context nitric oxide can either be cytotoxic or cytoprotective. Cytoprotective ways involve signal transduction in both the peripheral and central nervous system, whereas cytotoxic effects might include its antagonizing effect against invading microorganisms, viruses, and neoplastic tissue. ${ }^{[77 / 78]}$ In addition, NO is a compulsory intermediate in the process of bacterial denitrification. ${ }^{[79}$ The nitric oxide radical is a diatomic molecule and bears an unpaired electron in one of its $\pi^{*}$ orbitals. Due to this radical character NO exhibits high reactivity and readily reacts upon oxidation and reduction to its conjugates $\mathrm{NO}^{+}$and $\mathrm{NO}^{-}$. 80. With dioxygen the formation of $\mathrm{NO}_{2}$ is favored, as is the formation of the conjugate acid $\mathrm{NO}^{-}$in aqueous environments under reductive conditions. 77 .

In metal complexes NO usually is found to bind via the nitrogen atom to a metal center. The metal-NO unit can adopt linear or bent geometries. In general bonding in metal nitrosyl complexes would be described as follows: The $\sigma$ orbital of the nitrogen atom forms a coordinative bond to the metal, which is able to backdonate electron density from its $\mathrm{d}_{\pi}$ orbital to the $\pi^{*}$ orbitals of NO (Scheme 1.12, 80.

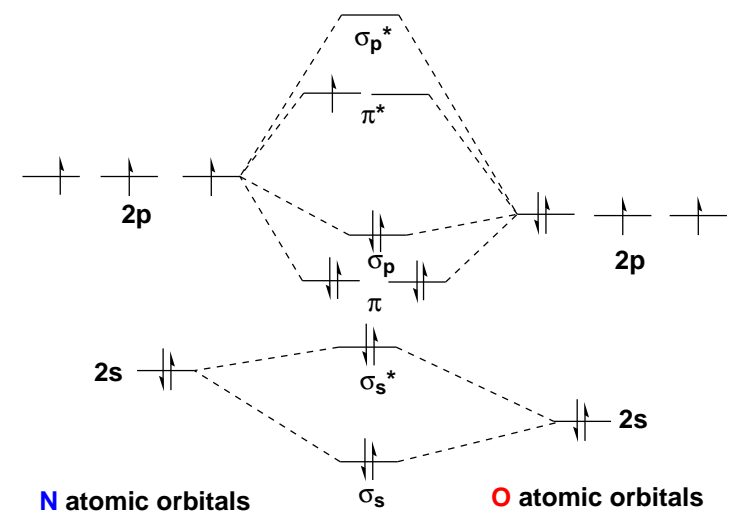

Scheme 1.12: Molecular orbital scheme of nitric oxide. 80 .

This imposes the effect that within the $\mathrm{M}-\mathrm{N}-\mathrm{O}$ unit the $\mathrm{M}-\mathrm{N}$ bond is rather strong, whereas the $\mathrm{N}-\mathrm{O}$ bond is relatively weak, compared to the less electronegative related ligand carbon monoxide. A Hückel study on a diiron dinitrosyl model complex $\left[\mathrm{Fe}_{2}(\mathrm{NO})_{2}\right.$ $\left.\left(\mathrm{NH}_{3}\right)_{6}\left(\mathrm{O}_{2} \mathrm{CH}\right)(\mathrm{OH})\right]^{2+}$ was performed by the LippaRD group to shed light on the bind-

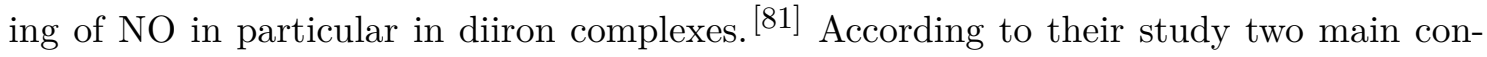
tributions influence the binding angle between metal and nitrosyl. For once the HOMO $\mathrm{Fed}_{\mathrm{xz}} / \mathrm{Np}_{\mathrm{x}}$ displays considerable $\pi$-antibonding character. Bending the nitrosyl would reduce this antibonding character. An ideal angle in this respect would be $120^{\circ}$. Contrary to this interaction $\pi$ bonding occurs via the Fe $d_{\mathrm{xz}}$ and the $\pi^{*}$ orbital of NO. In this respect the overlap of the two orbitals is increased by a more linear geometry. Overall the binding of NO to a metal differs in every ligand environment and cannot be generalized. Always an interplay of different interactions has to be considered. 
As the reduction of $\mathrm{NO}$ to the less toxic $\mathrm{N}_{2} \mathrm{O}$ is very relevant in nature, the knowledge of reduction potentials in this respect is significant. The reduction potential of free NO to $\mathrm{NO}^{-}$has been investigated by means of cyclic voltammetry. In a study from 2002 [77] the reduction of $\mathrm{NO}$ to ${ }^{3} \mathrm{NO}^{-}$has been calculated to lie at potentials of about $-0.8( \pm 0.2) \mathrm{V}$ vs NHE whereas the reduction to ${ }^{1} \mathrm{NO}^{-}$occurred at more negative potentials of about $-1.7( \pm 0.2) \mathrm{V}$. Experimentally the reduction of $\mathrm{NO}$ to ${ }^{3} \mathrm{NO}^{-}$in an aqueous solution at $\mathrm{pH}=7$ occurred at $-1.02 \mathrm{~V}$ and $-1.41 \mathrm{~V}$ respectively. This implies that the reduction is considerably $\mathrm{pH}$ dependent. In the presence of protons the reduction potential is shifted to more positive potentials.

\subsection{The Enemark-Feltham Notation}

To rationalize the highly covalent bonding in metal-nitrosyl complexes ENEMARK and FELTHAM introduced a separate notation to describe such a system. ${ }^{82}$ In this concept the number of $\pi^{*}$ electrons of the NO-ligand is added to the number of d-electrons of a metal. For a six-coordinate Fe(II) complex with six d-electrons of the metal ion, the NO. ligand and five neutral ligands, according to the notation a $\{\mathrm{Fe}(\mathrm{NO})\}^{7}$ species could be formulated. The Enemark-Feltham notation, however, avoids to assign localized charges and oxidation states due to the high covalency of the M-NO bond and since the orbital energies for $\mathrm{NO}^{+}, \mathrm{NO}$ and $\mathrm{NO}^{-}$are very similar. $\frac{80182}{}$ The previously described $\{\mathrm{Fe}(\mathrm{NO})\}^{7}$ complex could just as well originate from a $\mathrm{Fe}(\mathrm{III})$ species with five d-electrons and a $\mathrm{NO}^{-}$ ligand. For a definite assignment of the charge distribution structural and spectroscopic details are of key importance. Relevant parameters that can elucidate this aspect are the binding angle of the $\mathrm{M}-\mathrm{N}-\mathrm{O}$ unit and the infrared stretching frequencies of $\mathrm{NO}$ and $\mathrm{Fe}-\mathrm{N}$, as well as ${ }^{14 / 15} \mathrm{~N}-\mathrm{NMR}$ chemical shifts. ${ }^{80}$

\subsection{Nitric Oxide Reductases}

Similar to the iron oxygenases, also this class of enzymes can be subdivided into heme and non heme iron nitric oxide reductases. All of them contain dinuclear iron centers. $\frac{83}{3}$ Heme diiron nitric oxide reductases, or shortly termed NORs, feature a heme iron center termed heme- $\mathrm{b}_{3}$ located in close proximity to a non heme iron center $\mathrm{Fe}_{\mathrm{b}}$. $\frac{84}{\text {. This structural }}$ motif is largely found in denitrifying bacteria. NorBC, cNOR or heme $\mathrm{b}_{3}-\mathrm{Fe}_{\mathrm{B}}$ are among other enzymes associated with heme nitric oxide reductase activity and this recurring

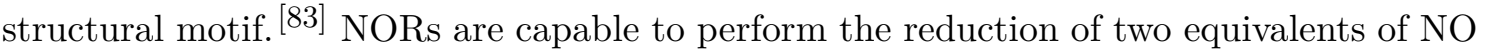
with two electrons and two protons to $\mathrm{N}_{2} \mathrm{O}$. The mechanism of this process is to date not fully elucidated. Synthetic models have been developed to perform binding studies of NO to the heme iron center and to study electronic effects that might influence NO reduction. 85 90] Apart from these heme/non heme NORs another family of enzymes has gained significant interest in literature. The large family of non heme diiron enzymes has been studied for decades with regard to oxygen activation. Today it is known that certain representatives, the Flavodiiron Proteins (FDPs), are especially suitable to reduce aside 
from dioxygen also nitric oxide. The following section will discuss this very intriguing group of enzymes in more detail.

\subsubsection{Flavodiiron Proteins (FDPs)}

Flavodiiron proteins, expressed by some microorganisms to fight the immune response in mammals, were developed to sense and detoxify nitric oxide. ${ }^{91}$ Apart from their function of reducing nitric oxide to the less toxic nitrous oxide they are as well able to activate dioxygen. Literature therefore attributes this class of enzymes a dual functionality. 9192 Generally though, FDPs are either more selective for nitric oxide (from E. coli and Salmonella enterica) or for dioxygen (e.g., eukaryotic FDPs from anaerobic protozoa or from methanogens). 91. Most FDPs contain a Flavin mononucleotide (FMN) cofactor and a non heme diiron site. The first flavodiiron protein was structurally characterized in 2000 from Desulfovibrio gigas as oxido-reductase. 93. The protein is a homodimer. Each monomer is composed of two domains, a $\beta$-lactamase unit with the non heme diiron center and a flavodoxin domain with the FMN cofactor. Within the non heme diiron site an oxygen molecule was located, which led to the conclusion that the discovered protein belonged to the family of diiron oxygenases. A second crystal structure from Moorella thermoacetica FprA (A-type flavoprotein) was published by LANZILOTTA and coworkers in 2005. 94. Three forms of the FDPs active site were reported: an oxidized form, a dithionite reduced and a dithionite form that had reacted with NO.

Different from the FDP from Desulfovibrio gigas, two homodimers were found in the structure. Each homodimer though bears, as decribed above, two domains in close proximity containing the FMN cofactor and the non heme diiron site. The diiron core is coordinated by in total four terminal histidines and three carboxylates from glutamate and aspartate. One bridging solvent molecule completes the coordination sphere. Scheme 1.13 depicts ligands and coordination modes of the diiron site in $\mathbf{F p r A}$. The arrow marks the only difference to the structure of the protein from Desulfovibrio gigas where one histidine is being exchanged for a water molecule. 95 .

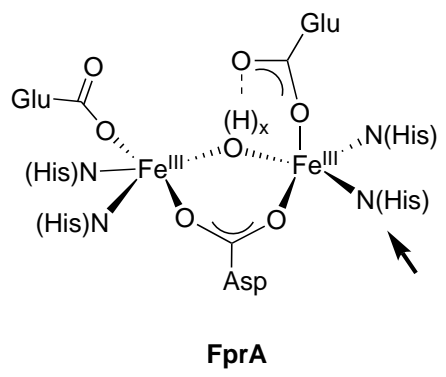

Scheme 1.13: Schematic representation of the diiron active site in Moorella thermoacetica.

The FMN cofactor is assumed to contribute to the fast electron transfer and thus FNOR reactivity of the enzyme. ${ }^{96}$ It is located in a proximity of 4- $6 \AA$ to the active site. Its exact function in the enzymes' catalytic cycle though is not known to date. 97. Not known yet is moreover the binding of nitric oxide to the diiron core. The crystallographic structure of FprA in 2005 was obtained of a diiron site without nitric oxide or dioxygen bound. Nitric oxide was modeled to fit in the diiron pocket via a space filling model. ${ }^{94}$. With this key 
aspect not elucidated, it is not surprising that the catalytic cycle and the process of nitric oxide reduction are under discussion in literature. Among many proposals basically four mechanisms are considered for the following reaction sequence:

$$
2 \mathrm{NO}+2 \mathrm{e}^{-}+2 \mathrm{H}^{+} \longrightarrow \mathrm{N}_{2} \mathrm{O}+\mathrm{H}_{2} \mathrm{O}
$$

An overview of the different mechanisms for the reduction of nitric oxide is given in Scheme 1.14

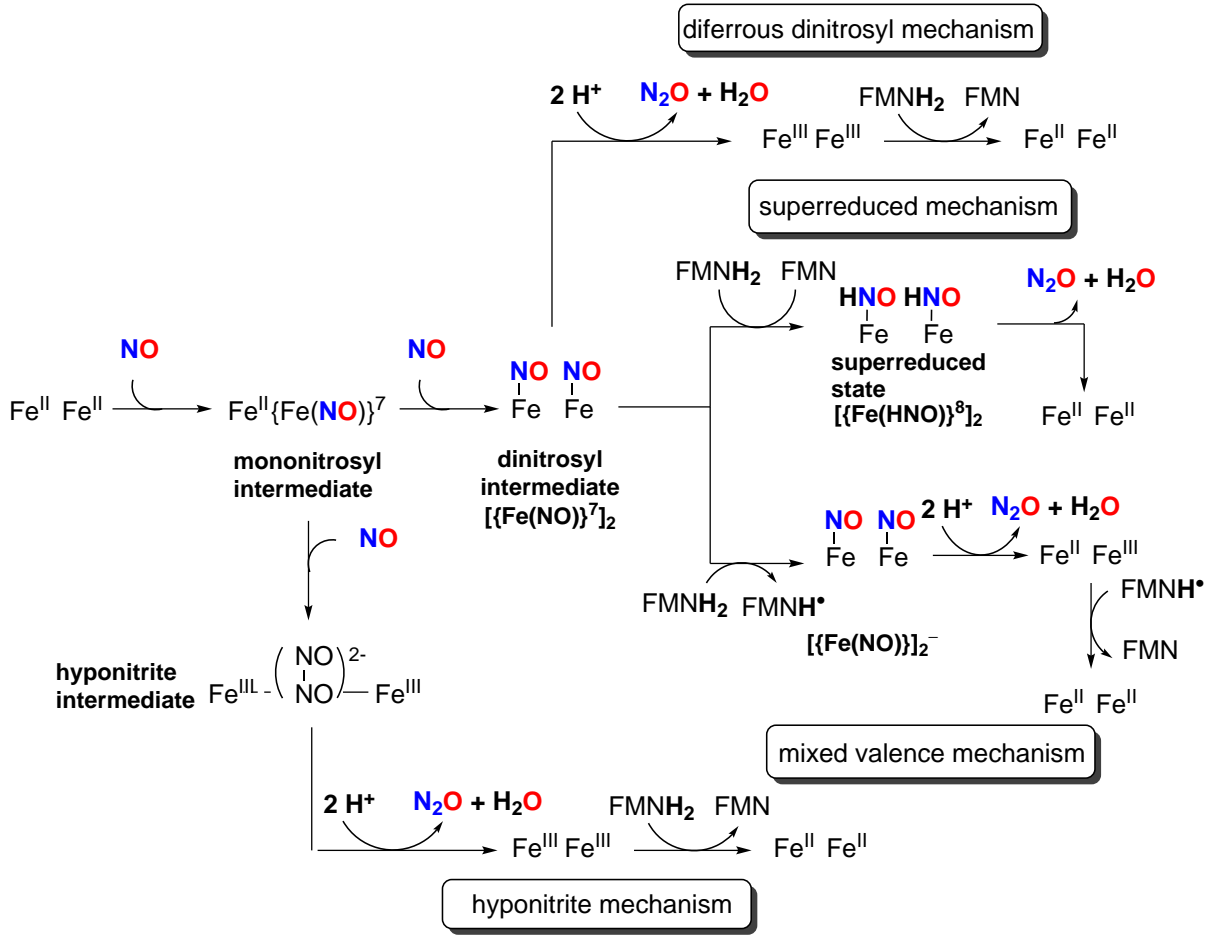

Scheme 1.14: Selection of proposed mechanisms for the reduction of $\mathrm{NO}$ and $\mathrm{N}_{2} \mathrm{O}$ generation by flavodiiron proteins. $96[98]$

In three of the four pathways a diferrous dinitrosyl intermediate is proposed. This intermediate can be reduced either directly by the addition of protons via the diferrous dinitrosyl pathway, by the sequential reduction mediated by the flavin mononucleotide cofactor and subsequent protonation via the mixed valence mechanism or by an $\mathrm{H}$-atom transfer through the cofactor via a superreduced mechanism. A fourth possibility lies in the formation of a hyponitrite intermediate followed by protonation and $\mathrm{N}_{2} \mathrm{O}$ release.

Recent studies reacting the dithionite reduced diiron center of FDPs with sequential equivalents of NO support the formation of a diiron dinitrosyl species, which would disregard the hyponitrite mechanism. 99] The formation of the dinitrosyl intermediate in this study seems to proceed via a mononitrosyl species first, which has been trapped by stopped flow $\mathrm{UV} / \mathrm{V}$ is measurements and EPR studies. The spin state of each iron center in the diiron dinitrosyl species was determined to $3 / 2$ with characteristic Mössbauer parameters. The diiron dinitrosyl sites were found to be antiferromagnetically coupled resulting in an $S=0$ ground state. Aside from the observation of these two species the reaction pathway of NO reduction in FDPs is still unknown. Calculations point toward a superreduced mech- 
anism [97. whereas experimentally and from model chemistry no option can be reliably ruled out as even the hyponitrite mechanism has been postulated for recent diruthenium dinitrosyl- and tricopper nitrosyl complexes. 100[101.

\subsection{Functional Diiron Models for NO-Reduction}

Mainly two groups have advanced model chemistry of diiron dinitrosyl complexes over the last couple of years. The very first model system of a $\left[\mathrm{Fe}(\mathrm{NO})^{7}\right]_{2}$ intermediate $(\mathbf{X I})$ was

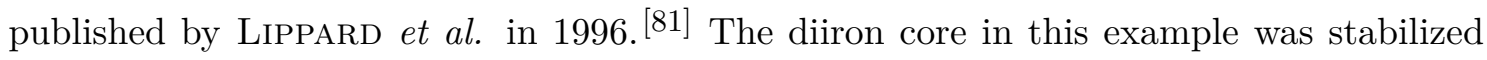
by a dinucleating ligand N-Et-hptb providing a $\left\{\mathrm{N}_{3} \mathrm{O}\right\}$ donorset for each iron atom. As coligand a carboxylate bridge was introduced. The two nitrosyl ligands coordinate in a syn fashion and are spatially quite close. Mössbauer data confirmed an $S=3 / 2$ spin state for each iron ion and the two metal ions were antiferromagnetically coupled. Upon oxygenation of the diiron dinitrosyl complex the formation of a corresponding diiron dinitrate species was observed. Further insights in the complex' behavior upon reduction were not provided until 2015. 102 Upon exposure to light at room temperature and at $15 \mathrm{~K}$, $\mathrm{N}_{2} \mathrm{O}$ formation was observed. Mechanistically the authors propose that upon irradiation a mononitrosyl intermediate is formed as they observed a new band in the infrared spectrum $\left(1695 \mathrm{~cm}^{-1}\right)$ compared to the signature of the initial dinitrosyl stretching frequency $\left(1784 \mathrm{~cm}^{-1}\right)$. In a subsequent step a putative hyponitrite species is possibly formed via an electrophilic attack of the second $\mathrm{NO}$ molecule, which finally yielded $\mathrm{N}_{2} \mathrm{O}$.

A second model (XII) that is capable of producing $\mathrm{N}_{2} \mathrm{O}$ upon reduction was presented by the LEHNERT group in 2013. 103. A similar dinucleating ligand system with $\left\{\mathrm{N}_{3} \mathrm{O}\right\}$ donor set was chosen. Instead of benzimidazole moieties, the ligand system contained pyridine side arms. Propionate or benzoate was employed as bridging coligand. ${ }^{83}[103] \mathrm{In}$ the diiron dinitrosyl complex both iron atoms were found to be high spin with $S=3 / 2$ and antiferromagnetically coupled. Upon chemical reduction of the propionate bridged version of these complexes with cobaltocene or $\mathrm{KC}_{8}$, complete conversion to $\mathrm{N}_{2} \mathrm{O}$ was observed. 103 As a mechanism the so called super reduced pathway has been suggested. This assumption was supported by electrochemical studies as the reduction of the Fe-NO sites occurred in a single $2 \mathrm{e}^{-}$transfer process and not sequentially.

Scheme 1.15 depicts the two described systems.

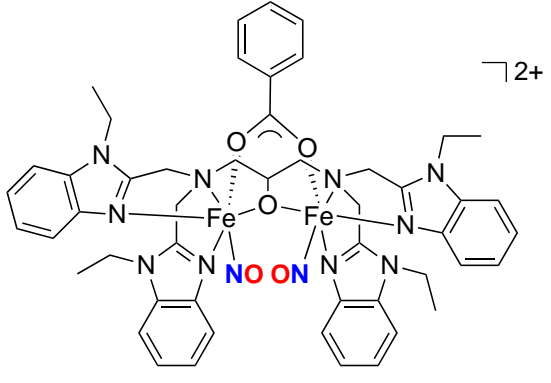

XI

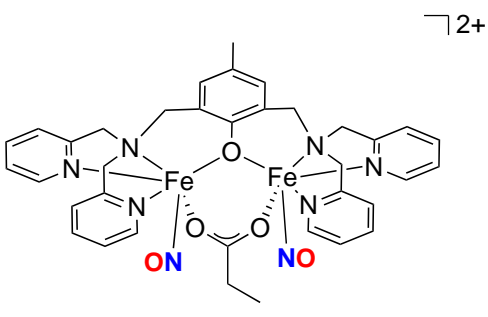

XII

Scheme 1.15: Diiron dinitrosyl model complexes for the reduction of $\mathrm{NO}$ to $\mathrm{N}_{2} \mathrm{O}$. 
A detailed comparison of their structural and spectroscopic signatures is given in Table 1.5 .

Table 1.5: Comparison of structural and spectroscopic parameters of models XI and XII with those of FprA.

\begin{tabular}{l|lll}
\hline & $\begin{array}{l}\text { FprA- } \\
\text { NO reacted }\end{array}$ & XI $\left.{ }^{\text {941 }}\right]$ & XII 103] \\
\hline $\mathrm{Fe} \cdots \mathrm{Fe}[\AA]$ & $3.23-3.57^{\mathrm{a}}$ & 3.44 & 3.47 \\
$\mathrm{~N}-\mathrm{O}[\AA]$ & $1.16^{\mathrm{a}}$ & 1.15 & $1.16,1.17$ \\
$\mathrm{Fe}-\mathrm{N}-\mathrm{O}\left[{ }^{\circ}\right]$ & $167^{\mathrm{a}}$ & 167 & 156,145 \\
$\mathrm{~N} \cdots \mathrm{N}(\mathrm{NO})[\AA]$ & $2.80^{\mathrm{a}}$ & 2.82 & 2.80 \\
$\lambda[\mathrm{nm}]$ & 453 & 520,620 & 410 \\
$\epsilon\left[\mathrm{M}^{-1} \mathrm{~cm}^{-1}\right]$ & 2000 & 395,579 & $\sim 2000$ \\
$\delta\left[\mathrm{mm} \mathrm{s}^{-1}\right]$ & 0.71 & 0.67 & - \\
$\left|\Delta E_{\mathrm{Q}}\right|\left[\mathrm{mm} \mathrm{s}^{-1}\right]$ & 1.85 & 1.44 & - \\
$\tilde{\nu}\left[\mathrm{cm}^{-1}\right]$ & - & 1785 & 1760 \\
\hline
\end{tabular}

${ }^{\mathrm{a} B o n d}$ distances and angles from calculations. 


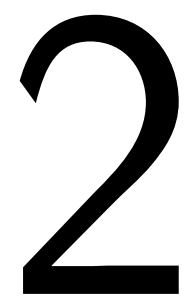

\section{Aim of this Work}

Low molecular weight analogues for enzymatic non heme diiron sites are important for understanding the function of these enzymes and in particular the potential mechanisms for the activation of small molecules. Efforts in this field have been made apart from elucidating biological functions to develop efficient catalysts for controlled reactions under mild conditions that would otherwise require immense energy resources.

The aim if this work is to develop new diiron complexes with monoanionic, pyrazolate bridged, bis(pentadentate), entirely nitrogen based chelating ligands to obtain functional models of non heme diiron sites for the activation of nitric oxide and dioxygen. The spectroscopic and structural characterization of precursors and intermediates during this process is pursued to obtain a deeper understanding of reaction pathways and catalytic events.

The anticipated diiron complexes with nitrogen based bis(pentadentate) ligand scaffolds differ from the active sites of biological of non heme diiron proteins. However, it is known that nitrogen donors impart stability while carboxylates are prone to dissociate or feature variable coordination modes. Moreover, high valent intermediates in diiron oxo model chemistry have been realized until today with largely nitrogen based ligands.

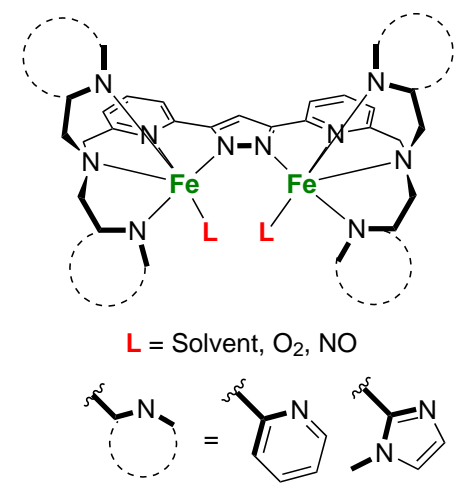

Scheme 2.1: Targeted pyrazolate based diiron complexes with nitrogen based amine side arms.

In the last couple of years new pyrazolate/pyridine based scaffolds have been developed which have been shown to bridge two metal sites via the nitrogen atoms of the pyrazo- 
late. 104]105] By the introduction of amine based side arms with additional pyridine or imidazolyl moieties, chelating nitrogen based ligands that should incorporate two iron centers can be obtained. The new diiron complexes should bear at least one vacant coordination site to bind labile exogenous coligands such as solvent molecules and allow for an access and binding of small molecules and/or substrates.

The diferrous form of these new molecules should serve as a platform for new reactivity and thus should be fully understood spectroscopically, structurally and electronically.

Consequently, these new models should also be investigated towards their ability to bind small molecules, in particular dioxygen and nitric oxide. Provided that this reactivity is accessible with these novel diiron complexes, a thorough analysis is anticipated to gain insight into their redox behavior and the catalytical transformation of these small molecules. Ultimately, their reactivity with respect to substrate activation and applicability should be tested. 


\section{Design and Synthesis of Pyrazolate based Bis(pentadentate) Ligand Scaffolds}

\subsection{Inspiration}

Ligand scaffolds that can host two metal sites are widely established in nature and model chemistry. The simplest ligands that can bridge two metal sites are single atom bridges such as halides, oxides or sulfides (e.g. as shown in the previously described diamond core structures). 106. Di- and triatomic bridging units as, e.g., pyrazolates or carboxylates can be used to tune the metal-.metal distance, which has an influence on many factors such as reactivity, cooperativity and stability. Pyrazolates, however, outrival simple single atom bridges as they can be functionalized in the 3, 4, and 5-position to introduce additional donor sites that can stabilize the metal core, contribute to the overall chelation of the metal centers and thus enhance the chelate effect. 107-110 Pyrazoles in their deprotonated form serve as monoanionic ligands and are therefore beneficial to stabilize metals in higher oxidation states.

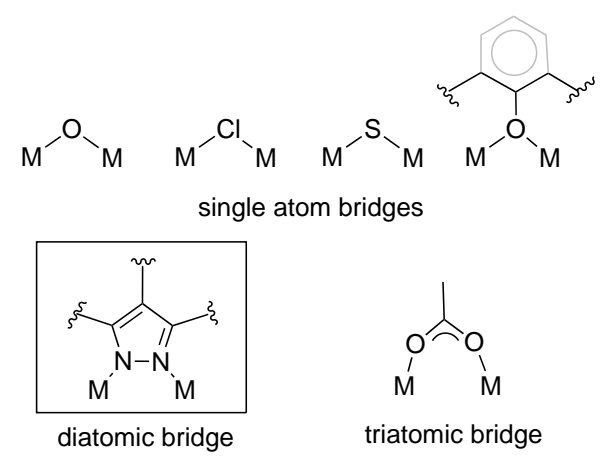

Scheme 3.1: Examples for single atom, diatomic (pyrazolate) and triatomic bridging ligands.

Moreover, small nitrogen containing heterocycles are close mimics for nitrogen donor sites in non-heme diiron proteins such as the imidazole group of the amino acid histidine. 111. A clear advantage for the use of a pyrazolate over a carboxylate as bidentate bridging ligand in model chemistry lies in the lability and flexibility of carboxylate binding modes known as the carboxylate shift or twist. 71112 114] The carboxylate shift may be beneficial in enzymatic oxygenation reactions and substrate uptake, in model chemistry, however, the binding mode of carboxylates is hardly controllable. 
In the MEYER group, pyrazolate based compartmental ligands were established for a series of polynuclear metal complexes. 115]117. Pyrazoles functionalized in the 3- and 5-position are covalently connected with a huge library of side arms. The design of a ligand for a certain bimetallic site involves a couple of considerations. The ligand framework can be altered at basically two positions. One is the variation of the spacer between the two metals, which would result in the modification of the pyrazole building unit. Another option that may alter the denticity is the choice of a specific side arm.

The bis(pentadentate) ligand scaffolds used in this work are inspired by a series of preceding complexes. Stable, oxidatively rugged ligands connecting two metal sites cooperatively via a bridging pyrazolate were developed for the application in $\mathrm{Ru}$-based water oxidation catalysis [118 121] (XIII, Scheme 3.2) and even before for the self assembly of [2×2]-Fe grid complexes (XIV, Scheme 3.2. 122, 124.
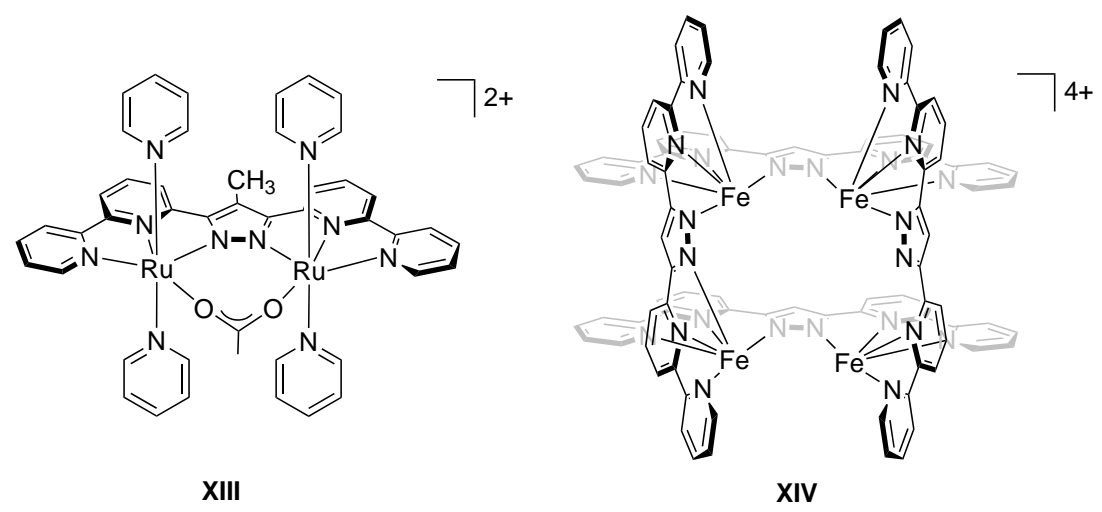

Scheme 3.2: Water oxidation catalyst XIII and $[2 \times 2]$ grid complex XIV with oxidatively rugged pyrazolate based ligand backbones.

A robust ligand design can be obtained by connecting the pyrazole in the 3 - and 5-position with small aromatic heterocycles such as pyridines. This is advantageous to prevent ligand degradation if metals in high oxidation states under rather harsh reaction conditions need to be stabilized. 125 For the activation of small molecules at diiron sites with subsequent catalytic events, high valent intermediates might have to be stabilized, in which case a robust ligand backbone might be beneficial.

Dinuclear iron complexes favor the coordination by five or six donor atoms for each metal site. Hence to design a chelating ligand that can fulfill this requirement, aside from the pyrazolate backbone additional side arms need to be introduced.

Ligands with tetradentate chelating moieties for dinuclear pyrazolate based iron complexes 1131126 128 and with pentadentate moieties for dinuclear pyrazolate based ruthenium complexes [120] have been presented before (Scheme 3.3. The systems shown in Scheme 3.3 in particular comprise ligand scaffolds with amine side arms bearing small $N$-donating heterocycles for the stabilization of the diiron core.

The bis(pentadentate) pyrazolate based ligand XVI previously used for bimetallic ruthenium complexes will be employed in this work for the synthesis of diiron complexes. 


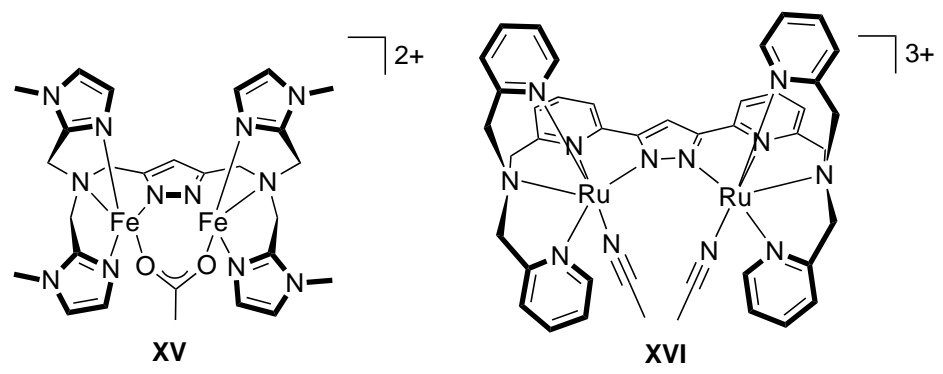

Scheme 3.3: Left: Pyrazolate bridged diiron complex with bis((methylimidazolyl)methyl)amine side arms for the activation of small molecules. Right: Ruthenium based bimetallic complex with picolylamine side arms.

\subsection{Anticipated Ligand Design}

The targeted ligands in this work are entirely nitrogen based. Iron(II) and iron(III) sites often prefer octahedral geometry with sixfold coordination. This geometry is anticipated to be realized by a bis(pentadentate) ligand scaffold with one vacant site for the coordination of a small molecule at each iron atom. The bis(pentadentate) ligand scaffolds are a fusion of bis(pyridyl)pyrazolates with bis((methylimidazolyl)methyl)amine and dipicolylamine side arms. Hence they are expected to profit from a robust ligand backbone with an additional donor site provided by the pyridine ring and the chelating strength of an overall bis(pentadentate) binding unit. By employing bis(pentadentate) entities the use of coligands such as carboxylates to stabilize the diiron core motif can be potentially avoided. Through the rigid ligand backbone the metal $\cdots$ metal separation is expected to be larger compared to ligands with more flexible methylene and/or ethylene units as in XV (Scheme 3.3). The distance, however, should still be appropriate to bind and activate small substrates and to mediate cooperativity between the two metal sites.

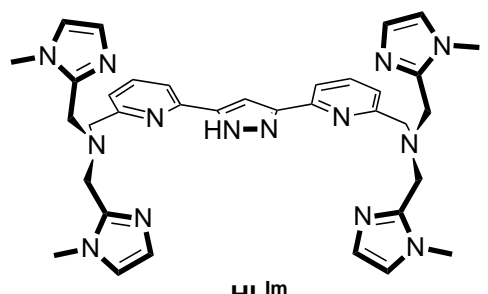

$H L^{I m}$

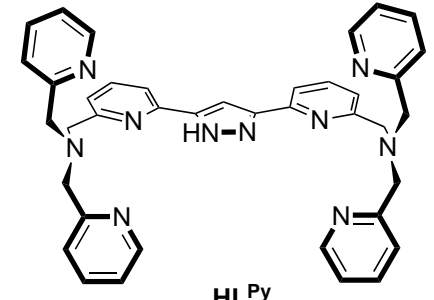

$\mathrm{HL}^{\mathrm{Py}}$

Scheme 3.4: Anticipated bis(pentadentate) ligand scaffolds with bis((methylimidazolyl)methyl)amine $\left(\mathbf{H L}^{\mathbf{I m}}\right)$ and dipicolylamine side $\operatorname{arms}\left(\mathbf{H L}^{\mathbf{P y}}\right)$.

\subsection{Synthetic Strategy}

\subsubsection{Synthesis of the Pyrazole Building Unit}

In 2007 Licheng Sun et al. published a new synthesis route for a pyrazolate building unit with pyridines connected to the 3 - and 5-positions of the pyrazolate. 104] Of particular interest in this approach is the functionalization of the pyridines in the 6-position. An optimized synthesis was developed by FisCHER in the framework of a PhD thesis. $\frac{105}{\square}$ 

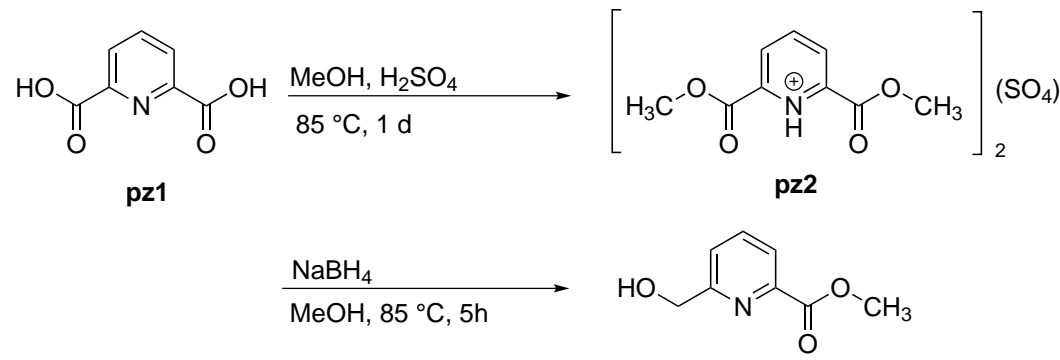

pz3

1.) NaOEt, EtOAc, toluene

$110{ }^{\circ} \mathrm{C}, 5 \mathrm{~h}$

2.) $\mathrm{H}_{2} \mathrm{SO}_{4}(20 \%)$

$100{ }^{\circ} \mathrm{C}, 3 \mathrm{~h}$

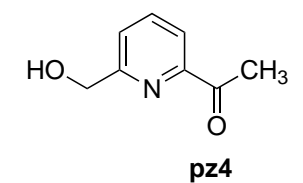

管

pz3<smiles>CC(=O)c1cccc(CO)n1</smiles>

pz4

1.) $\mathrm{NaOEt}$,

toluene, $50^{\circ} \mathrm{C}$

2.) $\mathrm{CH}_{3} \mathrm{COOH}$,

$\mathrm{H}_{2} \mathrm{O} ; 0^{\circ} \mathrm{C}$

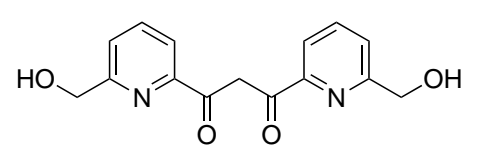

pz5
$\underset{\mathrm{EtOH}, 95^{\circ} \mathrm{C}, 4 \mathrm{~h}}{\stackrel{\mathrm{N}_{2} \mathrm{H}_{4} \cdot \mathrm{H}_{2} \mathrm{O}}{\longrightarrow}}$

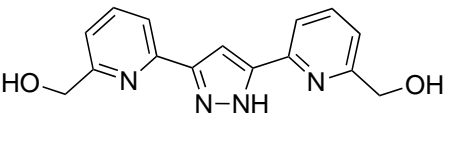

pz6

Scheme 3.5: Synthesis route by SUN and Fischer for a new bispyridylpyrazolate building block. Some of the original steps were modified for reasons of upscaling and optimization. 104 105.

Starting from 2,6-pyridine dicarboxylic acid, the treatment with sulfuric acid in methanol leads to an esterification of the carboxylates (pz2). In a following step these ester functions are partially reduced to yield an alcohol function on one side of the pyridine (pz3). Crucial in this reduction is the choice of equivalents of the reductant sodium borohydride. An access of reductant leads to a reduction of both ester functions as a side product. Also the grade of the reducing agent and the grain size of the hydride were found to be decisive for the overall yield of the reaction. Most reliable is the use of hydride granules instead of a powder, as they react more slowly with the substrate.

Half of the obtained product (pz3) was reacted with freshly distilled ethyl acetate. In the presence of sodium ethoxide as a base the $\mathrm{C} 2$ position of ethyl acetate is deprotonated yielding the enol form. The enolate can act as a nucleophile and undergo a nucleophilic attack at the ester function of the pyridine. A diketone is formed. Essential in this step of the synthesis is the use of dry solvents and freshly prepared sodium ethoxide. Sodium ethoxide should not be stored under air as it degrades over time. The remaining ester function of the diketone intermediate is hydrolyzed with sulfuric acid yielding a carboxylic acid functionality. A subsequent decarboxylation yields pz4.

With the obtained pyridine ester and ketone (pz3 and pz4) in the presence of the base 
sodium ethoxide and subsequent addition of acetic acid a new diketone is formed (pz5), which can be isolated as it cannot undergo decarboxylation. In a last step the 1,3-diketone (pz5) is reacted with hydrazine monohydrate in ethanol to yield the pyrazole building unit pz6. It should be noted that despite all of the individual steps were optimized in purity and yield, the overall yield is not very high. To obtain a reasonable amount of the pyrazole building block (5-10 g), one should begin with at least $100 \mathrm{~g}$ of the starting material.

The two alcohol functions of the pyrazole derivative pz6 were further functionalized to enhance their readiness for substitution and to facilitate side arm attachment. If stirred in thionyl-chloride for $1 \mathrm{~d}$ at ambient temperature and with an appropriate workup, the hydroxide can be exchanged for a chloride. By heating the pyrazole pz6 to reflux in hydrobromic acid and concentrated sulfuric acid an analogous exchange of the hydroxo functionality for a bromo function can be observed. Bromide and chloride allow for more facile $S_{N} 2$ substitution reaction conditions und thus for a better coupling of the side arms with the pyrazole unit. If required, the iodide analogue of the pyrazole building unit can be obtained by a simple Finkelstein reaction starting from pz7 (Scheme 3.6) with potassium iodide in acetone. This reaction is straightforward and proceeds in quantitative yield.

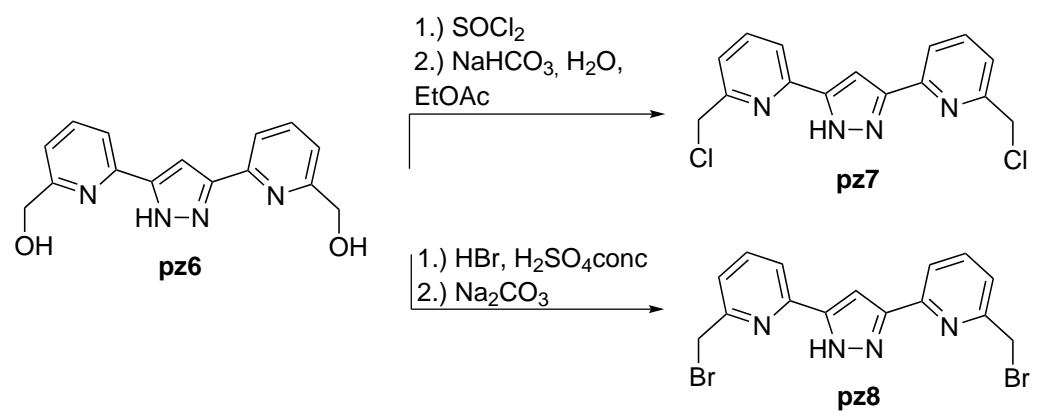

Scheme 3.6: Substitution of the OH-groups by chloride and bromide to give pz7 and pz8, respectively, following a route developed by FiscHER. 105

\subsubsection{Side Arms}

Two different side arms were used to synthesize the targeted ligand scaffolds. Bis((methylimidazolyl)methyl)amine $\left(\mathbf{s a}^{\mathbf{I m}}\right)$ can be prepared according to a literature procedure by OBERhausenet al.. 129$]$ Within the MEYER group the synthesis has been optimized various times and the amine can be prepared on a reasonable large scale and is readily available. $126\left[130\right.$ Dipicolylamine $\left(\mathbf{s a}^{\mathbf{P y}}\right)$ is commercially available or can be synthesized via a two step protocol according to literature from 2-aminomethylpyridine and 2pyridinecarboxaldehyde. 131 . For the synthesis of the targeted ligands commercially available dipicolylamine was used.

\subsubsection{Synthesis of $\mathrm{HL}^{\operatorname{Im}}$}

The synthesis of $\mathbf{H L}^{\mathbf{I m}}$ has first been developed in the framework of a preceding master thesis. $\frac{132}{132}$ In general, the pyrazolate pz7 is reacted with the bis((methylimidazolyl)methyl)amine side arm $\left(\mathbf{s a}^{\mathbf{I m}}\right)$ in acetonitrile solution. The addition of sodium carbonate 
buffers evolving hydrochloric acid. A screening of many solvents and solvent mixtures has revealed that dry acetonitrile is to date the most beneficial solvent for a successful conversion of the pyrazole building block and side arm. However, the workup of $\mathbf{H L}^{\mathbf{I m}}$ is very demanding. The crude product always contains residues of the free side arm. Separation of the final ligand and the side arm is challenging as they have similar solubility in almost all solvents. Also column chromatography is not sufficient for separation. The highest purity was obtained when stirring the crude product in water. The side arm shows a significantly higher solubility in water than $\mathbf{H L}^{\mathbf{I m}}$ which is advantageous for separation. The final ligand, however, is not entirely insoluble in water and becomes an oil due to its hygroscopic behavior. Several filtration steps yield the final product as a yellow oil. By coevaporation of this product with dichloromethane a yellow solid can be obtained. Due to many washings and purification steps, rather low yields between 10 and $20 \%$ had to be accepted.

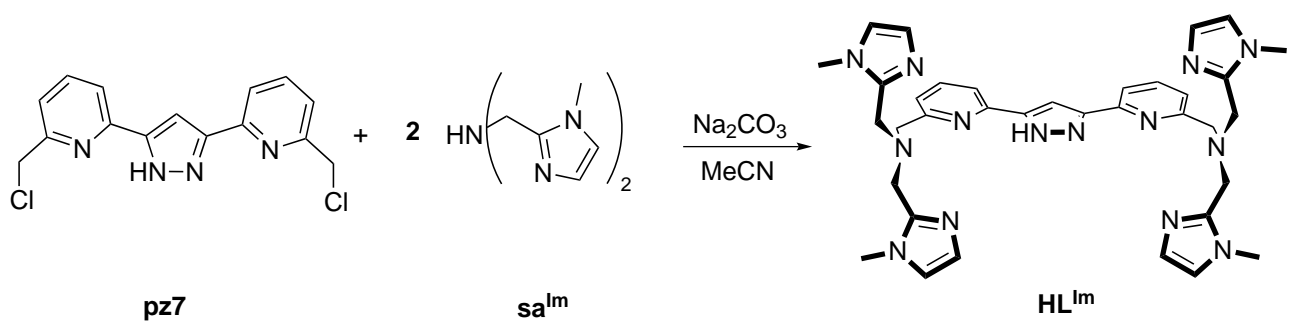

Scheme 3.7: Synthesis route for $\mathbf{H L}^{\mathrm{Im}}$.

\subsubsection{Synthesis of $\mathrm{HL}^{\mathrm{Py}}$}

For the synthesis of $\mathbf{H L}^{\mathbf{P y}}$ a route was followed first developed by SANDER. 120] The pyrazole building block pz8 and the dipicolylamine side arm $\left(\mathbf{s a}^{\mathbf{P y}}\right)$ were coupled in a $\mathrm{MeOH} / \mathrm{THF}$ 1:1 solvent mixture in the presence of sodium carbonate. The synthesis is straightforward and yields a crude brown product. After several workup steps the ligand can be obtained in good purity and yield as a yellow solid. The workup was slightly modified from the original procedure by including column chromatography as purification method in order to eliminate salt residues that might interfere with complex formation. The final ligand can be obtained as the hydrobromide salt.
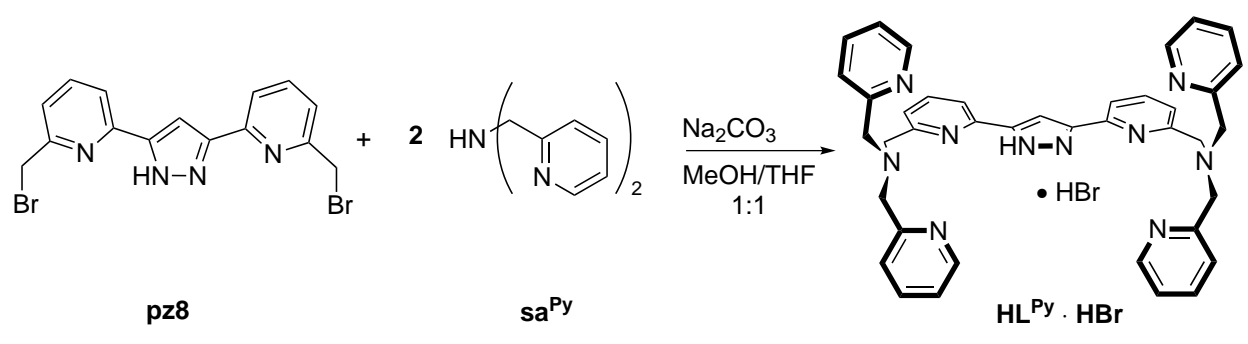

Scheme 3.8: Synthesis route for $\mathbf{H L}^{\mathrm{Py}}$. 


\subsubsection{Excursus: The Ligand $\mathrm{K}_{5} \mathrm{~L}^{\mathrm{COO}}$}

The ligand $\mathbf{K}_{5} \mathbf{L} \mathbf{C O O}$ was not synthesized in the course of this work but was provided by S. Fischer. As it was used for complexation reactions it should nevertheless be mentioned in this context. The ligand can be obtained by reacting the above described pyrazole unit pz8 with an amine side arm containing two ester functionalities. Subsequent alkaline saponification leads to the ligand $\mathbf{K}_{5} \mathbf{L}^{\mathbf{C O O}} .105[133$

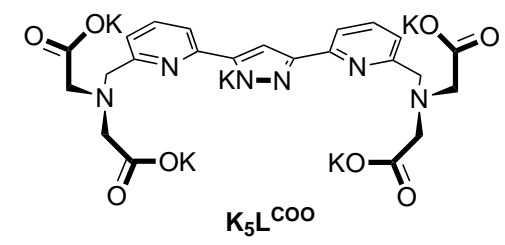

Scheme 3.9: The ligand system $\mathbf{K}_{5} \mathbf{L}^{\mathbf{C O O}}$ developed by Fischer. 105[133.

The ligand scaffold $\mathbf{K}_{5} \mathbf{L}^{\mathbf{C O O}}$ has not been employed to host two metal sites to date.

For the synthesis of dinuclear iron complexes, this ligand is very promising since it is soluble in water. Water soluble diiron complexes are of great interest for the development of homogeneous dinuclear water oxidation catalysts.

\subsection{Summary}

The exact emulation of the active sites of non-heme diiron complexes is very difficult. Reactivity, however, can be investigated with low molecular weight analogues for which the herein presented ligand scaffolds $\mathbf{H L}^{\mathbf{I m}}$ and $\mathbf{H L}^{\mathbf{P y}}$ provide a robust ligand design. The idea to incorporate dinuclear iron sites into the ligand scaffolds of $\mathbf{H L}^{\mathbf{I m}}$ and $\mathbf{H L}^{\mathbf{P y}}$ is new and has not been targeted to date. The synthesis of both ligands was optimized to a level of purity which was considered sufficient for successful complexation with iron salts.

A major target of their diferrous complexes is to study the activation of small molecules and reactivity by a variety of spectroscopic tools. The choice of side arms allows for comparison of different small $N$-heterocylic nitrogen donors, which can affect a variety of properties such as the electronic structure, spectroscopy and magnetism.

The ligand $\mathbf{K}_{5} \mathbf{L}^{\mathbf{C O O}}$ is considered as promising platform for attempts to perform water oxidation with dinuclear iron complexes due to its solubility in water and strong anionic character. 



\section{Synthesis and Characterization of Diferrous Complexes with Nitrile Coligands}

\subsection{Introduction}

Pyrazolate bridged iron complexes have been intensely studied by MEYER and coworkers with a focus on magnetochemistry in [2 x 2] grid complexes 122 124134], hysteretic bistability in biomimetic carboxylate bridged complexes [113|126|127] and lately spin crossover (SCO) in solution by ligand exchange reactions. 135 .

When incorporating $\mathrm{Fe}(\mathrm{II})$ sites into the previously described bis(pentadentate) ligand scaffolds, the obtained diiron complexes might exhibit interesting electronic and magnetic properties. In the targeted diiron(II) complexes, each Fe(II) center can adopt two possible spin states: high spin $(S=2)$ and low spin $(S=0)$. SCO requires a molecular spin state switching from a low spin to a high spin configuration and vice versa. A variation of the electron configuration in the $3 \mathrm{~d}$ orbitals of the iron ions is commonly associated with a change of metal-ligand bond lengths (Figure 4.1), resulting in changes of spectroscopic characteristics such as UV/Vis absorptions, the magnetic moment and Mössbauer parameters. 136137] SCO can be triggered by external stimuli like temperature, pressure, light or an applied magnetic field. $138 \mid 139$.

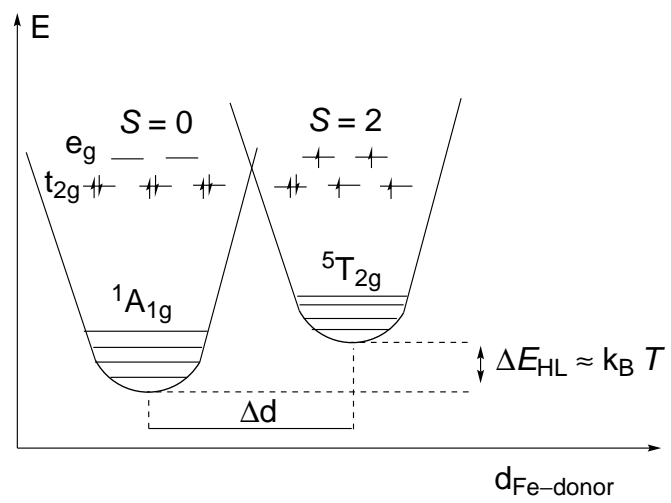

Figure 4.1: Schematic representation of the SCO relevant potential curves for a $\mathrm{d}^{6}$ ion (Fe(II)) with octahedral geometry.

Many examples in literature have shown that the energetic difference between high and low spin state strongly depends on the coordination environment of the metal ion. Furthermore 
the donor abilities of the ligand play a crucial role for the most energetically favorable spin state.

With respect to the spectrochemical series strong $N$-donating ligands are known to stabilize both states depending on the individual donor strength. [140] Thus a prediction of the spin state of the metal is challenging.

In this chapter the synthesis and characterization of diferrous complexes obtained with the novel pyrazolate based ligands $\mathbf{H L}^{\mathbf{I m}}$ and $\mathbf{H L}^{\mathbf{P y}}$ will be discussed. Besides their suitability for substrate activation their electronic and spectroscopic properties will be examined closely as they are important factors for reactivity.

\subsection{Synthesis and Structural Characterization of Diferrous Complexes with $\mathrm{HL}^{\mathrm{Im}}$}

In this section diiron complexes obtained with the ligand system $\mathbf{H L}^{\mathbf{I m}}$ are discussed. As coligands weakly coordinating solvents stabilize the diiron core but impart lability for subsequent small molecule activation. Different iron salts were selected for complexation and comparison of metric parameters. Moreover the anion influence on structural and spectroscopic features in the solid state is investigated.

\section{$\left[\mathrm{L}^{\operatorname{lm}} \mathrm{Fe}\{\mathrm{Fe}(\mathrm{MeCN})\}\right](\mathrm{OTf})_{3}, \mathrm{MeCN}(1 \mathrm{a})$}

\section{Synthesis and Crystallographic Structure}

Complex 1a can be obtained by reacting $\mathbf{H L}^{\mathbf{I m}}$ in dry acetonitrile with 2 eq. of $\mathrm{Fe}(\mathrm{OTf})_{2}$. $2 \mathrm{MeCN}$ in the presence of 1 eq. of $\mathrm{NaO} t \mathrm{Bu}$ as a base. Complex 1a crystallizes by diethyl ether diffusion into the reaction mixture in almost quantitative yield. Single crystals of $\mathbf{1 a}$ feature a dark red color. The compound crystallizes in the monoclinic space group $P 2_{1} / \mathrm{n}$ with one cation and three triflate anions in the asymmetric unit. One of the triflate anions was found to be disordered over two positions in a $0.71 / 0.29$ ratio. The bridging pyrazolate and the two iron atoms lie almost perfectly in a plane. Each iron atom is coordinated by the ligand in a pentadentate fashion. A striking feature of the structure is that only one iron atom bears an additional acetonitrile coligand. The overall coordination for this iron atom is pseudo-octahedral, whereas for the other atom a distorted square pyramidal coordination sphere with a $\tau_{\mathrm{s}}=0.42$ parameter 1 is observed. The Fe...Fe distance is $4.22 \AA$. A representation of the molecular structure of the cation of $\mathbf{1 a}$ is given in Figure 4.2 . An interesting structural parameter which will be relevant in the discussion of spin states is the angle $\mathrm{Fe}-\mathrm{N}-\mathrm{C}$ involving the coordinating acetonitrile solvent molecule. In 1a the angle is close to linearity around $175^{\circ}$. From orbital considerations, the nitrogen atom of the nitrile is sp-hybridized. The electrons which are provided for coordination to the metal occupy a sp-orbital. $\pi$-backbonding occurs from a d-orbital of the metal to the $\pi^{*}$ orbital of the nitrile. Thus a preference for a linear arrangement for the $\mathrm{Fe}-\mathrm{N}-\mathrm{C}$ unit can

\footnotetext{
${ }^{1}$ According to ADDISON and RAO the parameter $\tau_{\mathrm{s}}$ defines the degree of trigonality in five coordinate structures by the following expression: $\tau_{\mathrm{s}}=(\beta-\alpha) / 60$. 141. While for ideally trigonal bipyramidal structures a $\tau_{\mathrm{s}}$ value of 1 is expected, the value is close to zero for square pyramidal geometries.
} 


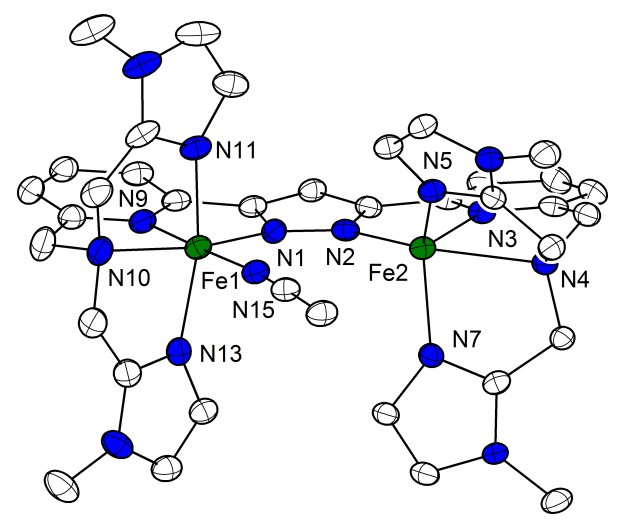

Figure 4.2: Crystal structure of the cation of $\left[\mathrm{L}^{\operatorname{Im}} \mathrm{Fe}\{\mathrm{Fe}(\mathrm{MeCN})\}\right](\mathrm{OTf})_{3}$, MeCN 1a. Thermal ellipsoids are set to $30 \%$. Hydrogen atoms, solvent molecules as well as the triflate anions were omitted for clarity. Selected bond lengths $[\AA]$ and angles $\left[^{\circ}\right]$ Fe1-N1 1.948(6), Fe1-N9 1.901(5), Fe1N10 2.066(6), Fe1-N11 1.955(6), Fe1-N13 1.996(6), Fe1-N15 1.938(6), Fe2-N2 2.071(5), Fe2-N3 2.109(5), Fe2-N4 2.385(5), Fe2-N5 2.052(5), Fe2-N7 2.053(5), Fe1-N15-C36 174.8(6), Fe1-N1N2-Fe2 2.151(3).

be expected. The pyrazolate acts as monoanionic ligand. With three triflate counterions the resulting oxidation state of both iron atoms in 1a is +II.

The comparison of bond lengths, however, reveals shorter $\mathrm{Fe}-\mathrm{N}$ distances for the sixcoordinate iron atom by approximately $0.1 \AA$. This gives rise to the assumption that the spin states of the ferrous ions Fe1 and Fe2 differ. The shorter distances indicate a low spin state for Fe1 while the spin state of Fe2 presumably is high spin. This spin state change from $\mathrm{Fe} 2$ to $\mathrm{Fe} 1$ is induced by the close to linear coordination of a sixth strong nitrogen donor provided by the nitrile.

\section{$\left[L^{\operatorname{Im}} \mathrm{Fe}\{\mathrm{Fe}(\mathrm{MeCN})\}\right]\left(\mathrm{BF}_{4}\right)_{3}(\mathbf{1 b})$}

Synthesis and Crystallographic Structure

Complex $\mathbf{1 b}$ can be prepared by an analogous synthesis route as noted for 1a. As iron salt the acetonitrile adduct $\left[\mathrm{Fe}(\mathrm{MeCN})_{6}\right]\left(\mathrm{BF}_{4}\right)_{2}$ was freshly prepared by Soxhlet extraction over molecular sieves from the commercially available aqueous salt. ${ }^{142}$ The complexation reaction was conducted in acetonitrile in the presence of 1 eq. $\mathrm{NaO} t \mathrm{Bu}$ as a base. Dark red single crystals were obtained by slow vapor diffusion of diethyl ether into the reaction mixture. $\mathbf{1 b}$ crystallizes in the triclinic space group $P \overline{1}$ with one threefold positively charged complex cation and three tetrafluoroborate anions in the unit cell. One of the $\mathrm{BF}_{4}^{-}$ counterions was found to be disordered over two positions in a $0.67 / 0.33$ ratio. Moreover the quality of the obtained crystallographic data was not very high. Since residual electron density probably belonging to cocrystallized solvent molecules could not be fully assigned, the SQUEEZE routine by the PLATON program was used to treat respective regions. Thus it is unclear if the unit cell bears additional solvent molecules that were not found due to the moderate quality of the structure. As already described for $\mathbf{1 a}$ the two iron centers are not equal but differ in the coordination of an acetonitrile ligand to only Fe1. Thus one iron ion is pseudooctahedrally coordinated and the other in a distorted trigonal bipyramidal fashion $\left(\tau_{\mathrm{s}}=0.48\right)$. The molecular structure of the cation of $\mathbf{1} \mathbf{b}$ is depicted 


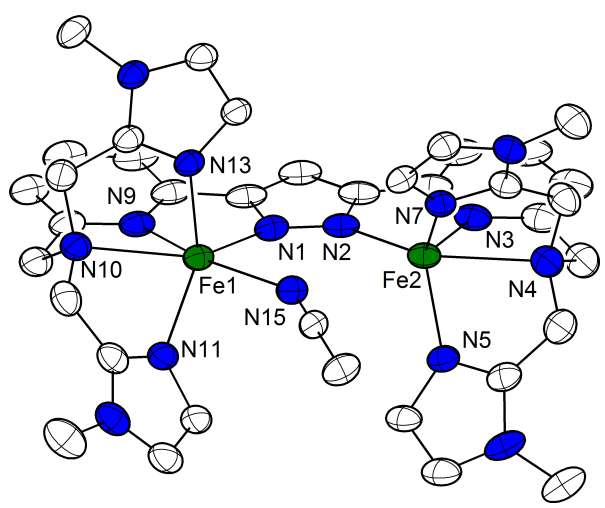

Figure 4.3: Crystal structure of the cation of $\left[\mathrm{L}^{\mathrm{Im}} \mathrm{Fe}\{\mathrm{Fe}(\mathrm{MeCN})\}\right]\left(\mathrm{BF}_{4}\right)_{3} \mathbf{1 b}$. Thermal ellipsoids are set to $30 \%$. Hydrogen atoms, solvent molecules as well as the $\mathrm{BF}_{4}^{-}$anions were omitted for clarity. Selected bond lengths $[\AA]$ and angles $\left[^{\circ}\right]$ Fe1-N1 2.109(3), Fe1-N9 2.185(3), Fe1N10 2.475(4), Fe1-N11 2.094(3), Fe1-N13 2.109(3), Fe1-N15 2.290(3), Fe2-N2 2.100(3), Fe2-N3 2.148(3), Fe2-N4 2.404(3), Fe2-N5 2.050(3), Fe2-N7 2.083(3), Fe1-N15-C200 148.1(3), Fe1-N1$\mathrm{N} 2-\mathrm{Fe} 25.668(6)$.

in Figure 4.3 .

As in 1a, the oxidation state of both iron atoms is $+\mathrm{II}$. The Fe $\cdots$ Fe distance is $4.38 \AA$. Despite the similar overall coordination of the iron centers in comparison with 1a, a detailed comparison of $\mathrm{Fe}-\mathrm{N}$ bond lengths indicates the same spin state for $\mathrm{Fe} 1$ and $\mathrm{Fe} 2$ in 1b. The spin state is most likely high spin for both iron centers as for a low spin state shorter $\mathrm{Fe}-\mathrm{N}$ distances would be expected. In order to find an explanation for this difference, close attention must be paid to changes of the coordination mode of the nitrile ligand. In $\mathbf{1 b}$ its coordination to Fe1 deviates significantly from linearity, exhibiting a bond angle of $148^{\circ}$. Also the Fe1-N10 bond in $\mathbf{1 b}$ is elongated compared to 1a. These two structural differences can possibly account for the spin state switch from low spin to high spin when going from $\mathbf{1 a}$ to $\mathbf{1} \mathbf{b}$. This behavior might be caused by the change of the counterion and the resulting crystal packing, as it will be discussed in more detail in Section 4.2.3. A detailed comparison of bond lengths and angles in $\mathbf{1 a}$ and $\mathbf{1} \mathbf{b}$ is given in Table 4.1 .

Table 4.1: Comparison of selected bond lengths and angles in $\mathbf{1 a}$ and $\mathbf{1 b}$.

\begin{tabular}{llcc}
\hline & Bond length $[\AA]$ & $\mathbf{1 a}$ & $\mathbf{1 b}$ \\
\hline 6-coordinate $\mathrm{Fe}$ & $\mathrm{Fe}-\mathrm{N}^{\mathrm{Pz}}$ & 1.948 & 2.109 \\
& $\mathrm{Fe}-\mathrm{N}^{\mathrm{MeCN}}$ & 1.938 & 2.290 \\
5-coordinate $\mathrm{Fe}$ & $\mathrm{Fe}-\mathrm{N}^{\mathrm{Pz}}$ & 2.071 & 2.100 \\
\hline & Bond angle [ $\left.{ }^{\circ}\right]$ & $\mathbf{1 a}$ & $\mathbf{1 b}$ \\
\hline & $\mathrm{Fe} 1-\mathrm{N} 1-\mathrm{N} 2-\mathrm{Fe} 2$ & 2.151 & 5.668 \\
& $\mathrm{Fe}-\mathrm{N}-\mathrm{C}^{\mathrm{MeCN}}$ & 174.8 & 148.1 \\
\hline
\end{tabular}

An overlay of the two cations of $\mathbf{1 a}$ and $\mathbf{1 b}$ (Figure 4.4) clearly illustrates the described structural differences. Apart from the different binding angle of the coordinating acetonitrile, the degree of distortion of the two iron sites varies, which is accompanied by significantly different $\mathrm{Fe}-\mathrm{N}$ bond lengths for the six-coordinate Fe atom. Moreover, the 
coordination of the imidazolyl side arms allows for some flexibility of the overall coordination geometry. Consequently, the methyl imidazole moieties are not stacked in the overlay (Figure 4.4), but adopt very different binding angles.

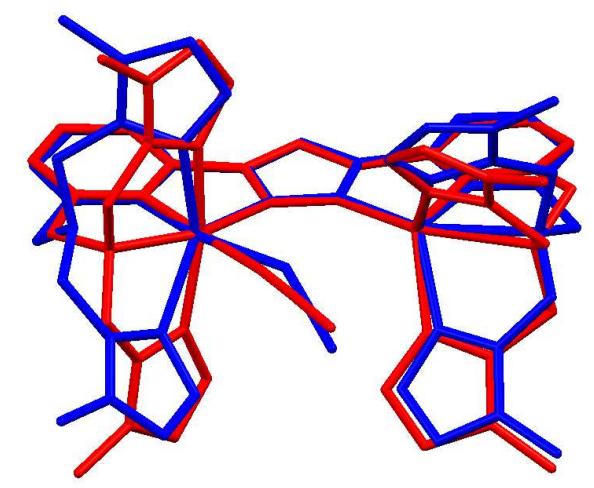

Figure 4.4: Structural overlay of the two cations of $\mathbf{1 a}$ (red) and $\mathbf{1 b}$ (blue).

\subsubsection{ESI Mass Spectrometry}

For verification of the integrity and stability of the obtained complexes $\mathbf{1 a}$ and $\mathbf{1} \mathbf{b}$ in solution ESI mass spectra were recorded. Under ionizing conditions the labile acetonitrile ligand in $\mathbf{1 a}$ and $\mathbf{1 b}$ is lost. Several twofold positively charged species become visible. These can be assigned to the complex cation with some monovalent anions that can be found in trace amounts in the spectrometer. Figure 4.5 depicts the obtained mass spectrum exemplary of 1a along with a simulation of the two largest signals.

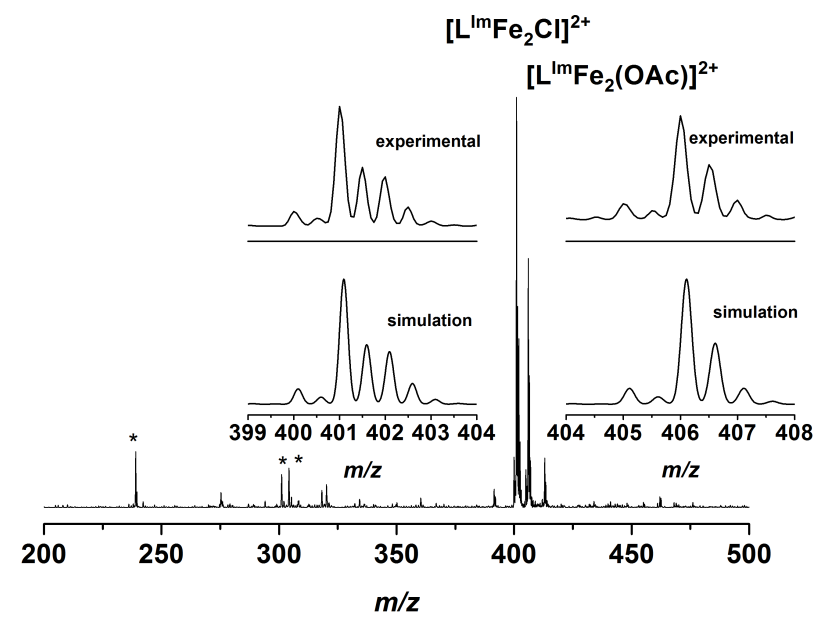

Figure 4.5: $\mathrm{ESI}^{+}$mass spectrum of 1a in MeCN. Comparison of experimental and simulated isotope pattern for the two main signals. Signals marked with * repesent common impurities from the spectrometer.

From ESI data it seems reasonable to assume that the complex cation does not undergo 
fragmentation under ionizing conditions. No organic ligand fragments were observed. A similar spectrum was obtained for $\mathbf{1 b}$. For details see Appendix 11.2.2.

\subsubsection{Mössbauer Spectroscopy}

Zerofield ${ }^{57} \mathrm{Fe}$ Mössbauer spectroscopy can serve as a valuable tool to elucidate oxidation states and spin states of iron atoms. Isomer shifts, i.e., the center of gravity in the spectrum can usually be empirically correlated to the oxidation and spin state of the iron site, and thus gives some indication of the electronic structure at the respective iron core. The quadrupol splitting of a signal, i.e., the separation of lines of a doublet, is caused by the electric charge distribution (electric field gradient EFG) at the iron center and can help to identify the spin state. Still, it is not only influenced by the spin state, but other factors such as symmetry and binding modes of the ligands. The zerofield Mössbauer spectra of $\mathbf{1 a}$ and $\mathbf{1 b}$ (Figure 4.6) exhibit two sets of signals.
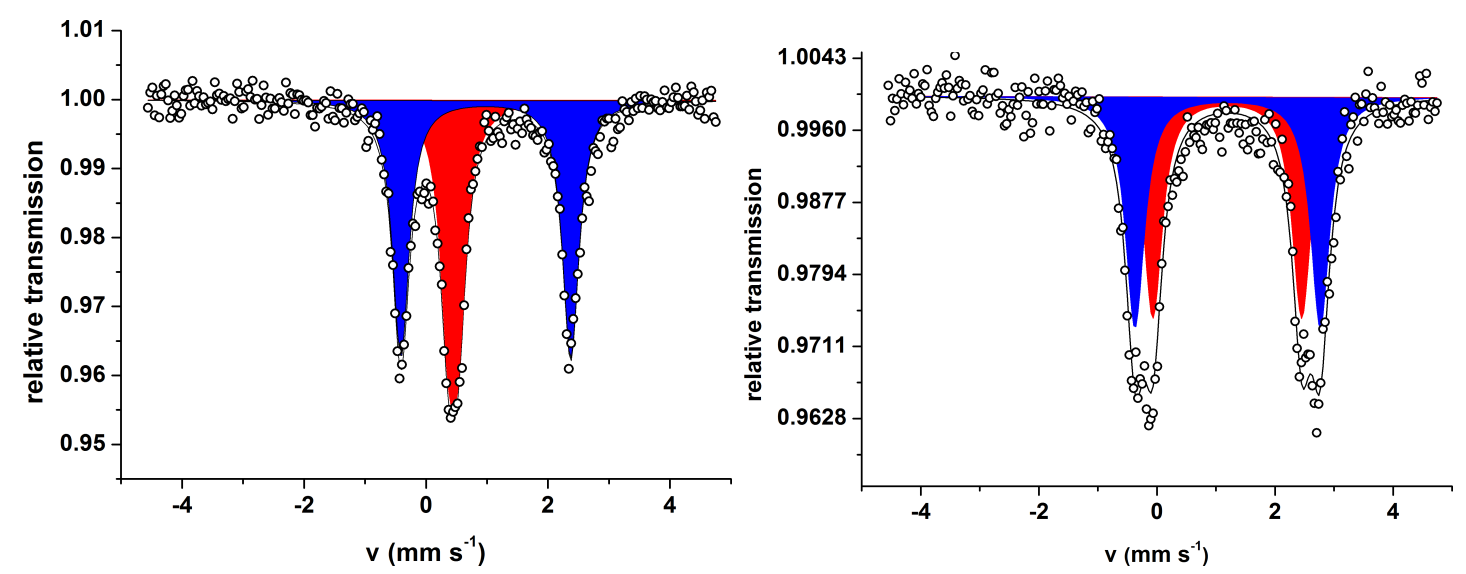

Figure 4.6: Left: Mössbauer spectrum of a crystalline sample of $\left[\mathrm{L}^{\mathrm{Im}} \mathrm{Fe}\{\mathrm{Fe}(\mathrm{MeCN})\}\right](\mathrm{OTf})_{3}$ 1a at $80 \mathrm{~K}$. Isomer shifts $\delta$ and quadrupole splittings $\left|\Delta E_{\mathrm{Q}}\right|$ in $\left[\mathrm{mm} \mathrm{s}^{-1}\right]$. Red subspectrum $\delta=0.45$, $\left|\Delta E_{\mathrm{Q}}\right|=0.16$, blue subspectrum $\delta=0.98,\left|\Delta E_{\mathrm{Q}}\right|=2.79$. Right: Mössbauer spectrum of a crystalline sample of $\left[\mathrm{L}^{\mathrm{Im}} \mathrm{Fe}\{\mathrm{Fe}(\mathrm{MeCN})\}\right]\left(\mathrm{BF}_{4}\right)_{3} \mathbf{1 b}$ at $80 \mathrm{~K}$. Isomer shifts $\delta$ and quadrupole splittings $\left|\Delta E_{\mathrm{Q}}\right|$ in $\left[\mathrm{mm} \mathrm{s}^{-1}\right]$. Red subspectrum $\delta=1.19,\left|\Delta E_{\mathrm{Q}}\right|=2.52$, blue subspectrum $\delta=1.20$, $\left|\Delta E_{\mathrm{Q}}\right|=3.14$.

The isomer shifts of all signals lie within the scope for Fe(II) species. While the spectrum for 1a exhibits one Mössbauer doublet with a very large (blue) and a second with a very small quadrupole splitting (red), $\mathbf{1 b}$ exhibits two doublets with large splitting parameters. The larger quadrupole splitting parameters can be assigned to high spin species whereas the very small splitting indicates a low spin species in $\mathbf{1 a}$.

Thus the previously discussed spin state considerations based on crystallographic data can be confirmed by this technique. In solid crystalline samples of $\mathbf{1 a}$ one iron ion is low spin and one high spin. For $\mathbf{1 b}$, two high spin iron ions are observed which is in agreement with previous findings from structural analysis.

\subsubsection{Anion Effects in the Solid State}

Since the cations of $\mathbf{1 a}$ and $\mathbf{1} \mathbf{b}$ differ structurally in the binding angle of the coligand acetonitrile and electronically by a change from low spin to high spin for the six-coordinate 
iron atom, it seems feasible to discuss possible effects of the counterions on the core structure of the complex. Anions are known to exhibit a noticeable influence on the crystal packing and structural parameters, and thus on the spin states in certain materials. According to SPIERING et al. even small changes in the ligands and in the second coordination shell as well as cocrystallized solvent can shift the thermal spin transition to very different temperatures or even suppress it entirely. 140] The anion volume of monovalent anions seems to exhibit a significant effect on the SCO properties of the cationic metal complex. The transition temperature was found to increase with smaller volumes of spherical anions in coordination polymers with $\left\{\mathrm{N}_{6}\right\}$ coordinated Fe(II) sites. $\frac{143144}{14}$ These findings would suggest that at comparable temperatures smaller anions support a high spin state, whereas larger anions might favor a low spin state. In the particular example of $\mathbf{1 a}$ and $\mathbf{1 b}$, the $\mathrm{BF}_{4}^{-}$anion is considerably smaller than the $\mathrm{OTf}^{-}$anion which could contribute to an explanation for the spin state switch. 145]

From the crystal packings of $\mathbf{1 a}$ and $\mathbf{1 b}$ no particular symmetrical arrangement of the anions in the crystal lattice could be observed. Moreover $\mathrm{OTf}^{-}$and $\mathrm{BF}_{4}^{-}$are generally capable to form intermolecular networks through hydrogen bonds. Hydrogen bonds as well as $\pi$-stacking effects can additionally contribute to spin transitions in the solid state. 136 . In the structures at hand no hydrogen bonds were found within the crystal lattice. Due to the fact that the aprotic solvent $\mathrm{MeCN}$ was used these findings are not surprising. However, for $\mathbf{1 b}$ hydrogen bond contributions cannot entirely be ruled out as residual electron density was omitted for final structural refinement.

Pronounced anion effects have been observed for pyrazolate based [2×2] grid complexes with $\mathrm{Fe}(\mathrm{II})$. The work of SteIneRT supports the theory that anions as well as solvent molecules exhibit influences on crystal packing and consequently on the octahedral coordination of the iron sites. $124[134$

\subsection{Spin Crossover of Diferrous Complexes of $\mathrm{HL}^{\mathrm{Im}}$ in Solid State and Solution}

As subtle structural and intermolecular changes in the crystalline material were found to alter the spin state of the six-fold coordinated iron atom in the diiron complexes from low spin in $\mathbf{1 a}$ to high spin in $\mathbf{1} \mathbf{b}$, it seems feasible to investigate the potential occurrence of a SCO phenomenon in the respective compounds. The current study focuses on triggering a potential SCO by variation of the temperature. A valuable tool in this respect are magnetic measurements to determine the temperature dependent magnetic moment by, e.g., SQUID magnetometry.

In this chapter the SCO in the solid state in particular for $\mathbf{1 a}$ will be elucidated.

Moreover the solid state phenomenon is compared to the behavior of $\mathbf{1 a}$ and $\mathbf{1} \mathbf{b}$ in solution. Solution studies were conducted via UV/Vis spectroscopy, supported by Mössbauer and SQUID measurements. 


\subsubsection{SCO of 1a in Solid State}

Focussing on 1a the following considerations have to be taken into account: The diiron core of 1a in the molecular structure hosts a Fe(II) high spin and a Fe(II) low spin site. The crystallographic data were collected at $133 \mathrm{~K}$, and thus the structurally derived spin states represent the electronic configuration at this temperature. Consequently, a potential spin transition to two Fe(II) high spin sites has to occur above $133 \mathrm{~K}$. The previously discussed Mössbauer spectrum of $\mathbf{1 a}$ has been recorded at $80 \mathrm{~K}$. It is in good agreement with the spin states assigned from structural considerations at $133 \mathrm{~K}$. This indicates that the spin state does not change in the range of $80 \mathrm{~K}$ and $133 \mathrm{~K}$. To rule out a spin transition below $80 \mathrm{~K}$ the Mössbauer temperature was lowered to $6 \mathrm{~K}$. However, no spin transition to two $\mathrm{Fe}(\mathrm{II})$ low spin was observed upon cooling. Consequently, only the temperature range above $133 \mathrm{~K}$ is relevant for the observation of possible SCO phenomena. The respective Mössbauer spectra of the $6 \mathrm{~K}$ measurement can be found in Appendix 11.2.3 Figure 11.5. For a more detailed insight into the magnetic behavior of 1a SQUID data were collected.

\section{SQUID Magnetometry}

SQUID magnetometry allows for a measurement of small changes of the magnetization of a sample with respect to the temperature. Even small amounts of a magnetic material are sufficient to obtain reliable information. The sample preparation, however, can be challenging. A common solid SQUID sample is placed in a gel capsule and sealed with a lid. The entire capsule is placed in the middle of a PTFE straw and mounted in the magnetometer. To avoid any magnetic orientation and thus rearrangement of the material inside the magnetometer the sample can be covered with a drop of fluorinated oil that prevents movements inside the gel capsule. Crystalline samples of 1a proved to be very sensitive to solvent loss. This behavior was discovered when drying crystals of $1 \mathbf{a}$ in vacuo. Upon drying both Fe(II) sites become high spin. The respective Mössbauer spectrum at $80 \mathrm{~K}$ can be found in Appendix 11.2.3, Figure 11.6. To circumvent solvent loss for the duration of a SQUID measurement, sample preparation had to be modified. Crystals of 1a were placed together with mother liquid from the crystallization in a glass NMR tube. Crystals were fixed inside the tube with a stopper of teflon ribbon. The supernatant solution was decanted. The tube was sealed through melting of the upper part of the glass tube. The obtained SQUID data are depicted in Figure 4.7.

The product of temperature and the magnetic susceptibility appears to be constant over a wide temperature range. The value of $\chi_{\mathrm{M}} T$ of $3.14 \mathrm{~cm}^{3} \mathrm{~K} \mathrm{~mol}^{-1}$ corresponds to the expected value for one Fe(II) high spin site. The slope of the data points starts to change at temperatures above $210 \mathrm{~K}$. The S-shaped curve reflects a typical spin crossover pattern. At $300 \mathrm{~K}$ the spin state change is not fully evolved which would be manifested in the occurrence of a second temperature plateau. However, in solid state the crossover is very abrupt. The SCO temperature lies around $264 \mathrm{~K}$, which is marked by the point of inflection of the $\mathrm{S}$-shaped curve. This value is in perfect agreement with SCO temperatures for similar literature known dinuclear pyrazolate based Fe(II) SCO complexes. 146 149] The decrease of $\chi_{\mathrm{M}} T$ below $50 \mathrm{~K}$ can be attributed to zerofield splitting effects $\left(D=-10.81 \mathrm{~cm}^{-1}\right)$. All data were corrected for temperature independent paramagnetism (TIP). A hysteretic be- 


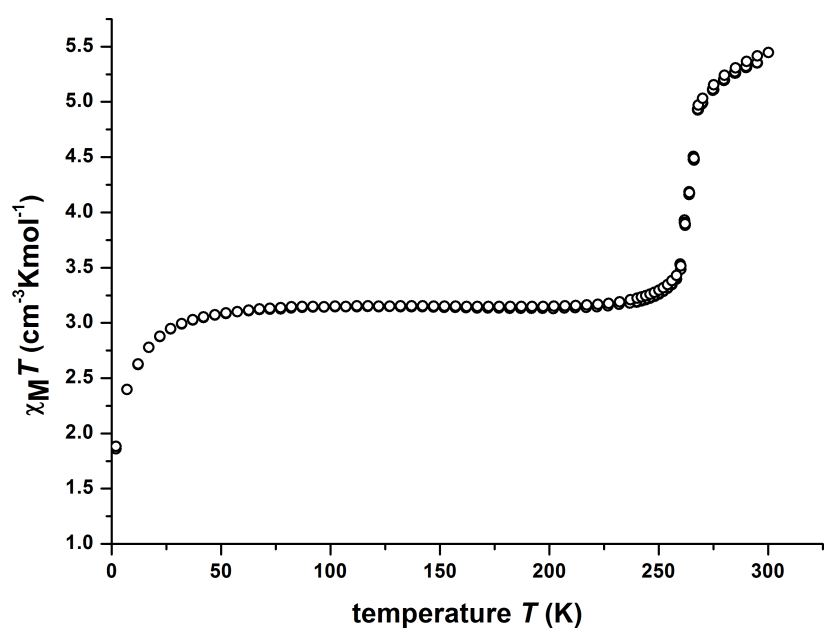

Figure 4.7: SQUID magnetometric measurement of a crystalline sample of $\mathbf{1 a}$ in mother liquid. Spin crossover from one Fe(II)ls and one Fe(II)hs to two Fe(II)hs occurs in the range of $210 \mathrm{~K}$ to $300 \mathrm{~K}$.

havior as observed for molecular storage devices, in which the magnetization behavior during the gradual warming and cooling processes differs, was not observed for $\mathbf{1 a}$.

\subsubsection{SCO of 1a in Solution}

In solution spin transitions of molecules have been rarely investigated. 135. A spin transition in solution, similar as in solid state, requires that the potential minima of the high spin and low spin surface are very close in energy so that both can be populated. 150 However in solution the SCO is mostly more gradual and can be accompanied by a variety of processes such as ligand exchange reactions or coordination/decoordination of chelating side arms of a multidentate ligand. $135 \mid 151$.

To probe the SCO phenomenon of $\mathbf{1 a}$ in solution, a variety of techniques can be applied. Frozen solution Mössbauer spectroscopy in various solvents can help to elucidate the spin state at a certain temperature. Of particular interest are nitrile solutions of the respective complex, since coordination/decoordination of the nitrile coligand might have a substantial influence on spin state changes in solution. Furthermore temperature dependent UV/Vis and NMR techniques allow for a more gradual spectroscopic examination of the solution behavior. It should be noted though, that UV/Vis and NMR techniques are limited to the freezing point of the respective solvent. Opposed to frozen solution Mössbauer measurements, these methods can only be conducted with liquid samples. Moreover SQUID additional solution measurements can be conducted for a closer insight into the nature of the spin transition.

The behavior of $\mathbf{1 a}$ in solution is discussed in this section. However as solid state effects e.g., crystal packings and anion effects should not contribute to SCO phenomena in solution it can be assumed that $\mathbf{1 a}$ and $\mathbf{1} \mathbf{b}$ display similar properties. 


\section{UV/Vis Spectroscopy}

UV/Vis spectra were recorded on a spectrometer equipped with a cryostat which allowed gradual cooling of the sample. Samples of 1a were dissolved in dry freeze-pump-thawed degassed solvents in a glovebox and transferred to a Schlenk cuvette equipped with a stirring bar. The cuvette was sealed air tight. Figure 4.8 depicts the temperature dependent $\mathrm{UV} / \mathrm{V}$ is spectrum of 1a in EtCN. EtCN does not freeze until temperatures of $-92{ }^{\circ} \mathrm{C}$ and is therefore suitable for low temperature UV/Vis experiments. Very pronounced changes in the absorption can be observed for bands of wavelengths around $417 \mathrm{~nm}$. A general observation in literature is that Fe(II) low spin states exhibit higher extinction coefficients than their corresponding high spin analogues. $135 \mid 152$ In that respect upon lowering the temperature the low spin character in solutions of $\mathbf{1 a}$ would increase.

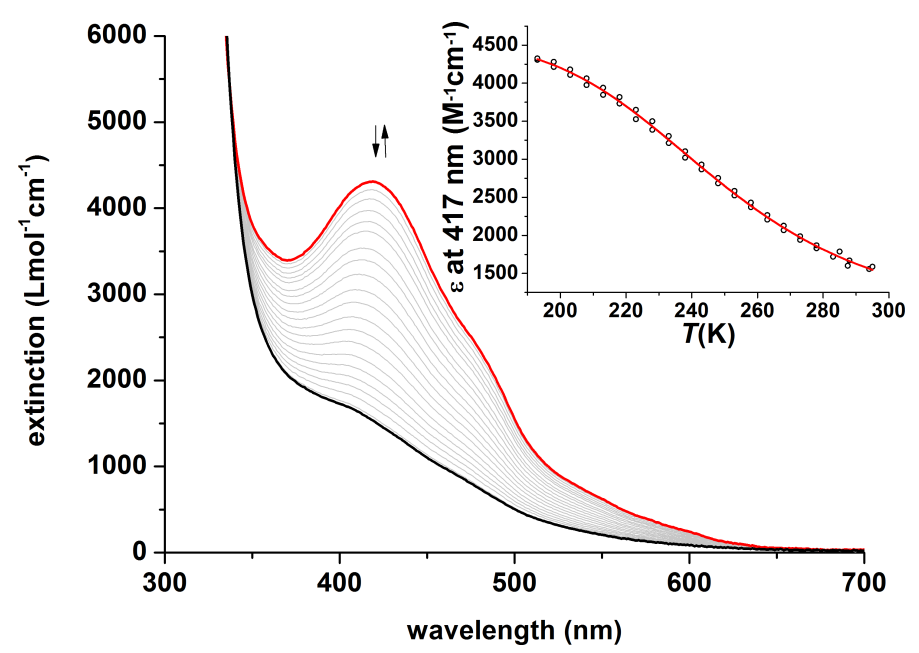

Figure 4.8: Excerpt of the variable temperature UV/Vis spectrum of 1a in EtCN. The arrows indicate stepwise cooling to $-80{ }^{\circ} \mathrm{C}$ and subsequent warming to ambient temperature. Inset: temperature trace of the extinction coefficient at $417 \mathrm{~nm}$ with fit according to Equation 4.3 .

The temperature trace of the bands at $417 \mathrm{~nm}$ yielded an S-shaped curve which could be fit according to the following considerations. The temperature dependence of the experimentally obtained $\epsilon$ values is attributed to the change of the mole fraction of molecules in the high spin state $\left(\gamma_{\mathrm{HS}}\right)$.

$$
\gamma_{\mathrm{HS}}=\frac{\epsilon-\epsilon_{\mathrm{LS}}}{\epsilon_{\mathrm{HS}}-\epsilon_{\mathrm{LS}}} \quad \text { or } \quad \epsilon=\epsilon_{\mathrm{LS}}+\gamma_{\mathrm{HS}}\left(\epsilon_{\mathrm{HS}}-\epsilon_{\mathrm{LS}}\right)
$$

$\epsilon_{\mathrm{HS}}$ and $\epsilon_{\mathrm{LS}}$ describe the molar extinction coefficients of the pure high spin and low spin states in this respect. The ideal solution model for the mole fraction of molecules in the high spin state is described by the following equation: $153 \mid 154$

$$
\gamma_{\mathrm{HS}}=\frac{1}{1+\mathrm{e}^{(\Delta H / \mathrm{R} T-\Delta S / \mathrm{R})}}
$$

Combining these equations an analytical expression for the temperature dependence of the extinction coefficient $\epsilon$ can be derived: 


$$
\epsilon=\epsilon_{\mathrm{LS}}+\frac{1}{1+\mathrm{e}^{(\Delta H / \mathrm{R} T-\Delta S / \mathrm{R})}}\left(\epsilon_{\mathrm{HS}}-\epsilon_{\mathrm{LS}}\right)
$$

Since the transition is incomplete the molar extinction coefficients $\epsilon_{\mathrm{HS}}$ and $\epsilon_{\mathrm{LS}}$ had to be determined by a fit using Equation 4.3 . Together with the thermodynamic parameters $\Delta H$ and $\Delta S$ four parameters had to be adjusted. The best fit was obtained with the parameters $\Delta H=19.1 \mathrm{~kJ} \mathrm{~mol}^{-1}, \Delta S=77 \mathrm{~J} \mathrm{~mol}^{-1} \mathrm{~K}^{-1}, \epsilon_{\mathrm{HS}}=850 \mathrm{~cm}^{2} \mathrm{mmol}^{-1}$ and $\epsilon_{\mathrm{LS}}=4560 \mathrm{~cm}^{2} \mathrm{mmol}^{-1}$. The obtained $\Delta H$ and $\Delta S$ values lie in the typical range for Fe(II)

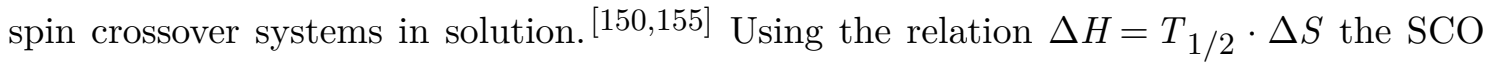
temperature can be determined to $T_{1 / 2}=248 \mathrm{~K}$ in EtCN. With the respective $\epsilon_{\mathrm{HS}}$ and $\epsilon_{\mathrm{LS}}$ values the mole fraction of molecules in the high spin state at a given temperature can be calculated according to Equation 4.1.

Figure 4.9 depicts the fit of the mole fraction of molecules in the high spin state in dependence of the temperature.

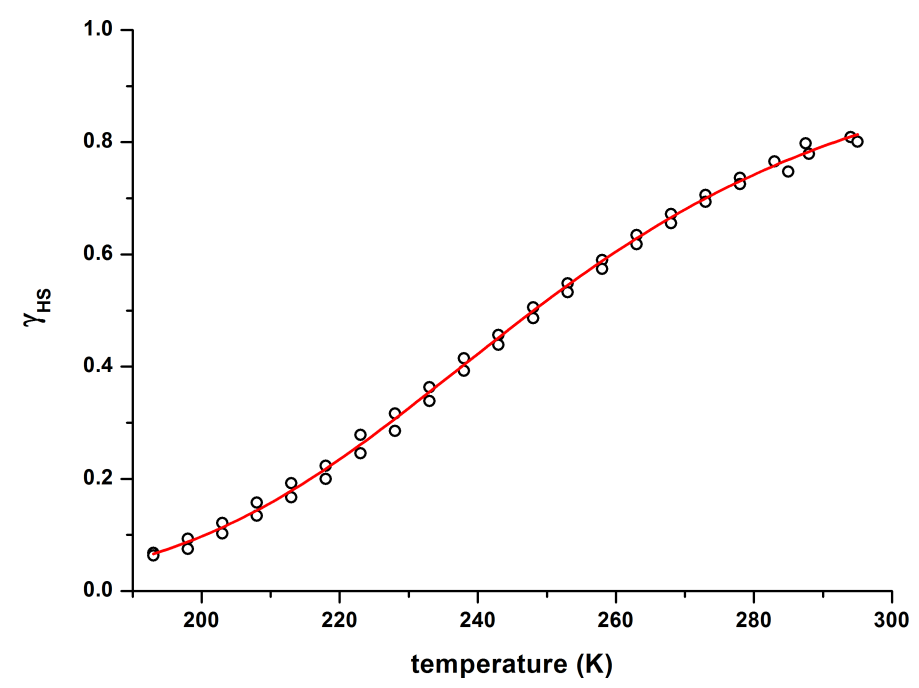

Figure 4.9: Temperature dependence of the mole fraction of molecules in the high spin state in EtCN solution.

Thus for 1a in EtCN solution a gradual SCO can be assumed. The transition is incomplete due to the limited range in which the solvent EtCN does not freeze.

For reasons of comparison the same experiment was conducted in MeCN at variable temperatures. MeCN freezes at $-45{ }^{\circ} \mathrm{C}$. The temperature range of the measurement is therefore even more limited. Figure 4.10 depicts the respective spectrum.

The shape of the band at $417 \mathrm{~nm}$ is very similar compared to the respective spectrum in EtCN. The temperature trace was fit according to Equation 4.3. The slope represents a rather linear dependency of the absorbance with temperature in the investigated range. The best fit was obtained with the following parameters: $\Delta H=23.5 \mathrm{~kJ} \mathrm{~mol}^{-1}$, $\Delta S=86 \mathrm{~J} \mathrm{~mol}^{-1} \mathrm{~K}^{-1}, \epsilon_{\mathrm{HS}}=860 \mathrm{~cm}^{2} \mathrm{mmol}^{-1}$ and $\epsilon_{\mathrm{LS}}=2000 \mathrm{~cm}^{2} \mathrm{mmol}^{-1}$. Also these obtained $\Delta H$ and $\Delta S$ values lie in the typical range for $\mathrm{Fe}(\mathrm{II})$ spin crossover systems in solution. 150[155] The SCO temperature in MeCN was calculated to $T_{1 / 2}=273 \mathrm{~K}$. The plot of the mole fraction of molecules in the high spin states resulting from these relations is shown in Figure 4.11 . 


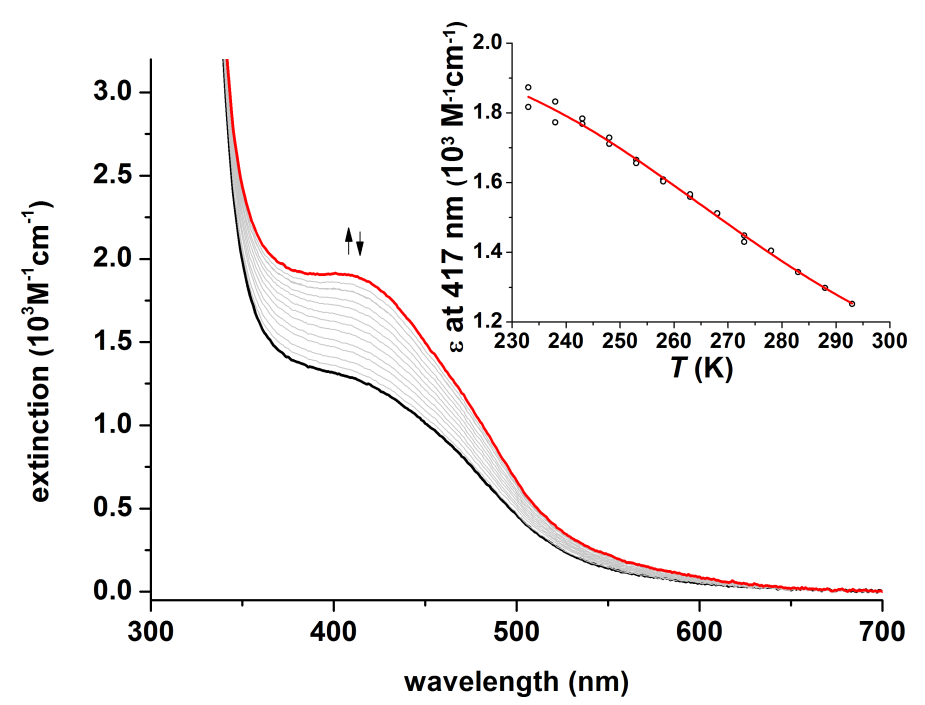

Figure 4.10: Excerpt of the variable temperature UV/Vis spectrum of 1 a in MeCN. The arrows indicate the stepwise cooling process to $-40^{\circ} \mathrm{C}$ and subsequent warming to ambient temperature. Inset: temperature trace of the extinction coefficient at $417 \mathrm{~nm}$ with fit according to Equation 4.3 .

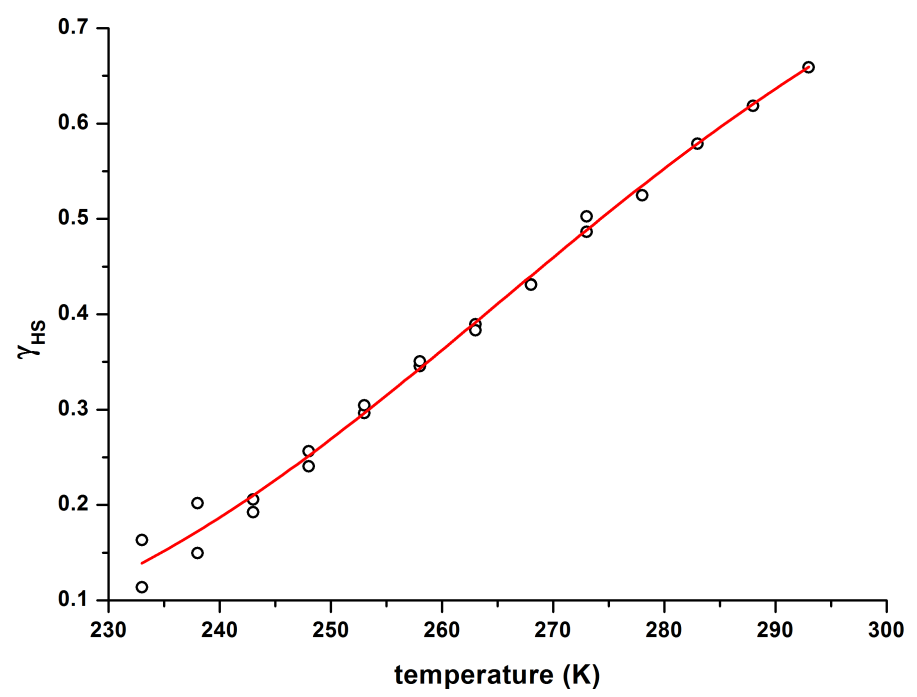

Figure 4.11: Temperature dependence of the mole fraction of molecules in the high spin state in MeCN solution.

From UV/Vis spectra only it is yet unclear if the temperature dependency of the band at $417 \mathrm{~nm}$ does correspond to an SCO phenomenon in solution or if it can be also associated with different processes. An option that should be considered is a ligand exchange equilibrium of the respective nitrile. For a standard equilibrium between two species a Van't Hoff relationship would yield similar thermodynamic parameters.

A suitable experiment to rule out ligand exchange equilibria with the respective nitrile would involve the sequential addition of nitrile to a solution of $1 \mathbf{a}$ in a non coordinating solvent such as DCM at low temperatures. This experiment was conducted twice. However, as solutions of $\mathbf{1 a}$ are very sensitive to dioxygen and thus air, difficulties were experienced in a proper air tight setup for the experiments. Even in sealed cuvettes with 
a septum a slow diffusion of dioxygen into the solution could not be avoided.

\section{SQUID Magnetometry}

One of the most versatile and reliable methods to follow a gradual SCO in solution is SQUID magnetometry. Samples were prepared in a similar manner as for solid state measurements. In a glovebox, crystalline material of 1a was dissolved in a defined volume of dry MeCN. The complex solution was transferred in a NMR tube and sealed. The upper part of the glass tube was sealed to prevent oxidation of the sample solution. The measurement was corrected for temperature independent paramagnetism (TIP) and by paramagnetic contributions of the glass tube. The obtained spectrum is depicted in Figure 4.12 .

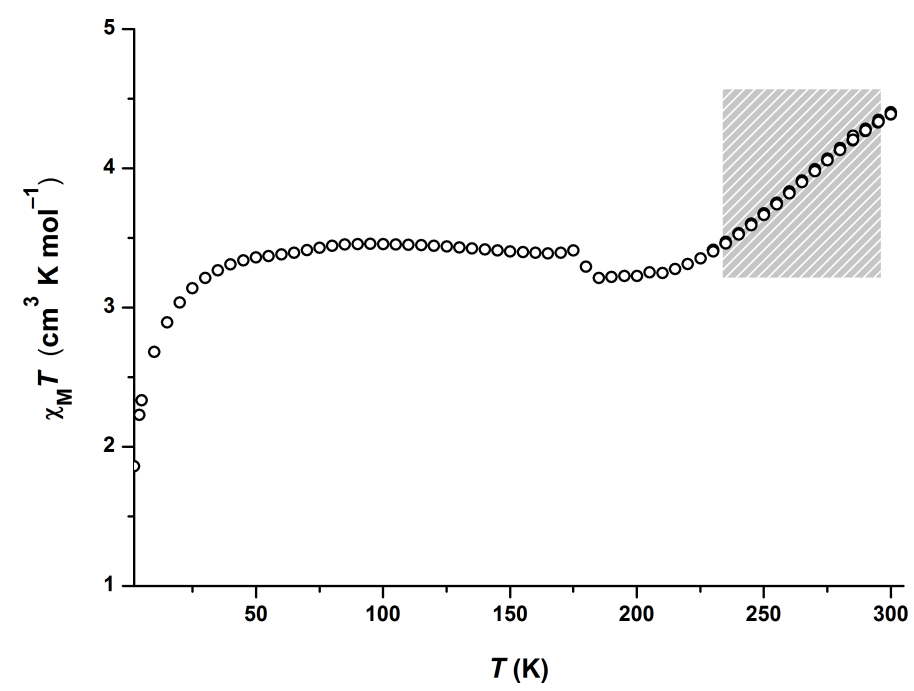

Figure 4.12: $\chi_{\mathrm{M}} T$ vs. $T$ plot for a solution of 1a in MeCN. The highlighted area marks the temperature regime in which $\mathrm{UV} / \mathrm{V}$ is data are available (compare Figure 4.10 .

Over a wide temperature range the magnetization and thus susceptibility of the sample is constant and in the expected range for one Fe(II) low spin and one high spin ion. A gradual increase can be observed in the temperature range from $210 \mathrm{~K}$ to ambient temperature. The gray highlighted area indicates the temperature regime which can be compared to $\mathrm{UV} / \mathrm{Vis}$ data (Figure 4.10). In this regime the gradual increase is almost linear which matches the findings from UV/Vis data. With increasing high spin character of the second iron atom the UV/Vis absorbance at $417 \mathrm{~nm}$ decreases and vice versa. To compare UV/Vis and SQUID data the obtained magnetic susceptibilities can be fit according to the following equation to extract thermodynamic parameters:

$$
\left.\chi_{\mathrm{M}} T=\left(\chi_{\mathrm{M}} T\right)_{\mathrm{LS}}+\frac{1}{1+\mathrm{e}^{(\Delta H / \mathrm{R} T-\Delta S / \mathrm{R})}}\left(\left(\chi_{\mathrm{M}} T\right)_{\mathrm{HS}}-\left(\chi_{\mathrm{M}} T\right)_{\mathrm{LS}}\right)\right)
$$

The best fit was obtained using the parameters $\Delta H=24.2 \mathrm{~kJ} \mathrm{~mol}^{-1}, \Delta S=89 \mathrm{~J} \mathrm{~mol}^{-1} \mathrm{~K}^{-1}$, $\left(\chi_{\mathrm{M}} T\right)_{\mathrm{HS}}=4.81 \mathrm{~cm}^{3} \mathrm{mmol}^{-1} \mathrm{~K}$ and $\left(\chi_{\mathrm{M}} T_{\mathrm{LS}}=2000 \mathrm{~cm}^{3} \mathrm{mmol}^{-1} \mathrm{~K}\right.$. The thermodynamic data of both UV/Vis and SQUID data are in excellent agreement. The fit of the SQUID data according to Equation 4.4 and a comparison of the mole fraction of molecules in the 
high spin state of both measurements can be seen in Figure 4.13
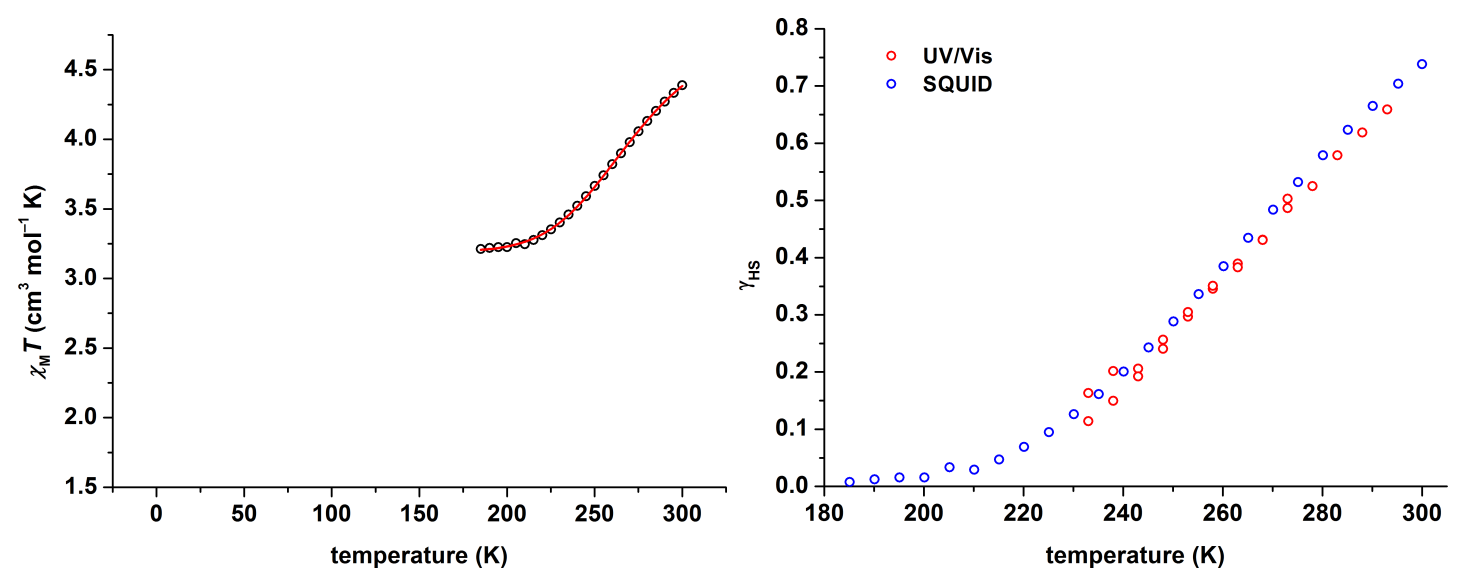

Figure 4.13: Left: Excerpt of SQUID data fitted according to Equation 4.4 Right: Comparison of the mole fraction of molecules in the high spin state from UV/Vis measurements in MeCN (red circles) and SQUID data (blue circles).

SQUID and UV/Vis measurement of $\mathbf{1 a}$ in solution both indicate a gradual spin crossover from $\mathrm{Fe}(\mathrm{II})$ ls $\mathrm{Fe}$ (II)hs to two Fe(II)hs. Both measurements in MeCN are in good agreement. However, the SCO phenomenon is strongly solvent dependent and varies even within nitrile solutions. To date it has not been possible to exclude ligand exchange equilibria with the respective nitrile which might account for the phenomenon.

\section{Solution Mössbauer Spectra}

Another elaborate understanding of the spin state of $\mathbf{1 a}$ in solution at low temperatures can be gained from frozen solution Mössbauer spectroscopy.

For solution Mössbauer measurements of 1a, crystalline material was dissolved in $1 \mathrm{~mL}$ of the respective solvent $(\mathrm{c} \sim 15 \mathrm{mM})$. This solution was transferred to a sample holder in a glovebox. The entire sample holder was immediately flashfrozen in liquid nitrogen when removed from the nitrogen atmosphere of the glovebox. The respective spectra were recorded at $80 \mathrm{~K}$ and are depicted in Figure 4.14.

The immediate freezing process is expected to prevent any significant packing- and anion effects. Figure 4.14 suggests two Mössbauer subspectra for each solution measurement of 1a. Isomer shifts and quadrupole splittings are comparable to those found for the solid state structure of 1a. In Table 4.2 all relevant parameters are compared.

Table 4.2: Comparison of Mössbauer parameters for $\mathbf{1 a}$.

\begin{tabular}{c|ll|ll|ll}
\hline & crystalline 1a & $(80 \mathrm{~K})$ & 1a in EtCN & $(80 \mathrm{~K})$ & 1a in MeCN & $(80 \mathrm{~K})$ \\
\hline$\delta\left[\mathrm{mm} \mathrm{s}^{-1}\right]$ & 0.45 & 0.98 & 0.43 & 1.00 & 0.40 & 1.01 \\
$\left|\Delta E_{\mathrm{Q}}\right|\left[\mathrm{mm} \mathrm{s}^{-1}\right]$ & 0.16 & 2.79 & 0.20 & 2.92 & 0.10 & 2.88 \\
\hline
\end{tabular}

At $80 \mathrm{~K}$ 1a displays a high spin and a low spin $\mathrm{Fe}$ (II) signal in solid state and solution. If the process followed by UV/Vis measurement marks a SCO, the only spin transition that may occur is a spin flip of one Fe(II) ion from high spin to low spin upon cooling. 

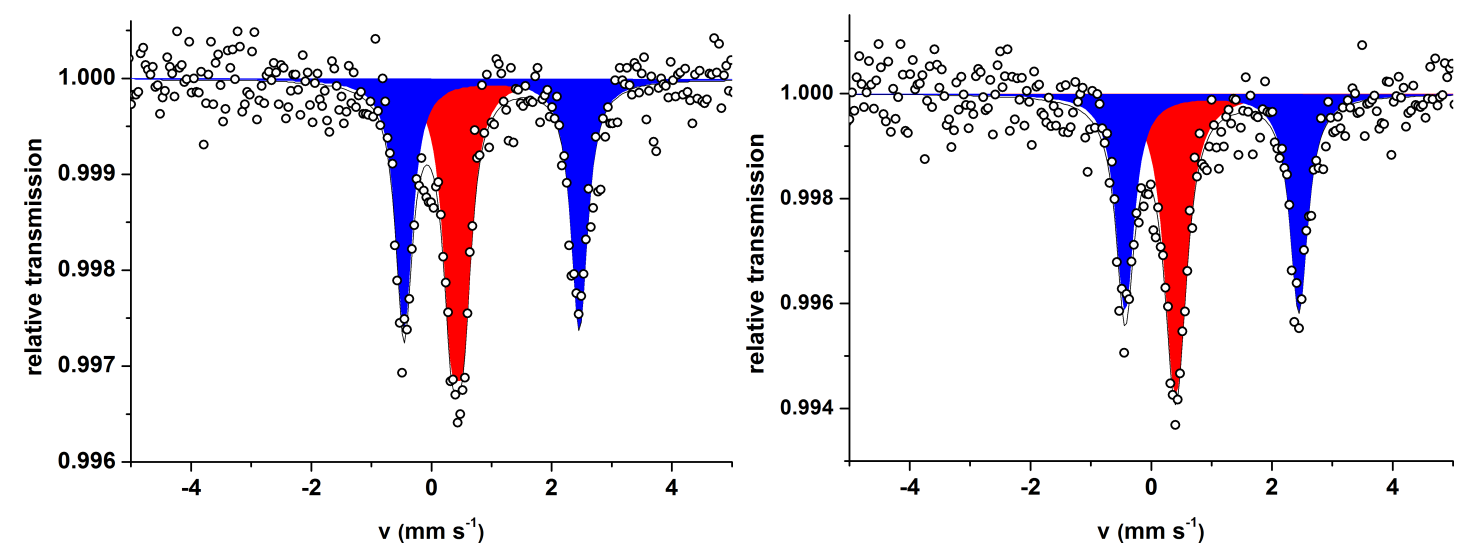

Figure 4.14: Zerofield Mössbauer spectra of solutions of $1 \mathbf{a}$ in EtCN and MeCN at $80 \mathrm{~K}$. Isomer shifts $\delta$ and quadrupole splittings $\left|\Delta E_{\mathrm{Q}}\right|$ in $\left[\mathrm{mm} \mathrm{s}^{-1}\right]$. Left: Spectrum in EtCN. Red subspectrum $\delta=0.43,\left|\Delta E_{\mathrm{Q}}\right|=0.20$, blue subspectrum $\delta=1.00,\left|\Delta E_{\mathrm{Q}}\right|=2.92$. Right: Spectrum in MeCN. Isomer shifts $\delta$ and quadrupole splittings $\left|\Delta E_{\mathrm{Q}}\right|$ in $\left[\mathrm{mm} \mathrm{s}^{-1}\right]$. Red subspectrum $\delta=0.40,\left|\Delta E_{\mathrm{Q}}\right|=0.10$, blue subspectrum $\delta=1.01,\left|\Delta E_{\mathrm{Q}}\right|=2.88$.

For $\mathbf{1 b}$ according solid state and solution Mössbauer spectra were recorded. The spectra can be found in Appendix 11.2.3 Figure 11.7. These spectra, however, did not lead to coherent results as found for 1a. The spectra display three subspectra for samples in frozen EtCN solutions, which may be explained by an incomplete spin transition upon freezing and /or the coordination of the nitrile in different binding angles upon freezing of the sample. In frozen MeCN solutions two Fe(II) subspectra are observed in a 76:24 ratio (Appendix 11.2.3 Figure 11.8). Besides the possibility of impurities in the sample, no matching explanation for this behavior has been found to date.

\section{Evans NMR Method}

A possibility to confirm the obtained magnetic data from SQUID measurements is a variable temperature NMR analysis with the Evans method. The Evans method describes a concept to measure the magnetic susceptibility of a paramagnetic sample via the temperature dependent shift of NMR signals. The chemical shift of an inert compound such as a solvent or different internal reference varies in the presence of a paramagnetic material. 156 .

Samples for the Evans measurement were prepared according to the following procedure: In a glovebox a defined amount of sample was dissolved in $0.3 \mathrm{~mL}$ dry $\mathrm{MeCN}^{-} \mathrm{d}_{3}$ and placed in an NMR tube. A coaxial reference tube was filled with a blank of the same solvent. This reference tube was placed inside the NMR tube. Both tubes were sealed air tight and submitted for the variable temperature ${ }^{1} \mathrm{H}-\mathrm{NMR}$ measurement. Starting from ambient temperature the gradual decrease of temperature by $10^{\circ} \mathrm{C}$ caused a shift of the solvent signals for the paramagnetic solution, while signals assigned to the reference remained constant.

An excerpt of the according NMR spectra is depicted in Figure 4.15 .

With the respective difference of the solvent signals the magnetic susceptibility was cal- 


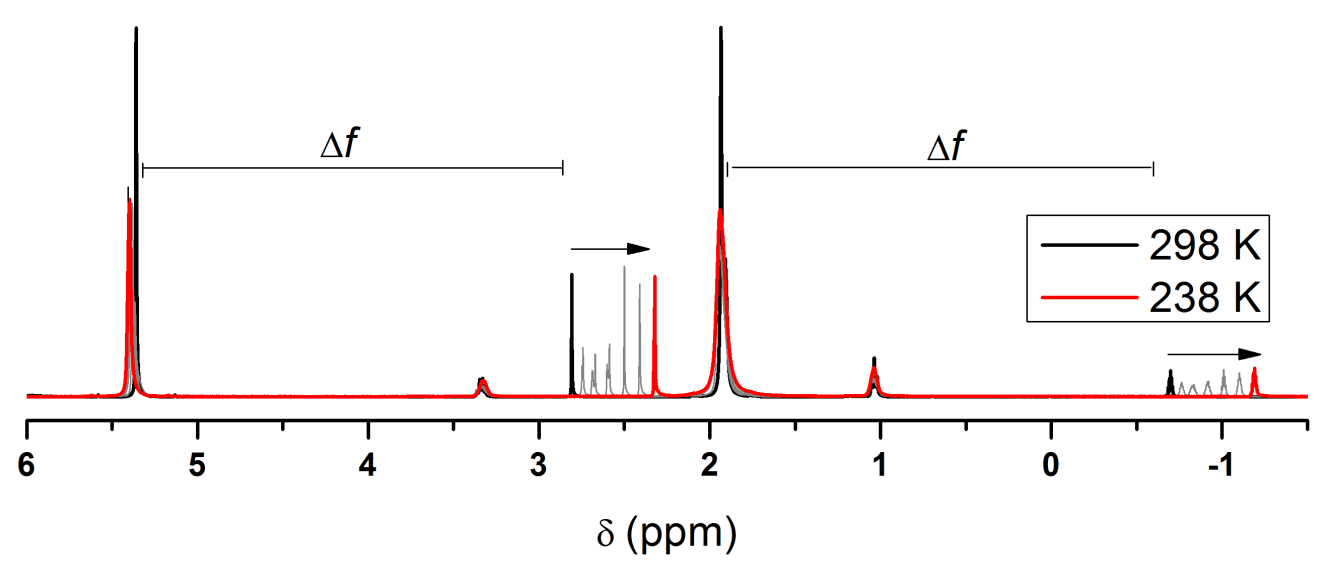

Figure 4.15: Evans ${ }^{1} \mathrm{H}$ NMR measurement in dry MeCN- $\mathrm{d}_{3}$. Gradual decrease of the temperature by $10^{\circ} \mathrm{C}$ accompanied by chemical shifts of the solvent. NMR shifts were not referenced according to common procedures as it its unknown which set of signals corresponds to the inner or outer tube. The relative difference between these signals is of relevance for the determination of the magnetic susceptibility as indicated by $\Delta f$.

culated according to Equation 4.5 .

$$
\chi_{\mathrm{g}}=\frac{3 \Delta f}{2 \pi f m}+\chi_{0}+\frac{\chi_{0}\left(\mathrm{~d}_{0}-\mathrm{d}_{\mathrm{s}}\right)}{m}
$$

Table 4.3: Legend for Equation 4.5156

$\chi_{\mathrm{g}}$ mass susceptibility of the sample solution $\left[\mathrm{cm}^{3} / \mathrm{g}\right]$

$\Delta f \quad$ frequency shift between two sets of signals $[\mathrm{Hz}]$

$f \quad$ spectrometer frequency $[\mathrm{Hz}]$

$\chi_{0} \quad$ mass susceptibilitv of solvent $\left[\mathrm{cm}^{3} / \mathrm{g}\right]$

$m$ mass of substance per $\mathrm{cm}^{3}$ of solution

$\mathrm{d}_{0}$ density of the solvent $\left[\mathrm{g} / \mathrm{cm}^{3}\right]$

$\mathrm{d}_{\mathrm{S}}$ density of the sample solution $\left[\mathrm{g} / \mathrm{cm}^{3}\right]$

The main two variables in the described equation are the temperature dependent frequency shift, which can be obtained from the recorded spectrum and the changing density with the temperature of the solution. The densities were assumed constant for the investigated temperature range as the difference of densities should not change significantly.

Figure 4.16 depicts the $\chi_{\mathrm{M}} T$ vs. $T$ plot of the obtained data.

The product of susceptibility and temperature varies between 3.9 and $4.2 \mathrm{~cm}^{3} \mathrm{Kmol}^{-1}$ and decreases with lower temperatures. This reflects the trend of the recorded data from $\mathrm{UV} / \mathrm{V}$ is and SQUID measurements. The increase is almost linear as expected. Solely the determined $\chi_{\mathrm{M}} T$ values differ from the ones obtained by SQUID measurements in the experimentally accessible temperature range. From SQUID data an increase from approximately 3.1 to $4.5 \mathrm{~cm}^{3} \mathrm{Kmol}^{-1}$ is expected. The increase observed in the NMR experiment is more narrow. As the frequency shift of the NMR signals carries the greatest weight 


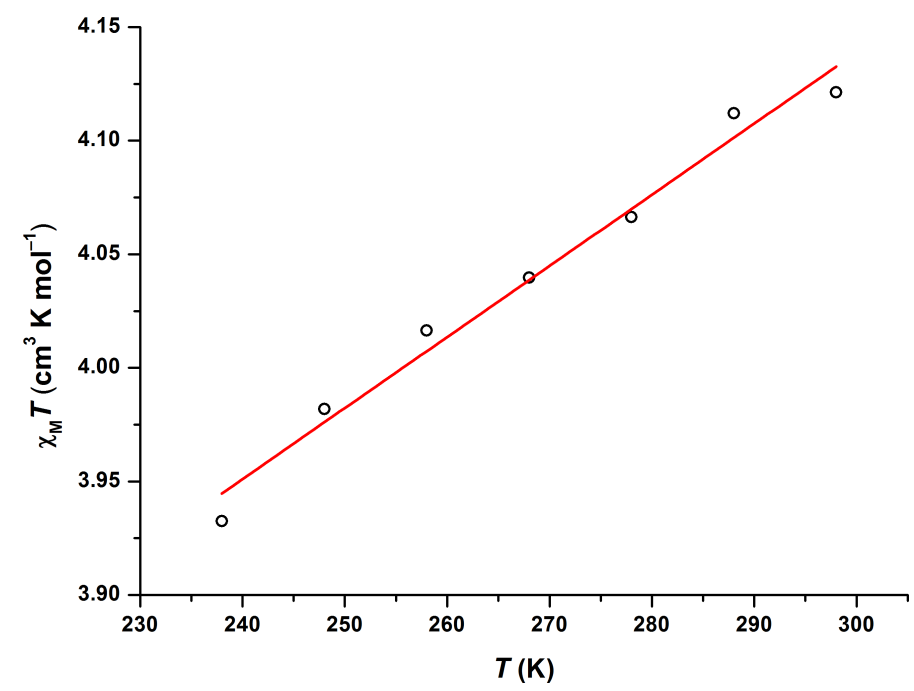

Figure 4.16: $\chi_{\mathrm{M}} T$ vs. $T$ plot for NMR data of $\mathbf{1 a}$.

in these calculations, errors and deviations most likely result from the NMR experiment itself. It is possible that waiting times with lowering the temperature were too short. Consequently, it is possible that inner and outer tube did not maintain the same temperature which would effect the measurement immensely. Also 1a is highly sensitive to dioxygen especially in solution. Over the course of the experiment it is possible that small portions of the sample were oxidized as sample and coaxial tube were mainly sealed with caps and parafilm, which allow for slow oxygen diffusion.

\subsection{Synthesis and Structural Characterization of Diferrous Complexes with $\mathrm{HL}^{\mathrm{Py}}$}

In this section diferrous complexes with the pyridine based bis(pentadentate) ligand system $\mathbf{H L}^{\mathbf{P y}}$, initially developed by SANDER for ruthenium-based bimetallic water oxidation catalysts, are investigated. 120 In analogy to the previously discussed diferrous complexes iron salts with $\mathrm{OTf}^{-}$and $\mathrm{BF}_{4}^{-}$counterions were used for complexation. Moreover a detailed spectroscopic analysis is presented for the complexes in solid state and solution.

\section{$\left[\mathrm{L}^{\mathrm{Py}}\{\mathrm{Fe}(\mathrm{MeCN})\}_{2}\right](\mathrm{OTf})_{3}, 0.5 \mathrm{H}_{2} \mathrm{O}(2 \mathrm{a})$}

Synthesis and Crystallographic Structure

The synthesis of $\mathbf{2 a}$ is based on a similar approach as for $\mathbf{1 a}$ and $\mathbf{1 b}$. In a glovebox the ligand precursor is deprotonated with the base $\mathrm{NaO} t \mathrm{Bu}$ and subsequently reacted with the iron salt $\mathrm{Fe}(\mathrm{OTf})_{2} \cdot 2 \mathrm{MeCN}$. As the ligand is hygroscopic and dry solvents may contain traces of water it proved useful to stir the ligand precursor in MeCN over activated $3 \AA$ molecular sieves for $1 \mathrm{~d}$ prior to complexation. Dark red single crystals were obtained by diethyl ether diffusion into the reaction mixture.

2a crystallizes in the triclinic space group $P \overline{1}$ with one complex cation and three OTf ${ }^{-}$ counterions as well as 0.5 water molecules in the unit cell. The complex cation is three- 


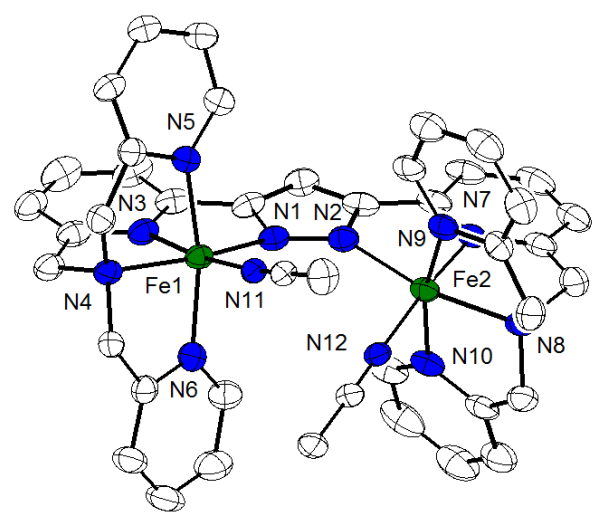

Figure 4.17: Crystal structure of the cation of $\left[\mathrm{L}^{\mathrm{Py}}\{\mathrm{Fe}(\mathrm{MeCN})\}_{2}\right](\mathrm{OTf})_{3}, 0.5 \mathrm{H}_{2} \mathrm{O}$ 2a. Thermal ellipsoids are set to $50 \%$. Hydrogen atoms, solvent molecules as well as the triflate anions were omitted for clarity. Selected bond lengths $[\AA]$ and angles $\left[^{\circ}\right]$ Fe1-N1 2.006(4), Fe1-N3 1.897(4), Fe1-N4 1.999(4), Fe1-N5 1.997(4), Fe1-N6 1.970(4), Fe1-N11 1.938(4), Fe2-N2 2.000(4), Fe2-N7 1.906(4), Fe2-N8 2.000(4), Fe2-N9 1.966(4), Fe2-N10 1.983(4), Fe2-N12 1.925(4), Fe1-N11-C40 173.5(4), Fe2-N12-C42 176.4(4), Fe1-N1-N2-Fe2 30.871(7).

fold positively charged. The coordination sphere of each iron atom can be described as pseudo-octahedral with six nitrogen donor atoms. Five donor sites are provided by the pentadentate chelating ligand system and one donor site is added by the coligand acetonitrile. The monoanionic pyrazolate backbone together with the threefold positively charged cationic structure leaves an oxidation state of $+\mathrm{II}$ for both iron sites. Figure 4.17 depicts the cation of $\mathbf{2 a}$.

Compared to the diiron sites of $\mathbf{1 a}$ and $\mathbf{1 b}$ the bridging pyrazolate and the two iron centers in $\mathbf{2 a}$ do not lie in a plane. The torsion angle $\mathrm{Fe}-\mathrm{N}-\mathrm{N}-\mathrm{Fe}$ is almost $30^{\circ}$. The Fe $\cdots$ Fe distance is $4.46 \AA$ and is thus about $0.2-0.3 \AA$ larger than in complexes with $\mathbf{H L}^{\mathbf{I m}}$. The binding pocket of the cation features two MeCN coligands in a torsion angle of $50^{\circ}$. Both coligands coordinate in a close to linear fashion to the metal ion $\left(173.5^{\circ}\right.$ and $\left.176.4^{\circ}\right)$. Average $\mathrm{Fe}-\mathrm{N}$ bond lengths range between 1.9 to $2.0 \AA$, which is indicative for $\mathrm{Fe}(\mathrm{II})$ low spin complexes. 140 .

\section{$\left[\mathrm{L}^{\mathrm{Py}}\{\mathrm{Fe}(\mathrm{MeCN})\}_{2}\right]\left(\mathrm{BF}_{4}\right)_{3}(\mathbf{2 b})$}

\section{Synthesis and Crystallographic Structure}

Single crystals of $\mathbf{2} \mathbf{b}$ were obtained via the same synthesis route described for $\mathbf{2 a}$. As iron salt $\left[\mathrm{Fe}(\mathrm{MeCN})_{6}\right]\left(\mathrm{BF}_{4}\right)_{2}$ was used for the complexation in the presence of a base in MeCN. Crystals of $\mathbf{2 b}$ feature a dark red colour. $\mathbf{2} \mathbf{b}$ crystallizes in the monoclinic space group $P 2_{1} / \mathrm{n}$ with one complex cation and three $\mathrm{BF}_{4}^{-}$anions in one asymmetric unit. One of the $\mathrm{BF}_{4}^{-}$anions was found to be disordered over one position in a 0.71/0.29 ratio. Both iron sites are pseudo-octahedrally coodinated by six nitrogen donors as in $\mathbf{2 a}$, five of which are provided by the ligand and one added by the MeCN coligand. The cation of $\mathbf{2} \mathbf{b}$ is threefold positively charged. Considering the monoanionic character of the pyrazolate an oxidation state of $+\mathrm{II}$ for both iron atoms results. The bridging pyrazolate and the two iron centers feature a torsion $\mathrm{Fe}-\mathrm{N}-\mathrm{N}-\mathrm{Fe}$ of about $30^{\circ}$ and the $\mathrm{Fe} \cdots \mathrm{Fe}$ distance is $4.46 \AA$. 
Figure 4.18 shows a representation of the cation of $\mathbf{2} \mathbf{b}$.

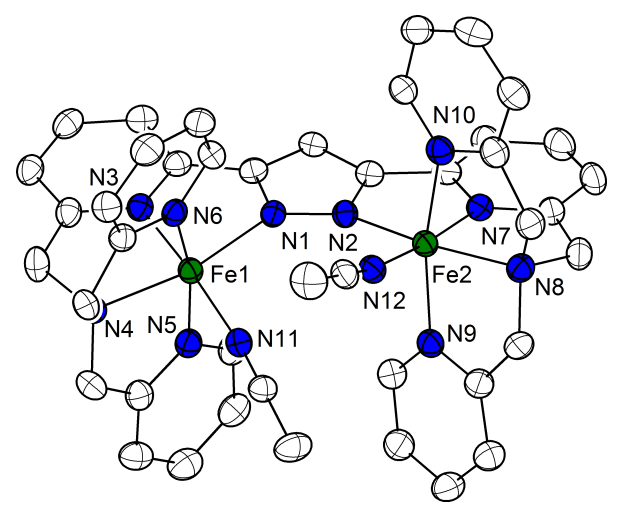

Figure 4.18: Crystal structure of the cation of $\left[\mathrm{L}^{\mathrm{Py}}\{\mathrm{Fe}(\mathrm{MeCN})\}_{2}\right]\left(\mathrm{BF}_{4}\right)_{3}$ 2b. Thermal ellipsoids are set to $50 \%$. Hydrogen atoms as well as the $\mathrm{BF}_{4}^{-}$anions were omitted for clarity. Selected bond lengths $[\AA]$ and angles $\left[{ }^{\circ}\right] \mathrm{Fe} 1-\mathrm{N} 12.003(3), \mathrm{Fe} 1-\mathrm{N} 3$ 1.900(3), Fe1-N4 2.009(3), Fe1-N5 1.988(3), Fe1-N6 1.969(3), Fe1-N11 1.942(3), Fe2-N2 2.024(3), Fe2-N7 1.899(3), Fe2-N8 2.001(3), Fe2N9 1.975(3), Fe2-N10 1.995(3), Fe2-N12 1.954(3), Fe1-N12-C40 174.0(3), Fe2-N11-C42 169.5(3), $\mathrm{Fe} 1-\mathrm{N} 2-\mathrm{N} 1-\mathrm{Fe} 2-29.289(5)$.

The torsion of the two MeCN coligands with respect to the iron-pyrazolate plane is close to $45^{\circ}$. The angle of one nitrile coligand coordinated to the metal is $174^{\circ}$, which is in agreement with the angles found for $\mathbf{2 a}$. The second nitrile coordinates in a slightly more tilted fashion $\left(169^{\circ}\right)$. Fe-N distances lie in the range of 1.9 to $2.0 \AA$, suggesting an overall low spin configuration for the diiron core. Table 4.4 represents selected bond lengths and angles for $\mathbf{2 a}$ and $\mathbf{2} \mathbf{b}$.

Table 4.4: Comparison of selected bond lengths and angles in $\mathbf{2 a}$ and $\mathbf{2} \mathbf{b}$.

\begin{tabular}{clcc}
\hline & Bond length $[\AA]$ & $\mathbf{2 a}$ & $\mathbf{2 b}$ \\
\hline $\mathrm{Fe} 1$ & $\mathrm{Fe}-\mathrm{N}^{\mathrm{Pz}}$ & 2.006 & 2.003 \\
$\mathrm{Fe} 1$ & $\mathrm{Fe}-\mathrm{N}^{\mathrm{MeCN}}$ & 1.938 & 1.942 \\
$\mathrm{Fe} 2$ & $\mathrm{Fe}-\mathrm{N}^{\mathrm{Pz}}$ & 2.000 & 2.024 \\
$\mathrm{Fe} 2$ & $\mathrm{Fe}-\mathrm{N}^{\mathrm{MeCN}}$ & 1.925 & 1.954 \\
\hline & Bond angle $\left[^{\circ}\right]$ & $\mathbf{2 a}$ & $\mathbf{2 b}$ \\
\hline & $\mathrm{Fe} 1-\mathrm{N} 1-\mathrm{N} 2-\mathrm{Fe} 2$ & 30.871 & -29.289 \\
& $\mathrm{Fe} 1-\mathrm{N}-\mathrm{C}^{\mathrm{MeCN}}$ & 173.5 & 176.4 \\
& $\mathrm{Fe} 2-\mathrm{N}-\mathrm{C}^{\mathrm{MeCN}}$ & 174.0 & 169.5 \\
\hline
\end{tabular}

\subsubsection{ESI Mass Spectrometry}

ESI mass spectrometry was performed to verify the composition of the obtained complexes $\mathbf{2 a}$ and $\mathbf{2} \mathbf{b}$ and to check for possible fragmentation under ionizing conditions. The mass spectrum for $\mathbf{2 a}$ in MeCN is depicted in Figure 4.19, Several signals for positively charged ions are visible. Interestingly, the complex cation is not fragmented under ESI conditions. Solely the nitrile coligands get lost. The signal for the "naked" threefold positively charged complex cation at $m / z=251$ is very weak. The main species observed in the spectrum, 
are twofold positively charged. Especially monoanionic ligands from all kinds of sample residues can coordinate to the highly positively initially threefold charged cation to yield the twofold positively charged species.

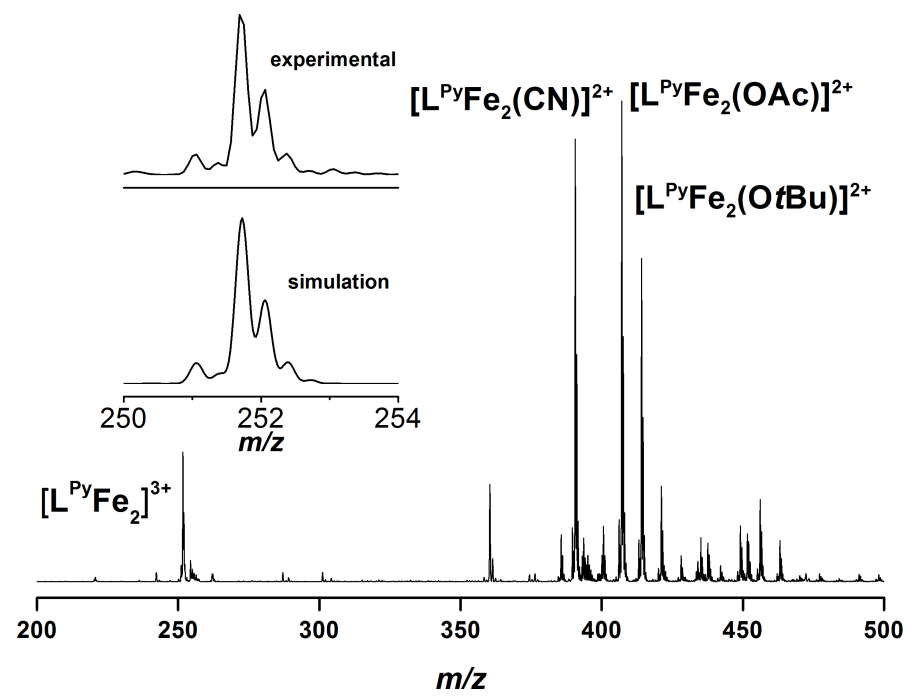

Figure 4.19: $\mathrm{ESI}^{+}$mass spectrum of $\mathbf{2 a}$ in MeCN. Simulation of the signal at $m / z=251$ of the threefold positively charged cation without nitrile coligands.

For $\mathbf{2} \mathbf{b}$ very similar signals were observed. For details see Appendix 11.2 .2

\subsubsection{Mössbauer Spectroscopy and SQUID Magnetometry}

Fe-N bond lengths from the crystal structures of $\mathbf{2 a}$ and $\mathbf{2} \mathbf{b}$ suggested an overall Fe(II) low spin state for both atoms of the diiron core. To verify these findings, Mössbauer spectra and SQUID signatures of crystalline material were recorded. The respective Mössbauer spectra are depicted in Figure 4.20 .
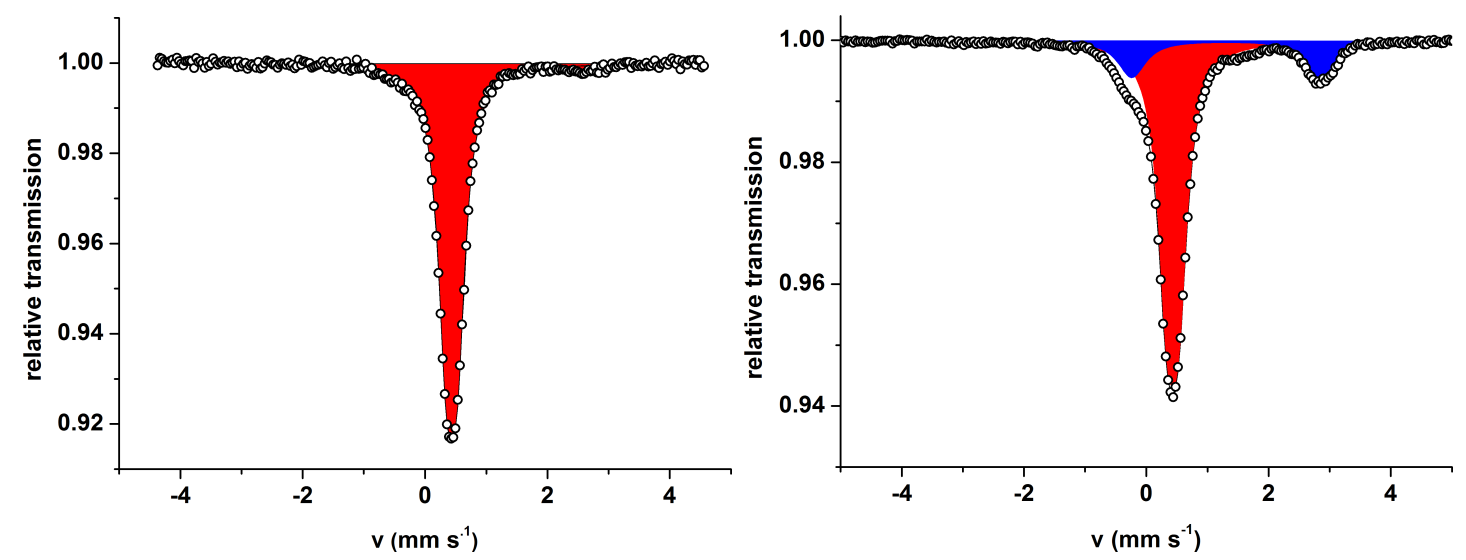

Figure 4.20: Mössbauer spectra of crystalline samples of $\mathbf{2 a}$ and $\mathbf{2} \mathbf{b}$ at $80 \mathrm{~K}$. Isomer shifts $\delta$ and quadrupole splittings $\left|\Delta E_{\mathrm{Q}}\right|$ in $\left[\mathrm{mm} \mathrm{s}^{-1}\right]$. Left: Red subspectrum $\delta=0.43,\left|\Delta E_{\mathrm{Q}}\right|=0.18$. Right: Red subspectrum $\delta=0.43,\left|\Delta E_{\mathrm{Q}}\right|=0.18$, blue subspectrum $\delta=1.30,\left|\Delta E_{\mathrm{Q}}\right|=3.08$. Integral ratio: 80:20.

Both spectra feature one main Mössbauer signal with a very small quadrupole splitting and an isomer shift of approximately $0.43 \mathrm{~mm} \mathrm{~s}^{-1}$ indicative for Fe(II) low spin species. The 


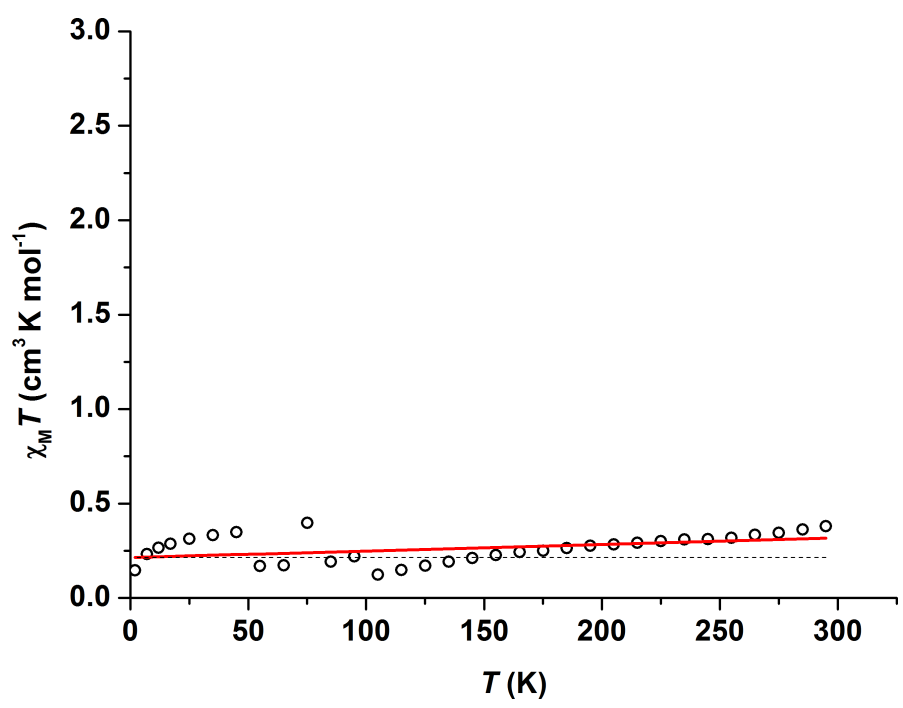

Figure 4.21: $\chi_{\mathrm{M}} T$ vs. $T$ plot for crystalline material of 2a. Fit of the obtained data (red line) and fit of paramagnetic impurity (dashed line).

very narrow splitting parameter may result from a close to octahedral symmetry of the coordination environment and a very symmetrical electron distribution of the $3 \mathrm{~d}$ electrons of the iron atoms. For $\mathbf{2} \mathbf{b}$ a small Mössbauer doublet characteristic for an Fe(II) high spin species, depicted in blue, is present in solid state (20\% area fraction). This doublet can be attributed to either impurities of the initial iron salt from the complexation reaction or to high spin contributions within the sample. Since the binding angle of one MeCN molecule and the anion type differ from $\mathbf{2 a}$ to $\mathbf{2} \mathbf{b}$ it is possible that this factor adds to the high spin contribution in $\mathbf{2 b}$. The bond lengths in solid state crystal structures describe solely an arithmetic mean of the bond lengths found in the crystal. Therefore small high spin contributions are not assignable from x-ray data.

SQUID data were solely obtained for crystalline material of $\mathbf{2 a}$ since $\mathbf{2} \mathbf{b}$ contained variable ratios of high spin contributions in further Mössbauer experiments. Both iron sites displayed a low spin state and $\mathbf{2 a}$ should thus be diamagnetic. $[115]$ The respective $\chi_{\mathrm{M}} T$ vs. $T$ plot is depicted in Figure 4.21

After TIP substraction a $5 \%$ impurity for a spin of $S=5 / 2$ was fit for the data. The solid sample appears to exhibit a low spin character over the entire temperature range. Small inconsistencies between 50 and $100 \mathrm{~K}$ in the spectrum can be attributed to measurement artifacts, which result from a change of the algebraic sign of the magnetization.

\subsubsection{Variable Temperature NMR and UV/Vis Spectroscopy}

Since the diiron core of $\mathbf{2 a}$ features two Fe(II) low spin sites and is diamagnetic, a valuable tool to examine a compound is NMR spectroscopy. The spectrum of $\mathbf{2 a}$ was recorded in $\mathrm{MeCN}_{-} \mathrm{d}_{3}$ (see Appendix 11.2.1 Figure 11.2). At ambient temperature the spectrum features broad signals. Thus parts of the sample exhibit paramagnetic contributions in solution that induce line broadening. Upon cooling to $233 \mathrm{~K}$ the signals sharpen significantly. 
Consequently at ambient temperature high spin states in $\mathbf{2 a}$ are partially populated. This observation was supported by an additional UV/Vis experiment (Appendix 11.2.1 Figure 11.3. In analogy to experiments conducted for $\mathbf{1 a}$ and $\mathbf{1 b}, \mathrm{UV} / \mathrm{Vis}$ bands at $385 \mathrm{~nm}$ of a solution of $\mathbf{2 a}$ in EtCN increase in intensity upon gradual cooling to $193 \mathrm{~K}$ in and decrease upon subsequent warming. This effect is not as pronounced though, as was observed for the SCO in solutions of $\mathbf{1 a}$.

\subsubsection{Nitrile Exchange in Solution}

An experiment to confirm the stability of $\mathbf{2} \mathbf{a}$ in solution and in particular to investigate if the nitrile coligands remain coordinated upon dissolving the sample, is the exchange by a stronger $\pi$ backbonding ligand, such as isonitrile. The binding energies of nitrile and isonitrile in complexes do not differ much. Often though comparing alkylnitriles/alkylisonitriles the isonitrile is favored as coligand to the metal. This behavior is assigned to the stronger $\pi$ backbonding of metals to the isonitrile while the nitrile is mostly a $\sigma$ donor. This trend can easily be reversed when introducing electron withdrawing substituents. $157[158$ ] For the following experiment crystalline material of $\mathbf{2 a}$ was dissolved in dry propionitrile at $-78{ }^{\circ} \mathrm{C}$. $t$-butylisonitrile was added in portions of 0.5 eq. Spectral changes in the UV/Visible range were monitored by using a spectrometer equipped with a dip-probe. The obtained spectra are depicted in Figure 4.22 .

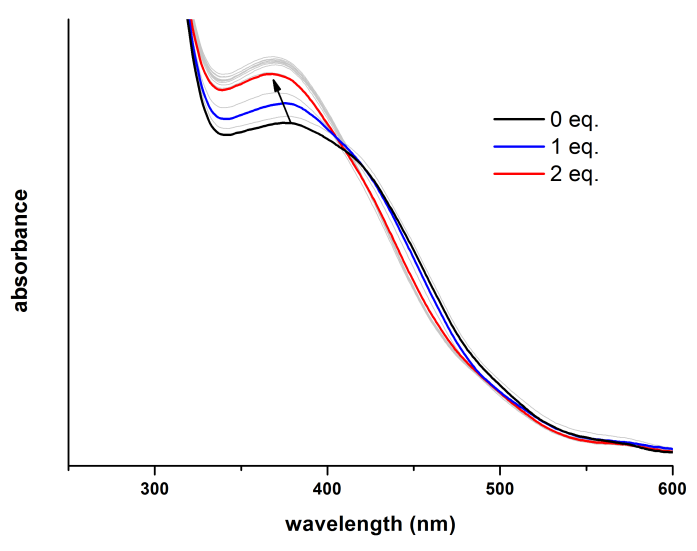

Figure 4.22: Titration of tert-butylisonitrile to a solution of $\mathbf{2 a}$ in EtCN at $-78^{\circ} \mathrm{C}$. Up to 8 eq. of the nitrile were added. After the addition of approximately 2 eq. no further spectral changes were observed.

Approximately 2 eq. of the isonitrile are needed for the exchange reaction. The initial absorption band at $385 \mathrm{~nm}$, which is characteristic for 2a in MeCN and EtCN solutions shifts about $10 \mathrm{~nm}$ to shorter wavelengths and the absorbance increases slightly. It appears that at these low temperatures exactly two isonitrile molecules coordinate as exogenous coligands to $\mathbf{2 a}$ and possibly exchange previously coordinated solvent ligands. Thus the isonitrile analogue of $\mathbf{2 a}$ exhibits a high stability, particuarly at low temperatures. However, from this titration experiment only it is not possible to assign how many coligands/solvent molecules have been coordinated to $\mathbf{2 a}$ in solution prior to the sequential 
addition of the isonitrile. Also dynamic exchange equilibria between solvent molecules and the added isonitrile cannot be ruled out entirely.

\subsection{Summary and Conclusions}

In summary two new diferrous complexes $\mathbf{1 a}$ and $\mathbf{1} \mathbf{b}$ with the new bis(pentadentate) ligand system $\mathbf{H L}^{\mathbf{I m}}$ and exogeneous acetonitrile coligands have been synthesized and thoroughly characterized. 1a shows spin crossover properties in solid state and solution as demonstrated by structural analysis, SQUID magnetometry, UV/Vis and NMR spectroscopic studies and Mössbauer data.

The ligand scaffold $\mathbf{H L}^{\mathbf{P y}}$ was successfully employed to synthesize two new analogous diferrous complexes $\mathbf{2 a}$ and $\mathbf{2} \mathbf{b}$ with largely low spin character. Aside from a structural analysis of both complexes, 2a was characterized by optical spectroscopy, Mössbauer and SQUID measurements.

In line with other diferrous complexe,s these new examples avoid the use of bridging carboxylates for the stabilization of the diiron core and still impart a high stability with regard to coordination/decoordination of the pyrazolate based ligand scaffold. All of these complexes have to be handled under the exclusion of air and represent therefore suitable candidates to probe the activation of small molecules. Moreover their spectroscopic features are very characteristic and allow for a variety of measurements in this regard. 



\section{Reactivity of the Diiron Core with Dioxygen}

The activation of dioxygen at diiron sites has been probed with a multitude of model systems over the last decades. 6/21/47/114/159] The employment of iron in dinuclear pyrazolate bridged chelating ligands in this context, however, has not been studied until the last couple of years. Two main ligand scaffolds have been developed in the MEYER group for this purpose. In a first system by BURGER, the diiron site is embedded in a chelating complex with bis(methylimidazolylmethyl)amine side arms, which provide an $\left\{\mathrm{N}_{4}\right\}$ donor set for each metal center (Scheme 5.1). 126. An additional bridging carboxylate was found to serve as coligand for stabilization. This complex readily reacts with dioxygen to yield an "oxo-dimer" as product. Attempts to elucidate the oxygenation reaction mechanistically based on structural considerations and stopped-flow UV/Vis spectra indicated the formation of a tetranuclear peroxo intermediate by reaction of two complex cations with one dioxygen molecule. Further characterization, though, was prevented due to the thermal sensitivity and short-lived nature of the intermediate.

In case of a second system developed by KindERMAnN (Scheme 5.1) with pyrazolate/tacn hybrid ligands, dioxygen activation clearly proceeded via the formation of a spectroscopically traceable intermediate at low temperatures. ${ }^{128}$ In this ligand scaffold the diiron site is symmetrically coordinated by two $\left\{\mathrm{N}_{4} \mathrm{O}\right\}$ donor sets provided by the chelating nitrogen based ligand and a carboxylate coligand. From symmetry considerations and comparison with spectroscopic signatures of model systems in literature two different intermediates seemed to be feasible. One option involved the formation of a $\mu$-1,1-diiron(III) peroxide. The other possibility was a symmetric superoxide species.

In order to get further insight into possible mechanisms of $\mathrm{O}_{2}$-activation at diiron sites, the previously described diferrous precursors $\mathbf{1 a}, \mathbf{1} \mathbf{b}$ and $\mathbf{2 a}$ are employed to study their behavior in dioxygen activation and oxygenation chemistry by means of various spectroscopic methods in this chapter. They differ from the previous obtained pyrazolate based model systems as they do not contain a bridging carboxylate ligand and also feature longer $\mathrm{Fe} \cdots \mathrm{Fe}$ distances. The additional pyridine functionalities in the pyrazolate backbone moreover might contribute to providing a different electronic structure and thus different reactivity. As many diferrous complexes, the developed compounds are also very sensitive towards dioxygen and intermediates readily decompose. This is why samples for reactivity studies of these oxidation experiments were handled at low temperatures, posing high challenges to the isolation of solid and pure materials. 


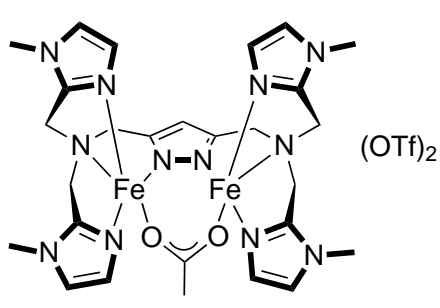

XV
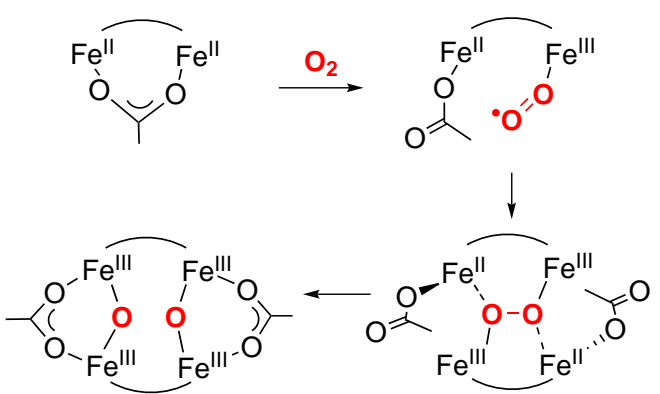

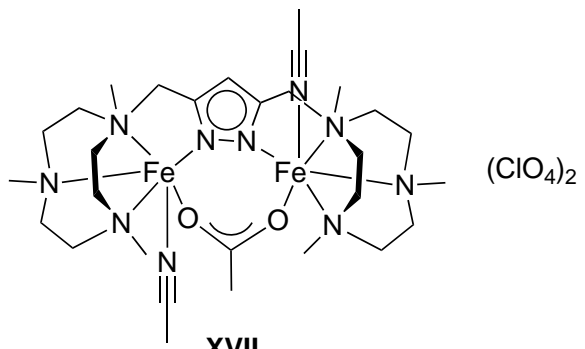

XVII

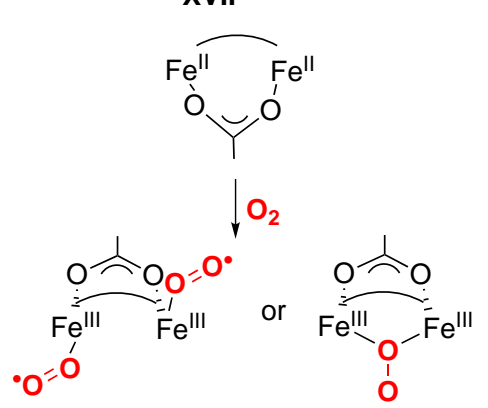

Scheme 5.1: $\mathrm{O}_{2}$ activation at pyrazolate based model systems developed by BURGER und KINDERMANN.

\subsection{Dioxygen Activation with the Diferrous Complex 1.}

As described in Chapter 4, the diferrous complex 1a features two distinct iron sites, where the five-coordinate iron atom displays a high spin ground state and the six-coordinate atom a low spin state. When diluted solutions of $\mathbf{1 a}$ are exposed to air, a color change from orange to red occurs. Hence an oxidation reaction is feasible. In the following section spectroscopic methods are applied to further elucidate these oxidation processes.

\subsubsection{Oxidation in Air}

When a methanol solution of $\mathbf{1 a}$ is exposed to air, the initially orange solution turns dark red within seconds to minutes. This is in agreement with findings for many ferrous solutions at ambient conditions. After many attempts to isolate the oxidation product of 1a, the slow diffusion of diethyl ether into the reaction solution succeeded in the growths of small single crystals in the course of weeks. These single crystals exhibited a dark red color and proved to be very hygroscopic. Standard techniques for x-ray diffraction in which the single crystal is mounted in the x-ray beam in a frozen oil did not apply as the crystals decomposed within seconds in all mineral oils. It was possible, though, to transfer a crystal directly from the mother liquor onto the diffractometer. The obtained molecular structure (3) is depicted in Figure 5.1. 3 crystallizes in the triclinic space group $P \overline{1}$ with one complex cation, four triflates, one diethyl ether, and three methanol molecules in the unit cell. The two iron sites in the cation of $\mathbf{3}$ are not equally coordinated. Both iron atoms are bridged by the pyrazolate of the ligand backbone and additionally by a hydroxo ligand. One iron atom is coordinated by seven donors in a $\left\{\mathrm{N}_{5} \mathrm{O}_{2}\right\}$ donor environment. One coordination site is occupied by a water molecule, which as well as the hydroxo ligand may stem from dioxygen, moisture in the air or residual water in the solvent. This sevenfold coordination geometry is not very common for diiron complexes but has been 
observed before for complexes with this particular ligand. 132] The second iron atom is sixfold coordinated by the nitrogen donors of the ligand backbone and the hydroxo ligand. The complex cation of $\mathbf{3}$ is fourfold positively charged. Consequently, as the pyrazolate and the hydroxo bridge are monoanionic ligands, a +III charge results for both iron sites. The Fe $\cdots \mathrm{Fe}$ distance is $3.75 \AA$, which is significantly shorter than in the diferrous precursor 1a $(4.22 \AA)$. Fe-N bond lengths vary between 2.0 and $2.5 \AA$. Especially the bonds Fe1-N4 and Fe2-N10 were found to be exceptionally long. The ferric diiron core in $\mathbf{3}$ is contracted compared to its ferrous precursor, that is enforced by the hydroxo bridge, which might account for these long $\mathrm{Fe}-\mathrm{N}$ distances.

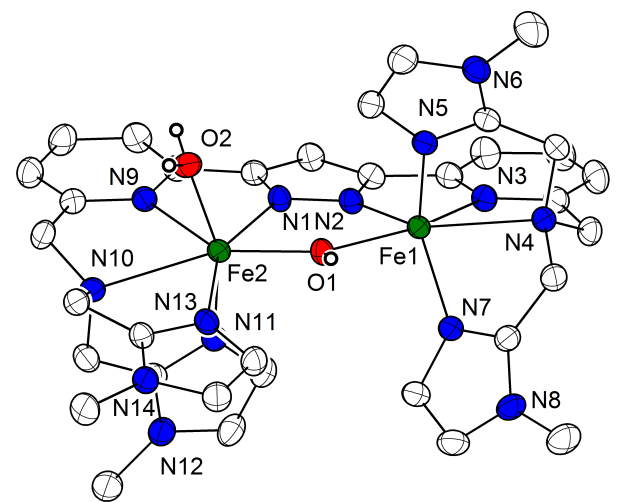

Figure 5.1: Molecular structure of the cationic portion of $\left[\mathrm{L}^{\mathrm{Im}} \mathrm{Fe}\left\{\mathrm{Fe}\left(\mathrm{H}_{2} \mathrm{O}\right)\right\}(\mu-\mathrm{OH})\right]\left(\mathrm{SO}_{3} \mathrm{CF}_{3}\right)_{4}$, $\mathrm{Et}_{2} \mathrm{O}, 3 \mathrm{MeOH}$ 3. Thermal ellipsoids are set to $50 \%$. Most hydrogen atoms as well as the triflate anions and solvent molecules were omitted for clarity. Selected bond lengths $[\AA]$ and angles $\left.{ }^{\circ}\right]$ Fe1-O1 2.009(2), Fe1-N2 2.013(3), Fe1-N3 2.217(3), Fe1-N4 2.421(3), Fe1-N5 2.009(2), Fe1-N7 2.017(3), Fe2-O1 2.038(2), Fe2-O2 2.010(2), Fe2-N1 2.125(3), Fe2-N9 2.373(3), Fe1-N10 2.469(3), Fe2-N11 2.059(2), Fe2-N13 2.158(3), Fe1-O1-Fe2 136.26(13), Fe1-N2-N1-Fe2 -19.478(3).

Especially the rather long $\mathrm{Fe}$...Fe distance might provide an explanation for the observed difficulties to isolate the initial oxidation product of $\mathbf{1 a}$ with molecular oxygen. One of the most common motifs, which is formed upon oxygenation of a diiron core at higher temperatures, is a $\mu$-oxo diiron complex. In the present case, however, the stiff ligand scaffold does only allow for intermetallic distances and $\mathrm{Fe}-\mathrm{O}-\mathrm{Fe}$ bonds, which are way above the limit for such an oxo bridge. 160 Instead, a hydroxo bridge was found, which allows for longer $\mathrm{Fe}-\mathrm{O}$ bond lengths. Most likely this is a result of consecutive reactions after the actual oxygenation event (e.g. by ligand exchange or protonation of the oxo bridge). These consecutive reactions might give different products and account for the difficulties in isolating oxygenation products of 1a. It might furthermore explain the fast decomposition of the crystals, when removed from the mother liquor, since even the hydroxo bridge imposes a high strain on the complex core. Despite apparent structural limitations for monoatomic bridges, incorporation of diatomic bridges such as a peroxo ligand seem to be realizable from a ligand design perspective.

The Mössbauer spectrum of $\mathbf{3}$ features two doublets with isomer shifts characteristic for $\mathrm{Fe}(\mathrm{III})$ high spin sites. As the diiron core is not symmetrically coordinated by the ligand backbone and coligands, two distinct subspectra are reasonable. One larger and one smaller quadrupole splitting parameter can be assigned to the sevenfold and sixfold co- 
ordination geometry, respectively. Figure 5.2 displays the recorded zerofield Mössbauer spectrum at $80 \mathrm{~K}$.

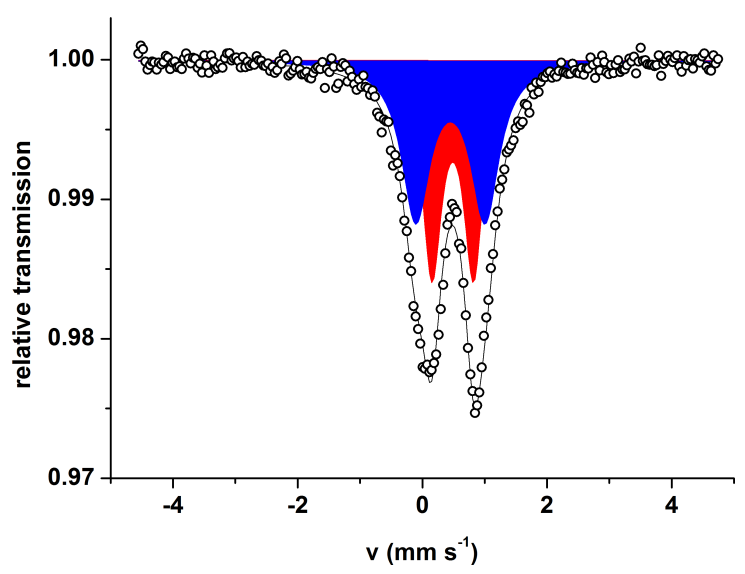

Figure 5.2: Zerofield Mössbauer spectrum of 3 at $80 \mathrm{~K}$. Isomer shifts $\delta$ and quadrupole splittings $\left|\Delta E_{\mathrm{Q}}\right|$ in $\left[\mathrm{mm} \mathrm{s}^{-1}\right]$. Red subspectrum $\delta=0.49,\left|\Delta E_{\mathrm{Q}}\right|=0.67$, blue subspectrum $\delta=0.45$, $\left|\Delta E_{\mathrm{Q}}\right|=1.11$. Signal ratio: $48 \% / 52 \%$.

In order to spectroscopically observe a possible diiron peroxo intermediate and to avoid the subsequent formation of $\mathbf{3}$ due to moisture from air or traces of water in the used solvents, for all subsequent reactions dry solvents were used. Furthermore, pre-dried dioxygen (from a lecture bottle, passed over a column of $\mathrm{P}_{4} \mathrm{O}_{10}$ ) was used to avoid traces of moisture and to ensure that dioxygen was the actual oxidizing agent.

\subsubsection{Reactivity with Dry Dioxygen}

The reaction of $\mathbf{1 a}$ or $\mathbf{1} \mathbf{b}$ (or shortly termed $\mathbf{1}$ in the following) with dry dioxygen was followed by UV/Vis spectroscopy. As oxo, peroxo and hydroxo model complexes in literature exhibit prominent spectroscopic signatures, these characteristics might help identifying intermediates in the reaction of 1 with dioxygen. Figure 5.3 depicts a recorded UV/Vis spectrum of 1a upon the addition of dioxygen in dry EtCN.

The black line represents the typical spectrum of low spin 1a at low temperatures in EtCN as described in Chapter 4 Section 4.3.2. The addition of dioxygen at $-78^{\circ} \mathrm{C}$ yields a putative intermediate with very weak absorption patterns and no characteristic maxima (orange line). Allowing this mixture to warm to ambient temperature leads to the appearance of a shoulder at 360-370 nm, which should account for an oxidized diiron(III) species (red line). However, the strong absorption band at $417 \mathrm{~nm}$ in EtCN somewhat masks bands that might arise from the coordination of dioxygen. A solubility assay was performed and similar spectra were recorded in DCM and methanol as representatives for an apolar aprotic and a polar protic solvent. In these two solvents at low temperatures the absorption band at $417 \mathrm{~nm}$ for $\mathbf{1 a}$ and also the $\mathrm{BF}_{4}^{-}$analogue $\mathbf{1} \mathbf{b}$ is not visible.

As described in detail in Chapter 4 the absorption band at $417 \mathrm{~nm}$ in nitriles can be associated with a spin crossover process from high spin to low spin with decreasing temperature. However, SCO behavior has only been observed exclusively in nitrile solutions for 1a. In methanol or DCM the spin state of $\mathbf{1 a}$ and $\mathbf{1 b}$ is not known.

When reacting a sample of $\mathbf{1 b}$ in DCM with dioxygen at $-70^{\circ} \mathrm{C}$ and slowly warming to 

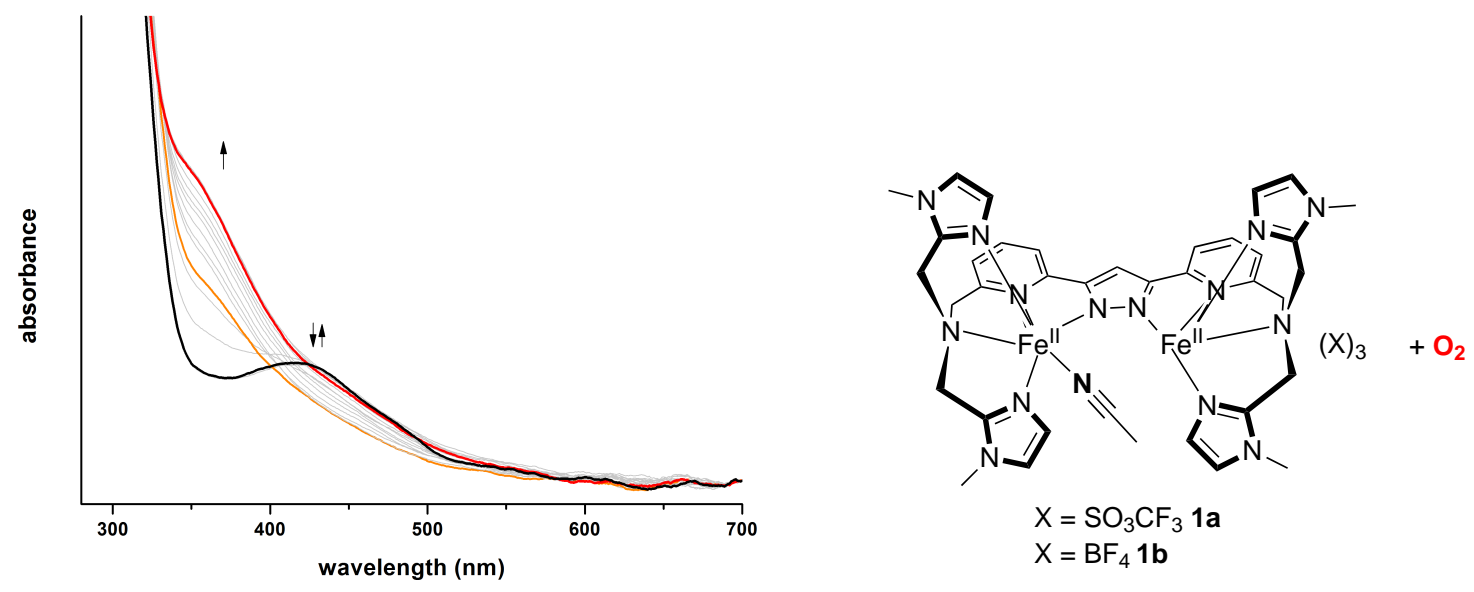

Figure 5.3: UV/Vis spectrum of $1 \mathrm{a}$ in $\mathrm{EtCN}$ at $-78^{\circ} \mathrm{C}$ (black line). Formation of an oxygenated product after addition of $\mathrm{O}_{2}$ and warming to ambient temperature (red line). The orange line indicates a possible diiron oxygen intermediate spectrum at $-78^{\circ} \mathrm{C}$ with negligible spectroscopic signatures. The signal-to-noise ratio in all spectra was smoothed by a Savitzky-Golay fit. The arrows indicate the change of a respective band upon the addition of dioxygen at $-78^{\circ} \mathrm{C}$ and subsequent warming to ambient temperature.

$-8^{\circ} \mathrm{C}$ weak shoulders at $438 \mathrm{~nm}$ and approximately $360 \mathrm{~nm}$ appear (red line). At $-70^{\circ} \mathrm{C}$ without subsequent warming the spectrum of displays a stable but featureless putative intermediate (orange line). In order to isolate the putative intermediate $\mathbf{1} \mathbf{b}^{\mathbf{I n t}}$, cold hexane was added to a solution of $\mathbf{1 b}$ at $-70^{\circ} \mathrm{C}$ to yield an orange precipitate. To record a Mössbauer spectrum of $\mathbf{1} \mathbf{b}^{\text {Int }}$, the solid was carefully transferred into a Mössbauer sample holder on a block of dry ice. The powder sample was flashfrozen and measured. Figure 5.4 depicts the described UV/Vis spectrum of $\mathbf{1 b}$ and the Mössbauer spectrum of the putative intermediate $\mathbf{1 b}^{\text {Int }}$ isolated from a DCM solution.
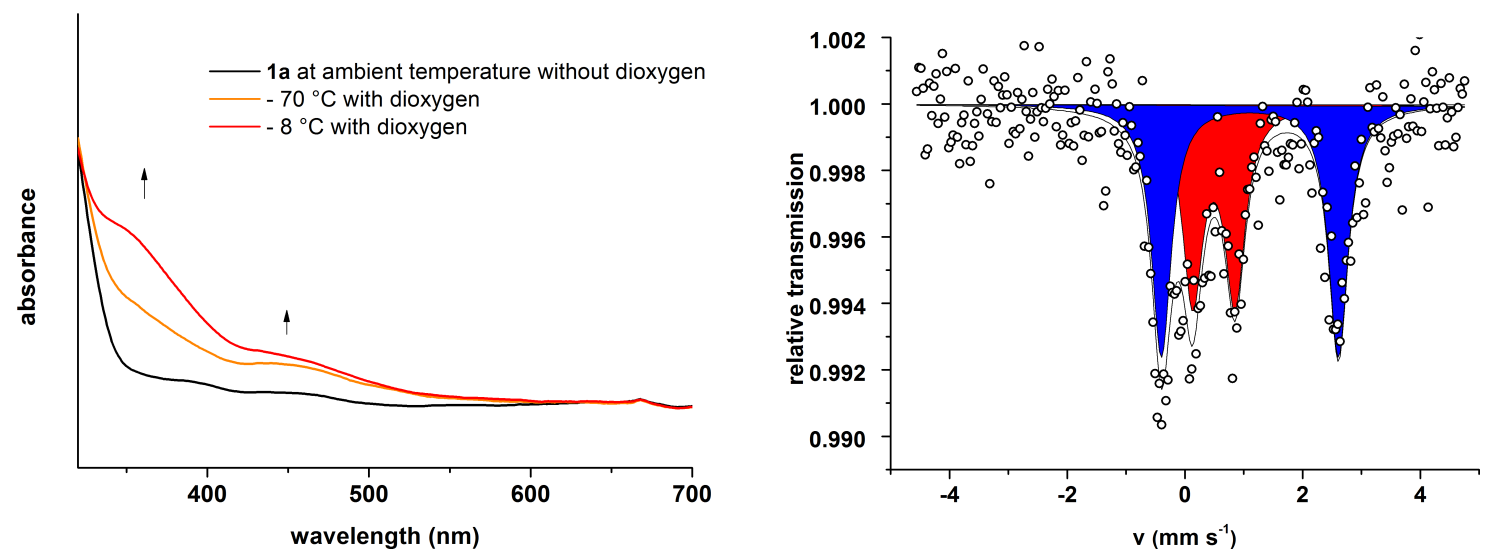

Figure 5.4: Left: UV/Vis spectrum of $\mathbf{1 b}$ in DCM. The initial spectrum at ambient temperature is represented by the black line, putative intermediate at $-70^{\circ} \mathrm{C}$ (orange line) and final oxygenated product (red line). Right: Zerofield Mössbauer spectrum of $\mathbf{1} \mathbf{b}^{\text {Int }}$ at $80 \mathrm{~K}$. Isomer shifts $\delta$ and quadrupole splittings $\left|\Delta E_{\mathrm{Q}}\right|$ in $\left[\mathrm{mm} \mathrm{s}^{-1}\right]$. Red subspectrum $\delta=0.49,\left|\Delta E_{\mathrm{Q}}\right|=1.10$, blue subspectrum $\delta=0.72,\left|\Delta E_{\mathrm{Q}}\right|=3.01$. Signal ratio: $44 \% / 56 \%$.

The Mössbauer spectrum of $\mathbf{1} \mathbf{b}^{\text {Int }}$ isolated at low temperatures exhibits two signals in an almost 1:1 ratio. The blue doublet with large quadrupole splitting and an isomer shift of $0.72 \mathrm{~mm} \mathrm{~s}^{-1}$ can most likely be assigned to a Fe(II) species in a high spin state. The assign- 
ment of the red doublet, however, is less trivial. From the isomer shift a ferric species can be assumed. The prediction of possible binding modes of dioxygen though in accordance with the UV/Vis data at hand is challenging. In comparison with the Mössbauer spectrum of $\mathbf{3}$, it becomes apparent that the isomer shift of the blue subspectrum in $\mathbf{3}$ matches very well with the ferric species (red subspectrum) found for $\mathbf{1} \mathbf{b}^{\text {Int }}$. Nevertheless, the isolated orange solid was found to be highly temperature sensitive, changing its color to dark red when warmed to room temperature. Thus it is assumed that this solid does not represent the comparatively stable species $\mathbf{3}$ despite partially similar Mössbauer parameters.

Very few examples in literature describe ferric dioxygen intermediates with absorption bands lower than $500 \mathrm{~nm}$. One example represents oxy-hemerythrin with an absorption band at $500 \mathrm{~nm}$ and Mössbauer shifts of $\delta=0.50 \mathrm{~mm} \mathrm{~s}^{-1}\left(\left|\Delta E_{\mathrm{Q}}\right|=2.02 \mathrm{~mm} \mathrm{~s}^{-1}\right)$ and $\delta=0.51 \mathrm{~mm} \mathrm{~s}^{-1}\left(\left|\Delta E_{\mathrm{Q}}\right|=1.01 \mathrm{~mm} \mathrm{~s}^{-1}\right)$. 43 . Recently discussed Fe(III) superoxo intermediates display absorptions below $500 \mathrm{~nm}$. 161 164 However, to date Mössbauer data are hardly available for these intermediates.

Diferric oxo-bridged complexes on the other hand exhibit also characteristic absorption bands in the 300-400 nm region. Mössbauer shifts and quadrupole splittings of the "oxodimer" region match as well with the obtained spectra. 160] Also hydroxo-bridged dimers are known to feature similar UV/Vis and Mössbauer parameters. 160 [165/166] Therefore the obtained spectra cast doubt on whether diferrous $\mathbf{1 b}$ reacts via an intermediate to its oxygenated pendant. The orange solid could just as well represent a mixture of not fully oxygenated $\mathbf{1 b}$ instead of two distinct iron sites within one molecule. On the other hand, the Mössbauer spectrum of $\mathbf{1 b}^{\text {Int }}$ depicts despite the high signal to noise ratio two subspectra with similar integral areas. For a product mixture this would be unlikely.

As a result the following conclusions can be drawn from the obtained spectra: From $\mathrm{UV} / \mathrm{Vis}$ data, the formation of a peroxo intermediate does not seem feasible as most peroxo intermediates exhibit absorption bands above $500 \mathrm{~nm}$. An oxo, hydoxo or superoxo diiron core seems reasonable in this respect. As two Mössbauer doublets with very different parameters were obtained for $\mathbf{1} \mathbf{b}^{\mathbf{I n t}}$, a putative intermediate would not adopt a symmetric geometry with two equal iron sites. From a structural perspective though, an oxo-bridged diiron core seems unlikely as already the hydoxo-bridged diiron site in $\mathbf{3}$ is very unstable and contracted in its coordination geometry. Thus by elimination of possibilities the most likely species observed in the oxidation of $\mathbf{1 b}$ are a superoxo and/or a hydroxo species.

For comparison of the isolated putative intermediate $\mathbf{1} \mathbf{b}^{\text {Int }}$ and the final oxygenation product $\mathbf{1} \mathbf{b}^{\mathbf{O x}}$ a Mössbauer spectrum of $\mathbf{1 b}$ after oxidation and warming up to ambient temperature was recorded. For that purpose a sample of $\mathbf{1 b}$ was dissolved in dry, degassed DCM. Dry dioxygen was added and the mixture was allowed to stirr for a while. Finally the solvent was removed. A powder sample of $\mathbf{1 b}^{\mathbf{O x}}$ was submitted for the measurement. The spectrum is depicted in Figure 5.5 .

Both subspectra of $\mathbf{1} \mathbf{b}^{\mathbf{O x}}$ can be assigned to Fe(III) species. Isomer shifts and quadrupole splittings differ slightly from the parameters found for $\mathbf{1} \mathbf{b}^{\text {Int }}$ and $\mathbf{3}$. The red subspectrum in $\mathbf{1} \mathbf{b}^{\mathbf{O x}}$ could be comparable to the red subspectrum in $\mathbf{1 b}^{\mathbf{I n t}}$ and the blue subspectrum in 


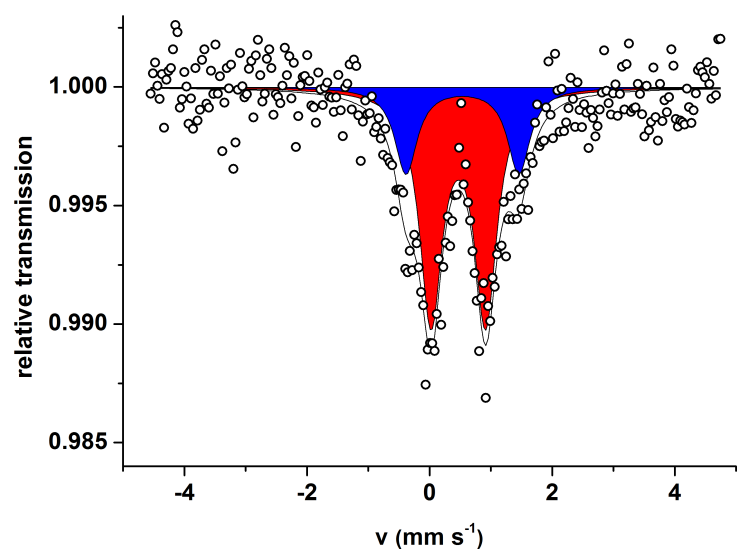

Figure 5.5: Mössbauer spectrum of $\mathbf{1} \mathbf{b}^{\mathbf{O} x}$ at $80 \mathrm{~K}$. Isomer shifts $\delta$ and quadrupole splittings $\left|\Delta E_{\mathrm{Q}}\right|$ in $\left[\mathrm{mm} \mathrm{s}^{-1}\right]$. Red subspectrum $\delta=0.47\left|\Delta E_{\mathrm{Q}}\right|=0.89$, blue subspectrum $\delta=0.54\left|\Delta E_{\mathrm{Q}}\right|=1.84$. Signal ratio: $73 \% / 27 \%$.

3, but since also in this Mössbauer spectrum the signal to moise ratio is very high a direct comparison is purely speculative. Evaluating the obtained spectra it seems reasonable to assume that an oxo and/or even more likely a hydroxo-bridged diiron complex as found for 3 forms upon oxygenation. As Mössbauer shifts of peroxo, oxo and hydroxo bridged complexes are often very similar from these parameters alone it is impossible to assign structural features for the final oxygenation product $\mathbf{1} \mathbf{b}^{\mathbf{O x}}$. Table 5.1 summarizes all Mössbauer parameters.

Table 5.1: Comparison of Mössbauer parameters for putative oxygenation products and intermediates.

\begin{tabular}{c|lll}
\hline & $\mathbf{1 b}^{\text {Int }}$ & $\mathbf{1 b}^{\mathbf{O x}}$ & $\mathbf{3}$ \\
\hline$\delta\left[\mathrm{mm} \mathrm{s}^{-1}\right]$ & $0.49,0.72$ & $0.47,0.54$ & $0.49,0.45$ \\
$\left|\Delta E_{\mathrm{Q}}\right|\left[\mathrm{mm} \mathrm{s}^{-1}\right]$ & $1.10,3.01$ & $0.89,1.84$ & $0.67,1.11$ \\
\hline
\end{tabular}

Another widely established method to gain insight into the nature of diiron oxygen intermediates and thus reaction pathways is resonance Raman spectroscopy as peroxo and superoxo $\mathrm{O}-\mathrm{O}$ bonds as well as $\mathrm{Fe}-\mathrm{O}$ bonds feature characteristic vibrational modes. However, attempts to perform these experiments with $\mathbf{1} \mathbf{b}^{\text {Int }}$ failed due to the fast decomposition of $\mathbf{1} \mathbf{b}^{\text {Int }}$ and overlaps with solvent bands in solution spectra. Mass spectra of the oxidized material $\mathbf{1} \mathbf{b}^{\mathrm{Ox}}$ did not lead to further insights as none of the signals could be assigned properly. Thus in the framework of this work the nature of a putative intermediate and the final oxygenated species $\mathbf{1}^{\mathbf{O} x}$ remains unclear.

Also unknown is the ability of $\mathbf{1}^{\mathbf{O x}}$ to oxygenate substrates at low temperatures. A first attempt was made by adding triphenylphosphine to the putative intermediate $\mathbf{1 b}^{\mathbf{I n t}}$ in DCM at $-40^{\circ} \mathrm{C}$. ESI analysis of the reaction solution revealed the presence of triphenylphosphine oxide as well as triphenylphosphine and the diiron complex. This can be seen as a first indication that $\mathbf{1} \mathbf{b}^{\text {Int }}$ might be capable to oxygenate triphenylphosphine. However, further clarification is needed to provide a more detailed insight into this reaction, e.g., by labeling studies with ${ }^{18} \mathrm{O}_{2}$ and ${ }^{31} \mathrm{P}-\mathrm{NMR}$ analysis of the products. 


\subsection{Dioxygen Activation with the Diferrous Complex 2a.}

Aside from a structural elucidation, the diferrous complex $\mathbf{2 a}$ has been examined spectroscopically as well as by magnetic susceptibility measurements as described in chapter 4. The complex differs from the previously described complexes $\mathbf{1 a}$ and $\mathbf{1} \mathbf{b}$ not only in the ligand scaffold but also electronically as it exhibits a low spin character over a wide temperature range in solid state and solution. Due to the low spin character of $\mathbf{2 a}$, it is expected to react significantly slower with triplet dioxygen than $\mathbf{1 a}$ and $\mathbf{1} \mathbf{b}$ as this process should be spin forbidden.

In the following section UV/Vis and Mössbauer spectroscopy were applied to monitor the complex's behavior upon oxygenation with dioxygen and some other oxygen transfer reagents.

\subsubsection{Reactivity with Dry Dioxygen}

In a first UV/Vis experiment fresh crystals of $\mathbf{2 a}$ were dissolved in dry DCM and dioxygen added at $-65^{\circ} \mathrm{C}$. The choice of the solvent DCM was based on findings from the previous section in which the complex 1a exhibited exclusive UV/Vis bands in nitriles, which masked changes in the investigated spectral range. However, strikingly, the recorded spectrum of $\mathbf{2 a}$ in the non polar aprotic solvent DCM (Figure 5.6 black line) featured very similar absorption bands compared to spectra recorded in nitriles (see Appendix 11.2.1 Figure 11.3). The close similarities of the spectrum of $\mathbf{2 a}$ in DCM with spectra recorded in nitriles especially in the absorption bands at 386 and $415 \mathrm{~nm}$ suggest that 2a features the same structure in both solvents. Due to the fact that the two iron sites in $\mathbf{2 a}$ feature a $\{$ N6 $\}$ coordination sphere in solid state and most likely in nitrile solutions, it could be assumed that the exogenous coligand MeCN remains bound also in DCM solutions, which imposes a high mole fraction of molecules in the low spin state. Also the compared to 1 significantly slower full oxygenation over the course of a few hours at $-35^{\circ} \mathrm{C}$ might be explained by a predominantly low spin character of $\mathbf{2} \mathbf{a}$ and by coordinative saturation of bot iron ions.

The strong absorption of low spin $\mathbf{2 a}$ in the 350 to $450 \mathrm{~nm}$ region, however, poses difficulties on following UV/Vis absorption bands that may be due to the oxygenation reaction. The only apparent observation that can be made is a decrease of the absorption bands at 386 and $415 \mathrm{~nm}$ upon the addition of dioxygen. Moreover the oxygenation reaction is significantly slower compared to solutions of $\mathbf{1 a}$ and $\mathbf{1 b}$.

As the low spin character of $\mathbf{2 a}$, which may be explained by the $\left\{\mathrm{N}_{6}\right\}$ coordination environment of each iron center, might be responsible for the sluggish reactivity towards dioxyen, attempts were made to partially change the spin states of the iron centers by removing the MeCN coligands. This should also diminish the strong absorption bands at $386 \mathrm{~nm}$ and $415 \mathrm{~nm}$, making identification of possible intermediates in the reaction with dioxygen easier.

Therefore crystals of $\mathbf{2 a}$ were dried for several days and mildly heated. No apparent color change of the crystalline material occurred. Upon dissolving the dried material in DCM, however, it became apparent that the strong absorption bands in the 350 to $450 \mathrm{~nm}$ region featured significantly lower intensities. Thus presumably a higher mole fraction 

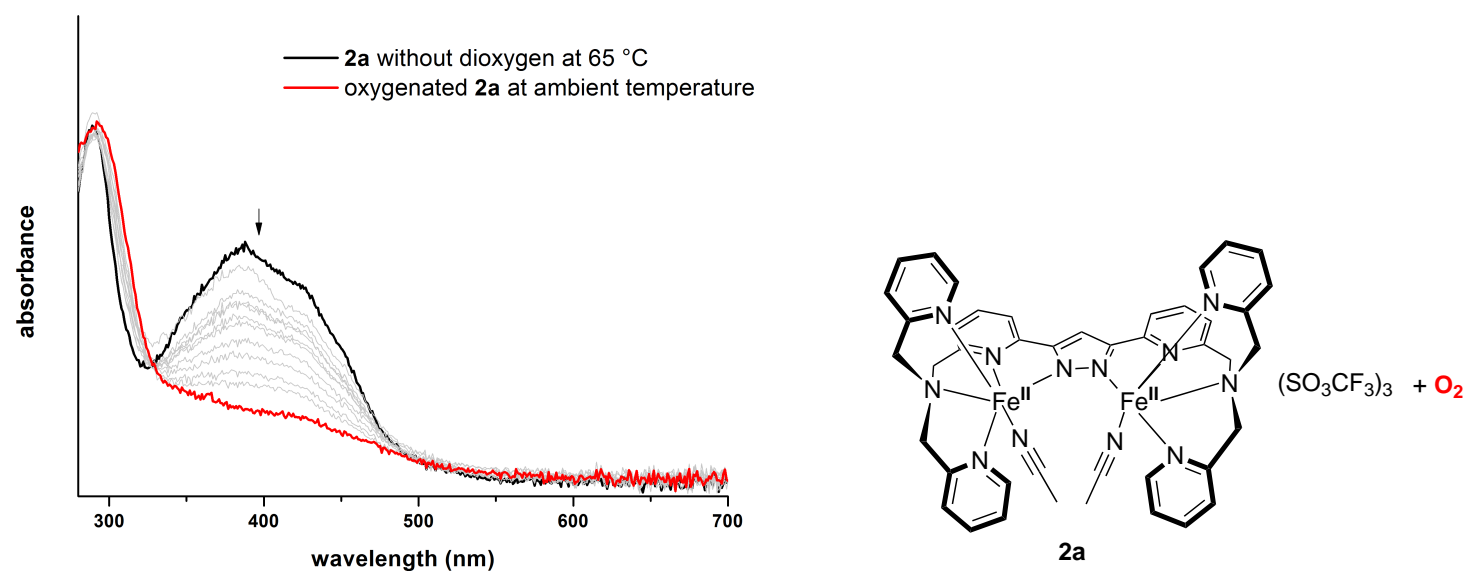

Figure 5.6: UV/Vis spectrum of $\mathbf{2 a}$ in $\mathrm{DCM}$ at $-65^{\circ} \mathrm{C}$ (black line). Addition of $\mathrm{O}_{2}$ at $-65^{\circ} \mathrm{C}$ and subsequent warming to ambient temperature. The intensity of the absorption band at $415 \mathrm{~nm}$ starts to decrease at temperatures around $-35^{\circ}$. The red line indicates the absorption spectrum of the final oxidized species.

of molecules featured a high spin state after drying. The recorded spectrum is shown in Figure 5.7.

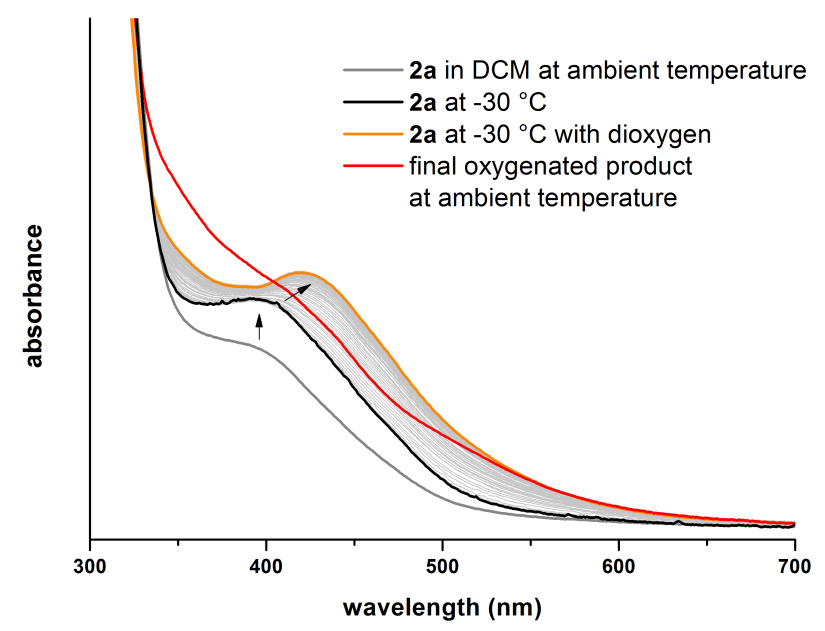

Figure 5.7: UV/Vis spectrum of dried 2a in DCM. The grey line indicates the spectrum of $\mathbf{2 a}$ at ambient temperature. Upon cooling to $-30{ }^{\circ} \mathrm{C}$ the intensity of this band increases (black line). The addition of dry dioxygen induces a shift of the absorption maximum to $422 \mathrm{~nm}$ (orange line). The red line resembles fully oxygenated $\mathbf{2 a}$ at ambient temperature.

$\mathrm{O}_{2}$ was added to a solution of dried $\mathbf{2 a}$ in $\mathrm{DCM}$ at $-30{ }^{\circ} \mathrm{C}$. The initial band at $390 \mathrm{~nm}$ shifts to $422 \mathrm{~nm}$ upon oxygenation (orange line) within $1 \mathrm{~h}$. This species, however, is not characteristic for the final oxygenated product (red line) and may account for an intermediate. Attempts to isolate this intermediate as a frozen solution or powder sample for Mössbauer measurements were not successful, though.

Thus it remains unclear if the oxygenation of $\mathbf{2 a}$ proceeds via the formation of an intermediate. A comparison of the absorption band at $422 \mathrm{~nm}$ with metric parameters of literature 
known model systems for peroxo complexes shows that an absorption in this range would be unusually blue shifted. 21 However, absorption bands in the range of $300-400 \mathrm{~nm}$ were observed for pyrazolate based diiron complexes before. Kindermann reported the formation of an intermediate when exposing a pyrazolate bridged diiron(II) precursor to $\mathrm{O}_{2}$ at $-40{ }^{\circ} \mathrm{C}$. The formed species featured absorption bands at 310 and $385 \mathrm{~nm}$ and decayed upon warming to yield an oxo bridged diferric product. 128. The absorption bands of the intermediate were attributed to either a $\mu$-1,1-peroxo or a superoxo species.

Since apart from UV/Vis data no further spectral or structural information are available for $\mathbf{2} \mathbf{a}^{\mathbf{O x}}$, no further assignment can be made to elucidate the low temperature activation of dioxygen. Signals in ESI mass spectra did not lead to a conclusive assignment of any species.

\subsubsection{Reactivity with Oxygen Transfer Reagents}

To probe whether the reactivity and UV/Vis spectra of 2a differed with the employment of oxygen transfer reagents instead of dioxygen, respective measurements were performed. The recorded UV/Vis spectra are shown in Figure 5.8.
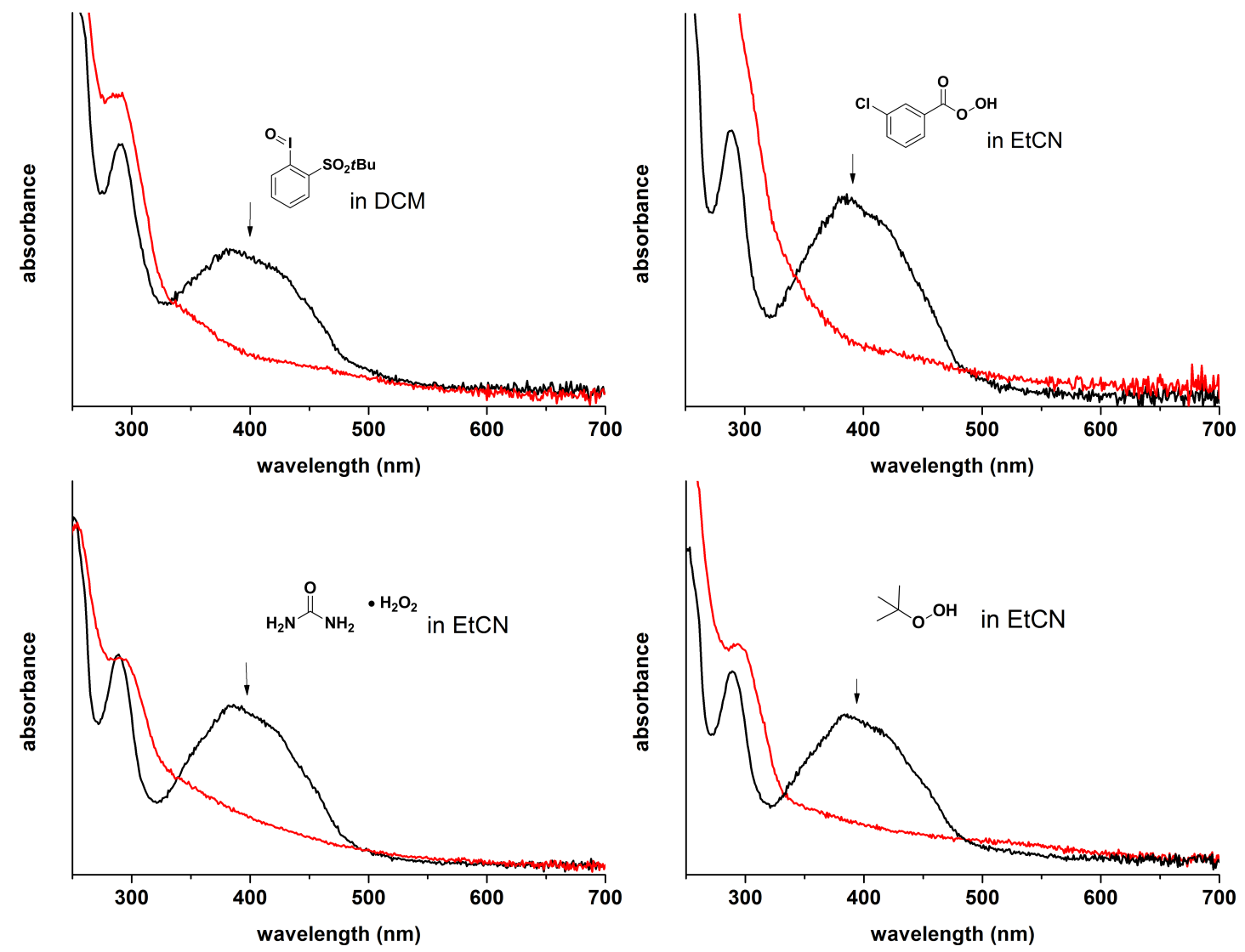

Figure 5.8: Oxygenation of 2a with different oxidants (excess). Top left: soluble iodosobenzene, top right: metachloroperbenzoic acid, bottom left: urea hydrogenperoxide adduct, bottom right: tertbutylhydroperoxide.

All spectra were recorded at temperatures around $-70^{\circ} \mathrm{C}$. The black line in all spectra represents the signature of the starting material $\mathbf{2 a}$ dissolved in the respective solvent 
presumably with largely low spin character. The red line represents the final spectrum of the oxygenated product. The spectra do not differ very much from the spectrum of $\mathbf{2 a}$ upon with the addition of dioxygen (Figure 5.6). Thus for all oxygenation reactions a common product $\mathbf{2} \mathbf{a}^{\mathbf{O x}}$ can be expected.

\subsection{Summary and Conclusions}

In this chapter the reactivity of $\mathbf{1} \mathbf{a} / \mathbf{1} \mathbf{b}$ and $\mathbf{2 a}$ with dioxygen was probed. The activation of dioxygen by the respective diiron precursor was primarily followed by UV/Vis and if possible by Mössbauer experiments.

Due to the high thermal sensitivity of the primary products of $\mathbf{1 a}, \mathbf{1} \mathbf{b}$ and $\mathbf{2} \mathbf{a}$ upon oxygenation, it was often not possible to obtain more spectroscopic data on the nature of these potential intermediates. Raman studies, sophisticated cryo-mass experiments as well as NMR measurements might help further to elucidate these oxygenation reactions. However, attempts to obtain resonance Raman spectra of the intermediate did not only fail due to challenges in isolating a suitable sample, but probably also since the wavelengths of the laser excitation $(457 \mathrm{~nm}$ and $633 \mathrm{~nm}$ ) did not match the observed UV/Vis absorptions The oxidation of 1a with dioxygen in the air revealed that moisture and water traces in solvents may lead to a faster decay of putative diiron oxygen intermediates. The x-ray structure and Mössbauer spectrum of $\mathbf{3}$ unambiguously substantiate this fact.

The use of several oxygen transfer reagents for the oxygenation of $\mathbf{2 a}$ did not provide spectral information that significantly differed from the use of plain dioxygen. Also high valent species were not observed in these experiments.

The oxygenation of substrates is yet to be thorougly tested. First attempts seemed promising but lack a more elaborate analysis by labeling and NMR experiments. However, the nature of putative diiron oxygen intermediates and whether they exhibit electrophilic or nucleophilic reactivity is unknown. Hence, extensive substrate screening did not seem reasonable. Overall it can be concluded that the complexes $\mathbf{1 a}, \mathbf{1} \mathbf{b}$ and $\mathbf{2 a}$ react with dioxygen and are oxidized even at low temperatures. A diiron peroxo species was spectroscopically not observed. Structurally the formation of diiron oxo species seemed unlikely so that upon oxygenation possibly several species were formed which could decompose in subsequent reactions. Therefore the analysis of intermediates and products was practically not possible. 



\section{Diiron Dinitrosyl Complexes and their Reactivity upon Reduction}

As described in the introductory sections, Flavodiiron proteins have gained increasing interest in research over the last decades. ${ }^{9196}$ Expressed by some microbes against "nitrosative stress", these enzymes are capable to catalyze the two electron reduction of NO to the less toxic $\mathrm{N}_{2} \mathrm{O}$. So far almost exclusively spectroscopic data are available for the characterization and mechanistic understanding of these enzymes which makes the synthesis and use of low molecular weight analogues for comparison inevitably important. The previously described model systems by the LEHNERT and the LIPPARD/MOËNnELoCCOZ-group are currently the only diiron-dinitrosyl systems that have shown to produce a significant amount of $\mathrm{N}_{2} \mathrm{O}$ under reductive conditions or exposure to light. 102[103] The reactive pathway for the liberation of $\mathrm{N}_{2} \mathrm{O}$ of these systems on the other hand is under intense discussion. Particularly the biological motif of nitric oxide binding in Flavodiiron enzymes as well as in respective model systems is of great interest. Pyrazolate based diferrous complexes have been shown to react with dioxygen quite rapidly and are thus expected to also readily bind nitric oxide.

The reactivity of nitric oxide with diferrous complexes containing pyrazolate/tacn hybrid ligands has already been investigated in the MEYER group. ${ }^{128}$ Therein new $\left[\{\mathrm{Fe}(\mathrm{NO})\}^{7}\right]_{2}$ complexes were synthesized and structurally as well as spectroscopically characterized. Interestingly, nitric oxide was found to bind in an anti-orientation in these complexes, which led to a new scope of reactivity. When reducing these $\left[\{\mathrm{Fe}(\mathrm{NO})\}^{7}\right]_{2}$ complexes with cobaltocene the $\left[\{\mathrm{Fe}(\mathrm{NO})\}^{9}\right]_{2}$ pendant as well as a $\mathrm{Fe}(\mathrm{II}) \mathrm{Fe}(\mathrm{II})$ species in a $1: 1$ ratio were obtained.
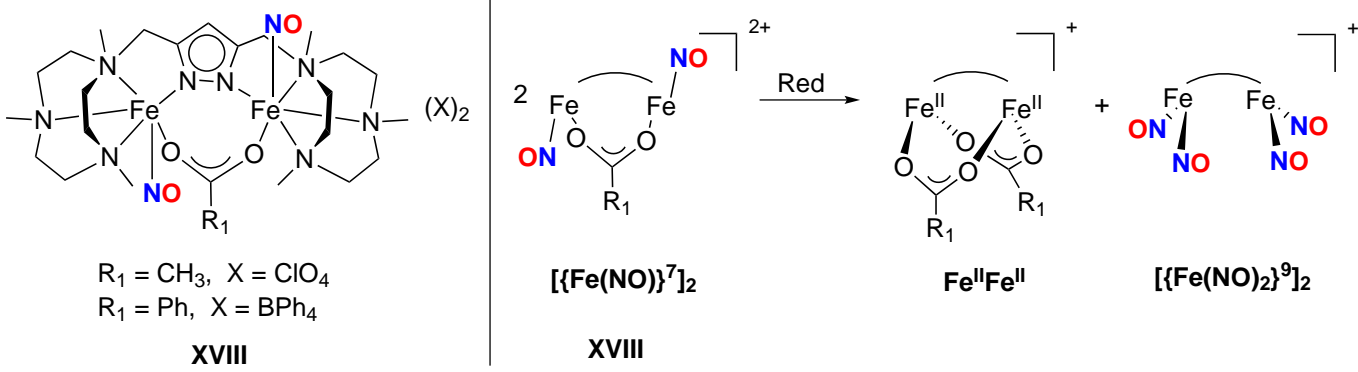

$\mathrm{Fe}^{\text {II }} \mathrm{Fe}$

$\left[\left\{\mathrm{Fe}(\mathrm{NO})_{2}\right\}^{9}\right]_{2}$

Scheme 6.1: $\left[\{\mathrm{Fe}(\mathrm{NO})\}^{7}\right]_{2}$ complexes with pyrazolate/tacn-hybrid ligands and their reactivity. 
In this chapter the activation of nitric oxide with the previously in Chapter 4 presented diferrous precursors $\mathbf{1 a}$ and $\mathbf{2 a}$ is probed along with reactivity studies upon reduction. The exogeneous acetonitrile coligands should easily substitute for nitric oxide and thus result in the formation of the respective $\left[\{\mathrm{Fe}(\mathrm{NO})\}^{7}\right]_{2}$ species. This chapter comprises the structural and spectrocopic characterization of these newly formed diiron dinitrosyl complexes as well as first reactivity studies mainly followed by spectroscopic techniques such as IR- and Mössbauer spectroscopy.

\subsection{Preparation and Structural Characterization of $\left[\{\mathrm{Fe}(\mathrm{NO})\}^{7}\right]_{2}$ Complexes}

The preparation of the dinitrosyl analogues of the in Chapter 4 presented diiron precursors $\mathbf{1 a}$ and $\mathbf{2 a}$ is straightforward. In general, the acetonitrile adduct or diiron precursor is dissolved in an appropiate solvent such as methanol, acetonitrile or propionitrile and the solution is purged with $\mathrm{NO}_{(\mathrm{g})}$ (for the exact setup see the Experimental section). The initially red $\mathrm{Fe}(\mathrm{II})$-solution changes color to brown. In an atmosphere of nitric oxide the $\left[\{\mathrm{Fe}(\mathrm{NO})\}^{7}\right]_{2}$ complexes can be crystallized by diethyl ether diffusion. Since the obtained compounds differ in their spectroscopic and electronic properties they will be discussed separately in the following.

\subsection{1 $\left[\mathrm{L}^{\operatorname{Im}}\{\mathrm{Fe}(\mathrm{NO})\}_{2}\right](\mathrm{OTf})_{3}, \mathrm{MeOH}(4)$}

\section{Synthesis and Crystal Structure}

To obtain the diiron dinitrosyl complex 4, the diiron(II)-precursor 1a was dissolved in methanol in a glovebox. Diethyl ether diffusion into the reaction mixture in an $\mathrm{NO}_{(\mathrm{g})}{ }^{-}$ atmosphere yielded brown single crystals which were suitable for x-ray diffraction. 4 crystallizes in the triclinic space group $P \overline{1}$. The cation is threefold positively charged. Three triflate anions compensate this charge. Additionally a methanol molecule cocrystallizes within the unit cell. Each iron atom is fivefold coordinated by the ligand framework. The sixths ligand is nitric oxide. Consequently, a $\left\{\mathrm{N}_{6}\right\}$-donorset results for each iron site. The nitric oxide ligands are coordinated within the diiron pocket in a tilted syn fashion. The $\mathrm{Fe} \cdots \mathrm{Fe}$ distance is $4.37 \AA$ and the $\mathrm{Fe}-\mathrm{N}(\mathrm{O})$ distances range between $1.78 \AA$ and $1.79 \AA$, respectively. These values are in agreement with distances found in the systems by LEHNERT and LIPPARD/MoËNNE-LoCCOZ. $[81 \mid 102] 103]$ A disorder over two positions was found for the two NO ligands. The occupancy factors for N21-O21 and N22-O22 are 0.96/0.04 and $0.78 / 0.22$, respectively. Due to this disorder a discussion of the $\mathrm{Fe}-\mathrm{N}-\mathrm{O}$ bond angle does not seem adequate as these values range between 125 and $165^{\circ}$. Additionally, all triflate counterions as well as the methanol molecule within the primitive cell were adjusted with a disorder over two positions and, if needed, with EADP constraints. Further selected distances and angles are mentioned in Figure 6.1.

The prediction of the complex's electronic structure from crystallographic data only is challenging. Usually the $\mathrm{Fe}-\mathrm{NO}$ units are best described in the Eneman-Feltham notation 


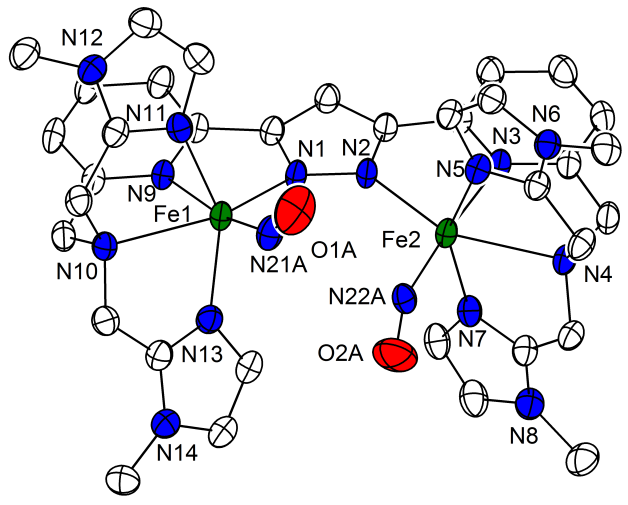

Figure 6.1: Cation of the molecular structure of $\left[\mathrm{L}^{\operatorname{Im}}\{\mathrm{Fe}(\mathrm{NO})\}_{2}\right](\mathrm{OTf})_{3}, \mathrm{MeOH}$ 4. Thermal ellipsoids are set to $50 \%$. Hydrogen atoms as well as the triflate anions and solvent molecules were omitted for clarity. Selected bond lengths $[\AA]$ and angles $\left[{ }^{\circ}\right]$ Fe1-N1 2.071(3), Fe1-N9 2.130(3), Fe1-N10 2.357(3), Fe1-N11 2.101(3), Fe1-N13 2.091(3), Fe1-N21A 1.775(4), Fe1-N21B 1.85(13), Fe2-N2 2.079(3), Fe2-N3 2.122(3), Fe2-N4 2.351(3), Fe2-N5 2.101(3), Fe2-N7 2.107(3), Fe2-N22A 1.788(6), Fe2-N22B 1.70(3), Fe1-N21A-O1A 147.0(5), Fe1-N21B-O1B 125.4(9), Fe2-N22A-O2A 151.6(1), Fe2-N22B-O2B 164.4(5), Fe1-N1-N2-Fe2 23.0(4).

as two distinct $\{\mathrm{Fe}(\mathrm{NO})\}^{7}$ sites.

The metal-ligand distances are very similar for both iron atoms in 4 . In a structural comparison with the model systems XI and XII (see Chapter 1: Introduction) 1 [1]103, 4 features a similar pocket with two $\{\mathrm{Fe}(\mathrm{NO})\}^{7}$ sites coordinated in a syn-fashion. Similar $\mathrm{Fe}-\mathrm{N}$ bond distances compared to these model systems allow for the assumption that the spin for both iron sites in 4 is $S=3 / 2$ and thus high spin.

\section{IR and Mössbauer Spectroscopy}

For a closer insight into the complex's electronic structure infrared spectroscopic data and Mössbauer data were collected and compared to calculated data (ORCA, BP86, def2tzvp, def2-tzvp/j). Figure 6.2 depicts the experimental and calculated IR/ATR spectra of complex 4. Very pronounced in diiron dinitrosyl complexes are bands assigned to the NOstretching region which are highlighted in red. The $\mathrm{N}-\mathrm{O}$ stretch for unbound nitric oxide in the gaseous phase can be found at $1876 \mathrm{~cm}^{-1}$. 167. Two stretching bands were observed for the Fe-NO sites in the experimental spectrum of 4 at $1760 \mathrm{~cm}^{-1}$ and $1733 \mathrm{~cm}^{-1}$. Aside from a slight shift of a few wavenumbers the calculated spectrum matches extremely well with the experimentally obtained data. According to the calculation, the two Fe-NO sites within one molecule couple. The NO ligand, if vibrationally decoupled from the rest of the molecule, can only feature a single stretch as it is a diatomic molecule. However, if in close proximity the NO stretches of the two Fe-NO sites can be either "in phase" or "anti-phase", which is reflected by the two different IR bands in the spectrum.

The theoretical calculation of the IR spectrum of $\mathbf{4}$ was carried out with the original coordinates from the structural analysis. Prior to the calculation these coordinates were not geometry optimized for two reasons. For once it was found that when optimizing 


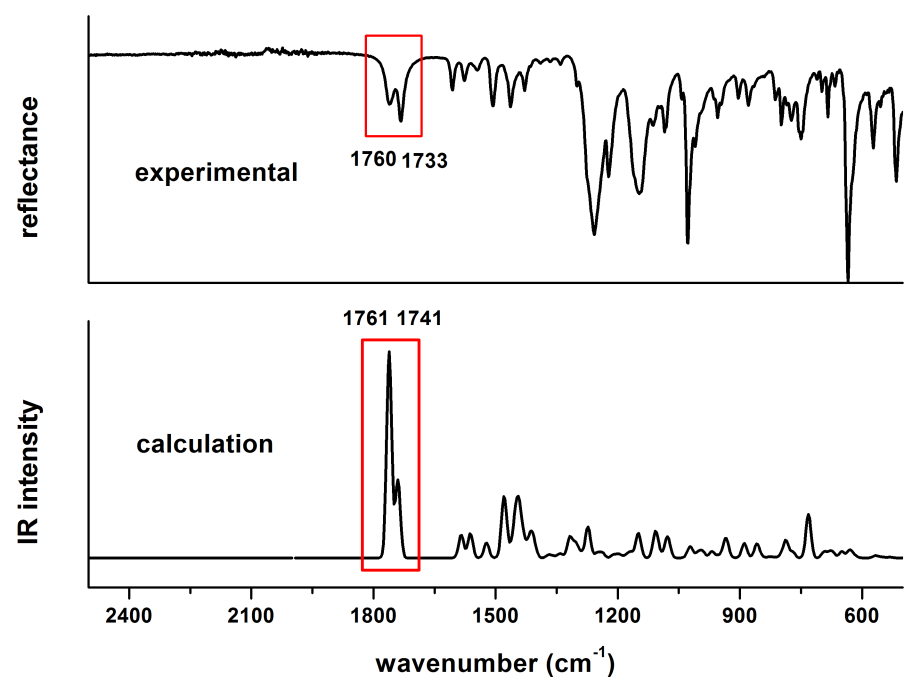

Figure 6.2: Experimental ATR-spectrum of $\left[\mathrm{L}^{\mathrm{Im}}\{\mathrm{Fe}(\mathrm{NO})\}_{2}\right](\mathrm{OTf})_{3}$ and calculated spectrum. The red box highlights the NO-stretching frequencies in the solid state.

the structural coordinates with a def2-tzvp def2-tzvp/j basis set, the two iron centers became equal, which did not reflect an accurate electronic representation of 4 . Secondly experimental IR data were directly obtained from the same batch of crystalline material that was used for the crystal structure determination. The positions of the observed stretching frequencies for $\mathbf{4}$ are moreover in good agreement with the two diiron dinitrosyl complexes in literature $\left(1760 \mathrm{~cm}^{-1}\right.$ (Lehnert) and $1785 \mathrm{~cm}^{-1}$ (Lippard) 81103 In addition, the ${ }^{15} \mathrm{~N}^{18} \mathrm{O}$ labeled pendant of $\mathbf{4}$ was synthesized. Of both complexes (4 and labeled 4) solution IR spectra were recorded in DCM/MeOH 5:1 (Figure 6.3).

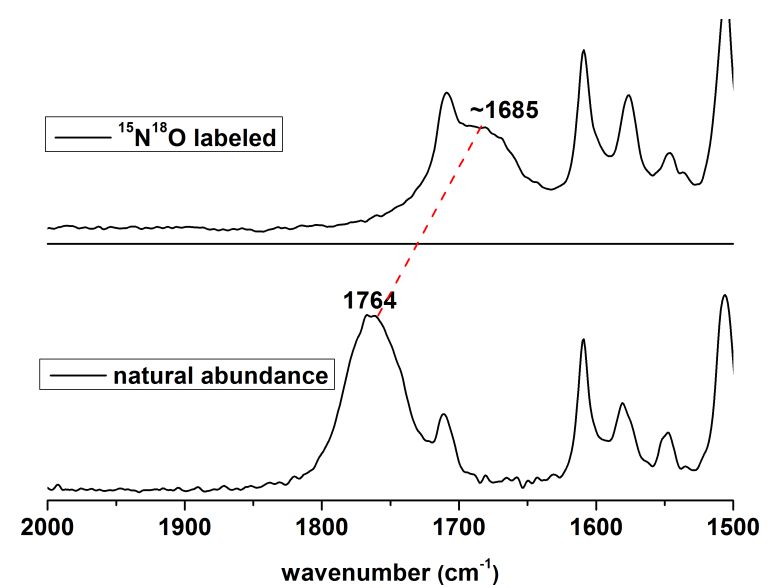

Figure 6.3: Solution IR spectra of 4 and ${ }^{15} \mathrm{~N}^{18} \mathrm{O}$ labeled 4 in DCM/MeOH 5:1.

The nitrosyl stretching band of $\mathbf{4}$ with natural abundant NO appeared in the spectrum at $\tilde{\nu}=1764 \mathrm{~cm}^{-1}$. Upon labeling this band shifted to $1685 \mathrm{~cm}^{-1}$. A shift of approximately $79 \mathrm{~cm}^{-1}$ is in good agreement with labeling studies in literature 103 and confirms the correct assignment of the observed band to the Fe-NO moieties. However, a shift of this magnitude would also be expected for a harmonic oscillation of $\mathrm{N}-\mathrm{O}$ at the observed frequencies. 
Figure 6.4 depicts the zerofield Mössbauer spectrum of 4 at $80 \mathrm{~K}$. Mössbauer data from the same sample batch of $\mathbf{4}$ that was used for the previously described measurements display one main signal, which can be fit with either one or two different subspectra. Since the molecular structure and DFT-calculations suggested that the two metal sites within one complex cation are not fully identical, a representation with two subspectra, but very similar parameters was chosen.

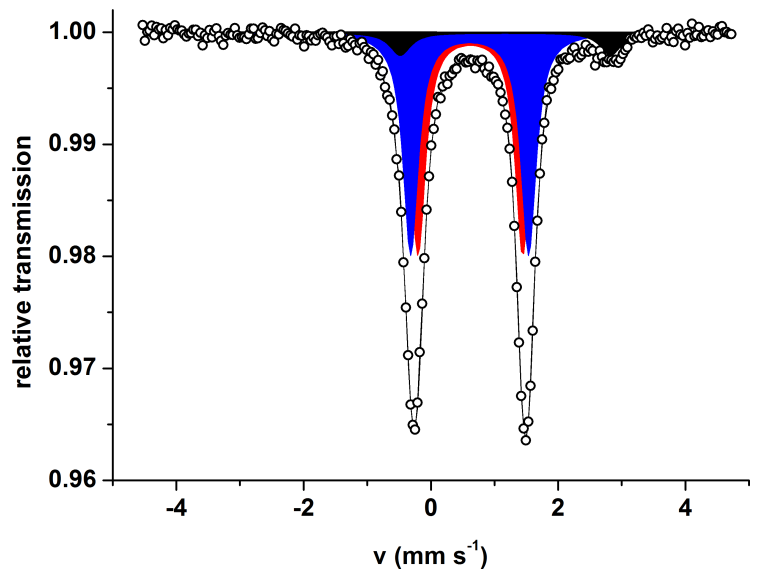

Figure 6.4: Mössbauer spectrum of a crystalline sample of 4 at $80 \mathrm{~K}$. Isomer shifts $\delta$ and quadrupole splittings $\left|\Delta E_{\mathrm{Q}}\right|$ in $\left[\mathrm{mm} \mathrm{s}^{-1}\right]$. Left: Red subspectrum: $\delta=0.62,\left|\Delta E_{\mathrm{Q}}\right|=1.65$, blue subspectrum $\delta=0.61,\left|\Delta E_{\mathrm{Q}}\right|=1.85$. A small impurity of $6.3 \%$, which is most likely an Fe(II)-high spin species is indicated by the black subspectrum.

Moreover, Mössbauer parameters were calculated from the structural coordinates of $\mathbf{4}$ for comparison. The theoretical isomer shifts and quadrupole splittings which can be obtained from DFT-calculations deviate slightly from the experimentally observed. Using a B3LYP $(\mathrm{CP}(\mathrm{PPP}))$ basis set 168 the following parameters were derived using the non optimized coordinates of the crystal structure of $4: \delta=0.49 \mathrm{~mm} \mathrm{~s}^{-1},\left|\Delta E_{\mathrm{Q}}\right|=1.78 \mathrm{~mm} \mathrm{~s}^{-1}$ and $\delta=0.51 \mathrm{~mm} \mathrm{~s}^{-1},\left|\Delta E_{\mathrm{Q}}\right|=1.88 \mathrm{~mm} \mathrm{~s}^{-1}$. The calculated isomer shift deviated from experimental data, since these values were only determined indirectly. The estimated standard deviation for these values is $0.09 \mathrm{~mm} \mathrm{~s}^{-1}$. 168 .

\section{Magnetic Susceptibility Measurements}

To determine the spin states of the two iron sites in $\mathbf{4}$, variable temperature magnetic susceptibility data were measured via SQUID magnetometry. Additionally, Variable Temperature Variable Field (VTVH) measurements at field strengths of $1 \mathrm{~T}, 3 \mathrm{~T}$ and $5 \mathrm{~T}$ were carried out. For the magnetic measurements crystalline material of $\mathbf{4}$ was used. Therefore, inside a glovebox, crystals were placed in a gel capsule and covered with a drop of fluorinated oil to avoid the orientation and movement of the sample when a magnetic field is applied. The capsule was closed with a lid and placed inside a PTFE straw as sample holder. Both measurements are shown in Figure 6.5. 

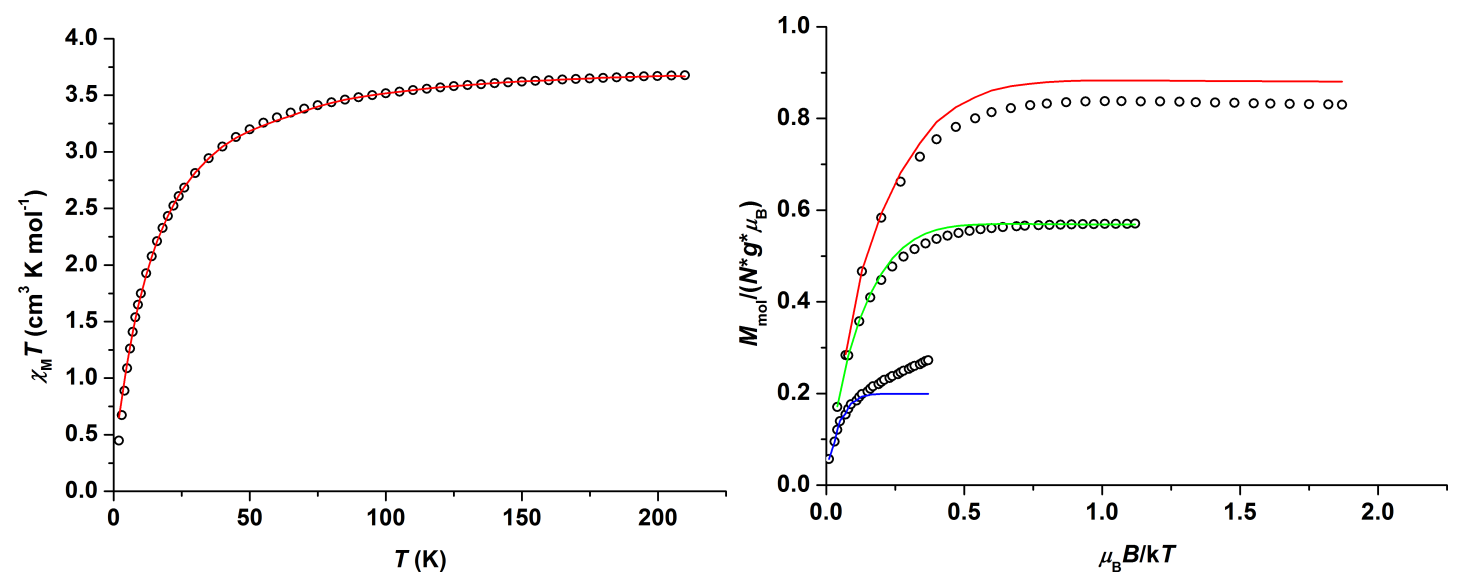

Figure 6.5: Magnetic susceptibility measurement of 4. Left: $\chi_{\mathrm{M}} T$ vs. $T$ measurement indicating a weak antiferromagnetic coupling of two $S=3 / 2$ states. Right: VTVH measurement at field strengths of $1 \mathrm{~T}$ (blue), $3 \mathrm{~T}$ (green) and $5 \mathrm{~T}$ (red). The solid lines represent the best simultaneous fits for both data sets including a DM interaction.

For the simultaneous simulation of all measurements a HeISENBERg DiraC VAN VleCK hamiltonian including the isotropic and anisotropic exchange coupling, zerofield and Zeeman splitting was used. The zerofield splitting parameter $D$, which can be used to explain the decrease of $\chi_{\mathrm{M}} T$ for $T<50 \mathrm{~K}$ was best fitted with a value of $D=-6.85 \mathrm{~cm}^{-1}$. The coupling constant of the two diiron sites was set to $J=-1.98 \mathrm{~cm}^{-1}$. The $g$ values were treated anisotropically with $g_{\mathrm{x}}=g_{\mathrm{y}}=1.94$ and $g_{\mathrm{z}}=2.16$. The best fits for the obtained data included an antisymmetric exchange interaction (Dzyaloshinsky-Moriya interaction, DM). [169[170] Hence the hamiltonian used for the fit contained an additional vector $d$ $\left(d_{\mathrm{Z}}=15.66 \mathrm{~cm}^{-1}\right)$ including this anisotropy. Antisymmetric exchange has been observed in dinuclear complexes before. 171|172] According to MüNCK et al. in diferric systems antisymmetric exchange contains contributions of spin orbit coupling and isotropic exchange. 1721173 All simulations were performed with the program JulX. 174] The measurement was corrected for temperature independent paramagnetism (TIP). $\chi_{\mathrm{M}} T$ vs. $T$ data from SQUID magnetometry confirm two $\left\{\mathrm{Fe}(\mathrm{NO})^{7}\right\}$ sites with $S=3 / 2$ spin states. Spin states of $S=3 / 2$ in iron nitrosyl complexes are often described in literature as $\mathrm{Fe}$ (III) site antiferromagnetically coupled to an $\mathrm{NO}^{-}$ligand. ${ }^{80 \mid 175]}$ However, this picture is simplified as a definite assignment of oxidation states in iron nitrosyl complexes can not be rationalized.

\subsection{2 $\left[\mathrm{L}^{\mathrm{Py}}\{\mathrm{Fe}(\mathrm{NO})\}_{2}\right](\mathrm{OTf})_{3}(5)$}

\section{Synthesis and Crystal Structure}

Complex $\mathbf{5}$ was prepared according to the procedures previously described. Crystalline material that was suitable for x-ray diffraction was obtained by diethyl ether diffusion in an acetonitrile solution of the nitrosylated diiron precursor $\mathbf{2 a}$. The quality of the crystalline material though was very poor and the crystals were often twinned. Unfortunately it was not possible to refine the crystallographic data to a satisfying resolution, so that only a picture can be presented herein. The complex crystallized in the monoclinic space group $P 2_{1} / \mathrm{n}$ with two complex cations and six triflate counterions in the unit cell. The charge 
of each cation is hence +III. Presumably several solvent molecules, specifically acetonitrile and diethyl ether, cocrystallize together with the complex cation of $\mathbf{5}$. Due to the poor quality of the measurement, though, it was not possible to determine the exact nature and stoichiometry of all solvent molecules within the cell. The cation of $\left[\mathrm{L}^{\mathrm{Py}}\{\mathrm{Fe}(\mathrm{NO})\}_{2}\right](\mathrm{OTf})_{3}$ (5) is depicted in Figure 6.6

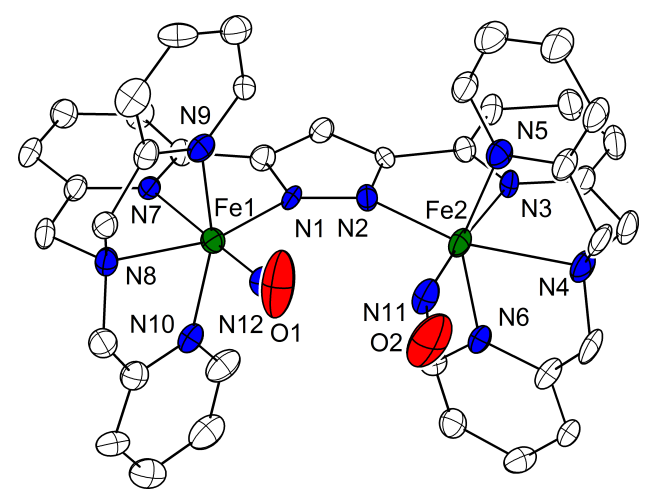

Figure 6.6: Representation of one cation of the molecular structure of $\mathbf{5}$ (picture only). Thermal displacement ellipsoids are set to $30 \%$. Solvent molecules, hydrogen atoms and triflate anions were omitted for clarity. Selected bond lengths $[\AA]$ and angles [ $\left.{ }^{\circ}\right]$ Fe1-N1 2.054(10), Fe1-N7 2.037(9), Fe1-N8 2.107(10), Fe1-N9 2.046(11), Fe1-N10 2.024(11), Fe1-N12 1.710(12), Fe2-N2 2.123(9), Fe2-N3 2.108(11), Fe2-N4 2.264(11), Fe2-N5 2.169(11), Fe2-N6 2.152(11), Fe2-N11 1.728(1), Fe1N12-O1 146.7(14), Fe2-N11-O2 155.1(1), Fe1-N1-N2-Fe2 2.928(2).

The two metal centers in $\mathbf{5}$ lie in an almost perfect plane with the bridging pyrazolate. The torsion angle $\mathrm{Fe}-\mathrm{N}-\mathrm{N}-\mathrm{Fe}$ is $2.93^{\circ}$. Interestingly, the two nitric oxide molecules within the binding pocket are not as strongly tilted as in 4 . They feature a more parallel orientation. The Fe $\cdots$ Fe distance in $\mathbf{5}$ is $4.45 \AA$ and thus slightly larger compared to $\mathbf{4}(4.37 \AA$ ).

Comparing $\mathrm{Fe}-\mathrm{N}$ distances within the cationic portion of $\mathbf{5}$, it can be noted that in average bond lengths associated with Fe1 are $0.1 \AA$ shorter than for Fe2. The electronic structure of the two iron centers cannot be concluded from structural parameters, but it seems likely from the comparison of bond lengths that the two metal sites are not electronically equal.

\section{IR and Mössbauer Spectroscopy}

Infrared spectra were collected of polycrystalline material of $\mathbf{5}$. Figure 6.7 illustrates the experimental as well as the calculated IR/ATR-spectrum. The typical $\mathrm{N}-\mathrm{O}(\tilde{\nu}(\mathrm{N}-\mathrm{O}))$ stretch in both spectra is represented by a broad band with a shoulder, indicating that the Fe-NO moieties in $\mathbf{5}$ couple. From comparison to the calculated spectrum the frequency at higher wavenumbers $\left(\tilde{\nu}(\mathrm{N}-\mathrm{O})=1778 \mathrm{~cm}^{-1}\right)$ can be attributed to an "in-phase" stretching motion whereas the shoulder $\left(\tilde{\nu}(\mathrm{N}-\mathrm{O}) \sim 1750 \mathrm{~cm}^{-1}\right)$ can be assigned to the "anti-phase" stretch. Aside from other stretching motions from the ligand backbone the NO stretch from experimental and calculated data agree within a range of approximately $90 \mathrm{~cm}^{-1}$. In comparison to the experimental ATR-spectrum of $\mathbf{4}$, the frequencies in $\mathbf{5}$ are slightly shifted to higher wavenumbers.

The theoretical calculation of the IR spectrum of $\mathbf{5}$ was carried out with the original coordinates from x-ray data. Prior to the calculation these coordinates were, other than for 4, geometry optimized with a def2-tzvp def2-tzvp/j basis set and a broken symmetry ap- 


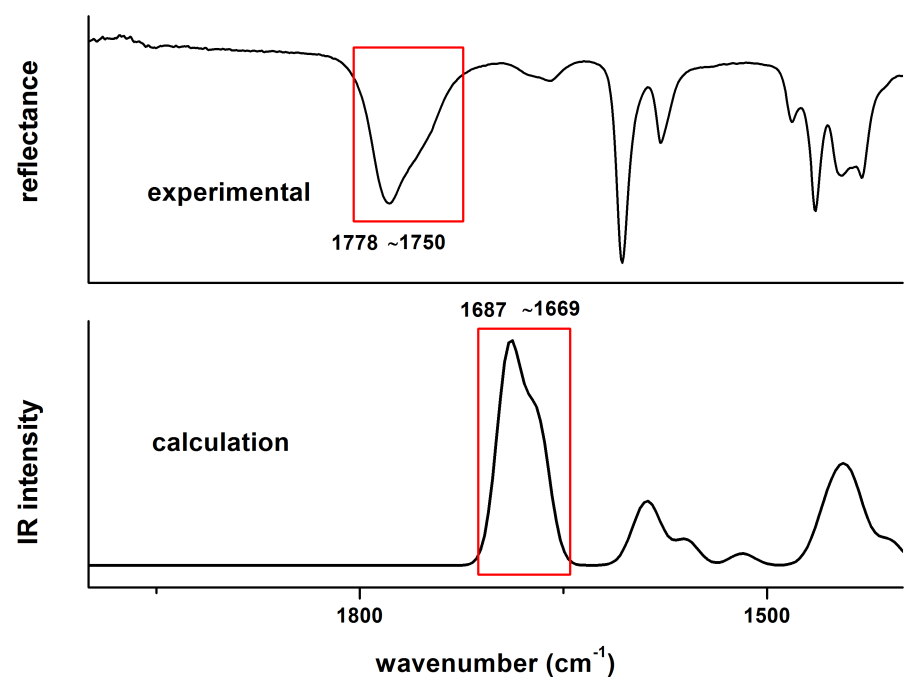

Figure 6.7: Experimental ATR-spectrum and calculated IR spectrum of a polycrystalline sample of 5. The red box highlights the NO-stretching frequencies in the solid state and for the DFT calculations in the gas phase.

proach assuming spin states of $S=3 / 2$ and $S=1 / 2$ for the two Fe atoms in $\mathbf{5}$, respectively. The calculation of a theoretical IR spectrum of $\mathbf{5}$ without geometry optimization led to the generation of negative imaginary frequencies which did not reflect the experimental data.

A more comprehensive insight into the electronic properties of $\mathbf{5}$ was gained by Mössbauer spectroscopy. In this respect polycrystalline material of $\mathbf{5}$ was placed in a Mössbauer tablet and covered with mineral oil inside a glovebox. The sample was immediately flashfrozen and a spectrum was recorded at $80 \mathrm{~K}$ (Figure 6.8).

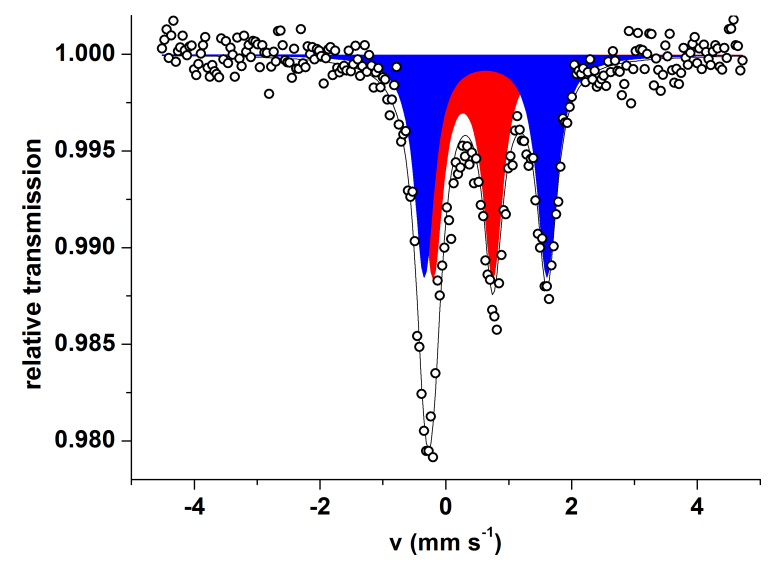

Figure 6.8: Mössbauer spectrum of a polycrystalline sample of $\mathbf{5}$ at $80 \mathrm{~K}$. Isomer shifts $\delta$ and quadrupole splittings $\left|\Delta E_{\mathrm{Q}}\right|$ in $\left[\mathrm{mm} \mathrm{s}^{-1}\right]$. Red subspectrum: $\delta=0.27,\left|\Delta E_{\mathrm{Q}}\right|=0.95$, blue subspectrum: $\delta=0.63,\left|\Delta E_{\mathrm{Q}}\right|=1.95$.

The data were fit with two subspectra in a 50:50 integral ratio. The blue subspectrum featured an isomer shifts and quadrupole splitting indicative for a $\{\mathrm{Fe}(\mathrm{NO})\}^{7}$ site with a spin state of $S=3 / 2$. The red doublet on the other hand exhibited a significantly lower isomer shift and a smaller quadrupole splitting. In comparison with bond lengths from the struc- 
ture determination and reported isomer shifts in literature 176/177] a $S=1 / 2$ spin state can be assumed. Additionally, the isomer shifts of $\mathbf{5}$ were calculated with structural information from crystallographic data. Using a B3LYP (CP(PPP)) basis set 168 the following parameters can be derived using the non optimized coordinates of the crystal structure of 5: $\delta=0.27 \mathrm{~mm} \mathrm{~s}^{-1},\left|\Delta E_{\mathrm{Q}}\right|=0.95 \mathrm{~mm} \mathrm{~s}^{-1}$ and $\delta=0.46 \mathrm{~mm} \mathrm{~s}^{-1},\left|\Delta E_{\mathrm{Q}}\right|=1.95 \mathrm{~mm} \mathrm{~s}^{-1}$.

Thus Mössbauer data affirm that the spin states for $\mathrm{Fe} 1$ and $\mathrm{Fe} 2$ in $\mathbf{5}$ differ. Calculation and experiment point to a $S=3 / 2$ and a $S=1 / 2$ system.

\section{Magnetic Susceptibility Measurement}

To finally substantiate the spin state and magnetic properties of $\mathbf{5}$, the variable temperature magnetic susceptibility data were determined via SQUID magnetometry. A polycrystalline sample of $\mathbf{5}$ was placed in a gel capsule and covered with fluorinated oil in a glovebox. The capsule was sealed with a lid and placed inside a PTFE straw for the measurement. The magnetization of the sample in a range of $2-220 \mathrm{~K}$ was recorded. All data were corrected for paramagnetic contributions and TIP. The data were fit with the program JulX 174] using a HEISENBERG DIRAC VAN VLECK hamiltonian including isotropic exchange interactions, zerofield- and Zeemann splitting. As fitting parameters the two spin states of $S=3 / 2$ and $S=1 / 2$ were applied. The closest agreement between fit and experiment was obtained assuming an antiferromagnetic coupling of the two iron sites $\left(J=-73.7 \mathrm{~cm}^{-1}\right)$. While values for the iron site with $S=3 / 2$ were freely refined with a zerofield splitting parameter of $D=-42.9 \mathrm{~cm}^{-1}$ and free $g$ values, for the other iron site fixed parameters with $g$ values of 2 and without zerofield splitting parameter were chosen. The best fit is shown in Figure 6.9 left. Additionally VTVH measurements at field strengths of $1 \mathrm{~T}, 3 \mathrm{~T}$ and $5 \mathrm{~T}$ were recorded (Figure 6.9 right). All data were simulated with the same parameters.
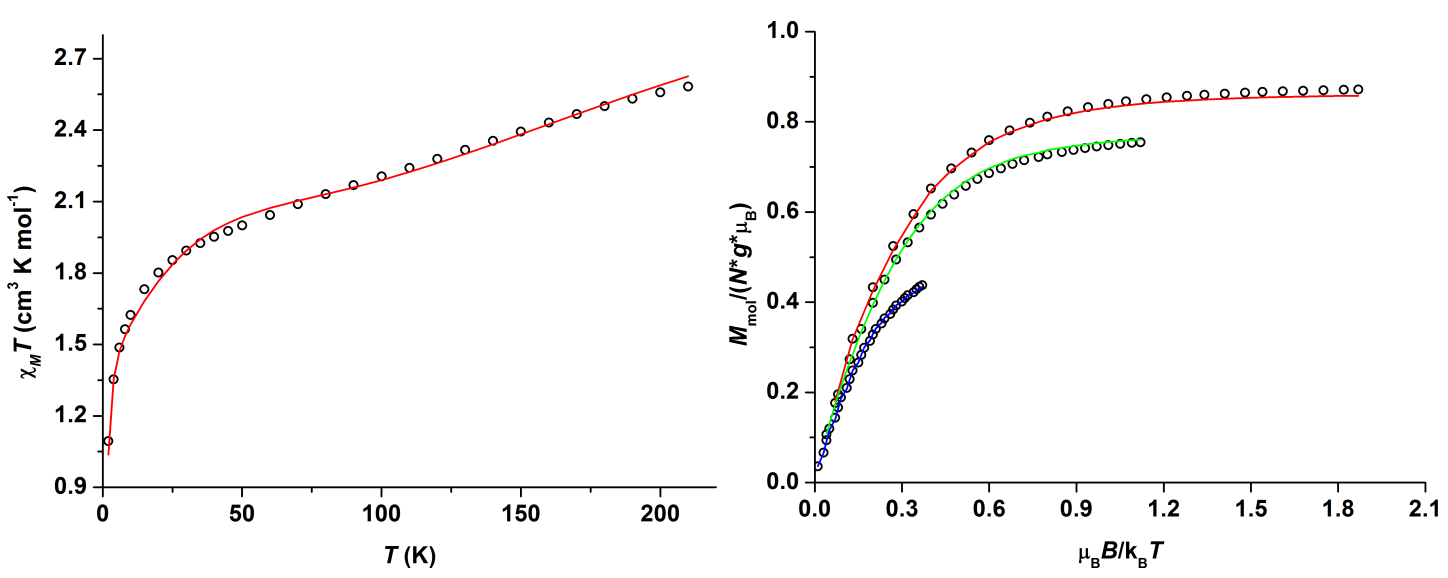

Figure 6.9: Magnetic susceptibility measurement of 5. Left: $\chi_{\mathrm{M}} T$ vs. $T$ measurement indicating an antiferromagnetic coupling of an $S=3 / 2$ and an $S=1 / 2$ state. Right: Variable Temperature Variable Field-measurement (VTVH) at field strengths of $1 \mathrm{~T}$ (blue), $3 \mathrm{~T}$ (green) and $5 \mathrm{~T}$ (red). The solid lines represent the best simultaneous fits for both data sets. 


\subsubsection{Summary}

In this section the synthesis and thorough characterization of two new diiron dinitrosyl $\left[\{\mathrm{Fe}(\mathrm{NO})\}^{7}\right]_{2}$-complexes $\mathbf{4}$ and $\mathbf{5}$ by means of x-ray crystallography, IR- and Mössbauer spectroscopy as well as magnetic measurements has been presented. All relevant data are compared with the two model systems in literature in Table 6.1 .

Table 6.1: Comparison of structural and spectroscopic parameters of $\mathbf{4}$ and $\mathbf{5}$ with literature known model systems.

\begin{tabular}{l|llll}
\hline & $\mathbf{4}$ & $\mathbf{5}$ & XI (Lippard) $[1]$ & XII (Lehnert) 103 \\
\hline $\mathrm{Fe} \cdots \mathrm{Fe}[\AA]$ & 4.37 & 4.45 & 3.44 & 3.47 \\
$\mathrm{~N}-\mathrm{O}[\AA]$ & $1.13,1.16$ & 1.17 & 1.15 & $1.16,1.17$ \\
$\mathrm{Fe}-\mathrm{N}-\mathrm{O}\left[^{\circ}\right]$ & $125-165$ & 147,155 & 167 & 156,145 \\
$\mathrm{~N} \cdots \mathrm{N}(\mathrm{NO})[\AA]$ & 3.04 & 2.92 & 2.82 & 2.80 \\
$\delta\left[\mathrm{mm} \mathrm{s}^{-1}\right]$ & $0.62,0.61$ & $0.27,0.63$ & 0.67 & - \\
$\left|\Delta \mathrm{E}_{\mathrm{Q}}\right|\left[\mathrm{mm} \mathrm{s}^{-1}\right]$ & $1.65,1.85$ & $0.95,1.95$ & 1.44 & - \\
$\tilde{\nu}\left[\mathrm{cm}^{-1}\right]$ & 1760,1733 & $1778+\mathrm{sh}$ & 1785 & 1760 \\
\hline
\end{tabular}

Interestingly, in 5 the diiron core was found to exhibit two different spin states $(S=1 / 2$ and $S=3 / 2$ ). This observation is unprecedented as all model systems to date as well as the active site in Flavodiiron proteins feature two iron sites in the high spin $(S=3 / 2)$ state. Also rarely observed has been a split band in the region of the NO-stretch in infrared spectra. This split can be according to DFT calculations attributed to a mechanical coupling of the two Fe-NO centers ${ }^{[83}$ resulting in an "in-phase" and "anti-phase" stretching motion. For pyrazolate based dinuclear iron dinitrosyl complexes this behavior has been observed before but has not been rationalized by DFT calculations. 128] Notably, structural parameters in $\mathbf{4}$ and $\mathbf{5}$ differ from previously reported model systems. The Fe $\cdots$ Fe distance is significantly larger and also $\mathrm{Fe}-\mathrm{N}-\mathrm{O}$ angles differ especially in 4. As the $\mathrm{Fe}$-.. Fe distance in $\mathbf{5}$ is larger than in $\mathbf{4}$ also a larger $\mathrm{N} \cdots \mathrm{N}$ ( $\mathrm{NO}$ units) separation might be anticipated. Intriguingly, this is not the case. The NO-moieties in $\mathbf{5}$ lie more "in plane" and thus in closer proximity. Since $\mathbf{4}$ and $\mathbf{5}$ bind nitric oxide in a syn fashion, in this respect, the two Fe-NO moieties in these complexes are ideally preorganized for the reduction of NO to $\mathrm{N}_{2} \mathrm{O}$. Hence, two new model systems that mimic the active site in Flavodiiron proteins were successfully established. 


\subsection{Electrochemical Properties and Reduction of Diiron Dinitrosyl Complexes}

The two new systems $\mathbf{4}$ and $\mathbf{5}$ were intentionally designed to mimic the reactivity of the active site of Flavodiiron proteins. FDPs are known to reduce nitric oxide to the less toxic $\mathrm{N}_{2} \mathrm{O}$. 96. The fully loaded FDP provides four electrons for the reduction of four NO molecules to two $\mathrm{N}_{2} \mathrm{O}$ molecules. Two electrons are made available by the diiron core and two by the FMN domain. 91 The reduction of two nitric oxide molecules with two protons thus requires two electrons to yield $\mathrm{N}_{2} \mathrm{O}$ and water. Hence, the diiron core alone, should be capable of mediating the reduction process of two NO molecules to $\mathrm{N}_{2} \mathrm{O}$ in the presence of protons.

To probe whether the newly synthesized systems are capable to mimic this reactivity and generate $\mathrm{N}_{2} \mathrm{O}$ upon reduction, cyclic voltammetry and IR-spectroelectrochemistry measurements were performed. The results will be discussed in the following sections.

\subsubsection{Cyclic Voltammetry and IR-Spectroelectrochemistry of 4}

Cyclic voltammetry is a helpful tool to gain a first insight into a compound's redox potentials. The cyclic voltammetric scans of the previously described diiron dinitrosyl complexes were carried out under the following conditions: For all measurements anhydrous and oxygen-free solvents were used. The measuring cell was sealed for the duration of a measurement ensuring a minimal exposure to dioxygen. The following electrodes were used: glassy carbon as working electrode, platinum as counterelectrode, and a silver wire as reference electrode. Tetrabutylammonium hexafluorophosphate was used as a conducting salt.

The cyclic voltammograms of 4 in a solvent mixture of DCM/MeOH 5:1 are depicted in Figure 6.10 .
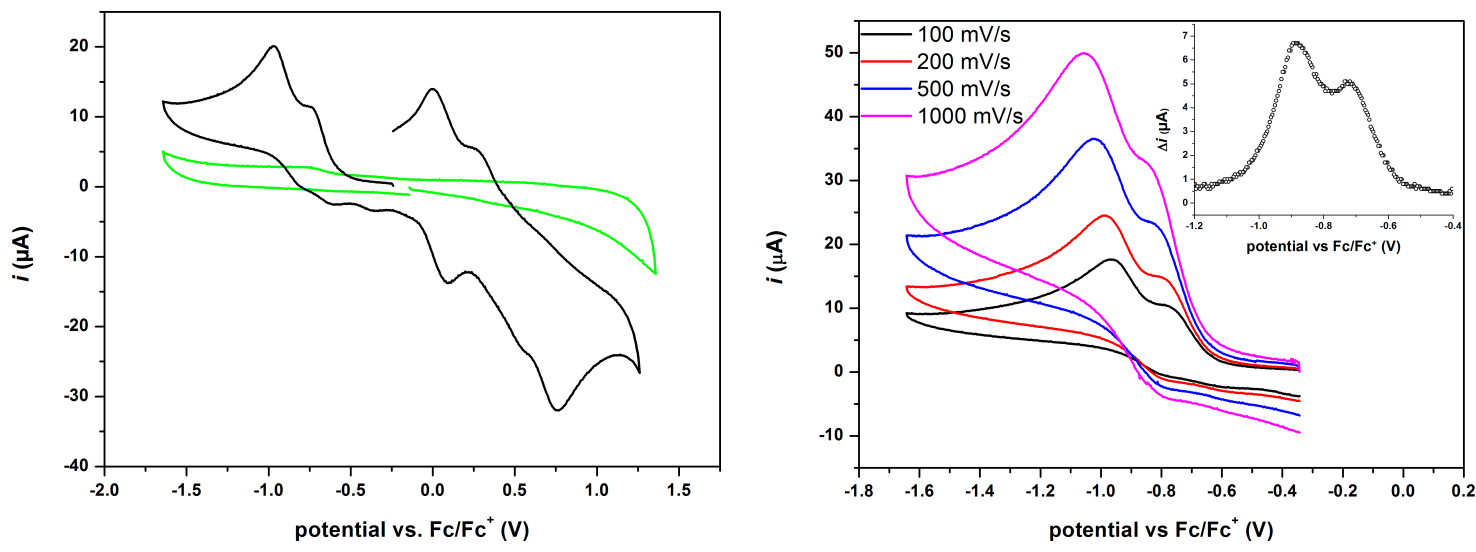

Figure 6.10: Cyclic voltammograms of 4 in $\mathrm{DCM} / \mathrm{MeOH} 5: 1\left(0.1 \mathrm{M} \mathrm{NBu} 4 \mathrm{PF}_{6}\right)$. The potential is referenced with the ferrocene/ferrocenium couple under the same conditions. Left: Full cyclic voltammogram (black line) compared to solvent background (green line). Right: Cathodic reduction wave at different scan rates. The inset shows the corresponding square wave voltammogram. 
The full cyclic voltammogram of $\mathbf{4}$ can be divided into two sets of signals. One part is described by the cathodic reduction below $-0.2 \mathrm{~V}$ vs. $\mathrm{Fc} / \mathrm{Fc}^{+}$and the other part by an anodic oxidation. The anodic oxidation event above $-0.2 \mathrm{~V}$ was observed on the reverse scan exhibits signals which can most likely be assigned to a product after the irreversible reduction of $\mathbf{4}$ and/or diiron species without coordinated nitric oxide. Strikingly, similar oxidation events were also observed when scanning the anodic oxidation without prior reduction. One explanation might be that in solution and upon applying a potential, a fraction of the coordinated NO ligands dissociates, which would impose a similar effect. A magnification of the cathodic reduction event reveals two reduction processes at $-0.95 \mathrm{~V}$ and $\sim-0.77 \mathrm{~V}$ vs. $\mathrm{Fc} / \mathrm{Fc}^{+}(100 \mathrm{mV} / \mathrm{s})$. The two irreversible signals could describe a sequential reduction of the two nitric oxide moieties of 4 . Additional square wave voltammetry confirmed the occurrence of a sequential two step reduction process. Generally, for a sequential reduction with two electrons two square wave signals of the same height and integral area would be expected. However, since the reduction process is irreversible square wave voltammetry does not provide reliable information about these events. It can be interpreted as a first indication that the irreversible reduction of $\mathbf{4}$ might require two sequential reduction steps.

A solvent mixture of DCM/MeOH 5:1 was chosen for the recorded cyclic voltammograms for the following reasons: Firstly in pure methanol, the complexes $\mathbf{4}$ and $\mathbf{5}$ lost their coordinated nitric oxide ligands over time and secondly, the solubility of the conducting salt $\mathrm{NBu}_{4} \mathrm{PF}_{6}$ and the complexes was found to be very poor in pure methanol. With regard to additionally planned solution IR electrochemical measurements pure methanol did moreover not provide an adequate solvent window as it featured strong absorbance bands at wavenumbers in which stretching frequencies of coordinated nitrosyls were expected. Methanol was nevertheless added to DCM in small quantities to provide a proton source which might facilitate NO reduction.

To obtain a more detailed insight into the reduction process, the reduction of $\mathbf{4}$ was followed by IR-spectroscopy. In a solution IR cell a potential of $-1.2 \mathrm{~V}$ vs. silver wire was applied to a solution of 4 in DCM/MeOH 5:1. Figure 6.11 illustrates the obtained spectra.

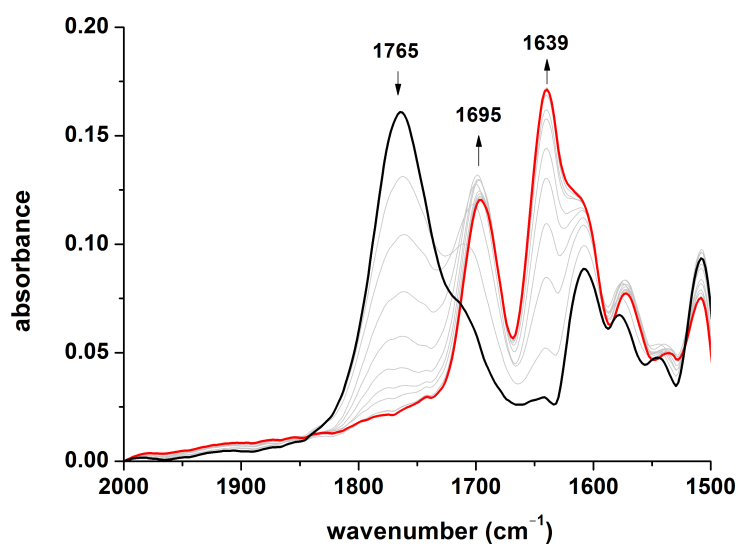

Figure 6.11: Electrochemical reduction followed by IR-spectroscopy in a DCM/MeOH 5:1-mixture with an applied potential of $-1.5 \mathrm{~V}$.

A broad signal at $1765 \mathrm{~cm}^{-1}$ indicates the nitrosyl stretching frequency of the Fe-NO moieties (Figure 6.11 left, black line). Upon applying a potential of $-1.5 \mathrm{~V}$, which should 
be sufficient to implement NO reduction, this band decreases. The red line indicates a final reduced species. Weak new bands appear at roughly 1639 and $1695 \mathrm{~cm}^{-1}$, which could be a first indication for the formation of a diiron tetranitrosyl complex.

\subsubsection{Chemical Reduction of 4}

In addition to the electrochemistry experiments 4 can also be reduced chemically. A suitable reducing agent in this respect is cobaltocene $\left(\mathrm{Cp}_{2} \mathrm{Co} / \mathrm{Cp}_{2} \mathrm{Co}^{+}\right.$couple $-1.33 \mathrm{~V}$ vs. $\mathrm{Fc} / \mathrm{Fc}^{+}$in DCM). ${ }^{178}$ The reducing strength of cobaltocene should be sufficient to reduce 4 entirely. Following the chemical reduction with an excess of $\mathrm{Cp}_{2} \mathrm{Co}$ by IR spectroscopy in solution the initial NO stretching band disappears and two new signals $(\tilde{\nu}=1631$ and $1684 \mathrm{~cm}^{-1}$ ) emerge. Figure 6.12 left depicts the corresponding spectra.
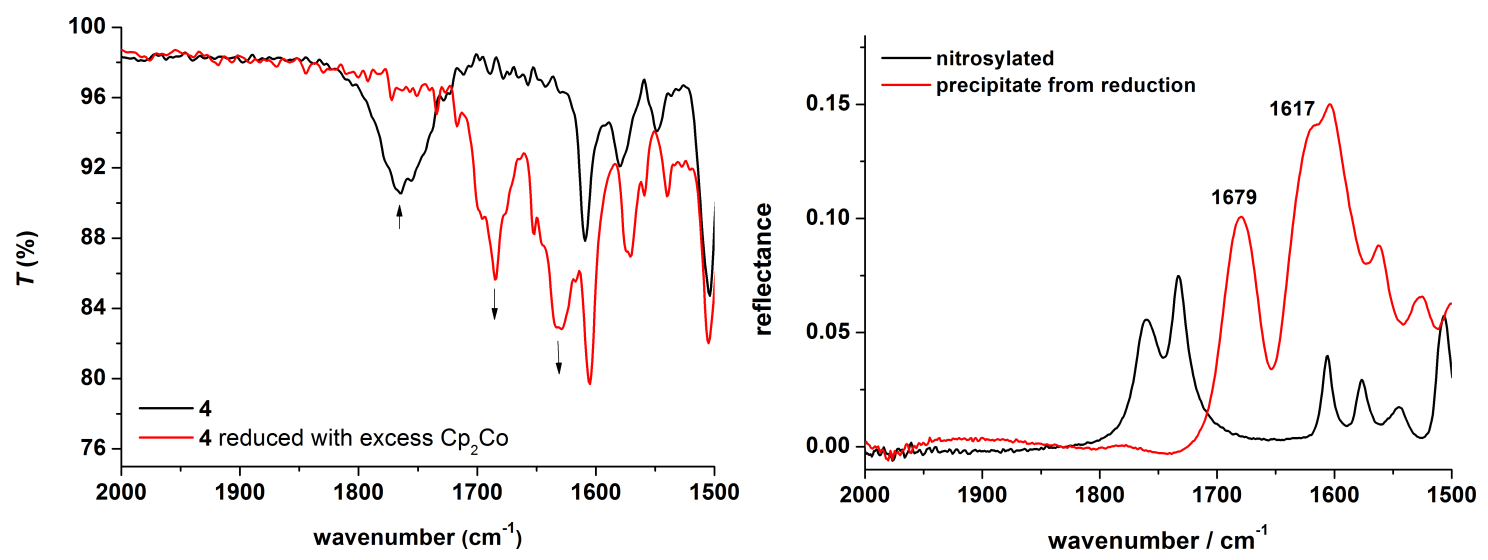

Figure 6.12: Left: IR-spectrum of 4 in DCM/MeOH. The black line indicates the initial spectrum while the red line represents the fully reduced species after addition of $\mathrm{Cp}_{2} \mathrm{Co}$. Right: ATRspectrum of crystalline material of $\mathbf{4}$ (Black line) and the isolated precipitate after reduction (red line).

The resolution of the depicted IR spectrum (Figure 6.12 left), however, is very poor and despite baseline correction the entire spectrum seems to shift upon reduction. To investigate the nature of the two new signals at $\sim 1631$ and $1683 \mathrm{~cm}^{-1}$ in the infrared spectrum, a larger amount of $\mathbf{4}$ was reduced in methanol solution. In the course of this reaction the initially brown solution turned red and a precipitate formed. The suspension was filtered and the collected solid analyzed by ATR-spectroscopy. The resulting spectrum is shown in Figure 6.12 (right). The precipitate features two main IR bands at approximately $\tilde{\nu}=1679$ and $1617 \mathrm{~cm}^{-1}$. The band at $\tilde{\nu}=1679 \mathrm{~cm}^{-1}$ is very similar to the fully reduced product (Figure 6.12 left, red line). Prominent bands in this range might be attributed to the formation of a tetranitrosyl diiron species (two nitrosyl units per iron center (DNIC)). $179 \mid 180$ Typically DNICs feature two prominent IR-bands, which are according to literature separated by $15-38 \mathrm{~cm}^{-1}$ in dinuclear complexes. 180 . A formation of a tetranitrosyl diiron complex, a $\left[\{\mathrm{Fe}(\mathrm{NO})\}^{9}\right]_{2}$ species, was observed by KINDERMANN for pyrazolate bridged diiron complexes before. 128 .

As described above, when reducing 4 in methanol with $\mathrm{Cp}_{2} \mathrm{Co}$, aside from the formation and analysis of the precipitate, also the entire reaction solution changed color from brown to red. After filtration the red filtrate in methanol was left for crystallization by diethyl 
ether diffusion. Single crystals suitable for x-ray diffraction were grown within a couple of days. The obtained reduction product, which is soluble in methanol is a methanolate bridged Fe(II)Fe(II)- complex $\mathbf{4}^{\text {Red }} .4^{\text {Red }}$ crystallizes in the triclinic space group $P \overline{1}$ with one complex cation and two triflate counterions in the unit cell. The $\mathrm{Fe} \cdots \mathrm{Fe}$ distance lies is $3.75 \AA$. Fe-N distances vary between $2.07 \AA$ for the pyrazolate and $2.56 \AA$ for the amine nitrogen donors of the side arms. The $\mathrm{Fe}-\mathrm{O}$ bond lengths are not equal. The methanolate bridge is located slightly closer to Fe1 than to Fe2. Figure 6.13 depicts the complex cation, which is twofold positively charged.

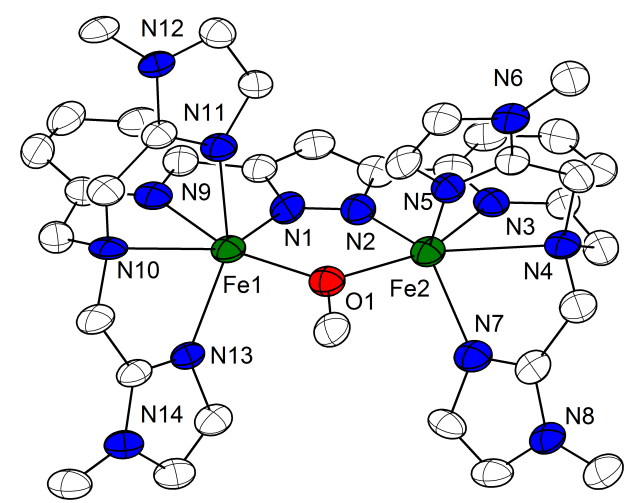

Figure 6.13: Cation of the molecular structure of $\left[\mathrm{L}^{\mathrm{Im}} \mathrm{Fe}_{2}(\mu-\mathrm{OMe})\right](\mathrm{OTf})_{2} \mathbf{4}^{\mathbf{R e d}}$. Thermal ellipsoids are set to $50 \%$. Hydrogen atoms as well as the triflate anions were omitted for clarity. Selected bond lengths $[\AA]$ and angles [ $\left.{ }^{\circ}\right]$ Fe1-O1 2.031(5), Fe1-N1 2.076(6), Fe1-N9 2.268(6), Fe1N10 2.463(5), Fe1-N11 2.174(5), Fe1-N13 2.132(5), Fe2-O1 2.176(4), Fe2-N2 2.064(6), Fe2-N3 2.340(6), Fe2-N4 2.562(9), Fe2-N5 2.119(5), Fe2-N7 2.130(6), Fe1-O1-Fe2 126.3(2), Fe1-N1-N2$\mathrm{Fe} 2-2.271(9)$.

From a comparison of $\mathrm{Fe}-\mathrm{N}$ bond lengths a high spin configuration can be expected for both iron atoms. 140 If 4 is chemically reduced with $\mathrm{Cp}_{2}$ Co in pure DCM solution, due to the absence of methanol, the chloride bridged analogue $\mathbf{4} \mathbf{b}^{\mathbf{R e d}}$ is obtained (Appendix 11.4.1, which will not be discussed in detail in this chapter.

To unambigously establish the spin state in $\mathbf{4}^{\text {Red }}$ zerofield Mössbauer spectroscopy was performed of solid crystalline material and in solution. The respective spectra are depicted in Figure 6.14. In solid state two very similar subspectra for two $\mathrm{Fe}$ (II) high spin sites can be observed. The slight difference in isomer shift and quadrupole splitting can be explained by minor structural differences for the two iron centers in the crystal lattice. The occurence of only one signal in frozen solution can be attributed to an enhanced flexibility of the coordination environment in solution. In that case the two iron centers can be regarded as coordinatively equal.

IR analysis was performed of $\mathbf{4}^{\text {Red }}$ to rule out that the isolated precipitate and the crystallographically characterized complex from the filtrate were the same compound. The spectrum is shown in Figure 6.15. No nitrosyl stretching bands are apparent in the spectrum of $\mathbf{4}^{\mathbf{R e d}}$. Hence the precipitate and $\mathbf{4}^{\mathbf{R e d}}$ must differ in their molecular structure. 

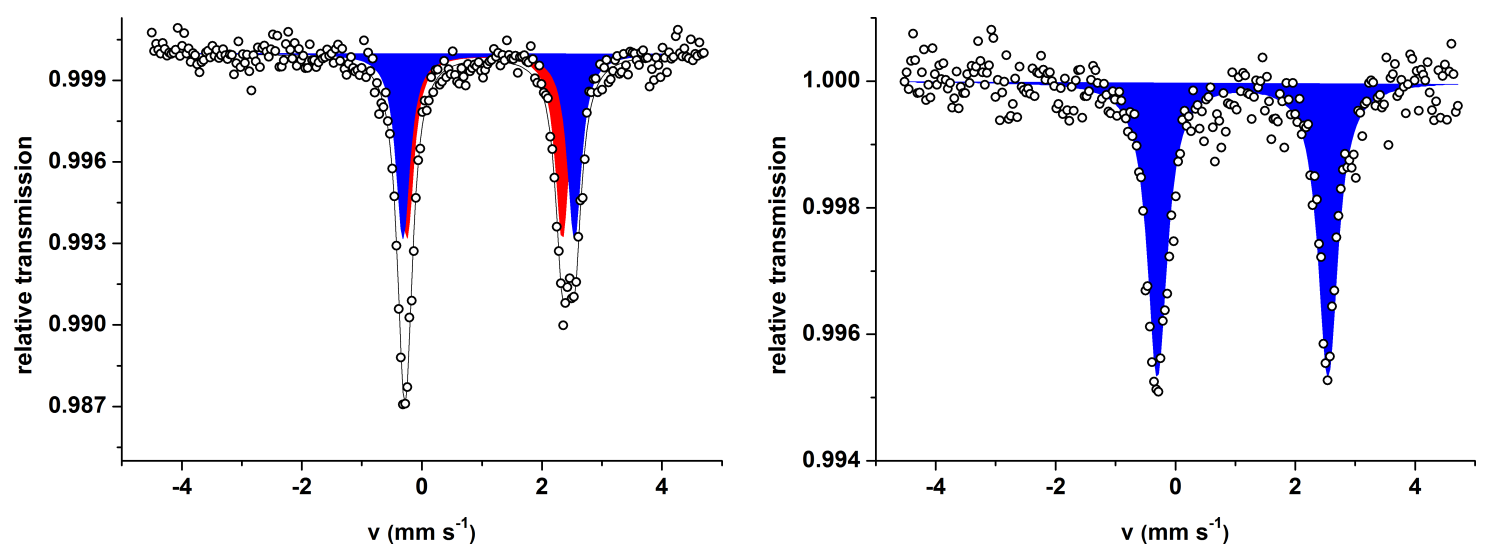

Figure 6.14: Zerofield Mössbauer spectra of $\mathbf{4}^{\text {Red }}$ at $80 \mathrm{~K}$. Isomer shifts $\delta$ and quadrupole splittings $\left|\Delta E_{\mathrm{Q}}\right|$ in $\left[\mathrm{mm} \mathrm{s}^{-1}\right]$. Left: Solid state spectrum. Red subspectrum: $\delta=1.04,\left|\Delta E_{\mathrm{Q}}\right|=2.59$, blue subspectrum: $\delta=1.11,\left|\Delta E_{\mathrm{Q}}\right|=2.86$. Right: $\mathbf{4}^{\text {Red }}$ in $\mathrm{MeOH}$ solution. $\delta=1.12,\left|\Delta E_{\mathrm{Q}}\right|=2.85$

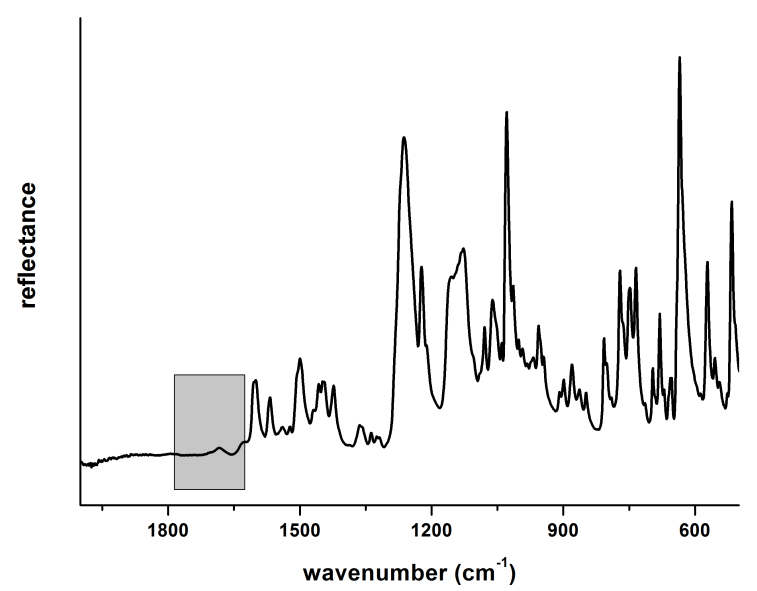

Figure 6.15: ATR-spectrum of crystalline material of $4^{\text {Red }}$. The grey area highlightes the region in which nitrosyl stretches would be expected.

Combining all collected data for the electrochemical and chemical reduction of 4 a putative reaction pathway can be postulated. IR data point towards the formation of a second nitrosylated species upon reduction, which might be attributed to the formation of a tetranitrosyl diiron complex $\left[\left\{\mathrm{Fe}(\mathrm{NO})_{2}\right\}^{9}\right]_{2}$. However, as the precipitate was never isolated in sufficient yields as crystalline material for x-ray analysis, this formation is so far not fully substantiated. From the chemical reduction it is furthermore known that $\mathbf{4}^{\text {Red }}$ is formed aside from the nitrosylated species. Taking all these findings into account a possible reaction scenario as illustrated in Scheme 6.2 can be formulated.

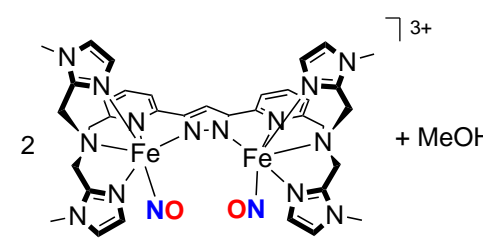

4

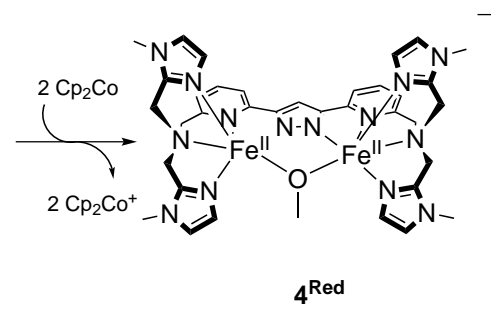

$4^{\text {Red }}$

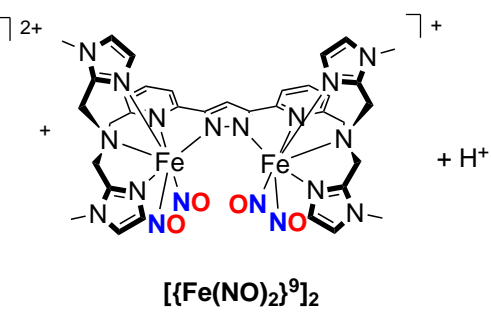

$\left[\left\{\mathrm{Fe}(\mathrm{NO})_{2}\right\}^{9}\right]_{2}$

Scheme 6.2: Possible reduction pathway for 4 . 


\subsubsection{Cyclic Voltammetry and IR-Spectroelectrochemistry of 5}

The cyclic voltammograms of $\mathbf{5}$ were recorded in analogy to $\mathbf{4}$ in a solvent mixture of DCM/MeOH 5:1 (0.1 M NBu $\left.4 \mathrm{PF}_{6}\right)$ with the intention to gain comparable data for all analytical techniques including IR analysis, which is limited to a great extent by the absorbance of the solvent. As working and counter electrode a glassy carbon and a platinum electrode were chosen, respectively. A silver wire was used as reference electrode. All spectra were recorded vs. the $\mathrm{Fc} / \mathrm{Fc}^{+}$couple.
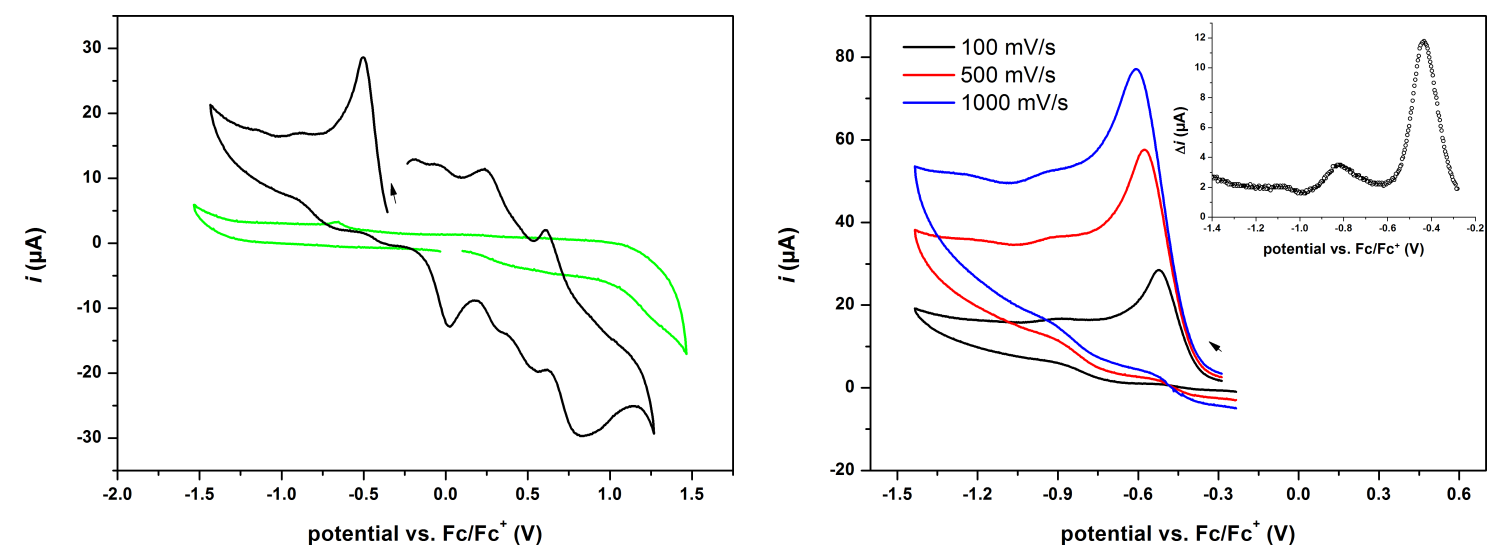

Figure 6.16: Cyclic voltammograms of 5 in $\mathrm{DCM} / \mathrm{MeOH}$ 5:1 (0.1 M NBu $\left.4 \mathrm{PF}_{6}\right)$. Left: Full voltammogram (black line) in comparison to background (green line). Right: Cathodic reduction wave at different scan rates. The inset depicts the corresponding square wave diagram.

The full voltammogram of $\mathbf{5}$ exhibits a prominent irreversible reduction wave at $-0.52 \mathrm{~V}$ vs. the $\mathrm{Fc} / \mathrm{Fc}^{+}$couple $(100 \mathrm{mV} / \mathrm{s})$ and several oxidation processes in the backscan of the cyclic voltammogram, which can be most likely attributed to a product after the irreversible reduction event. The reduction of $\mathbf{5}$ is evidently shifted to more positive potentials compared to 4 . When examining the reduction process closer though, one or two minor waves can be observed at very low potentials succeeding the first very prominent wave. Hence extracting information on the number of electrons being transferred during reduction and the reduction process itself is not possible from cyclic voltammetry.

The reduction of $\mathbf{5}$ was moreover followed by IR-spectroelectrochemistry. In a conducting salt solution of the same composition as for cyclic voltammetry, a potential of $-1.5 \mathrm{~V}$ was applied. The magnitude of the applied potential should be sufficient to go beyond the last reduction process and ensure full reduction of $\mathbf{5}$. The recorded spectra are illustrated in Figure 6.17

The initial IR spectrum of $\mathbf{5}$ in solution (black line) reveals a broad band at $1776 \mathrm{~cm}^{-1}$, which can be assigned to the NO-stretches of $\mathbf{5}$ in solution. Upon reduction this band gradually decreases and disappears. Apart from a strong baseline shift of the spectrum, the fully reduced species does not display any new bands in the range of 1500 to $2000 \mathrm{~cm}^{-1}$. Hence, the coordinated NO moieties are "removed" upon reduction and do not seem to rebind to the diiron complex, as no additional nitrosyl stretches were observed.

Larger amounts of $\mathbf{5}$ were reduced chemically to ascertain more information about the reduction process and the whereabouts of $\mathrm{NO}$, which is discussed in the following: 


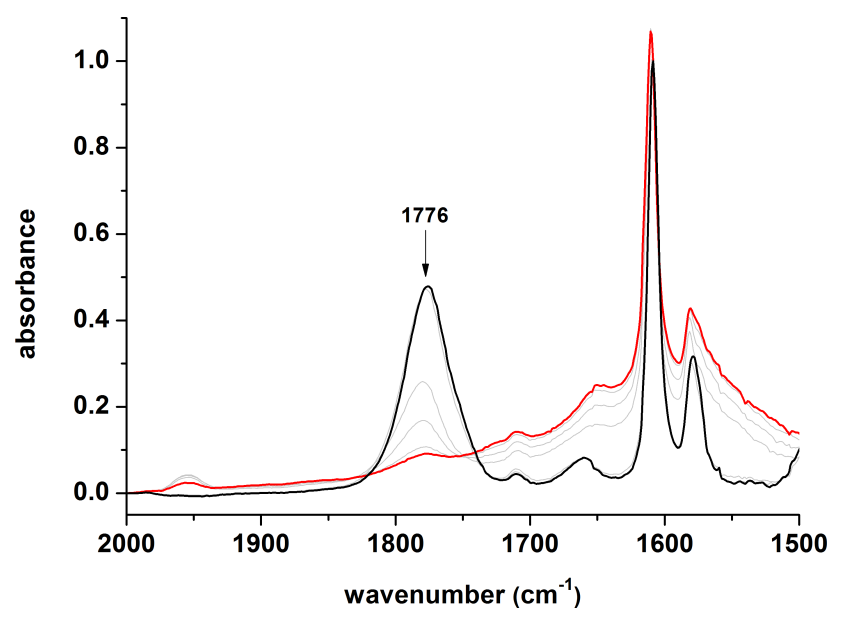

Figure 6.17: Electrochemical reduction of $\mathbf{5}$ at an applied potential of $-1.5 \mathrm{~V}$ followed by IR spectroscopy in a DCM/MeOH 5:1 mixture. Initial spectrum of $\mathbf{5}$ is highlighted by a black line. Fully reduced $\mathbf{5}$ is highlighted in red.

\subsubsection{Chemical Reduction of 5}

In a first experiment a larger amount of $\mathbf{5}$ was reduced with excess $\mathrm{Cp}_{2}$ Co in methanol. The reducing power of $\mathrm{Cp}_{2} \mathrm{Co}\left(\mathrm{Cp}_{2} \mathrm{Co} / \mathrm{Cp}_{2} \mathrm{Co}^{+}\right.$couple $-1.33 \mathrm{~V}$ vs. $\mathrm{Fc} / \mathrm{Fc}^{+}$in $\left.\mathrm{DCM}\right)$ should be adequate to ensure the full reduction of 5 . 178 In the course of this reaction the initally dark brown solution of the diiron dinitrosyl complex turned red within minutes. Other than for the reduction of $\mathbf{4}$ no precipitate was observed. Diethyl ether diffusion into the reaction solution afforded single crystals suitable for x-ray diffraction. A methanolmethanolate bridged diiron(II) complex $5^{\text {Red }}$ was obtained.

$5^{\text {Red }}$ crystallizes in the monoclinic space group $P 2_{1}$ with one complex cation and two triflate anions in the asymmetric unit. The two iron sites are separated by $4.50 \AA$. Fe-N distances vary between 2.00-2.30 $\AA$. The cationic portion of $\mathbf{5}^{\text {Red }}$ is depicted in Figure 6.18

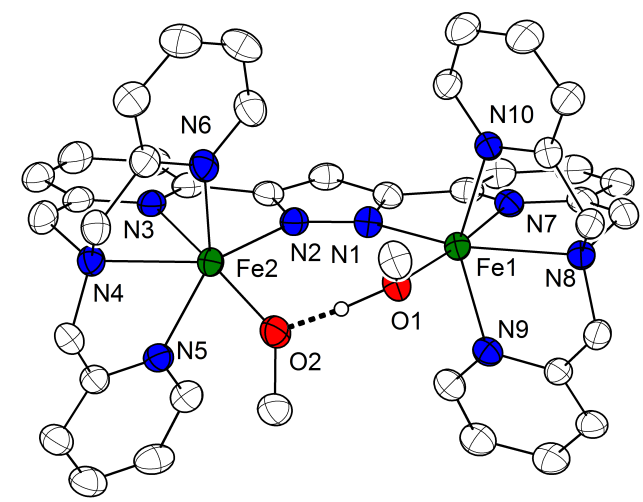

Figure 6.18: Cation of the molecular structure of $\left[\mathrm{L}^{\mathrm{Py}} \mathrm{Fe}_{2}(\mu-\mathrm{MeOH}-\mathrm{OMe})\right](\mathrm{OTf})_{2} \mathbf{5}^{\text {Red }}$. Thermal ellipsoids are set to $50 \%$. Irrelevant hydrogen atoms as well as the triflate anions were omitted for clarity. Selected bond lengths $[\AA]$ and angles $\left[^{\circ}\right]$ Fe1-O2 1.993(3), Fe1-N1 2.166(3), Fe1N7 2.170(3), Fe01-N8 2.292(3), Fe1-N9 2.211(3), Fe1-N10 2.211(3), Fe2-O1 2.003(3), Fe2-N2 2.165(3), Fe2-N3 2.145(3), Fe2-N4 2.298(3), Fe2-N5 2.205(3), Fe2-N6 2.172(3), Fe1-N1-N2-Fe2 $-13.909(5)$. 
Fe-N bond lengths point to a high spin state for both iron sites in $\mathbf{5}^{\text {Red }}$. To verify this assumption a zerofield Mössbauer spectrum was recorded at $80 \mathrm{~K}$. The respective spectrum is depicted in Figure 6.19 left.
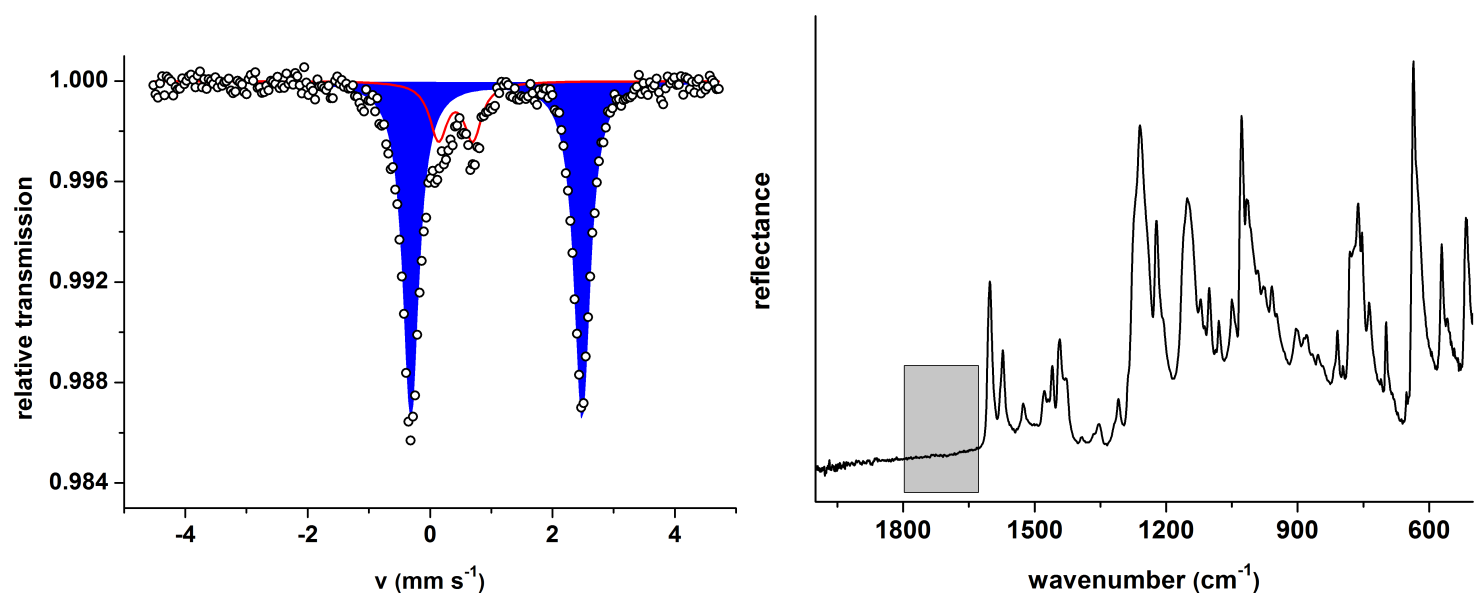

Figure 6.19: Left: Zerofield Mössbauer spectrum of a polycrystalline sample of $\mathbf{5}^{\text {Red }}$ at $80 \mathrm{~K}$. Isomer shifts $\delta$ and quadrupole splittings $\left|\Delta E_{\mathrm{Q}}\right|$ in $\left[\mathrm{mm} \mathrm{s}^{-1}\right] . \quad \delta=1.09,\left|\Delta E_{\mathrm{Q}}\right|=2.80$. The red subspectrum indicates an impurity of $12 \%$ possibly of a Fe(III) species. Right: IR spectrum of $5^{\text {Red }}$. The grey box highlights the region in which nitrosyl stretches would appear. Hence, no nitrosylated portions can be found in $\mathbf{5}^{\text {Red }}$.

One main Mössbauer doublet can be obtained for $\mathbf{5}^{\text {Red }}$. This main species corresponds to a Fe(II) high spin signal with very similar isomer shift and quadrupole splitting as found for $4^{\text {Red }}$. An impurity of $12 \%$ (supposably an Fe(III) side product) was inseparable from the crystalline material even after many recrystallization attempts inside a glovebox. For the Mössbauer spectrum of $5^{\text {Red }}$ a representation with only one subspectrum was chosen, as bond lengths for $\mathrm{Fe} 1$ and $\mathrm{Fe} 2$ in the crystal structure did not significantly differ. Figure 6.19 right shows the IR spectrum of $\mathbf{5}^{\text {Red }}$. As expected, no nitrosyl bands are visible in the product.

Differently from 4 , no second reduction product was isolated from the chemical reduction of $\mathbf{5}$ with $\mathrm{Cp}_{2} \mathrm{Co}$ aside from $\mathbf{5}^{\text {Red }}$. Evidently, the $\mathrm{NO}$ coligands were removed in the course of the reduction. For the removal of NO several pathways are possible. One is the formation of a nitrosylated $\left[\left\{\mathrm{Fe}(\mathrm{NO})^{9}\right\}\right]_{2}$ species as postulated for the reduction of $\mathbf{4}$, which would be apparent from prominent stretching frequencies in the IR spectrum. As no new IR bands emerged upon reduction this pathway can be disregarded. A second option could involve the dissociation of $\mathrm{NO}$ as $\mathrm{NO}^{-}$. $\mathrm{NO}^{-}$would be detectable in IR spectra exhibiting a stretching frequency at $\tilde{\nu}=1470 \mathrm{~cm}^{-1} .[80$ In the present solution spectra (Figure 6.17) this frequency would be masked by absorbances of the solvent and bands of the ligand backbone.

More likely though is the formation of $\mathrm{N}_{2} \mathrm{O}$ which would evolve from the reaction mixture and thus would not be traceable in solid and only to a small extent in liquid samples. To probe this third reactivity pathway, attempts were made to detect $\mathrm{N}_{2} \mathrm{O}$. 


\subsection{5 $\mathrm{N}_{2} \mathrm{O}$ Detection}

To rule out the mere dissociation of NO in solution, the stability of $\mathbf{5}$ was tested in pure methanol and in the mixture DCM/MeOH 5:1 by means of UV/Vis spectroscopy. Spectra were recorded over a course of $12 \mathrm{~h}$ at $24.5^{\circ} \mathrm{C}$ in a Schlenk cuvette. The sample solution was stirred the entire time. In pure methanol the spectrum of $\mathbf{5}$ changed over this time course as the absorbance particularly in the range between $\lambda=420-520 \mathrm{~nm}$ decreased. In the $\mathrm{DCM} / \mathrm{MeOH}$ mixture UV/Vis bands basically remained the same. Results of the two UV/Vis experiments are depicted in Figure 6.20.
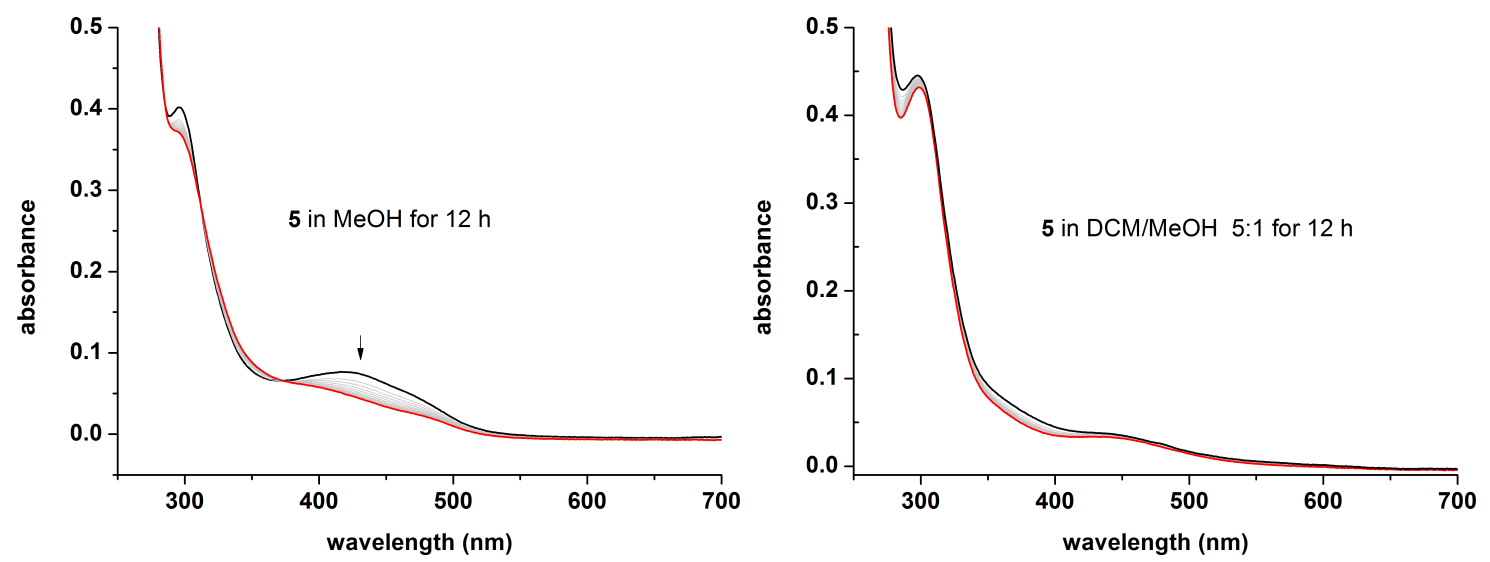

Figure 6.20: UV/Vis spectra of 5 over the course of 12 h. Left: in MeOH. Right: in DCM/MeOH 5:1. The initial spectrum is always colored in black, the final spectrum in red.

The solvent mixture seemed to be better suitable to ensure the stability of $\mathbf{5}$ in solution. For this reason, subsequent experiments for the detection of $\mathrm{N}_{2} \mathrm{O}$ were carried out in $\mathrm{DCM} / \mathrm{MeOH}$ 5:1. To gain an insight into how many equivalents of $\mathrm{Cp}_{2} \mathrm{Co}$ are needed to fully reduce 5 , a $\mathrm{UV} / \mathrm{Vis}$ titration with addition of $\mathrm{Cp}_{2} \mathrm{Co}$ in steps of 0.2 equivalents was carried out. Up to 1.0 eq. of $\mathrm{Cp}_{2} \mathrm{Co}$ the absorbance in the UV/Vis spectrum of $\mathbf{5}$ increased in the range of $\lambda=420-520 \mathrm{~nm}$ with isosbestic points at 339 and $375 \mathrm{~nm}$ (Figure 6.21). Upon further addition of $\mathrm{Cp}_{2} \mathrm{Co}$, the absorption maximum at $409 \mathrm{~nm}$ (red line) solely increased in intensity and shifted to $405 \mathrm{~nm}$. This behavior can be explained by the fact that the $\mathrm{Cp}_{2} \mathrm{Co}^{+}$cation features a prominent absorption maximum in this range for this solvent composition $(\lambda=405 \mathrm{~nm})$. This absorption band of $\mathrm{Cp}_{2} \mathrm{Co}^{+}$, however, masks all further spectral changes of $\mathbf{5}$ (For the UV/Vis spectra of $\mathrm{Cp}_{2} \mathrm{Co}$ and $\mathrm{Cp}_{2} \mathrm{Co}^{+}$ see Appendix 11.4.6.

To circumvent this effect, the absorbance of $\mathbf{5}$ at $470 \mathrm{~nm}$ was plotted vs. the added equivalents of $\mathrm{Cp}_{2} \mathrm{Co}$ (Figure 6.21 inset). Wavelengths above $470 \mathrm{~nm}$ lie outside the spectral range for $\mathrm{Cp}_{2} \mathrm{Co}^{+}$and thus only provide information about 5 . From this graph it is apparent that two equivalents of $\mathrm{Cp}_{2} \mathrm{Co}$ and consequently two electrons are required to fully reduce 5 .

After these preliminary experiments, the identification of $\mathrm{N}_{2} \mathrm{O}$ in the course of reduction was performed via headspace IR gas analysis. A setup (see also Appendix 11.4.7) and protocol for this measurement was implemented to obtain reliable data. First, the gas 


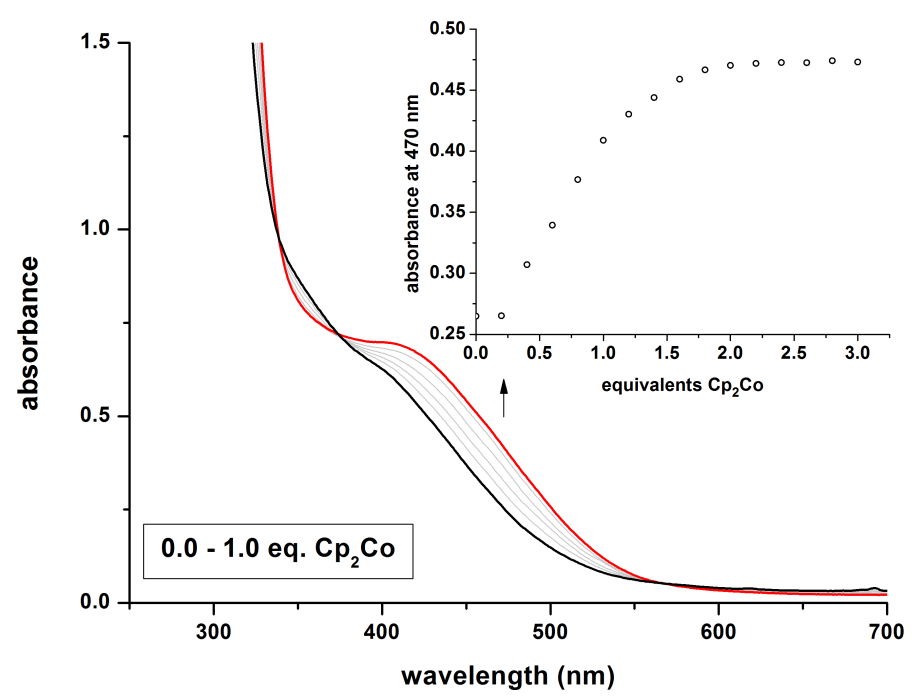

Figure 6.21: UV/Vis titration of $\mathbf{5}$ with $\mathrm{Cp}_{2} \mathrm{Co}$. The absorbance of the initial spectrum (black line) increases with exceeding equivalents of $\mathrm{Cp}_{2} \mathrm{Co}$ up to a 1:1 ratio (red line). The inset depicts the absorbance at $470 \mathrm{~nm}$ in dependence of added equivalents of the reduction agent.

IR cell with $\mathrm{KBr}$ windows was evacuated on a Schlenk line to $2 \cdot 10^{-2}$ mbar. To ensure consistency, the cell was always evacuated to the same pressure value. A two necked Schlenk tube was equipped with the evacuated gas IR cell and a septum in a glovebox filled with nitrogen and oxygen levels below $0.1 \mathrm{ppm}$. After the assembly of the Schlenk tube, cell and septum in the glovebox, the tube was filled with $6 \mathrm{~mL}$ of dry solvent $(\mathrm{DCM} / \mathrm{MeOH}$ 5:1). The valve to the IR cell was opened for exactly $90 \mathrm{~s}$ which is sufficient for equilibration between Schlenk tube and cell. After that the valve was closed and a blanc spectrum of the solvent headspace recorded. For the measurement of a sample the procedure was similar. The assembled setup was filled with $5 \mathrm{~mL}$ of a sample solution. $1 \mathrm{~mL}$ of solvent was used to dissolve an excess amount of $\mathrm{Cp}_{2} \mathrm{Co}$ and was added to the complex solution via a septum. The reaction mixture was allowed to stirr for several minutes. After that the valve of the IR cell was opened again for exactly $90 \mathrm{~s}$. Then the gasphase was analyzed by IR spectroscopy. Indeed, in this experiment $\mathrm{N}_{2} \mathrm{O}$ was detectable in the headspace of the reaction mixture. The $\mathrm{N}_{2} \mathrm{O}$ signal in the gas phase is marked by a split IR absorption band at 2236 and $2212 \mathrm{~cm}^{-1}$, respectively. The headspace IR spectra in this range for four different amounts of $\mathbf{5}$ are shown in Figure 6.22.

To check whether the obtained amount of $\mathrm{N}_{2} \mathrm{O}$ formed quantitatively in relation to the mole fraction of molecules of the deployed complex 5, a calibration curve had to be recorded. For this purpose gaseous $\mathrm{N}_{2} \mathrm{O}$ from a lecture bottle was used. Schlenk tube, IR cell and septum were assembled in a glovebox as previously described. The setup was filled with $6 \mathrm{~mL}$ of the solvent mixture. After that the entire, sealed setup was transferred out of the glovebox. A well defined volume of $\mathrm{N}_{2} \mathrm{O}$ was injected with a lockable Hamilton syringe into the headspace of the solution. After $3 \mathrm{~min}$ the valve to the IR cell was opened and allowed to equilibrate for exactly $90 \mathrm{~s}$. After that the IR gas cell was unmounted and the gasphase analyzed. This procedure was performed for six different volumes of $\mathrm{N}_{2} \mathrm{O}$. The calibration was repeated three times so that an average of three data sets for every volume was obtained. The mole fraction of injected $\mathrm{N}_{2} \mathrm{O}$ molecules is plotted in 


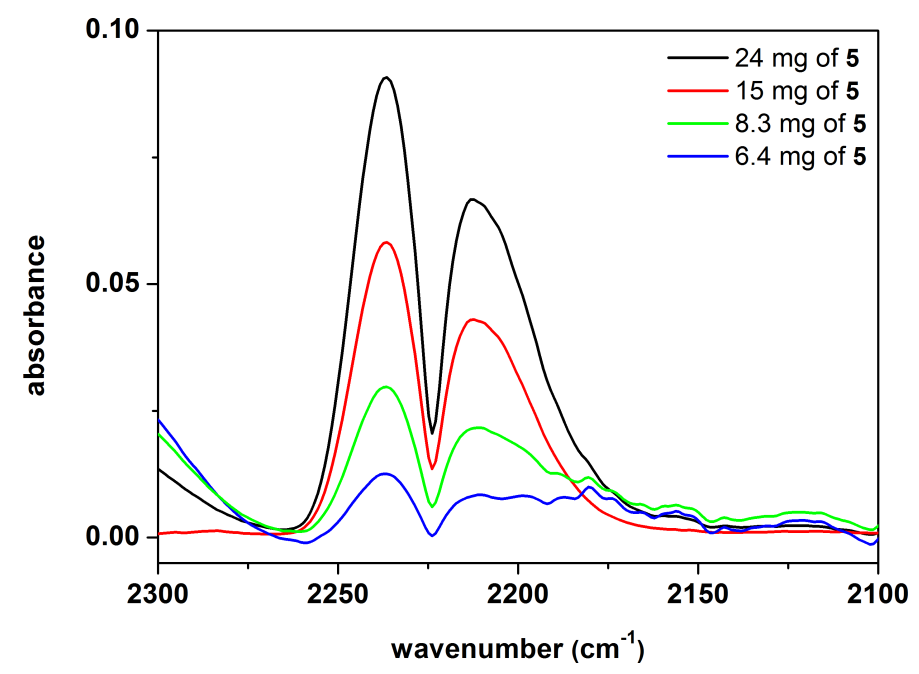

Figure 6.22: Excerpt of the headspace IR spectra of the reduction of four different samples of 5 . The characteristic $\mathrm{N}_{2} \mathrm{O}$ signals are at 2236 and $2212 \mathrm{~cm}^{-1}$, respectively.

dependence of the integral area of the respective signal. The resulting graph is shown in Figure 6.23 .

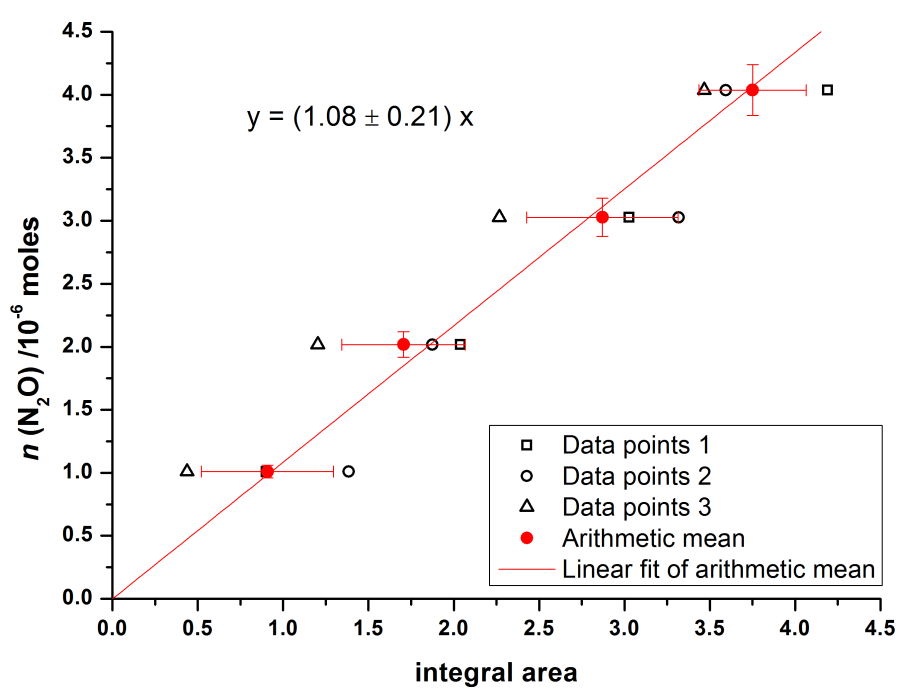

Figure 6.23: $\mathrm{N}_{2} \mathrm{O}$ calibration with $\mathrm{N}_{2} \mathrm{O}$ from a lecture bottle injected into the headspace via a Hamilton syringe. The black dots represent the collected data (three values for each volume of $\mathrm{N}_{2} \mathrm{O}$ ). The red dots indicate the arithmetic mean of these data sets. A linear dependence of the integral area of the $\mathrm{N}_{2} \mathrm{O}$ signals in the recorded IR spectra with the mole fraction of $\mathrm{N}_{2} \mathrm{O}$ molecules was assumed.

The arithmetic mean for three data points of each volume was generated and fitted linearly. Only volumes up to $100 \mu \mathrm{L}$ and thus four of the six data points were included in this fit. Above $100 \mu \mathrm{L}$ the data deviated strongly. The standard deviation, however, for the integral area was still very large (error bars in x direction). In this deviation errors such as inaccurate filling of the Hamilton syringe, small volume deviations or possible leaks of the gas IR cell were not included. For the molar concentration of $\mathrm{N}_{2} \mathrm{O}$ a deviation of $5 \%$ was assumed (error bars in y direction). However, in addition to the errors derived 
from the calibration, the quantification of $\mathrm{N}_{2} \mathrm{O}$ evolution featured further errors. Since very small amounts of $\mathbf{5}$ and $\mathrm{Cp}_{2} \mathrm{Co}$ were used to probe the evolution of $\mathrm{N}_{2} \mathrm{O}$, the mass error is substantial. Additionally problems were encountered in sealing the windows of the gas IR cell. Hence, it is possible that despite all efforts to gain comparable data the pressure within the cell prior to collecting the headspace deviated. Due to these problems and the rather large errors for the calibration curve, only an estimated range for the produced quantity of $\mathrm{N}_{2} \mathrm{O}$ can be given. For the above described four measurements of the headspace, conversions of $16-30 \%$ with respect to the complex concentration had to be accepted. The error for this measurement from the calibration alone ranges between 3 to $7 \%$. However, this error does not represent the major factor. The described setup problems probably extend the error margin significantly.

Assuming that these low yields of $\mathrm{N}_{2} \mathrm{O}$ can be attributed to problems with the setup and the rather large error margin and that aside from $5^{\text {Red }}$ no other side products form, a possible reduction scenario as described in Scheme 6.3 can be formulated.

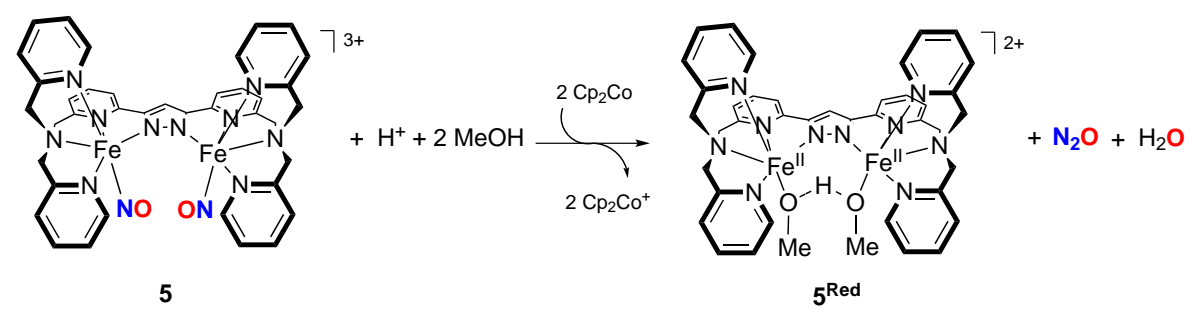

Scheme 6.3: Possible reduction sequence of $\mathbf{5}$.

In this reaction sequence one equivalent of $\mathbf{5}$ would react with two equivalents of $\mathrm{Cp}_{2} \mathrm{Co}$ and a proton to the already mentioned diiron(II) complex $\mathbf{5}^{\text {Red }}, \mathrm{N}_{2} \mathrm{O}$ and a water molecule. The proton source in this respect is probably the solvent methanol.

\subsubsection{Summary}

In this section the reduction of the two new diiron dinitrosyl complexes $\mathbf{4}$ and $\mathbf{5}$ was examined towards their behavior upon reduction by means of cyclic voltammetry and IR spectroscopy. The electrochemical reduction was additionally investigated by IR spectroelectrochemistry. Interestingly, the reaction pathways for the reduction of $\mathbf{4}$ and $\mathbf{5}$ differed. The reduction of $\mathbf{4}$ in $\mathrm{MeOH}$ resulted in the formation of the diiron(II) complex $\mathbf{4}^{\text {Red }}$ and a spectroscopically observed putative tetranitrosyl diiron complex (DNIC). $4^{\text {Red }}$ was furthermore isolated, crystallized and structurally characterized. In addition, Mössbauer and IR spectra were presented from isolated solid material after reduction and from bulk solution. Due to its poor solubility in many solvents no crystal structure of the putative tetranitrosyl diiron complex could be obtained to date, despite many attemps.

The reduction of $\mathbf{5}$ in $\mathrm{MeOH}$ did yield $\mathrm{N}_{2} \mathrm{O}$ upon reduction as previously postulated. $\mathrm{N}_{2} \mathrm{O}$ was identified and quantified via headspace IR gas analysis. The second reduction product was identified as the diiron(II) complex $\mathbf{5}^{\text {Red }}$, which was substantiated by structural analysis, Mössbauer and IR spectroscopy. Hence two different reaction pathways were demonstrated for the reduction of $\mathbf{4}$ and $\mathbf{5}$, which reflect the manifold reactivity of diiron dinitrosyl complexes. 
In comparison with the literature known model complexes XI (Lippard) and XII (Lehnert), the two new model systems $\mathbf{4}$ and $\mathbf{5}$ differ. Structurally $\mathbf{4}$ and $\mathbf{5}$ exhibit by $1 \AA$ longer $\mathrm{Fe} \cdots \mathrm{Fe}$ distances due to the diatomic pyrazolate bridge and rather stiff ligand backbone. However, as in XI and XII, also $\mathbf{4}$ and $\mathbf{5}$ feature a syn conformation of the two Fe-NO moieties. The $\mathrm{N} \cdots \mathrm{N}(\mathrm{Fe}-\mathrm{NO})$ distance is despite the significantly longer metal $\cdots$ metal distance only $0.1 \AA$ larger in $\mathbf{5}$ and $0.2 \AA$ larger in $\mathbf{4}$ compared to these model systems. The average $\mathrm{Fe}-\mathrm{N}-\mathrm{O}$ binding angles in $\mathbf{4}$ are slightly smaller compared to $\mathbf{5}$ and the two model systems. Since XI, XII and $\mathbf{5}$ have shown to produce $\mathrm{N}_{2} \mathrm{O}$, essential structural features, which these systems have in common, seem to lie specifically in the position of the two Fe-NO moieties with respect to each other within the bimetallic pocket. A close to planar syn conformation in this respect is highly beneficial. This might also explain the fact that $\mathbf{4}$ and the pyrazolate based diiron dinitrosyl complex XVIII by Kindermann, with a more tilted or in XVIII complete anti conformation do not produce $\mathrm{N}_{2} \mathrm{O}$ but choose different reduction pathways.

$\mathbf{5}$ moreover differs electronically from $\mathbf{4}$ and all diiron dinitrosyl model systems to date, as one Fe-NO moiety features a low spin state. How this property effects the reaction mechanism of $\mathbf{5}$ upon reduction could not be explained to date. 



\section{Excursus: Dinuclear Iron Complexes for Water Oxidation Catalysis}

Small molecules such as dihydrogen and dioxygen can be used as "green fuels" to generate energy through their controlled combustion. 9] However, to date these energy reservoirs are difficult to access as new ways have to be found to store these fuels in particular dihydrogen efficiently. [181] Nowadays the worlds energy supply is based $80 \%$ on fossil fuels, which dwindle significantly. $\frac{182}{}$ Aside from their gradual decline, the combustion of fossil fuels for energetic use generates greenhouse gases, mainly $\mathrm{CO}_{2}$ and further pollutants. 183 A primary goal in energy research involves finding alternative, more sustainable and recyclable ways to store and distribute energy. $[184$ Direct cleaner energy can be provided from solar panels, wind- or hydroelectric power plants. 184] On the downside this energy depends on many environmental factors and is directly attained in form of electricity, which can't easily be stored. A clean way to generate in the future efficiently storable "green" fuels is water splitting and/or the reduction of $\mathrm{CO}_{2}$. This process requires energy. The most abundant form of energy is solar energy. 185 . Hence strategies are needed to convert this energy into chemical fuels, for the storage of $\mathrm{H}_{2}$ and the controlled combustion of these fuels within the "knallgas" / oxyhydrogen reaction.

\section{Water splitting in Nature}

Nature realizes the water splitting process via photosystem II (PS II) in cyanobacteria, algae and higher plants with an efficiency of about $70 \%$. 186] Photosynthesis involves at least five steps for energy storage which are i) capture of sunlight, ii) charge separation, iii) catalysis iv) electron transfer to an electron acceptor $\left(\mathrm{NADP}^{+}\right)$and $\left.\mathrm{v}\right)$ assimilation of $\mathrm{CO}_{2}$ into carbohydrates. 187. Steps i) throughout iv) are considered as "light" reactions, whereas v) is a so-called "dark" reaction. A common way to describe the light driven steps in photosynthesis is the so-called Z-scheme (Scheme 7.1).

The transfer of electrons needed for the fixation and reduction of $\mathrm{CO}_{2}$ in photosynthesis occurs via a complex arrangement of redox active cofactors located in the photosystems $\mathbf{I}$ and II, together with a membrane complex $\left(\mathbf{C y t} \mathbf{b}_{6} \mathbf{f}\right)$. The initial charge separation and thus generation of electrons is realized by the oxygen evolving complex (OEC) in PS II, which drives the light dependent splitting of water into protons, electrons and dioxygen. 186 . 


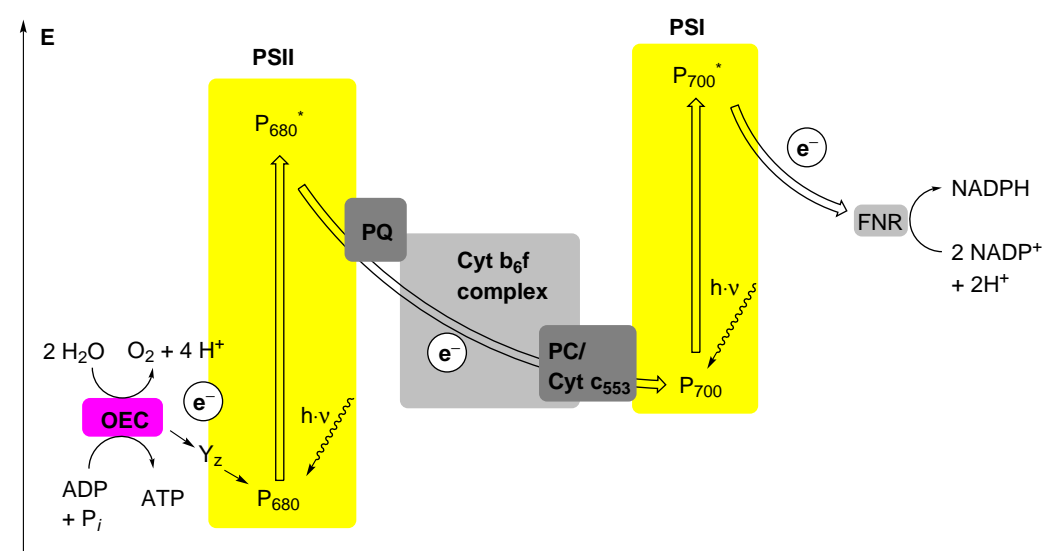

Scheme 7.1: Simplified Z-scheme. OEC: oxygen evolving complex, $\mathbf{Y}_{Z}$ : redox active tyrosine, $\mathbf{P}_{680} / \mathbf{P}_{700}$ : chlorophyll $\mathbf{P}_{680} / \mathbf{P}_{700}, \mathbf{P Q}$ : plastoquinone complex, $\mathbf{C y t} \mathbf{b}_{6} \mathbf{f}$ : cytochrome $b_{6}$ f membrane complex, PC: plastocyanin, FNR: ferredoxin-NADP ${ }^{+}$oxidoreductase.

$$
2 \mathrm{H}_{2} \mathrm{O} \longrightarrow \mathrm{O}_{2}+4 \mathrm{H}^{+}+4 \mathrm{e}^{-}
$$

For this step the OEC needs to absorb four photons, which are collected by an array of chlorophylls in light harvesting proteins and are funneled to the photoactive reaction center $\mathbf{P}_{680}$ in PSII. 188 Upon excitation of $\mathbf{P}_{680}$ a radical cation is generated. The free electron is accepted by a pheophytin molecule and passed along the electron transfer chain via two plastoquinones (PQ), the electron transfer complex $\mathbf{C y t} \mathbf{b}_{6} \mathbf{f}$ and a plastocyanin to PS I. The charge separated excited state $\mathbf{P}_{680}{ }^{*}$ is stabilized by a redox active tyrosine $\left(\mathbf{Y}_{Z}\right)$. When $\mathbf{P}_{680}{ }^{*}$ is rereduced by $\mathbf{Y}_{Z}$, the tyrosyl radical $\mathbf{Y}_{Z}^{*}$ is formed. Reduction of $\mathbf{Y}_{Z}$ is coupled to the $\mathbf{O E C}$. As the $\mathbf{O E C}$ mediates the water splitting reaction which is a four electron reduction, the nearby tyrosine $\left(\mathbf{Y}_{\mathrm{Z}}\right)$ has to be singly oxidized in four cycles by $\mathbf{P}_{680}{ }^{*}$ before one equivalent of dioxygen is produced. Aside from the electron transfer chain also protons are transferred, thus the net reactions are proton coupled electron transfer processes (PCETs). 187/188

PS I is the second light driven electron transfer center in photosynthesis. Here the electron transfer chain yields in a ferredoxin-NADP ${ }^{+}$oxidoreductase $($FNR), which requires electrons to generate the "reduced hydrogen carrier" $\mathrm{NADPH}$ needed for $\mathrm{CO}_{2}$ fixation. [186]

The OEC is an excellent model to study homogeneous water oxidation catalysis. The complex exhibits a $\mathrm{Mn}_{4} \mathrm{O}_{5} \mathrm{Ca}$ structural core motif, in which three manganese- and one calcium atom form a cubane like structure with four oxo ligands. A fourth dangling manganese atom is attached via an oxo bridge to one plane of the cubane. The $\mathrm{Mn}_{4} \mathrm{O}_{5} \mathrm{Ca}$ cluster is moreover surrounded by several amino acid residues and water molecules. 189 .

The mechanism of how this cluster masters the four electron reduction and water splitting reaction is part of ongoing research. The current consensus states that starting from a $\mathrm{Mn}^{\mathrm{III}} \mathrm{Mn}_{3}^{\mathrm{IV}}$ species the cluster is sequentially oxidized to a $\mathrm{Mn}_{4}^{\mathrm{IV}}$ species. The fourth oxidation might occur either metal or ligand centered by generating a high valent $\mathrm{Mn}^{\mathrm{V}}$ metal center or a $\mathrm{Mn}^{\mathrm{IV}}$-oxyl radical which might facilitate $\mathrm{O}$... O bond formation. 190/191] In the course of these oxidation steps the cluster appears to undergo significant core rear- 


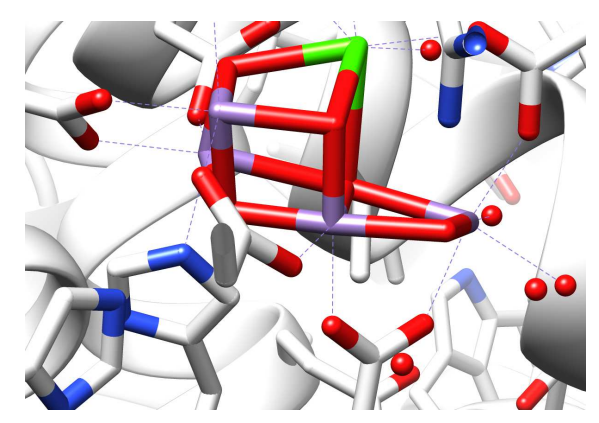

Figure 7.1: $\mathrm{Mn}_{4} \mathrm{O}_{5} \mathrm{Ca}$ cluster of the oxygen evolving complex OEC in a $1.9 \AA$ resolution (PDB entry: 3ARC). 189 .

rangement processes. 191

\section{Homogeneous Water Oxidation Catalysis}

On a laboratory scale the water splitting reaction can nowadays be realized by a variety of materials. Aside from heterogeneous catalysts, i.e., nanoparticles and metal oxides, a large library of homogeneous catalysts has been prepared. These catalysts largely contribute to fundamental research in elucidating the reaction sequence of the splitting reaction and by optimizing reaction conditions. The successful development of a water oxidation catalyst is based on many factors, among others i) a rational design based on ligand modifications, choice of transition metal, geometry, electronic coupling and through space interactions, ii) good solubility in water and iii) a possibility to anchor the catalyst onto electrodes. 184 The best characterized water oxidation catalysts in this field rely to date on the elements manganese, ruthenium and iridium. ${ }^{192}$ Manganese complexes are prepared as they exhibit a close resemblance to the OEC, while the use of the $4 \mathrm{~d}$ and $5 \mathrm{~d}$ transition metals ruthenium and iridium is owed to the fact that stable complexes in high oxidation states are accessible with these metal ions. 192/193] A growing interest over the last couple of years can be observed in the increased use of more abundant and cheaper 3d metals. 193.

Iron complexes have been considered as water oxidation catalysts only in the last few years. This is attributed to the fact that their catalytic mechanisms and degradation pathways are not well understood to date. 193 . Common problems often involve the lack of stability of iron complexes, which leads to very low lifetimes and fast decomposition. 194 Another key problem lies in the solubility of iron complexes with organic ligand frameworks in aqueous media. 194

A few iron water oxidation catalysts (WOCs) reported recently which showed promising results in stability and efficiency shall be mentioned in this context. Iron complexes with tetraamido macrocyclic ligands (TAML), initially developed for the activation of dioxygen and peroxides 195]196 have recently revealed water oxidation activity. 197. The experiments were conducted under standard conditions which are usually applied for nobel metals using $\mathrm{Ce}^{\mathrm{IV}}$ as oxidant at $\mathrm{pH}=1$. However, doubt is casted on the reliability that the Fe-TAML species is the active catalyst as TAML ligands are known to undergo demetalation under acidic conditions. Hence, the active species would be attributed to iron 
oxide nanoparticles. $198 \mid 199$ Latest modifications of the ligand design and/or the use of different more alkaline reaction conditions yielded more reliable results for efficient water oxidation catalysis mediated by TAML iron complexes. ${ }^{200}$ Mechanistically a nucleophilic attack of water onto a $\mathrm{Fe}^{\mathrm{V}}(\mathrm{O})$ leading to the formation of a $\mathrm{Fe}^{\mathrm{III}}$ - hydroperoxo complex has been proposed. This intermediate would liberate $\mathrm{O}_{2}$ under oxidative conditions. [200] Two more systems noteworthy of mentioning are the mononuclear catalysts developed by the Lloret-Fillol/Costas group (Scheme 7.2). Their ligand design features iron sites coordinated by four nitrogen donor atoms and two labile coligands in cis position. Under standard water oxidation conditions with $\mathrm{Ce}^{\mathrm{IV}}$ these iron WOCs form a spectroscopically detectable iron-oxo-cerium intermediate prior to $\mathrm{O}-\mathrm{O}$ bond formation. 201.

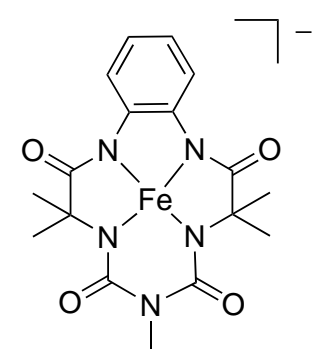

Biuret modified Fe(TAML) Panda 2014

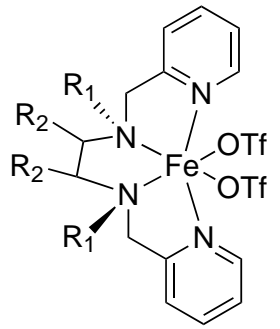

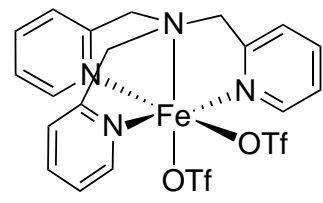

Non heme Fe catalysts Lloret-Fillol/Costas 2011

Scheme 7.2: Iron WOCs by Ellis/Panda and Lloret-Fillol/Costas.

\section{Dinuclear Iron WOCs}

Diiron catalysts have not been studied very well in the context of water oxidation catalysis. 131 A few examples have been presented with diiron oxo bridged complexes, using well characterized tridentate trispyridylmethylamine ligands [131|202] or with tetradentate polypyridyl-type ligands by Thummel et al. 203 However, this field of research is still in its infancy and not very well understood.

The design of new diiron catalysts is nevertheless very promising as with two metal centers in analogy to many noble metal diruthenium or diiridium complexes, the four necessary electrons for water oxidation catalysis can be provided. As discussed before many non heme diiron enzymes in nature make use of the metal's rich redox chemistry for small molecule activation. Hence water oxidation with diiron complexes should also lead to respectable results in the future.

In this chapter the bis(pentadendate) ligand scaffold $\mathbf{K}_{5} \mathbf{L}^{\mathbf{C O O}}$ is employed to develop new dinuclear iron water oxidation catalysts. The carboxylate moieties of the ligand scaffold are anticipated to provide resulting complexes with a reasonably good solubility in water. Moreover chelation of two metals by the ligand scaffold should provide stability in neutral and basic media. The strong anionic character of $\mathbf{L}^{\mathbf{C O O}}$ should in addition be well suitable for the stabilization of high oxidation states during catalysis. 


\subsection{A First Dinuclear Iron Complex with the Ligand L $\mathrm{COO}$}

\section{Synthesis and Structural Characterization}

The ligand $\mathbf{L}^{\mathbf{C O O}}$ comprises a fivefold negatively charged ligand scaffold. When incorporating iron ions into the two binding pockets, a $\left\{\mathrm{N}_{3} \mathrm{O}_{2}\right\}$ binding motif for each metal can be provided by the ligand alone. To probe whether dinuclear iron complexes would form in the desired way, the ligand was dissolved in the aprotic but polar solvent dimethylformamide (DMF). As iron salt $\mathrm{Fe}\left(\mathrm{BF}_{4}\right)_{2} \cdot 7 \mathrm{H}_{2} \mathrm{O}$ was chosen. Other than in previous synthetic routes no base was needed to deprotonate the ligand since the potassium salt of the ligand was used. A color change from colorless to orange was observed upon mixing ligand and iron salt. Diffusion of diethyl ether into this reaction mixture afforded orange block shaped single crystals. When mounting these crystals on a sample holder for x-ray diffraction and measuring at $133 \mathrm{~K}$, a decreasing quality of the diffraction pattern was observed. For this reason only a picture which reflects atom connectivities in this complex (6) was attained (Figure 7.2).

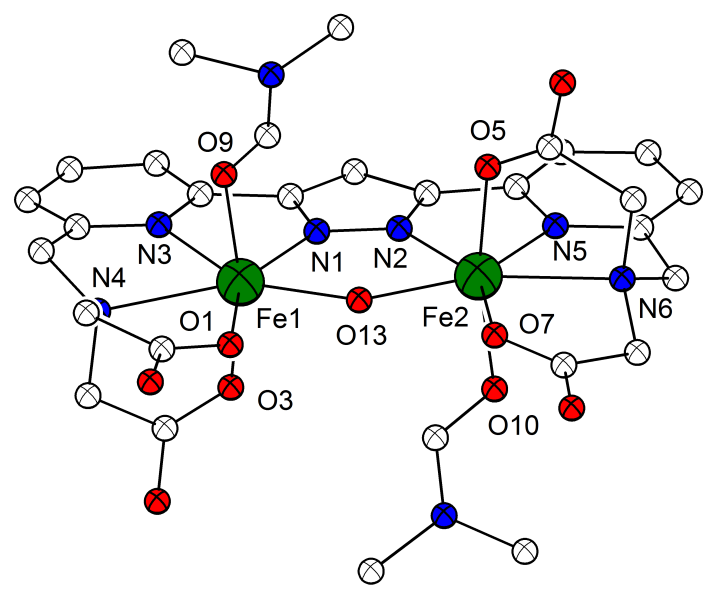

Figure 7.2: Picture of $\left[\{\mathrm{Fe}(\mathrm{DMF})\}_{2}(\mu-\mathrm{OH})\right] 6$ in a "ball and stick" representation.

Due to the poor quality of the measurement, a representation with thermal ellipsoids was not possible. Hence the radii of all atoms were fixed. 6 comprises a dinuclear iron core with a monoanionic hydroxo bridge. Additionally, each iron atom features one axial solvent (DMF) ligand. The complex itself is neutral in charge. The two metal ions on the contrary hold a charge of + III each. The two solvent ligands oppose each other in an anti fashion and coordinate via the oxygen atom of the DMF molecule.

$\mathrm{ESI}^{-}$mass spectra of 6 in $\mathrm{MeOH}$ display an oxo bridged complex without the coligand DMF. Thus the ligand system $\mathbf{L}^{\mathbf{C O O}}$ seems to be more flexible compared to the complexes with nitrogen side arms presented in this work (compared to 3). Moreover the coligands seem to be labile and/or exchangeable at least under the ionizing conditions of ESI mass spectrometry. 


\section{Electrochemistry}

Cyclic and square wave voltammograms of $\mathbf{6}$ were recorded in oxygen free dimethylformamide (DMF) and in the polar protic solvent methanol. As internal reference ferrocene was added. Figure 7.3 depicts the respective voltammograms in methanol as methanol is more similar to the target solvent water. Two reduction events can be observed. These can be tentatively assigned to two sequential reduction events from $\mathrm{Fe}(\mathrm{III}) \mathrm{Fe}(\mathrm{III})$ to $\mathrm{Fe}$ (II)Fe(II). However, the first reduction is not very well resolved and can hardly be described as reversible due to the large separation of the cathodic and anodic peak. The two redox potentials $\mathrm{E}_{1 / 2}$ from square wave voltammetry lie at $-0.35 \mathrm{~V}$ vs. $\mathrm{Fc} / \mathrm{Fc}^{+}$(III,III/III,II) and $-0.75 \mathrm{~V}$ vs. $\mathrm{Fc} / \mathrm{Fc}^{+}$(III,II/II,II), respectively.

If the entire solvent window is scanned (Figure 7.3 right), it becomes apparent that no oxidation events can be observed. The cyclic voltammograms of $\mathbf{6}$ and the recorded solvent background match very well above $0 \mathrm{~V}$ vs. $\mathrm{Fc} / \mathrm{Fc}^{+}$. Hence $\mathbf{6}$ exhibits a great stability in methanol and cannot be oxidized within the recorded range to higher oxidation states.
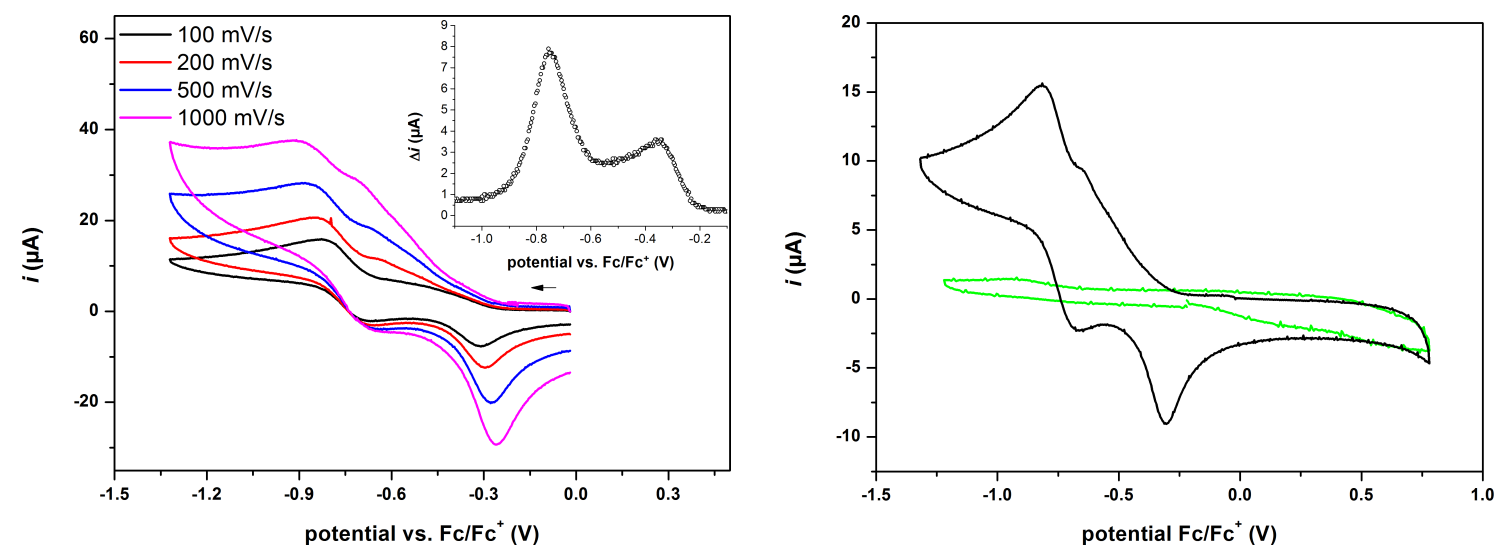

Figure 7.3: Cyclic voltammogram of 6 in $\mathrm{MeOH}\left(0.1 \mathrm{M} \mathrm{NBu} 4 \mathrm{PF}_{6}\right)$. Left: Scan rate dependent cathodic reduction. Right: Full voltammogram (black line) compared to background (green line). No significant oxidation events can be observed in the measured range.

The redox potentials of $\mathbf{6}$ in methanol for the $\mathrm{Fe}(\mathrm{II}) \mathrm{Fe}(\mathrm{III})$ couples lie at lower potentials compared to diruthenium complexes. 119]121] Hence the redox chemistry of diiron complexes differs significantly from the chemistry of noble metals. Possibly their utilization in water oxidation catalysis requires new approaches and different conditions compared to conventional methods.

\subsection{A Water Soluble Diiron Diaqua Complex}

\section{Synthesis and Structural Characterization}

As ligand and iron salt used for the synthesis of $\mathbf{6}$ are soluble in demineralized water, attempts were made to prepare a "water based" dinuclear iron complex. When dissolving $\mathbf{K}_{5} \mathbf{L}^{\mathbf{C O O}}$ and $\mathrm{Fe}\left(\mathrm{BF}_{4}\right)_{2} \cdot 7 \mathrm{H}_{2} \mathrm{O}$ in water a red solution is obtained. This solution was stirred for a few minutes and quickly filtered. Diffusion of acetone into the reaction mixture afforded red single crystals suitable for x-ray diffraction. The obtained neutral 
complex $\mathbf{7}$ crystallizes in the triclinic space group $P \overline{1}$ with one complex molecule, $5.5 \mathrm{H}_{2} \mathrm{O}$ molecules and one acetone molecule in its unit cell. The structure of $\mathbf{7}$ features two sevencoordinate iron sites bridged by a hydroxo ligand. A $\left\{\mathrm{N}_{3} \mathrm{O}_{4}\right\}$ donor set can be assigned for each metal. Aside from the chelating ligand and the hydroxo bridge, water molecules coordinate to both iron sites. Interestingly, these two water molecules point towards the same side of the complex in a syn fashion. The Fe $\cdots$ Fe distance in 7 is $3.75 \AA$, which is fairly short compared to the corresponding diiron complexes in this work with entirely nitrogen based ligands. Figure 7.4 displays the molecular structure of the neutral complex 7.

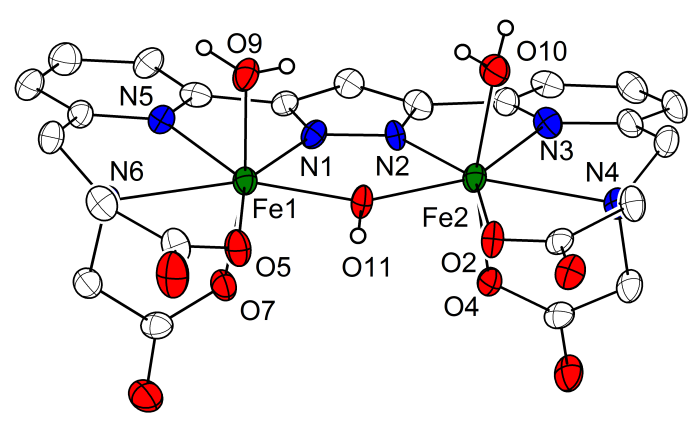

Figure 7.4: Crystal structure of $\left[\mathrm{L}^{\mathrm{COO}}\left\{\mathrm{Fe}\left(\mathrm{H}_{2} \mathrm{O}\right)\right\}_{2}(\mu-\mathrm{OH})\right],\left(\mathrm{CH}_{3}\right)_{2} \mathrm{CO}, 5.5 \mathrm{H}_{2} \mathrm{O}$ 7. Thermal ellipsoids are set to $50 \%$. Not relevant hydrogen atoms as well as solvent molecules were omitted for clarity. Selected bond lengths $[\AA]$ and angles $\left[{ }^{\circ}\right]$ Fe1-N1 2.089(4), Fe1-N5 2.315(4), Fe1-N6 2.344(4), Fe1-O5 2.126(4), Fe1-O7 1.993(3), Fe1-O9 2.015(4), Fe1-O11 2.004(3), Fe2-N2 2.089(4), Fe2-N3 2.308(4), Fe2-N4 2.385(4), Fe2-O2 2.106(4), Fe2-O4 1.986(3), Fe2-O10 2.012(4), Fe2-O11 1.997(3), Fe2-O11-Fe1 139.65(19), Fe1-N1-N2-Fe2 -3.166(6).

Since the ligand is fivefold negatively charged and the hydroxo bridge is a monoanionic ligand a +III charge results for each iron atom.

As shortly addressed before the two water ligands in 7 coordinate to the metal centers in a syn fashion. This can be seen as excellent preorganisation of the substrate water for the catalysis. Generally two water oxidation mechanisms are described for dinuclear water oxidation catalysts in literature. $\frac{125}{}$ After the oxidation of the bimetallic complex, two metal oxo units preferably in syn orientation are formed.

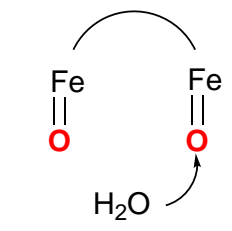

Water Nucleophilic Attack (WNA)

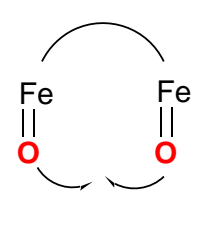

Interaction of Two M-O Units (I2M)

Scheme 7.3: Discussed mechanisms of $\mathrm{O}-\mathrm{O}$ bond formation in water oxidation catalysis.

For $\mathrm{O}-\mathrm{O}$ bond formation a water molecule attacks as a nucleophile at one of the metal- 
oxo units to yield a hydroperoxo and a hydroxo ligand. A second water molecule then liberates dioxygen and the initial two water molecules coordinating to the dinuclear core are regained. This mechanism is called water nucleophilic attack (WNA). A second option involves $\mathrm{O}-\mathrm{O}$ bond formation via a direct interaction of two metal oxo units (I2M). 125] However, for both reaction pathways $\mathbf{7}$ might be an appropriate precursor due to its syn water coordination.

$\mathrm{ESI}^{-}$mass spectrometry from a mixed water/isopropanol solution reveals as observed for 6, a "naked" oxo-bridged diiron complex without any coligands (Appendix 11.5. Figure 11.20. The main signal in $\mathrm{ESI}^{+}$spectra can be attributed to the hydroxobridged complex without the water coligands together with a sodium cation.

\section{Mössbauer Spectroscopy}

To determine the spin state of the two iron sites in 7, Mössbauer measurements were conducted. In this respect diiron complexes differ greatly from nobel metal complexes as these feature mostly low spin configurations. Among the $3 \mathrm{~d}$ metals however, due to smaller ligand field splittings the spin state of a metal in coordination complexes strongly depends on the coordination sphere and nature of the donor sites. Zerofield Mössbauer spectroscopy of a polycrystalline sample of 7 at $80 \mathrm{~K}$ suggests a single iron(III) species which accounts for both iron atoms, as both feature the same coordination geometry. The isomer shift of $\delta=0.57 \mathrm{~mm} \mathrm{~s}^{-1}$ and the rather small quadrupole splitting point towards a largely spherical electron distribution and thus a Fe(III) high spin state. $\frac{204}{2}$ Figure 7.5 illustrates the recorded spectrum of $\mathbf{7}$.

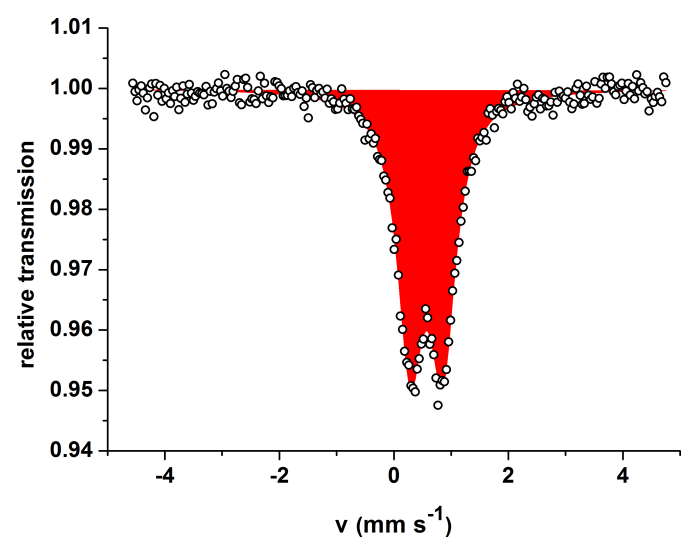

Figure 7.5: Zerofield Mössbauer spectrum of a polycrystalline sample of $\mathbf{7}$ at $80 \mathrm{~K}$. Isomer shift $\delta$ and quadrupole splitting $\left|\Delta E_{\mathrm{Q}}\right|$ in $\left[\mathrm{mm} \mathrm{s}^{-1}\right]: \delta=0.57,\left|\Delta E_{\mathrm{Q}}\right|=0.57$.

High spin complexes usually exhibit different electronic properties compared to low spin complexes which express themselves in profound changes in optical, vibrational, magnetic and structural properties. $\underline{4}$

\section{Electrochemistry}

In analogy to $\mathbf{6}$, the redox potentials of $\mathbf{7}$ were examined by means of cyclic voltammetry. A polycrystalline sample of $\mathbf{7}$ was dissolved in an aqueous solution $\left(0.1 \mathrm{M} \mathrm{NaClO}_{4}\right)$. As 
working and counterelectrode a glassy carbon and platinum electrode were employed. A saturated calomel electrode (SCE) was used as reference electrode. The respective measurements are shown in Figure 7.6
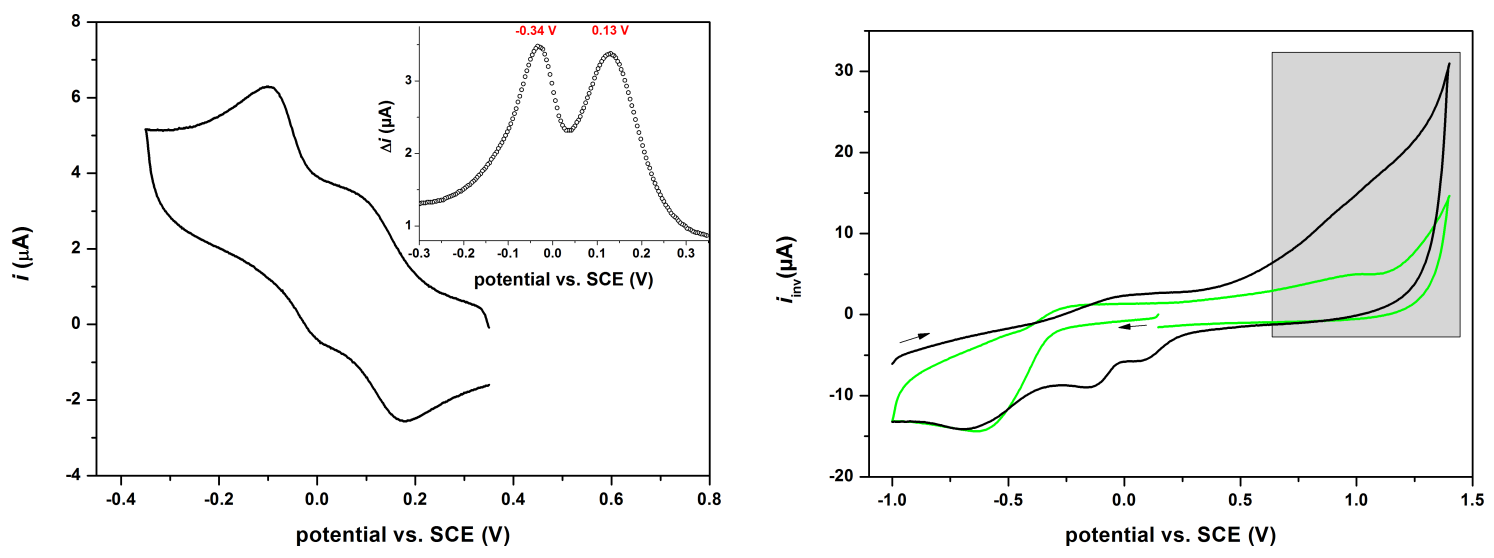

Figure 7.6: Left: Cyclic voltammogram of 7 in neutral $\mathrm{H}_{2} \mathrm{O}\left(0.1 \mathrm{M} \mathrm{NaClO}_{4}\right)$ vs SCE reference. The inset shows the corresponding square wave diagram. Right: Large window view. Currents were inverted for a better comparison with CV's according to the water oxidation formalism. The green trace describes the solvent background. The cyclic voltammogram of $\mathbf{7}$ is depicted in black. The grey box highlights the range in which water oxidation might occur.

The cyclic voltammogram of $\mathbf{7}$ exhibits as previously described for $\mathbf{6}$ two reduction events marking the $\mathrm{Fe}$ (II)Fe(III) couples. The two redox potentials $\mathrm{E}_{1 / 2}$ from square wave voltammetry lie at $0.13 \mathrm{~V}$ vs. SCE (III,III/III,II) and $-0.34 \mathrm{~V}$ vs. SCE (III,II/II,II), respectively. Zooming out of the cathodic reduction range, into a large window view (Figure 7.6 right) an enhanced current can be observed above $0.5 \mathrm{~V}$ vs. SCE. However, this oxidation event does not resemble the typical plateau shape of electrocatalytic events as observed for wellstudied water oxidation catalysts. Generally, a more accurate prediction could be made with additional measurements at different scan rates for potentials above $0.5 \mathrm{~V}$ vs. SCE. Simple electrocatalytic events exhibit an S-shaped curve that is independent of the scan rate and can be described by the following equation: 205

$$
i=\frac{i_{\mathrm{pl}}}{1+\mathrm{e}^{\frac{\mathrm{F}}{\mathrm{RT}}\left(E-E_{1 / 2}\right)}}
$$

$i \quad$ current

$i_{\mathrm{pl}} \quad$ current at the plateau

$E \quad$ electrode potential

$E_{1 / 2}$ half-wave potential of the steady-state catalytic wave

A plateau shaped current was not observed. No scan rate dependent measurements were performed as problems were encountered with the solubility of the complex in neutral water. Between each CV cycle vigorous stirring of the solution ensured a maximum solvation of the sample. However, still slight solid sample residues were observed during the measurement. The inhomogeneity of the solution can have basically two most probable reasons. One is that $\mathbf{7}$ is simply poorly soluble in neutral water, but stable over the course of the measurement. Another reason could be that over the course of the measurement iron oxide nanoparticles are produced, which leads to depletion. A variety of other events 
that might contribute to inhomogeneity are certainly also possible. 206 A comprehensive experiment to rule out the formation of nanoparticles would involve a rinse test in which a CV with the same electrodes but a fresh solution without sample would be recorded. If metal oxide particles formed on the surface of the electrodes, enhanced currents would be observed. Another possibility could involve a blank test, in which simply a CV of the iron salt is recorded. However, a more thorough electrochemical analysis of $\mathbf{7}$ was beyond the scope of this work.

To probe whether 7 performs water oxidation under the well studied common water oxidation conditions in triflic acid $(\mathrm{pH}=1)$ and with $\left(\mathrm{NH}_{4}\right)_{2}\left[\mathrm{Ce}^{\mathrm{IV}}\left(\mathrm{NO}_{3}\right)_{6}\right](\mathrm{CAN})$ as oxidant the oxygen evolution was measured with a Clarke electrode. Apparently $\mathbf{7}$ is not very well soluble at low $\mathrm{pH}$ values. The oxidant was added anyway to a suspension of $\mathbf{7}$ in triflic acid. A color change from a red suspension to a yellow solution with white precipitate was observed in the course of minutes. Additionally, no oxygen evolution was detected. Consequently, it can be assumed that these standard conditions are not suitable for the oxidation of $\mathbf{7}$. The complex decomposed. ESI mass spectra of the compound after oxidation did not contain any signals typical for the diiron complex.

It seems that the oxidizing strength of $\mathrm{Ce}^{\mathrm{IV}}$ is too large for the oxidation of 7 . The redox potential of CAN of $1.75 \mathrm{~V}$ vs. $\mathrm{NHE}$ (at $\mathrm{pH}=0.9$ ) is very high. 207. The observed oxidation event of 7 in neutral water features an onset of about $0.5 \mathrm{~V}$ vs. SCE which is significantly lower. Maybe the choice of a milder oxidant would be wise in this context. Further well established oxidants for water oxidation catalysis are $\left[\mathrm{Ru}{ }^{\mathrm{III}}(\mathrm{bpy})_{3}\right]^{3+}(1.21 \mathrm{~V}$ vs. NHE), sodium peroxodisulfate ( $2 \mathrm{~V}$ vs NHE), Oxone $(1.82 \mathrm{~V}$ vs. NHE) and sodium periodate $\left(1.6 \mathrm{~V}\right.$ vs. NHE). ${ }^{207}$ The only oxidant of these reported ones, $\left[\mathrm{Ru}{ }^{\mathrm{III}}(\mathrm{bpy})_{3}\right]^{3+}$, which would be milder, requires similar to $\mathrm{Ce}^{\mathrm{IV}}$ acidic conditions, in which $\mathbf{7}$, as shortly addressed above, is only poorly soluble. Moreover, the modification of $\mathbf{7}$ at low $\mathrm{pH}$ values is unknown. In the future, a speciation analysis would be helpful. Also excluded should be the possibility of complex degradation in acidic media. In that respect it would be necessary to probe the complex stability of $\mathbf{7}$ within a longer time window in respective acids. However, again the poor solubility of $\mathbf{7}$ did not allow for a detailed analysis by spectroscopic monitoring.

\section{pH-Titration followed by UV/Vis Spectroscopy}

Since cyclic voltammtry revealed solubility problems of $\mathbf{7}$ in acidic and neutral aqueous solutions a pH-titration was performed followed by UV/Vis spectroscopy. For this measurement a definite amount of polycrystalline 7 was dissolved in a solution of $0.1 \mathrm{M} \mathrm{HCl}$ and $0.1 \mathrm{M} \mathrm{KCl}$. This mixture was titrated with defined amounts of $0.1 \mathrm{M} \mathrm{NaOH}$ and the $\mathrm{pH}$ was recorded. $\mathrm{KCl}$ was used to maintain a close to constant ionic strength during the measurement. For every $\mathrm{pH}$ value a UV/Vis spectrum was measured. The corresponding spectra are depicted in Figure 7.7 .

For $\mathrm{pH}$ values below $\mathrm{pH}=7$ two absorption maxima at 243 and $290 \mathrm{~nm}$ can be observed for 7 . The intensity of these absorption bands increases with increasing $\mathrm{pH}$ value. Above $\mathrm{pH}=7$ the bands shift about $12 \mathrm{~nm}$ to larger wavelengths. A general observation is more- 


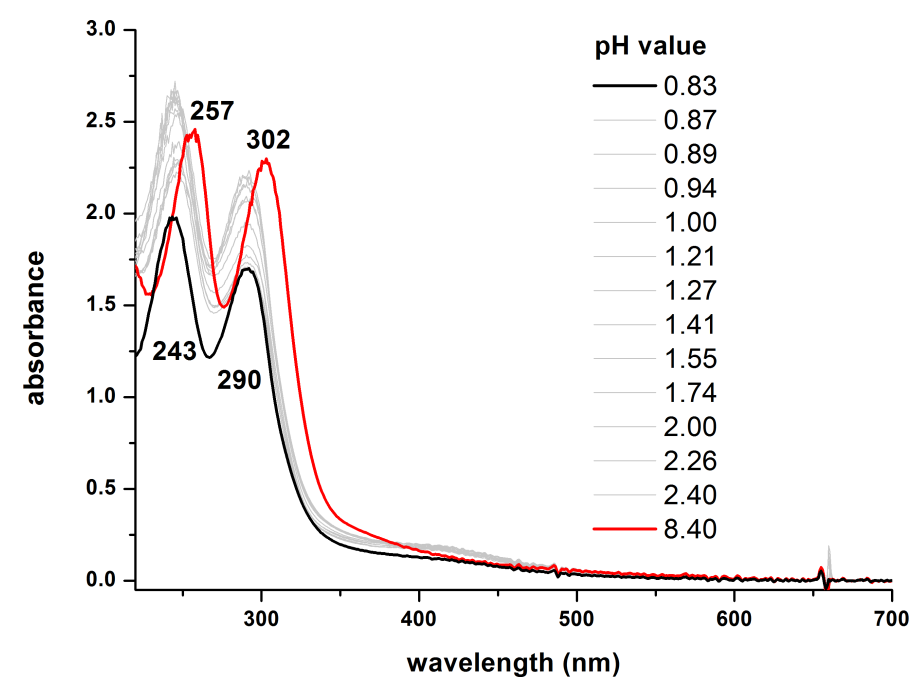

Figure 7.7: pH dependent UV/Vis titration of 7. The black line represents the initial spectrum at low $\mathrm{pH}$ values, the red line depicts a spectrum under basic conditions.

over that 7 dissolves well in basic solution, which also explains the increasing intensity.

This suggest that in future experiments water oxidation catalysis with $\mathbf{7}$ should be best performed in buffer solutions to maintain a slightly basic $\mathrm{pH}$, which ensures full solubility of the complex. A suitable non coordinating buffer that fulfills this requirement has not been found yet. However, a buffer is inevitably important to perform these experiments as at lower $\mathrm{pH}$ values iron oxide nanoparticles might form, which can also take part in catalytic events and thus tamper with the entire experiment.

\subsection{Analysis and Electrochemistry of a Tetranuclear Manganese Complex}

\section{Synthesis and Structural Characterization}

In support of previous experiments the ligand $\mathbf{K}_{5} \mathbf{L}^{\mathbf{C O O}}$ was once more reacted with manganese acetate in a 1:1 mixture of methanol and water. As the OEC and quite a few model systems are based on manganese ions and the metal exhibits a rich redox chemistry this might lead to promising results. Crystals could be obtained by diethyl ether diffusion into the reaction mixture. However, the quality of these crystals was very poor, so that from x-ray diffraction techniques only a picture is presentable. The complex 8 crystallizes as the tetranuclear twofold negatively charged complex anion $\left[\mathrm{L}_{2}^{C O O} \mathrm{Mn}_{4}\right]^{2-}$. Two potassium cations compensate this charge. The complex anion of $\mathbf{8}$ is depicted in Figure 7.8 .

All manganese ions in $\mathbf{8}$ are twofold positively charged. Interestingly, one carboxylic side arm of each dimanganese unit forms a bridge to the respective other dimanganese unit. Hence, a tetranuclear cluster results. This structural motif is in so far relevant in particular for water oxidation catalysis as also the OEC in nature mediates water splitting via a tetranuclear manganese cluster. 


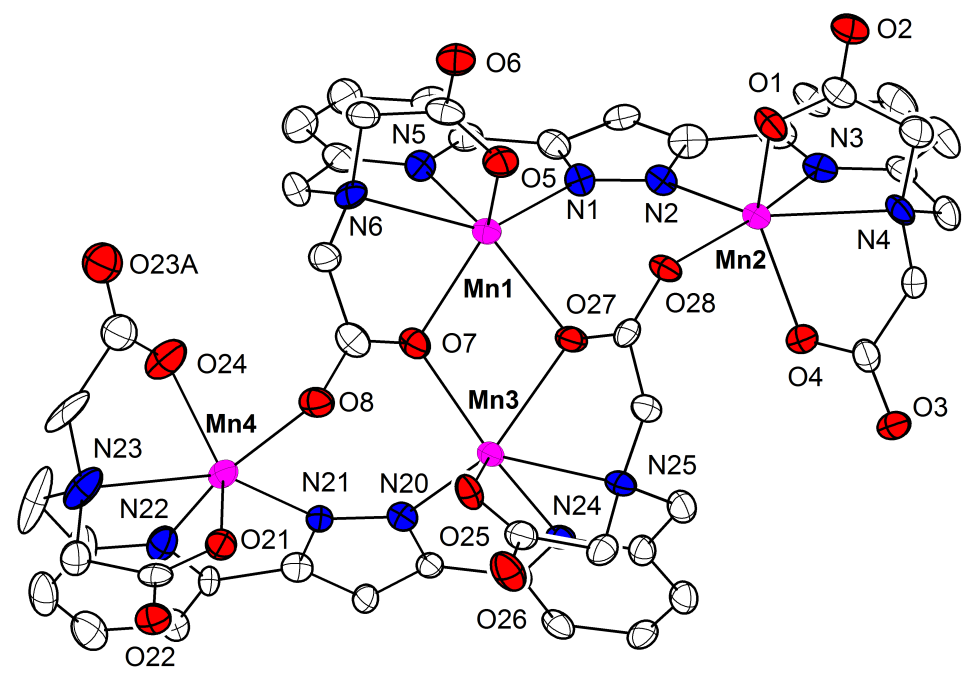

Figure 7.8: Picture of the complex anion of $\mathrm{K}_{2}\left[\mathrm{~L}_{2}^{\mathrm{COO}} \mathrm{Mn}_{4}\right]$, $\mathrm{xMeOH}, \mathrm{xH}_{2} \mathrm{O}$ (8). Thermal ellipsoids are set to $50 \%$. Not relevant hydrogen atoms as well as solvent molecules were omitted for clarity.

\section{Mass Spectrometry and Magnetic Susceptibility Measurements}

8 was additionally characterized via ESI mass spectrometry. Since the complex anion is negatively charged primarily an $\mathrm{ESI}^{-}$mass spectrum was recorded. Two main signals can be observed in the spectrum at $m / z=564$ and 617 (Figure 7.9).

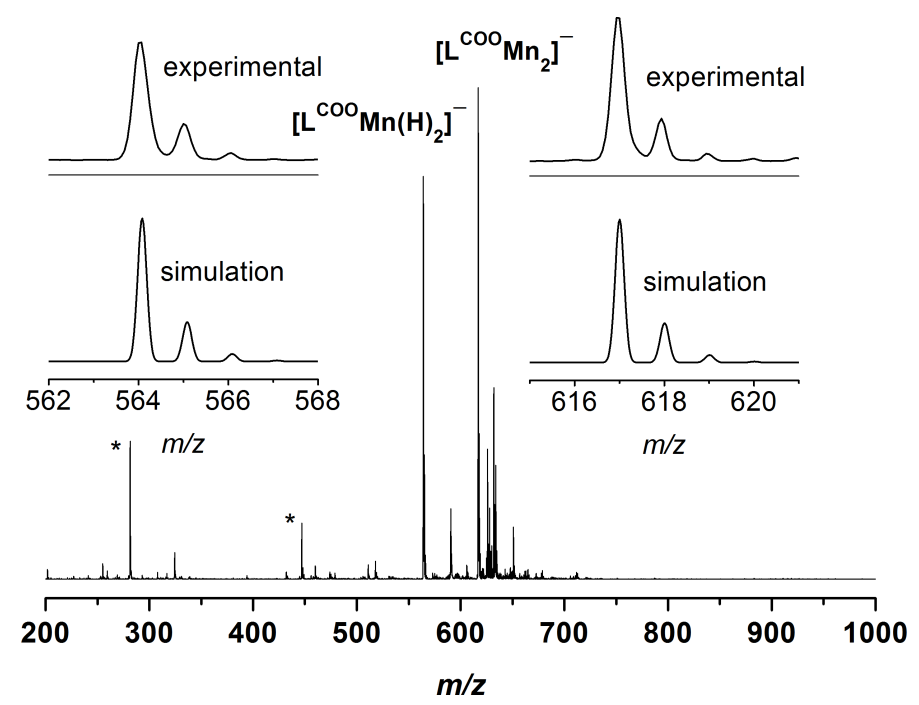

Figure 7.9: $\mathrm{ESI}^{-}$mass spectrum of 8 from a $\mathrm{MeOH} / \mathrm{H}_{2} \mathrm{O}$ mixture 1:1. The asterisk marks signals which stem from the spectrometer and/or solvent impurities and hence can't be assigned to the sample.

These two signals can be assigned to a mononuclear manganese species $\left[\mathrm{L}^{\mathrm{COO}} \mathrm{Mn}\left(\mathrm{H}_{2}\right)\right]^{-}$ and the dinclear complex $\left[\mathrm{L}^{\mathrm{COO}} \mathrm{Mn}_{2}\right]^{-}$. Hence, under ionizing conditions in aqueous solution the tetranuclear structure of $\mathbf{8}$ fragments into two dinuclear manganese complexes. An additional signal for the tetranuclear complex is not present in the recorded spectra. 
Moreover, the carboxylate functions of the side arm seem to protonate easily at least under ionizing conditions. As a result one metal site is removed from of the dinuclear complex resulting in the observed mononuclear species.

Consequently, solutions of $\mathbf{8}$ contain several species. However, from mass spectrometry it is not possible to assign which species dominates in solution.

To determine the spin states of the four manganese atoms in solid state, magnetic susceptibility measurements were conducted. The sample was prepared according to general procedures, in which the polycrystalline material is placed inside a gel capsule, which is mounted inside a sample holder (PTFE straw). Figure 7.10 depicts the plot of the product of magnetic susceptibility and temperature vs. the temperature in a range of 2 to $300 \mathrm{~K}$. The aquired data were fit with respect to the following assumptions. All g-values were set to 2.0. A diamagnetic impurity of $6.5 \%$ with a spin of $S=0$ was included as parameter and at low temperatures an antiferromagnetic coupling of Mn1 and Mn3 via the monoatomic oxygen bridge was considered. Moreover, all four manganese atoms featured a $S=5 / 2$ spin state.

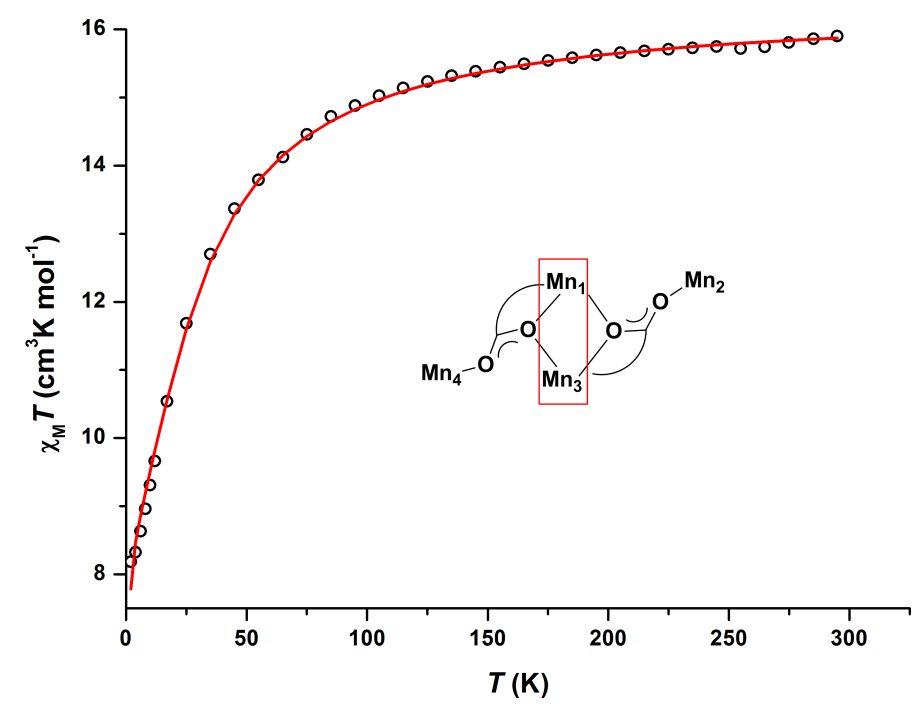

Figure 7.10: Magnetic susceptibility measurement of a polycrystalline sample of 8 (black circles). Data were fit (red line) assuming a spin state of $\mathrm{S}=5 / 2$ for each manganese atom. A paramagnetic impurity of $6.5 \%$ was included in the fit. The $g$-values were all set to 2.00 and not freely refined. With these parameters a coupling constant $J_{13}=-2.162 \mathrm{~cm}^{-1}$ was obtained.

At high temperatures $\chi_{\mathrm{M}} T$ converges against the expected value for four uncoupled manganese(II) atoms with a spin of $S=5 / 2$. At low temperatures two of these four atoms couple antiferromagnetically (Mn1 and Mn3). Hence $\chi_{\mathrm{M}} T$ converges against the expected value for two uncoupled manganese(II) atoms at low temperatures. The coupling constant $J_{13}=-2.162 \mathrm{~cm}^{-1}$ is quite small though. Thus the two manganese atoms are only weakly coupled.

\section{Electrochemistry}

In subsequent experiments the redox properties of $\mathbf{8}$ were examined by means of cyclic voltammetry. A glassy carbon and a platinum electrode were used as working and counter 
electrode. As reference a saturated calomel electrode was employed. The cyclic voltammograms of 8 were recorded in aqueous solution $\left(0.1 \mathrm{M} \mathrm{NaClO}_{4}\right)$. Figure 7.11 illustrates the recorded cyclic voltammogram. The inset depicts the respective square wave voltammogram.

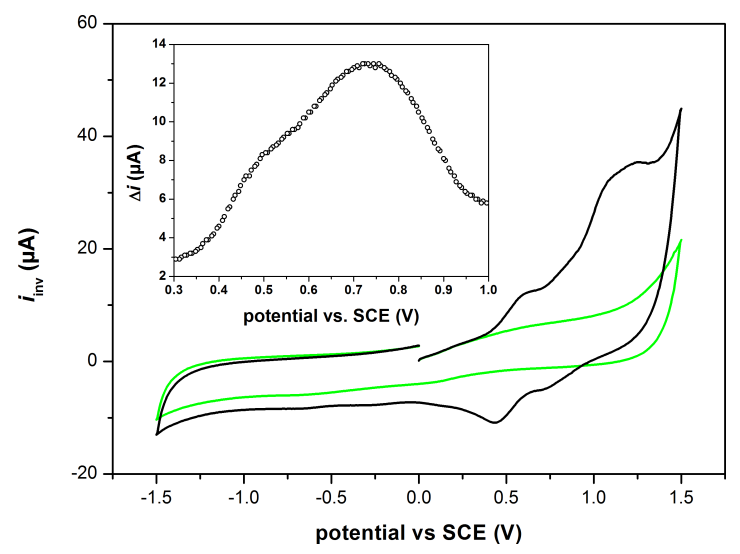

Figure 7.11: Cyclic voltammogram of 8 in neutral $\mathrm{H}_{2} \mathrm{O}\left(0.1 \mathrm{M} \mathrm{NaClO}_{4}\right)$ vs. SCE reference. The black line depicts the recorded voltammogram at $100 \mathrm{mV} / \mathrm{s}$. The green line marks the solvent background for comparison. The inset shows the corresponding square wave diagram.

The cyclic voltammogram of $\mathbf{8}$ displays only anodic oxidation events (black line). However, these events are far from ideally reversible. Possibly two pseudo reversible main oxidation events occur at $\sim 0.50 \mathrm{~V}$ and $0.73 \mathrm{~V}$ vs. SCE. Assuming from ESI mass spectra that 8 can be seen as dinuclear species in solution these oxidation events could be tentatively assigned in comparison to literature known dinuclear manganese complexes to $\mathrm{Mn}$ (II)Mn(III) and eventually $\mathrm{Mn}$ (III)Mn(IV) couples. 208 Above $1.23 \mathrm{~V}$ another irreversible oxidation event is observed, which could be attributed to another oxidation or an electrocatalytic event. In this range according to ÅKERMARK oxidations to Mn(IV) and $\mathrm{Mn}(\mathrm{v})$ occur. 208 As this oxidation event lies very close to the limit of the solvent window and the shape of this event is irreversible, it is not possible from the cyclic voltammogram to assign whether this oxidation event can be attributed to complex based oxidations or electrocatalysis. To further examine this behavior, the cyclic voltammogram usually is recorded at different scan rates. This was also done for $\mathbf{8}$, but did not lead to promising results. For a reversible redox couple the recorded current should be proportional to the square root of the scan rate. Ideal electrocatalytic events though are expected to be independent of the scan rate. 2051209 At different scan rates the positions of the recorded signals in the voltammogram of $\mathbf{8}$ changed significantly, which could be caused by structural or electronic rearrangements. Hence at this point from electrochemistry no valuable informations about the complex's ability to oxidize water can be extracted.

Two more attempts were made to elucidate the complex's electrochemistry and solution behavior. One experiment involved UV/Vis spectroelectrochemical measurements. Upon applying a potential of $1 \mathrm{~V}$ vs. SCE for $10 \mathrm{~min}$ no drastic changes of the UV/Vis spectrum of $\mathbf{8}$ were observed. Solely bands attributed to $\pi-\pi *$ transitions of the ligand shifted very slightly. In a second experiment another spectroscopy method was considered. As the complex's side arms contain carboxylate functions, which show prominent bands in IR 
spectroscopy, attempts were made to couple this method with electrochemistry. However, in IR spectroscopy the choice of solvents is limited. Solutions of $\mathbf{8}$ in methanol or water do not lead to sufficient spectral resolutions as these solvents absorb in the range of 1400 to $1600 \mathrm{~cm}^{-1}$, in which carboxylic stretches are usually observed.

\subsection{Summary}

In this chapter two new dinuclear iron complexes $\mathbf{6}$ and $\mathbf{7}$ and a tetranuclear manganese complex $\mathbf{8}$ were presented. All three complexes were structurally characterized and examined by means of cyclic voltammetry.

Complex 6 can mainly be regarded as a first diiron complex with the ligand $\mathbf{K}_{5} \mathbf{L} \mathbf{C O O}$ and a model to study the redox behavior of diiron complexes in a carboxylate-rich environment.

An advancement of $\mathbf{6}$ is its water soluble analogue, the diaqua diiron complex 7. Preliminary results show that the diiron(III) complex can be reduced and possibly be oxidized in neutral water, which is a key requirement for water oxidation catalysts. Solubility studies however revealed that a homogeneous catalyst system is best achieved at slightly basic $\mathrm{pH}$ values.

This implies that for future experiments new conditions for catalysis especially for these very versatile catalyst systems have to be developed. A starting point could be the choice of slightly basic, non coordinating buffer systems in aqueous solutions for electrocatalysis. Another approach for chemical water oxidation could involve the use of milder chemical oxidants.

The tetranuclear managanese complex 8 revealed very few parallels to the oxygen evolving complex in nature. Structurally the manganese atoms are bridged by the carboxylates of the ligand side arms which results in mono- and triatomic bridging modes between these sites. From mass spectrometry it is assumed that $\mathbf{8}$ nevertheless is a dinuclear manganese(II) species in solution. Redox potentials of $\mathbf{8}$ are comparable to literature known dinuclear manganese complexes in similar ligand environments. 208 However, the data obtained from scan rate dependent cyclic voltammogams of $\mathbf{8}$ were not consistent. These irregularities might result from different species in solution in the course of the examined electrochemical events and/or undesired side reactions. Insight into the species in solution could possibly be gained from measurements of the magnetic moment in solution in comparison with the recorded solid state data. 



\section{Summary and Conclusions}

In this work two new bis(pentadentate) pyrazolate based ligand scaffolds $\mathbf{H L}^{\mathbf{I m}}$ and $\mathbf{H L}^{\mathbf{P y}}$ were employed to emulate diiron sites from natural models. Their synthesis and characterization was documented in detail. With both ligand scaffolds new diiron(II) complexes (1a, 1b, 2a and $\mathbf{2 b}$ ) with nitrile coligands were prepared. All of these complexes were analytically and structurally characterized.

Optical spectroscopy, Mössbauer- and magnetic susceptibility measurements revealed an abrupt temperature dependent spin crossover phenomenon for $\mathbf{1 a}$ in solid state and a gradual SCO in nitrile solutions. A spin state switch from Fe(II)hs Fe(II)ls to two Fe(II)hs was observed. The spin crossover temperature $T_{1 / 2}$ was found to lie, dependent on the solvent, in the range between $248 \mathrm{~K}$ and $273 \mathrm{~K}$, which is in good agreement with documented $T_{1 / 2}$ values for dinuclear iron complexes. $148 / 149151$ Thermodynamic data independently derived from the gradual SCO in solution by SQUID magnetometry and UV/Vis spectroscopy were highly consistent. The low spin complexes $\mathbf{2 a}$ and $\mathbf{2} \mathbf{b}$ exhibited a high stability with pronounced optical signatures in the UV/Vis range.

All diiron(II) complexes readily reacted with molecular dioxygen at low temperatures. Reactions were mainly followed by means of UV/Vis and Mössbauer spectroscopy. For solutions of $\mathbf{1 a}$ and $\mathbf{1} \mathbf{b}$ a low temperature intermediate was trapped, precipitated and analyzed. Based on its spectral properties possible identities of this intermediate could be narrowed down to mainly two to three options, which involved the formation of a superoxo, hydroxo- or oxo-bridged diiron core. A final oxygenation product $\mathbf{3}$ from the oxidation of $\mathbf{1 a}$ in air was crystallographically characterized and revealed an asymmetrically coordinated diiron(III) hydroxo bridged core. Mössbauer parameters of $\mathbf{3}$ displayed a partial resemblance to the isolated intermediate and the high temperature oxygenation products, resulting from the reaction of $\mathbf{1 a}$ with dry dioxygen.

Oxygenations of $\mathbf{2 a}$ did not lead to very fruitful results. In general the activation of dioxygen in solution by $\mathbf{2 a}$ proceeded slower compared to $\mathbf{1 a}$ and $\mathbf{1 b}$ and at slightly higher temperatures. A putative intermediate was trapped on a single occasion by means of UV/Vis spectroscopy. A variety of decomposition products prevented further analysis.

The activation of nitric oxide by the diferrous complexes $\mathbf{1 a}$ and $\mathbf{2 a}$ proceeded cleanly to give new $\left[\{\mathrm{Fe}(\mathrm{NO})\}^{7}\right]_{2}$ diiron dinitrosyl complexes. These can be considered as mimics 
for the active sites of Flavodiiron proteins and they were structurally characterized. Their properties were elaborately studied by optical spectroscopy, Mössbauer and magnetic susceptibility measurements. While the spin state of the dinitrosyl complex 4 (prepared from 1a) was tentatively assigned to $3 / 2$ for both iron atoms, which agrees with literature known compounds, mixed spin states $(1 / 2$ and $3 / 2)$ were assigned for $\mathbf{5}$ (prepared from 2a). This unprecedented finding was supported by preliminary DFT-based geometry optimizations and the calculation of Mössbauer and IR data.

Intriguingly, despite their structural similarity the dinitrosyl complexes $\mathbf{4}$ and $\mathbf{5}$ followed different reaction pathways upon reduction. 4 was found to react to a diferrous complex $4^{\text {Red }}$ and a diiron tetranitrosyl $\left[\left\{\mathrm{Fe}(\mathrm{NO})_{2}\right\}^{9}\right]_{2}$ complex identified by optical spectroscopy. $4^{\text {Red }}$ was additionally isolated and structurally characterized. Interestingly, 5 did mimic FDP reactivity and reacted to a diferrous complex $5^{\text {Red }}$ under reductive conditions accompanied by the formation of $\mathrm{N}_{2} \mathrm{O}$. All reductions, (chemical and electrochemical) were followed by IR spectroscopy. $\mathrm{N}_{2} \mathrm{O}$ evolution was identified and quantified after reduction by headspace experiments. Hence $\mathbf{5}$ is aside from two previous examples of diiron dinitrosyl complexes in literature the only example with the ability to produce measurable amounts of $\mathrm{N}_{2} \mathrm{O}$ upon reduction. Other than in these systems the complex exhibits a mixed spin state.

In a short excursus the ability of $\mathbf{K}_{5} \mathbf{L}^{\mathbf{C O O}}$ to stabilize water soluble diiron complexes was demonstrated. A tetranuclear manganese complex was also isolated. These complexes might be suited potentially for water oxidation catalysis. In particular promising in this regard is the diaqua diiron complex $\mathbf{7}$ as it is able to coordinate two water molecules in a syn fashion and therefore in close proximity. Preliminary cyclic voltammetry experiments revealed that it might be experimentally possible to perform catalysis with the diaqua and the tetranuclear manganese complex. Experiments to improve the reaction conditions by changing the $\mathrm{pH}$ pointed towards a higher activity and solubility of the diaqua diiron complex in slightly basic media. For future works the development and use of non coordinating buffer systems can be a strategy to improve solubility. Also the choice of a different oxidant could be relevant for the success of catalysis in this respect.

As a perspective for future works it can be noted that the diiron(II) complexes of the ligand scaffolds $\mathbf{H L}^{\mathbf{I m}}$ and $\mathbf{H L}^{\mathbf{P y}}$ are now well understood. It is possible to activate both tested substrates, dioxygen as well as nitric oxide, with these multifunctional complexes. The oxygen chemistry of these complexes is still in its infancy due to challenging preparative conditions, e.g. working at very low temperatures and sensitivity of intermediates. Very promising on the other hand is the activation of nitric oxide, which is very worthwhile to follow up in future experiments. Structural evidence for the formation of a postulated tetranitrosyl diiron complex upon reduction is still needed.

Promising are also the preliminary works to perform water oxidation catalysis with the diaqua diiron complex $\mathbf{7}$. Diiron complexes with extended chelating ligands have not been employed in water oxidation catalysis although the trend in literature strives to use more abundant metals from the $3 \mathrm{~d}$ transition metal row. Hence this field of research is still not fully exploited and very promising. 


\section{Experimental}

\subsection{Procedures and Methods}

\section{Synthesis}

Chemicals for reactions were purchased from commonly known Suppliers (e.g. MERCK, Deutero, SigmaAldrich, Fluka, ABCR) and used without further purification. Chemicals that were not commercially available were either synthesized or already previously prepared within the group. Dry solvents were prepared by standard procedures, distilled and degassed either by bubbling argon through them or by freeze pump thaw degassing techniques prior to use. Air sensitive reactions were performed on a Schlenk line under a dry argon atmosphere or handled within a glovebox (MBRAUn Labmaster $s p$ ). Glassware used inside a glovebox or for Schlenk techniques was dried overnight. The iron salts $\mathrm{Fe}(\mathrm{OTf})_{2} \cdot 2 \mathrm{MeCN}$ and $\left[\mathrm{Fe}(\mathrm{MeCN})_{6}\right]\left(\mathrm{BF}_{4}\right)_{2}$ were prepared within the group according to literature procedures. $\frac{142 \mid 210}{}$ The side arm bis-((1-methyl-1H-imidazol-2-yl)methyl)amine $\left(\mathrm{sa}^{\mathrm{Im}}\right)$ was prepared according to a procedure reported by OBERHAUSEN et al.. 129 Microanalyses were performed by the "Analytical Laboratory" of the Institute of Inorganic Chemistry at the Georg-August-University Göttingen.

\section{Nitric Oxide Chemistry}

Nitrosylated compounds were prepared at a Schlenk line connected with an NO gas setup. $\mathrm{NO}_{(\mathrm{g})}$ was stored in a lecture bottle connected to a column packed with ascarite as absorbent. Via a three way valve selective evacuation and exposure to $\mathrm{NO}_{(\mathrm{g})}$ of tubes connecting the sample and NO lecture bottle was realized. Before purging the sample with gas, NO was passed through a cooling trap for gases in a dry ice/ethanol bath to filter off undesired contaminants and water.

\section{NMR Experiments}

Proton- $\left({ }^{1} \mathrm{H}-\mathrm{NMR}\right)$, carbon $\left({ }^{13} \mathrm{C}\left\{{ }^{1} \mathrm{H}\right\}-\mathrm{NMR}\right)$, correlated spectroscopy (H,H-COSY), heteronuclear single quantum coherence- (HSQC) and heteronuclear multiple bond correlation(HMBC) experiments were routinely performed on a BRUKER Avance 300 spectrometer at ambient temperature. Additional experiments were performed on a BRUKER Avance 500 spectrometer. Variable temperature NMR spectra were recorded on a BRUKER Avance 400 
spectrometer equipped with a cryostat and liquid nitrogen for cooling. Chemical shifts are reported in ppm relative to the solvent residual signal of the respective deuterated solvent. Multiplicities are abbreviated as following: $\mathrm{s}=$ singlet, $\mathrm{d}=$ doublet, $\mathrm{dd}=$ doublet of doublets and $t=$ triplet. Broadened signals are indexed with br. All coupling constants ${ }^{\mathrm{n}} J$ are given in $\mathrm{Hz}$.

\section{Mass Spectrometry}

Mass spectra via electron ionization (EI) were recorded on a FinNIGAN (Mat95) spectrometer. Electron spray ionization mass spectra (ESI-MS) were measured with an APPLIED BIOSYSTEMS (API 200) spectrometer, or air sensitive samples with a BRUKER HCT Ultra spectrometer connected to a MBRAUN glovebox. For air and moisture sensitive compounds exclusively dry and degassed solvents were used.

\section{Optical Spectroscopy}

\section{Infrared Spectrocopy}

Attenuated total reflectance spectra of solids and liquid films were recorded on a CARY 630 FTIR spectrometer (AGILENT) eqipped with with a DialPath and Diamond ATR accessory in a glovebox (MBRAun Unilab) under Argon atmosphere. Solids, that were not sensitive to air, (as KBr pellets) were recorded on a VERTEx 70 spectrometer. In the labs of Prof. Lehnert (University of Michigan) IR spectra , solid and liquid samples, were recorded on a PERKIN-Elmer BX spectrometer, which was constantly purged with a nitrogen stream. Air sensitive samples were transported from the nitrogen atmosphere of a glovebox in zip-lock bags to the spectrometer and were measured promptly.

\section{UV/Vis Spectroscopy}

UV/Vis spectra were either recorded on a VARIAN CARY 50 spectrometer equipped with a glassfiber optics cable and a Hellma quartz dip probe or on a CARY 60 spectrometer equipped with a cryostat (UNISOKU CoolSpek) cooled with liquid nitrogen. Air sensitive compounds were handled in Schlenk quartz cuvettes or, for the dip probe, in custom made Schlenk vessels. For titrations or addition of substance during a measurement cuvettes with quick fit locks and customized septa were used. In the labs of Prof. Lehnert (University of Michigan) UV/Vis spectra were recorded on a Analytical Jena Specord 600 instrument either with a quartz dip probe inside a MBRAUN glovebox (nitrogen atmosphere) or in quartz cuvettes with quick fit locks.

\section{Cyclic Voltammetry and Electrochemistry}

Cyclic voltammograms were recorded with a EG\&G 272 A potentiostat or, in the group of Prof. Lehnert (University of Michigan), with a $\mathrm{CH}$ instruments CHI600E workstation. A glassy carbon electrode was implemented as working electrode and a platinum electrode as counter electrode. Reference electrode and conducting salt for the respective experiments are mentioned separately in line with the evaluation of the experiment. Air sensitive experiments were conducted in custom made Schlenk cells either in or outside of a glovebox. 
Conducting salt solutions were generally degassed prior to the experiment. IR-Echem experiments were conducted in IR solution cells with $\mathrm{CaF}_{2}$ windows equipped with two platinum meshs as working and counter electrode $(8 \times 10 \mathrm{~mm}$ Pt mesh $(100 \mathrm{mesh}, 99.9 \%$, Aldrich) for the working electrode, $3 \times 10 \mathrm{~mm}$ Pt mesh for the counter electrode) and a silver wire $((0.1 \mathrm{~mm}, 99.9 \%)$ as reference. Spectra were recorded either on a PERKIN-Elmer BX spectrometer with mobile CHI600E workstation or on a VERTEX 70 spectrometer with a CHI-660 (IJ-Cambria scientific) potentiostat.

\section{Crystallography}

X-ray data were collected on a STOE IPDS II diffractometer with an area detector (graphite monochromated Mo-K $\alpha$ radiation, $\lambda=0.71073 \AA$ ) by use of $\omega$ scans at at $133 \mathrm{~K}$. The structures were solved by direct methods SHELXS-14 211 and refined on $\mathrm{F}^{2}$ with all reflections using SHELXL-14. 211] Non-hydrogen atoms were refined anisotropically unless noted otherwise. Hydrogen atoms were placed in calculated positions and assigned to an isotropic displacement parameter of 1.2 / $1.5 \mathrm{Ueq}(\mathrm{C})$. Face-indexed absorption corrections were performed by the program X-RED. 212

\section{Mössbauer Spectroscopy}

Mössbauer spectra were recorded on a WissEl spectrometer with constant alternating acceleration and a ${ }^{57} \mathrm{Co}$ source homogeneously diffused in a $\mathrm{Rh}$ matrix. The spectrometer was built of a MR 360 Drive unit, a MVT-1000 velocity transducer and a LND 45431 proportional counter mounted on a LINOS precision bench. Source, absorber and detector were arranged in linear geometry. For low temperature measurements (below $80 \mathrm{~K}$ ) a Janis SHI closed-cycle helium cryostat was used. As detector a 512 channel analyzer was used. isomer shifts $\delta$ are given relative to iron metal at ambient temperature. The acquired data were fit with the Mfit program (E. Bill, Max-Planck Institute for Chemical Energy Conversion, Mülheim/Ruhr, Germany). 213]

Solid crystalline or polycrystalline samples were placed in sealable PEEK tablet and covered with mineral oil (typically motor oil) if a solvent loss was apparent. The tablet was mounted in a sample holder and was flashfrozen in liquid nitrogen. For solution samples at least $20 \mathrm{mg}$ of crystalline or powder sample was dissolved in $1 \mathrm{~mL}$ of the respective solvent. The solution was placed in a PEEK cup which was sealed and immediately flashfrozen. For air sensitive samples these procedures were performed inside a glovebox. Data were collected for 1-2 d depending on the amount and quality of sample until a sufficient signal-to-noise ratio was reached.

\section{SQUID Magnetometry}

Temperature dependent magnetic susceptibility measurements were conducted with a Quantum-Design MPMS XL-5 SQUID magnetometer equipped with a 5 T magnet. Measurements were recorded in the range from 295 to $2 \mathrm{~K}$. Solid polycrystalline samples were placed in a gelatin capsule inside a glovebox. To avoid orientation of the crystalline material, the samples were fixed with a drop of a low viscosity perfluoropolyether based inert 
oil Fomblin Y45. The capsule was closed with a lid and placed in a diamagnetic sample holder (PTFE straw).

Raw data files for the magnetic moment were corrected for the diamagnetic contribution of the gelatine capsule according to $M_{\text {dia }}$ (capsule) $=\chi_{\mathrm{g}} \times m \times H$. The gram susceptibility of the gelatine capsule was experimentally obtained. The molar susceptibility data were corrected for the diamagnetic contribution using Pascal constants and the increment method according to Haberditzl. 214]215.

Magnetic properties were simulated with the julX program [174] (E. Bill, Max-Planck Institute for Chemical Energy Conversion, Mülheim/Ruhr, Germany, 2008) using a HeisenbergDirac-van-Vleck Hamiltonian (HDvV) including isotropic exchange interaction, zerofield and Zeeman splitting.

$$
\begin{gathered}
\hat{H}=\hat{H}^{\mathrm{exc}}+\hat{H}^{\mathrm{ZFS}}+\hat{H}^{\mathrm{Zeem}} \\
\hat{H}^{\mathrm{exc}}=-2 \sum_{i>j} J_{i j} \hat{S}_{i} \hat{S}_{j} \\
\hat{H}^{\mathrm{ZFS}}=\sum_{i} D_{i}\left(\hat{S}_{z, i}^{2}-\frac{1}{3} S_{i}\left(S_{i}+1\right) \frac{E_{i}}{D_{i}}\left(\hat{S}_{x, i}^{2}-\hat{S}_{y, i}^{2}\right)\right) \\
\hat{H}^{\mathrm{Zeem}}=\sum_{i} \mu_{\mathrm{B}}\left(\hat{S}_{x, i} g_{x, i} B_{x}+\hat{S}_{y, i} g_{y, i} B_{y}+\hat{S}_{z, i} g_{z, i} B_{z}\right)
\end{gathered}
$$

Temperature independent paramagnetism and paramagnetic impurities were included according to $\chi_{\text {calc }}=(1-P I) \cdot \chi+P I \cdot \chi_{\text {mono }}+T I P$.

Measurements of liquid samples were conducted in melt off glass tubes. The aquired data were corrected for contributions of the respective solvent and the glass tube.

\section{DFT Calculations}

Single point calculations on the coordinates of the cation of the diiron dinitrosyl complexes obtained by x-ray diffraction experiments were performed with the ORCA program (Version 3.0.3). 216] The optimization of the hydrogen atom positions and broken-symmetry calculations were performed by unrestricted dft methods, using the BP86 hybrid functional with def2-tzvp and def2-tzvp/j basis sets. 217|218. For IR data the numfreq option was used. Mössbauer parameters were calculated at the DFT level of theory (unrestricted DFT calculations with the B3LYP functional, def2-tzvp basis set + enlarged CP(PPP) basis set for the Fe atoms). The isomer shifts were obtained using the correlation formula and parameters reported by Neese et al. 168 


\subsection{Synthesis of the Pyrazole Building Block}

\section{Dimethylpyridin-2,6-dicarboxylate (pz2)}

Pyridine-2,6-dicarboxylic acid (100 g, $598 \mathrm{mmol}, 1.00$ eq.) was suspended in dry methanol $(420 \mathrm{~mL})$. To this suspension conc. sulfuric acid $(30 \mathrm{~mL})$ was added dropwise over the course of $1 \mathrm{~h}$. The reaction mixture heated to reflux for $1 \mathrm{~d}$. After cooling, ice water $(330 \mathrm{~g})$ was added and the product was filtered off. The filtrate was neutralized with $\mathrm{Na}_{2} \mathrm{CO}_{3}$, filtered and extracted with dichloromethane $(3 \times 100 \mathrm{~mL})$. Removal of the solvent yielded additional product as a white solid (112 mg, $229 \mathrm{mmol}, 77 \%$ ).

${ }^{1} \mathbf{H}-\mathbf{N M R}\left(300 \mathrm{MHz}, 298 \mathrm{~K}, \mathrm{CDCl}_{3}\right): \delta(\mathrm{ppm})=8.32\left(\mathrm{~d},{ }^{3} J_{\mathrm{H}, \mathrm{H}}=7.8 \mathrm{~Hz}, 2 \mathrm{H}, 3-\mathrm{H}, 5-\mathrm{H}\right)$, $8.03\left(\mathrm{t},{ }^{3} J_{\mathrm{H}, \mathrm{H}}=7.8 \mathrm{~Hz}, 1 \mathrm{H}, 4-\mathrm{H}\right), 4.03\left(\mathrm{~s}, 6 \mathrm{H}, \mathrm{CH}_{3}\right)$.

${ }^{13} \mathbf{C}\left\{{ }^{1} \mathbf{H}\right\}$-NMR $\left(75 \mathrm{MHz}, 298 \mathrm{~K}, \mathrm{CDCl}_{3}\right): \delta(\mathrm{ppm})=165(\mathrm{CO}), 148(\mathrm{C} 2, \mathrm{C} 6), 138(\mathrm{C} 4)$, $128(\mathrm{C} 3, \mathrm{C} 5), 53\left(\mathrm{CH}_{3}\right)$.

MS (EI): m/z $(\%)=137(100)\left[\mathrm{Py}+\mathrm{COOCH}_{3}\right]^{+}, 105(40)[\mathrm{Py}+\mathrm{CO}]^{+}$.

\section{Methyl-6-(hydroxymethyl)picolinate (pz3)}

To a suspension of dimethylpyridine-2,6-dicarboxylate $(20.0 \mathrm{~g}, 41.0 \mathrm{mmol}, 2.00 \mathrm{eq}$.$) in dry$ methanol $(200 \mathrm{~mL}) \mathrm{NaBH}_{4}(2.33 \mathrm{~g}, 61.6 \mathrm{mmol}, 3.00$ eq. $)$ was added in small portions. The reaction mixture was refluxed for $1 \mathrm{~d}$. After cooling, the volume of the solvent was reduced by half under reduced pressure and water $(50 \mathrm{~mL})$ was added. The volume of the solution was further reduced to final $50 \mathrm{~mL}$. The residue was extracted with dichloromethane $(4 \times 20 \mathrm{~mL})$. After removal of the solvent a white solid was obtained. For further purification the product was either recrystallized from methanol or purified by column chromatography (dichloromethane/methanol, 40:1). Yield (10.0 g, 50.8 mmol, $73 \%)$.

${ }^{1} \mathbf{H}-\mathbf{N M R}\left(300 \mathrm{MHz}, 298 \mathrm{~K}, \mathrm{CDCl}_{3}\right): \delta(\mathrm{ppm})=8.04\left(\mathrm{~d},{ }^{3} J_{\mathrm{H}, \mathrm{H}}=7.8 \mathrm{~Hz}, 1 \mathrm{H}, 3-\mathrm{H}\right), 7.85$ $\left(\mathrm{t},{ }^{3} J_{\mathrm{H}, \mathrm{H}}=7.8 \mathrm{~Hz}, 1 \mathrm{H}, 4-\mathrm{H}\right), 7.53\left(\mathrm{~d},{ }^{3} J_{\mathrm{H}, \mathrm{H}}=7.8 \mathrm{~Hz}, 1 \mathrm{H}, 5-\mathrm{H}\right), 4.87\left(\mathrm{~s}, 2 \mathrm{H}, \mathrm{CH}_{2}\right), 4.00$ $\left(\mathrm{s}, 3 \mathrm{H}, \mathrm{CH}_{3}\right)$.

${ }^{13} \mathbf{C}\left\{{ }^{1} \mathbf{H}\right\}$-NMR $\left(75 \mathrm{MHz}, 298 \mathrm{~K}, \mathrm{CDCl}_{3}\right): \delta(\mathrm{ppm})=166(\mathrm{CO}), 161(\mathrm{C} 6), 147(\mathrm{C} 2)$, $138(\mathrm{C} 4), 124(\mathrm{C} 3, \mathrm{C} 5), 65\left(\mathrm{CH}_{2}\right), 53\left(\mathrm{CH}_{3}\right)$.

MS (EI): m/z (\%) = $166(60)[\mathrm{M}]^{+}, 109(100)\left[\mathrm{M}+\mathrm{COOCH}_{3}\right]^{+}$. 


\section{(6-(Hydroxymethyl)pyridin-2-yl)ethanon (pz4)}

To a suspension of freshly prepared NaOEt $(6.18 \mathrm{~g}, 90.2 \mathrm{mmol}, 1.51$ eq. $)$ in dry toluene, freshly distilled ethyl acetate $(37.5 \mathrm{~mL}, 380 \mathrm{mmol}, 6.35$ eq.) was added. The suspension was stirred for $30 \mathrm{~min}$ at ambient temperature followed by the addition of methyl6-(hydroxymethyl)picolinate $(10.0 \mathrm{~g}, 59.8 \mathrm{mmol}, 1.00 \mathrm{eq}$.) and additional dry toluene $(150 \mathrm{~mL})$. The reaction mixture was heated to reflux for $6 \mathrm{~h}$ and stirred another $10 \mathrm{~h}$ at ambient temperature. The solvent was removed under reduced pressure and sulfuric acid (20 w\%, $75 \mathrm{~mL}$ ) was added to the residue. This suspension was heated to reflux another $6 \mathrm{~h}$. After the $\mathrm{CO}_{2}$ evolution had seized the reaction mixture was cooled to $0{ }^{\circ} \mathrm{C}$ and the $\mathrm{pH}$ of the mixture was increased to 8 by the addition of $\mathrm{Na}_{2} \mathrm{CO}_{3}$ and $\mathrm{NaOH}$. The reaction mixture was filtered and the residue was washed with water $(2 \times 25 \mathrm{~mL})$. The filtrate was extracted with dichloromethane $(6 \times 50 \mathrm{~mL})$ and the combined organic fractions were dried over $\mathrm{Na}_{2} \mathrm{SO}_{4}$. Evaporation of the solvent yielded the ketone as dark red oil ( $4.01 \mathrm{~g}$, $26.5 \mathrm{mmol}, 44 \%$ ).

${ }^{1} \mathbf{H}-\mathbf{N M R}\left(300 \mathrm{MHz}, 298 \mathrm{~K}, \mathrm{CDCl}_{3}\right): \delta(\mathrm{ppm})=7.97\left(\mathrm{~d},{ }^{3} J_{\mathrm{H}, \mathrm{H}}=7.6 \mathrm{~Hz}, 1 \mathrm{H}, 3-\mathrm{H}\right), 7.85$ $\left(\mathrm{t},{ }^{3} J_{\mathrm{H}, \mathrm{H}}=7.6 \mathrm{~Hz}, 1 \mathrm{H}, 4-\mathrm{H}\right), 7.43\left(\mathrm{~d},{ }^{3} J_{\mathrm{H}, \mathrm{H}}=7.6 \mathrm{~Hz}, 1 \mathrm{H}, 5-\mathrm{H}\right), 4.84\left(\mathrm{~s}, 2 \mathrm{H}, \mathrm{CH}_{2}\right), 2.74$ (s, $\left.2 \mathrm{H}, \mathrm{CH}_{3}\right)$.

${ }^{13} \mathbf{C}\left\{{ }^{1} \mathbf{H}\right\}$-NMR $\left(75 \mathrm{MHz}, 298 \mathrm{~K}, \mathrm{CDCl}_{3}\right): \delta(\mathrm{ppm})=200(\mathrm{CO}), 159(\mathrm{C} 6), 152(\mathrm{C} 2), 138$ (C5), $124(\mathrm{C} 4), 1201(\mathrm{C} 3), 64\left(\mathrm{CH}_{2} \mathrm{OH}\right), 26\left(\mathrm{CH}_{3}\right)$.

\section{3,5-Bis(6-hydroxymethyl-2-pyridyl)-1H-pyrazole (pz6)}

A suspension of freshly prepared NaOEt $(4.34 \mathrm{~g}, 63.9 \mathrm{mmol}, 1.12$ eq.) in dry toluene $(90 \mathrm{~mL})$ and methyl-6-(hydroxymethyl)picolinate $(9.51 \mathrm{~g}, 56.9 \mathrm{mmol}, 1.00 \mathrm{eq}$.$) \quad was$ warmed to $50^{\circ} \mathrm{C}$. To this suspension a solution of 1-(6-(hydroxymethyl)pyridin-2yl)ethanon $(8.95 \mathrm{~g}, 59.2 \mathrm{mmol}, 1.04$ eq. $)$ in dry toluene $(110 \mathrm{~mL})$ was added dropwise over the course of $6 \mathrm{~h}$. The reaction mixture was stirred another $3 \mathrm{~h}$ at $50{ }^{\circ} \mathrm{C}$ and at ambient temperature overnight. After that the red precipitate was filtered. In an ice bath the residue was suspended in a solution of acetic acid $(3.80 \mathrm{~mL})$ and water $(44 \mathrm{~mL})$. Filtration and drying of the orange precipitate yielded the diketone, which was directly dissolved in ethanol $(90 \mathrm{~mL})$. To this suspension hydrazine-hydrate $(64 \mathrm{wt} \%, 11 \mathrm{~mL}, 141 \mathrm{mmol}$ hydrazine, 2.48 eq.) was added and the reaction mixture was heated to reflux at $105^{\circ} \mathrm{C}$ for $4 \mathrm{~h}$. After cooling the mixture was stirred another $12 \mathrm{~h}$ at ambient temperature. The solvent and excess hydrazine was removed in vacuo. The residue was suspended in water $(15 \mathrm{~mL})$ in an ultrasonic bath and layered with diethyl ether. The slight yellow precipitate was filtered and dried. Yield (5.86 g, $20.8 \mathrm{mmol}, 37 \%$ ).

${ }^{1} \mathbf{H}-\mathbf{N M R}\left(300 \mathrm{MHz}, 298 \mathrm{~K}, \mathrm{DMSO}_{-} \mathrm{d}_{6}\right): \delta(\mathrm{ppm})=13.57\left(\mathrm{~s}_{\mathrm{br}}, 1 \mathrm{H}, \mathrm{NH}\right), 7.79-7.86(\mathrm{~m}$, $\left.4 \mathrm{H}, 3-\mathrm{H}^{\mathrm{Py}}, 5-\mathrm{H}^{\mathrm{Py}}\right), 7.37-7.39\left(\mathrm{~m}, 3 \mathrm{H}, 4-\mathrm{H}^{\mathrm{Py}}, \mathrm{H}^{\mathrm{Pz}}\right), 5.42\left(\mathrm{~s}_{\mathrm{br}}, 2 \mathrm{H}, \mathrm{OH}\right), 4.61\left(\mathrm{~s}, 4 \mathrm{H}, \mathrm{CH}_{2}\right)$.

${ }^{13} \mathbf{C}\left\{{ }^{1} \mathbf{H}\right\}$-NMR $\left(75 \mathrm{MHz}, 298 \mathrm{~K}, \mathrm{DMSO}_{\mathrm{d}}\right): \delta(\mathrm{ppm})=162\left(\mathrm{C} 6^{\mathrm{Py}}\right), 138\left(\mathrm{C}^{\mathrm{Py}} / \mathrm{C} 5^{\mathrm{Py}}\right)$, 
$119\left(\mathrm{C} 4^{\mathrm{Py}}\right), 118\left(\mathrm{C}^{\mathrm{Py}} / \mathrm{C} 5^{\mathrm{Py}}\right), 102\left(\mathrm{C} 4^{\mathrm{Pz}}\right), 64\left(\mathrm{CH}_{2}\right)\left(\mathrm{C} 2^{\mathrm{Py}}\right.$ and $\mathrm{C} 3^{\mathrm{Pz}} / \mathrm{C} 5^{\mathrm{Pz}}$ not observed $)$.

MS (ESI $\left.{ }^{+}, \mathbf{M e O H}\right): \mathrm{m} / \mathrm{z}(\%)=587(50)[2 \mathrm{M}+\mathrm{Na}]^{+}, 305(87)[\mathrm{M}+\mathrm{Na}]^{+}, 283(100)$ $[\mathrm{M}+\mathrm{H}]^{+}$.

\section{3,5-Bis(6-chlormethyl-2-pyridyl)-1H-pyrazole (pz7)}

3,5-bis(6-hydroxymethyl-2-pyridyl)- $1 H$-pyrazole $(1.00 \mathrm{~g}, 2.54 \mathrm{mmol}, 1.00 \mathrm{eq}$.$) \quad was sus-$ pended in freshly distilled thionyl chloride $(10 \mathrm{~mL})$ and stirred for $3 \mathrm{~d}$ at ambient temperature. Excess thionyl chloride was distilled off and the residue suspended in a mixture of $\mathrm{NaHCO}_{3}(3.30 \mathrm{~g}, 39.3 \mathrm{mmol}, 15.5$ eq. $)$ in water $(10 \mathrm{~mL})$ and ethyl acetate $(25 \mathrm{~mL})$. The suspension was filtered and the residue washed with ethyl acetate. The layers of the filtrate were separated. The water layer was extracted with ethyl acetate $(3 \times 15 \mathrm{~mL})$. The combined organic layers were dried over $\mathrm{Na}_{2} \mathrm{SO}_{4}$ and the solvent was removed. The product was further purified by filtration over a silica column (ethyl acetate) to yield a yellow solid (585 mg, $1.83 \mathrm{mmol}, 72 \%$ ).

${ }^{1} \mathbf{H}-\mathbf{N M R}\left(300 \mathrm{MHz}, 298 \mathrm{~K}, \mathrm{DMSO}_{-} \mathrm{d}_{6}\right): \delta(\mathrm{ppm})=13.77(\mathrm{~s}, 1 \mathrm{H}, \mathrm{NH}), 7.82-7.90(\mathrm{~m}$, $\left.4 \mathrm{H}, 3-\mathrm{H}^{\mathrm{Py}}, 5-\mathrm{H}^{\mathrm{Py}}\right), 7.42\left(2 \times \mathrm{d},{ }^{3} J_{\mathrm{H}, \mathrm{H}}=6.9 \mathrm{~Hz}, 2 \mathrm{H}, 4-\mathrm{H}^{\mathrm{Py}}\right), 7.37\left(\mathrm{~s}, 1 \mathrm{H}, \mathrm{H}^{\mathrm{Pz}}\right), 4.75(\mathrm{~s}$, $\left.4 \mathrm{H}, \mathrm{CH}_{2}\right)$.

${ }^{13} \mathbf{C}\left\{{ }^{1} \mathbf{H}\right\}$-NMR $\left(75 \mathrm{MHz}, 298 \mathrm{~K}, \mathrm{DMSO}-\mathrm{d}_{6}\right): \delta(\mathrm{ppm})=156\left(\mathrm{C6}^{\mathrm{Py}}\right), 138\left(\mathrm{C}^{\mathrm{Py}} / \mathrm{C} 5^{\mathrm{Py}}\right)$, $122\left(\mathrm{C}^{\mathrm{Py}}\right), 119\left(\mathrm{C}^{\mathrm{Py}} / \mathrm{C} 5^{\mathrm{Py}}\right), 103\left(\mathrm{C}^{\mathrm{Pz}}\right), 47\left(\mathrm{CH}_{2}\right),\left(\mathrm{C}^{\mathrm{Py}}\right.$ and $\mathrm{C} 3^{\mathrm{Pz}} / \mathrm{C} 5^{\mathrm{Pz}}$ not observed $)$.

MS (EI): $\mathrm{m} / \mathrm{z}(\%)=320(63)\left[\mathrm{M}\left({ }^{37} \mathrm{Cl}\right)\right]^{+}, 318(100)\left[\mathrm{M}\left({ }^{35} \mathrm{Cl}\right)\right]^{+}, 283(15)[\mathrm{M}-\mathrm{Cl}]^{+}$.

\section{3,5-Bis(6-brommethyl-2-pyridyl)-1H-pyrazole (pz8)}

3,5-bis(6-hydroxymethyl-2-pyridyl)- $1 H$-pyrazole $(1.65 \mathrm{~g}, 5.84 \mathrm{mmol}, 1.00 \mathrm{eq}$.$) \quad was sus-$ pended in a mixture of hydrogen bromide ( $48 \%$ in aqueous solution, $20 \mathrm{~mL}$ ) and sulfuric acid (conc., $3.4 \mathrm{~mL}$ ) and heated to reflux for $1 \mathrm{~d}$. After another addition of hydrogen bromide $(5 \mathrm{~mL})$ and sulfuric acid $(1.5 \mathrm{~mL})$ the reaction mixture was refluxed another $12 \mathrm{~h}$. The reaction mixture was cooled to $0{ }^{\circ} \mathrm{C}$ and water $(23 \mathrm{~mL})$ was added. By the addition of $\mathrm{Na}_{2} \mathrm{CO}_{3}$ a $\mathrm{pH}$ of 8 was obtained. The reaction mixture was filtered and the crude product was purified by column chromatography (silica, ethyl acetate) to obtain a white solid (734 mg, $1.80 \mathrm{mmol}, 31 \%$ ).

${ }^{1}$ H-NMR $\left(300 \mathrm{MHz}, 298 \mathrm{~K}, \mathrm{DMSO}-\mathrm{d}_{6}\right): \delta(\mathrm{ppm})=13.76\left(\mathrm{~s}_{\mathrm{br}}, 1 \mathrm{H}, \mathrm{NH}\right), 7.87-7.94(\mathrm{~m}$, $\left.4 \mathrm{H}, 3-\mathrm{H}^{\mathrm{Py}}, 5-\mathrm{H}^{\mathrm{Py}}\right)$, 7.44-7.52 (m, 3 H, 4- $\left.\mathrm{H}^{\mathrm{Py}}, \mathrm{H}^{\mathrm{Pz}}\right), 4.74\left(\mathrm{~s}, 4 \mathrm{H}, \mathrm{CH}_{2}\right)$.

${ }^{13} \mathbf{C}\left\{{ }^{1} \mathbf{H}\right\}$-NMR $\left(75 \mathrm{MHz}, 298 \mathrm{~K}, \mathrm{DMSO}-\mathrm{d}_{6}\right): \delta(\mathrm{ppm})=160\left(\mathrm{C} 6^{\mathrm{Py}}\right), 146\left(\mathrm{C}^{\mathrm{Pz}} / \mathrm{C} 5^{\mathrm{Pz}}\right)$, $145\left(\mathrm{C}^{\mathrm{Py}}\right), 141\left(\mathrm{C}^{\mathrm{Py}} / \mathrm{C}^{\mathrm{Py}}\right), 121\left(\mathrm{C}^{\mathrm{Py}}\right), 120\left(\mathrm{C}^{\mathrm{Py}} / \mathrm{C} 5^{\mathrm{Py}}\right), 104\left(\mathrm{C}^{\mathrm{Pz}}\right), 62\left(\mathrm{CH}_{2}\right)$. 
MS (EI): m/z (\%) = $408(100)[\mathrm{M}]^{+}, 329(56)[\mathrm{M}-\mathrm{Br}]^{+}, 248(70)[\mathrm{M}-2 \mathrm{Br}]^{+}$.

\subsection{Synthesis of the Ligands}

\section{Synthesis of $\mathrm{HL}^{\mathrm{Im}}$}

A suspension of 3,5-bis(6-chlormethyl-2-pyridyl)- $1 H$-pyrazole ( $1.70 \mathrm{~g}, 5.33 \mathrm{mmol}, 1.00 \mathrm{eq}$.), bis((1-methyl-1H-imidazol-2-yl)methyl)amine (2.18 g, $10.6 \mathrm{mmol}, 2.00$ eq. $)$ and excess of $\mathrm{Na}_{2} \mathrm{CO}_{3}$ in $\mathrm{MeCN}$ was heated to reflux for $5 \mathrm{~d}$. The suspension was filtered. The solvent of the filtrate was removed and the residue dissolved in dichloromethane. This suspension was filtered and the solvent of the filtrate removed in vacuo. The residue was washed with demineralized water and redissolved in dichloromethane. Filtration and removal of the solvent yielded $\mathrm{HL}^{\mathrm{Im}}$ (433 $\left.\mathrm{mg}, 0.66 \mathrm{mmol}, 12 \%\right)$.

${ }^{1} \mathbf{H}-\mathbf{N M R}\left(500 \mathrm{MHz}, 298 \mathrm{~K}, \mathrm{MeOH}-\mathrm{d}_{4}\right) \delta(\mathrm{ppm})=7.79\left(\mathrm{dd},{ }^{3} J_{\mathrm{H}, \mathrm{H}}=8.0 \mathrm{~Hz}, 4 \mathrm{H}, 3-\mathrm{H}^{\mathrm{Py}}\right.$, $\left.5-\mathrm{H}^{\mathrm{Py}}\right), 7.42\left(\mathrm{~s}, 1 \mathrm{H}, \mathrm{H}^{\mathrm{Pz}}\right), 7.12\left(\mathrm{~d},{ }^{3} J_{\mathrm{H}, \mathrm{H}}=8.0 \mathrm{~Hz}, 2 \mathrm{H}, 4-\mathrm{H}^{\mathrm{Py}}\right), 6.99\left(\mathrm{~d},{ }^{3} J_{\mathrm{H}, \mathrm{H}}=1.3 \mathrm{~Hz}\right.$, $\left.4 \mathrm{H}, \mathrm{H}^{\mathrm{Im}}\right), 6.86\left(\mathrm{~d},{ }^{3} J_{\mathrm{H}, \mathrm{H}}=1.3 \mathrm{~Hz}, 4 \mathrm{H}, \mathrm{H}^{\mathrm{Im}}\right), 3.84\left(\mathrm{~s}, 8 \mathrm{H}, \mathrm{CH}_{2}\right), 3.82\left(\mathrm{~s}, 4 \mathrm{H}, \mathrm{CH}_{2}\right), 3.43$ (s, $\left.12 \mathrm{H}, \mathrm{CH}_{3}\right)$.

${ }^{13} \mathbf{C}\left\{{ }^{1} \mathbf{H}\right\}-\mathbf{N M R}\left(126 \mathrm{MHz}, 298 \mathrm{~K}, \mathrm{MeOH}-\mathrm{d}_{4}\right) \delta(\mathrm{ppm})=159.6\left(\mathrm{C}^{\mathrm{Py}}, \mathrm{C} 2^{\mathrm{Py}}\right), 146.4$ $\left(\mathrm{C}_{\mathrm{q}}^{\mathrm{Im}}\right), 138.7\left(\mathrm{C} 3 / 5^{\mathrm{Py}}\right), 127.1\left(\mathrm{CH}^{\mathrm{Im}}\right), 124.0\left(\mathrm{C}^{\mathrm{Py}}\right), 123.5\left(\mathrm{CH}^{\mathrm{Im}}\right), 119.7\left(\mathrm{C} 3 / 5^{\mathrm{Py}}\right), 103.2$ $\left(\mathrm{C} 4{ }^{\mathrm{Pz}}\right), 60.6\left(\mathrm{CH}_{2}\right), 49.5\left(\mathrm{CH}_{2}\right), 33.0\left(\mathrm{CH}_{3}\right)\left(\mathrm{C} 3^{\mathrm{Pz}} / \mathrm{C} 5^{\mathrm{Pz}}\right.$ not observed$)$.

MS $\left(\mathbf{E S I}^{+}, \mathbf{M e O H}\right): \mathrm{m} / \mathrm{z}(\%)=679.4(100)[\mathrm{M}+\mathrm{Na}]^{+}, 351.2(15)[\mathrm{M}+\mathrm{Na}]^{2+}$.

IR(ATR, solid) $1729(\mathrm{~m}), 1678(\mathrm{w}), 1592(\mathrm{~s}), 1570(\mathrm{~s}), 1530(\mathrm{w}), 1497(\mathrm{w}), 1442(\mathrm{~m})$, $1414(\mathrm{~m}), 1368(\mathrm{w}), 1280(\mathrm{~s}), 1242(\mathrm{~m}), 1141(\mathrm{~m}), 1080(\mathrm{~m}), 1041(\mathrm{~s}), 989(\mathrm{~s}), 921(\mathrm{~s})$, $844(\mathrm{~m}), 793(\mathrm{~s}), 736(\mathrm{~s}), 658(\mathrm{~s})$.

\section{Synthesis of $\mathrm{HL}^{\mathrm{Py}}$}

3,5-bis(6-brommethyl-2-pyridyl)- $1 H$-pyrazole (1.03 g, $2.52 \mathrm{mmol}, 1.00$ eq.) was suspended in a 1:1 mixture of methanol and tetrahydrofuran. Subsequently dipicolylamine $(1.00 \mathrm{~g}$, $5.05 \mathrm{mmol}, 2.00$ eq.) as well as approximately 10.0 eq. of $\mathrm{Na}_{2} \mathrm{CO}_{3}$ were added. The reaction mixture was heated to reflux for $24 \mathrm{~h}$. After cooling to ambient temperature the mixture was filtered. The solvent of the filtrate was removed and the residue redissolved in dichloromethane. Again the solution was filtered and the solvent of the filtrate removed. The residue was dried in vacuo to afford $\mathrm{HL}^{\mathrm{Py}} \cdot \mathrm{HBr}(1.32 \mathrm{~g}, 1.81 \mathrm{mmol}, 72 \%)$.

${ }^{1} \mathbf{H}-\mathbf{N M R}\left(400 \mathrm{MHz}, 298 \mathrm{~K}, \mathrm{MeCN}_{-} \mathrm{d}_{3}\right): \delta(\mathrm{ppm})=8.51\left(\mathrm{ddd},{ }^{3,4} J_{\mathrm{H}, \mathrm{H}}=4.9,1.7,0.9 \mathrm{~Hz}\right.$, $\left.4 \mathrm{H}, 10-\mathrm{H}^{\mathrm{Py}}\right), 7.75-7.80\left(\mathrm{~m}, 4 \mathrm{H}, 3 / 5-\mathrm{H}^{\mathrm{Py}}\right), 7.72\left(\mathrm{td},{ }^{3,4} J_{\mathrm{H}, \mathrm{H}}=7.6,1.7 \mathrm{~Hz}, 4 \mathrm{H}, 9-\mathrm{H}^{\mathrm{Py}}\right)$, $7.63\left(\mathrm{~d},{ }^{3} J_{\mathrm{H}, \mathrm{H}}=7.6 \mathrm{~Hz}, 4 \mathrm{H}, 8-\mathrm{H}^{\mathrm{Py}}\right), 7.40-7.44\left(\mathrm{~m}, 2 \mathrm{H}, 4-\mathrm{H}^{\mathrm{Py}}\right), 7.35\left(\mathrm{~s}, 1 \mathrm{H}, \mathrm{H}^{\mathrm{Pz}}\right), 7.20$ 


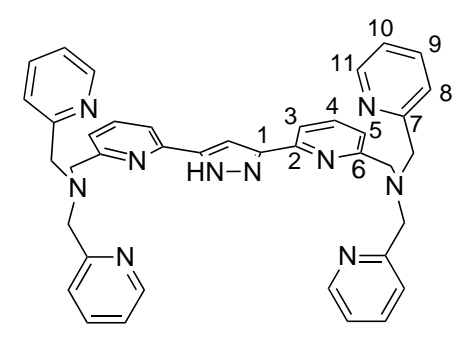

$\left(\mathrm{ddd},{ }^{3,4} J_{\mathrm{H}, \mathrm{H}}=7.6,4.9,0.9 \mathrm{~Hz}, 4 \mathrm{H}, 11-\mathrm{H}^{\mathrm{Py}}\right), 3.89\left(\mathrm{~s}, 8 \mathrm{H}, \mathrm{CH}_{2}\right), 3.86\left(\mathrm{~s}, 4 \mathrm{H}, \mathrm{CH}_{2}\right)$.

${ }^{13} \mathbf{C}\left\{{ }^{1} \mathbf{H}\right\}$-NMR $\left(126 \mathrm{MHz}, 298 \mathrm{~K}, \mathrm{MeCN}_{3}\right) \delta(\mathrm{ppm})=160.2\left(\mathrm{C} 7^{\mathrm{Py}}\right), 159.2\left(\mathrm{C} 2 / 6^{\mathrm{Py}}\right)$, $149.8\left({\mathrm{C} 11^{\mathrm{Py}}}^{\mathrm{P}}\right), 138.3\left(\mathrm{C}^{\mathrm{Py}}\right), 137.5\left(\mathrm{C}^{\mathrm{Py}}\right), 124.1\left(\mathrm{C}^{\mathrm{Py}}\right), 123.2\left(\mathrm{C}^{\mathrm{Py}}\right), 122.9\left(\mathrm{C}^{\mathrm{Py}}\right)$, $118.9\left(\mathrm{C} 2{ }^{\mathrm{Py}}\right), 102.1\left(\mathrm{C}^{\mathrm{Pz}}\right), 60.6\left(\mathrm{CH}_{2}\right), 59.6\left(\mathrm{CH}_{2}\right)$. ( $\mathrm{C}^{\mathrm{Pz}}$ not observed).

MS $\left(\mathbf{E S I}^{+}, \mathbf{M e O H} / \mathrm{MeCN}\right): \mathrm{m} / \mathrm{z}(\%)=667(100)[\mathrm{M}+\mathrm{Na}]^{+}, 706(35)[\mathrm{M}+\mathrm{Na}+\mathrm{K}]^{+}$.

IR(ATR, solid) $1589(\mathrm{~s}), 1568(\mathrm{~s}), 1471(\mathrm{~m}), 1432(\mathrm{~m}), 1364(\mathrm{~m}), 1299(\mathrm{~m}), 1265(\mathrm{w})$, $1245(\mathrm{w}), 1217(\mathrm{w}), 1182(\mathrm{w}), 1148(\mathrm{~s}), 1121(\mathrm{~m}), 1081(\mathrm{~m}), 1047(\mathrm{~s}), 992(\mathrm{~s}), 890(\mathrm{w})$, $836(\mathrm{w}), 798(\mathrm{~s}), 751(\mathrm{~m})$.

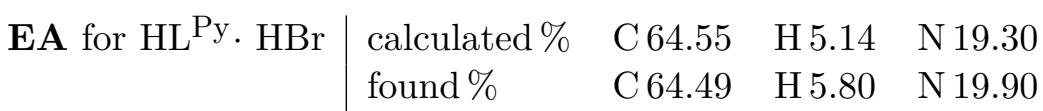

\subsection{Complex Formation with Actetonitrile Coligands}

\section{Synthesis of $\left[L^{\operatorname{lm}} \mathrm{Fe}\{\mathrm{Fe}(\mathrm{MeCN}\}](\mathrm{OTf})_{3}\right.$ (1a)}

$\mathrm{HL}^{\mathrm{Im}}$ (50.0 mg, $0.078 \mathrm{mmol}, 1.00 \mathrm{eq}$.) was dissolved in dry, degassed MeCN and stirred over activated $3 \AA$ molecular sieves for $10 \mathrm{~h}$. The solution was filtered and $\mathrm{NaO} t \mathrm{Bu}$ (7.31 mg, $0.08 \mathrm{mmol}, 1.00$ eq.) and $\mathrm{Fe}(\mathrm{OTf})_{2} \cdot 2 \mathrm{MeCN}$ (66.8 $\mathrm{mg} 0.152 \mathrm{mmol}, 2.00$ eq.) were added. The reaction mixture was stirred another $2 \mathrm{~h}$. Filtration and slow diffusion of diethyl ether into the filtrate afforded red single crystals suitable for x-ray diffraction.

MS (ESI $\left.{ }^{+}, \mathbf{M e C N}\right): \mathrm{m} / \mathrm{z}(\%)=401(100)\left[\mathrm{L}^{\mathrm{Im}_{\mathrm{Fe}} \mathrm{Cl}}\right]^{2+}$.

IR(ATR, solid) $1608(\mathrm{~m}), 1572(\mathrm{~m}), 1502(\mathrm{~s}), 1458(\mathrm{~m}), 1255(\mathrm{~m}), 1221(\mathrm{~m}), 1144(\mathrm{~m})$, $1028(\mathrm{~s}), 958(\mathrm{~m}), 796(\mathrm{~m}), 746(\mathrm{~m}), 633(\mathrm{~s}), 570(\mathrm{~s}), 517(\mathrm{~s})$.

\begin{tabular}{l|llll} 
EA for $\left[\mathrm{L}^{\operatorname{Im}} \mathrm{Fe}\{\mathrm{Fe}(\mathrm{MeCN}\}]^{3+}\right.$, & calculated \% & $\mathrm{C} 34.49$ & $\mathrm{H} 2.96$ & $\mathrm{~N} 14.72$ \\
3 OTf $, \mathrm{Na}(\mathrm{OTf}), \mathrm{MeCN}$ & found \% & $\mathrm{C} 34.91$ & $\mathrm{H} 2.93$ & $\mathrm{~N} 14.39$.
\end{tabular}

UV/Vis (EtCN, 21 $\left.{ }^{\circ} \mathrm{C}\right) \lambda_{\max }[\mathrm{nm}]\left(\epsilon_{\mathrm{rel}}\left[\mathrm{M}^{-1} \mathrm{~cm}^{-1}\right]\right) 259$ (24932), 303 (20676), 419 (1535). 


\section{Synthesis of $\left[L^{\operatorname{Im}} \mathrm{Fe}\{\mathrm{Fe}(\mathrm{MeCN}\}]\left(\mathrm{BF}_{4}\right)_{3}(\mathbf{1 b})\right.$}

In a glovebox $\mathrm{HL}^{\mathrm{Im}}(50.0 \mathrm{mg}, 0.08 \mathrm{mmol}, 1.00$ eq.) was dissolved in dry MeCN and stirred over activated $3 \AA$ molecular sieves for $10 \mathrm{~h}$. The solution was filtered and $\mathrm{NaO} t \mathrm{Bu}$ $(7.45 \mathrm{mg}, 0.08 \mathrm{mmol}, 1.00 \mathrm{eq}$.$) and \left[\mathrm{Fe}(\mathrm{MeCN})_{6}\right]\left(\mathrm{BF}_{4}\right)_{2}(73.8 \mathrm{mg}, 0.16 \mathrm{mmol}, 2.00 \mathrm{eq}$. was added. The reaction mixture was stirred another $2 \mathrm{~h}$. Filtration and slow diffusion of diethyl ether into the filtrate afforded red single crystals suitable for x-ray diffraction.

MS $\left(\mathbf{E S I}{ }^{+}, \mathbf{M e C N}\right): \mathrm{m} / \mathrm{z}(\%)=255(30)\left[\mathrm{L}^{\mathrm{Im}_{\mathrm{Fe}}}\right]^{3+}, 275(70), 401(100)\left[\mathrm{L}^{\mathrm{Im}} \mathrm{Fe}_{2} \mathrm{Cl}\right]^{2+}$.

\section{Synthesis of $\left[\mathrm{L}^{\mathrm{Py}}\left\{\mathrm{Fe}(\mathrm{MeCN}\}_{2}\right](\mathrm{OTf})_{3}\right.$ (2a)}

In a glovebox $\mathrm{HL}^{\mathrm{Py}} \cdot \mathrm{HBr}(50.0 \mathrm{mg}, 0.07 \mathrm{mmol}, 1.00 \mathrm{eq}$.) was dissolved in dry MeCN and stirred over activated $3 \AA$ molecular sieves for $10 \mathrm{~h}$. The solution was filtered and $\mathrm{NaO} t \mathrm{Bu}$ (7.45 mg, $0.08 \mathrm{mmol}, 1.00$ eq.) and $\mathrm{Fe}(\mathrm{OTf})_{2} \cdot 2 \mathrm{MeCN}$ (68.2 mg, $0.16 \mathrm{mmol}, 2.00$ eq.) was added. The reaction mixture was stirred another $2 \mathrm{~h}$. Filtration and slow diffusion of diethyl ether into the filtrate afforded red single crystals suitable for x-ray diffraction.

MS (ESI $\left.{ }^{+}, \quad \mathbf{M e C N}\right): \mathrm{m} / \mathrm{z} \quad(\%)=251(10)\left[\mathrm{L}^{\mathrm{Py}_{\mathrm{Fe}}}\right]^{3+}, \quad 390(90)\left[\mathrm{L}^{\mathrm{Py}} \mathrm{Fe}_{2}(\mathrm{CN})\right]^{2+}$, $407(100)\left[\mathrm{L}^{\mathrm{Py}} \mathrm{Fe}_{2}\left(\mathrm{H}_{2} \mathrm{O}\right)(\mathrm{MeCN})\right]^{2+}, 414(80)\left[\mathrm{L}^{\mathrm{Py}} \mathrm{Fe}_{2}\left(\mathrm{O}^{t} \mathrm{Bu}\right)\right]^{2+}$.

IR(ATR, solid) 1608 (s), 1569 (s), 1534 (s), 1464 (s), 1441 (d), 1251 (m), 1222 (s), 1150 (d), $1027(\mathrm{~s}), 896(\mathrm{w}), 871(\mathrm{~m}), 813(\mathrm{~s}), 764(\mathrm{~s}), 637(\mathrm{~s}), 572(\mathrm{~s}), 515(\mathrm{~s})$.

$$
\text { EA for }\left[\mathrm{L}^{\mathrm{Py}}\left\{\mathrm{Fe}(\mathrm{MeCN}\}_{2}\right]^{3+}, 3 \text { OTf } \mid \begin{array}{llll}
\text { Calculated \% } & \mathrm{C} 43.00 & \mathrm{H} 3.22 & \mathrm{~N} 13.08 \\
\text { Found \% } & \mathrm{C} 43.01 & \text { H3.30 } & \text { N } 12.82
\end{array}\right.
$$

UV/Vis (EtCN, 21 $\left.{ }^{\circ} \mathrm{C}\right) \lambda_{\max }[\mathrm{nm}]\left(\epsilon_{\mathrm{rel}}\left[\mathrm{M}^{-1} \mathrm{~cm}^{-1}\right]\right) 254$ (31843), 296 (18300), 385 (9420).

\section{Synthesis of $\left[\mathrm{L}^{\mathrm{Py}}\left\{\mathrm{Fe}(\mathrm{MeCN}\}_{2}\right]\left(\mathrm{BF}_{4}\right)(2 \mathrm{~b})\right.$}

In a glovebox $\mathrm{HL}^{\mathrm{Py}} \cdot \mathrm{HBr}(50.0 \mathrm{mg}, 0.07 \mathrm{mmol}, 1.00$ eq. $)$ was dissolved in dry, degassed MeCN and stirred over activated $3 \AA$ molecular sieves for $12 \mathrm{~h}$. The suspension was filtered and $\mathrm{NaO} t \mathrm{Bu}(6.62 \mathrm{mg}, 0.07 \mathrm{mmol}, 1.00 \mathrm{eq}$.$) and \left[\mathrm{Fe}(\mathrm{MeCN})_{6}\right]\left(\mathrm{BF}_{4}\right)_{2}(65.6 \mathrm{mg}$, $0.14 \mathrm{mmol}, 2.00$ eq.) was added. The reaction mixture was stirred for another $2 \mathrm{~h}$ and then filtered again. Diffusion of diethyl ether into the filtrate afforded red single crystals suitable for x-ray diffraction.

MS $\left(\mathbf{E S I}^{+}, \mathbf{M e C N}\right): \mathrm{m} / \mathrm{z}(\%)=251(100)\left[\mathrm{L}^{\mathrm{Py}} \mathrm{Fe}_{2}\right]^{3+}, 390(65)\left[\mathrm{L}^{\mathrm{Py}_{\mathrm{Fe}}}+\mathrm{CN}\right]^{2+}$, $407(40), 422(20)\left[\mathrm{L}^{\mathrm{Py}} \mathrm{Fe}_{2}+2 \mathrm{BF}_{4}+\mathrm{H}\right]^{2+}, 456(50), 477(20), 491(22), 505(60)\left[\mathrm{L}^{\mathrm{Py}} \mathrm{Fe}_{2}+\right.$ $\left.2 \mathrm{BF}_{4}+2 \mathrm{MeCN}\right]^{2+}$ (several signals were not assignable, but reproducible).

IR(ATR, solid) $1606(\mathrm{~s}), 1569(\mathrm{~s}), 1535(\mathrm{~s}), 1501(\mathrm{~m}), 1460(\mathrm{~s}), 1438(\mathrm{~m}), 1424(\mathrm{w})$, $1373(\mathrm{w}), 1305(\mathrm{~s}), 1272(\mathrm{w}), 1223(\mathrm{~m}), 1158(\mathrm{~m}), 1028(\mathrm{~m}), 903(\mathrm{~m}), 871(\mathrm{~m}), 790(\mathrm{~s})$, 
$770(\mathrm{w}), 637(\mathrm{~m}), 572(\mathrm{~m}), 518(\mathrm{~s})$.

\subsection{Diiron Nitrosyl Complexes and their Reduction Products}

\section{Synthesis of $\left[\mathrm{L}^{\mathrm{Im}}\{\mathrm{Fe}(\mathrm{NO})\}_{2}\right](\mathrm{OTf})_{3}(4)$}

Single crystals of $\left[\mathrm{L}^{\mathrm{Im}} \mathrm{Fe}\{\mathrm{Fe}(\mathrm{MeCN}\}](\mathrm{OTf})_{3}\right.$ 1a were dried overnight and dissolved in dry methanol. On a Schlenk line with $\mathrm{NO}$ gas setup, $\mathrm{NO}_{(\mathrm{g})}$ was added to the complex solution, which instantly turned from light red to dark brown. Crystallization by diethyl ether diffusion into the reaction mixture under $\mathrm{NO}_{(\mathrm{g})}$ atmosphere afforded dark brown single crystals suitable for x-ray diffraction after a few days.

MS (ESI $\left.{ }^{+}, \mathbf{M e O H}\right): \mathrm{m} / \mathrm{z}(\%)=413(100)\left[\mathrm{L}^{\mathrm{Im}} \mathrm{Fe}_{2}(\mathrm{OAc})\right]^{2+}, 885(20)\left[\mathrm{L}^{\mathrm{Im}} \mathrm{Fe}_{2}(\mathrm{OAc})_{2}\right]^{+}$, $975(60)\left[\left[\mathrm{L}^{\mathrm{Im}} \mathrm{Fe}_{2}(\mathrm{OAc})(\mathrm{OTf})\right]^{+}\right.$(bound NO is lost, OAc from spectrometer).

IR(ATR, solid) $1760(\mathrm{~m})$ [NO-stretch], $1732 /(\mathrm{m})[$ NO-stretch], $1605(\mathrm{~m}), \quad 1576(\mathrm{~m})$, $1506(\mathrm{~m}), 1463(\mathrm{~s}), 1427(\mathrm{~m}), 1256(\mathrm{~m}), 1220(\mathrm{~m}), 1143(\mathrm{w}), 1085(\mathrm{~m}), 1027(\mathrm{~s}), 954(\mathrm{~m})$, $902(\mathrm{~m}), 879(\mathrm{~m}), 797(\mathrm{~m}), 771(\mathrm{~m}), 751(\mathrm{~m}), 634(\mathrm{~s}), 571(\mathrm{~m}), 515(\mathrm{~s})$.

$$
\text { EA for } \left.\left[\mathrm{L}^{\operatorname{Im}}\{\mathrm{Fe}(\mathrm{NO})\}_{2}\right](\mathrm{OTf})_{3}\right), \mathrm{MeOH} \mid \begin{array}{llll}
\text { calculated \% } & \text { C } 35.85 & \text { H } 3.32 & \text { N } 17.15 \\
\text { found \% } & \text { C } 36.17 & \text { H } 3.17 & \text { N } 17.16 .
\end{array}
$$

UV/Vis (DCM, 25 $\left.{ }^{\circ} \mathrm{C}\right) \lambda_{\max }[\mathrm{nm}]\left(\epsilon_{\mathrm{rel}}\left[\mathrm{M}^{-1} \mathrm{~cm}^{-1}\right]\right) 296(43740), \mathrm{sh} \sim 460(3350)$.

\section{Synthesis of $\left[\mathrm{L}^{\mathrm{Py}}\left\{\mathrm{Fe}(\mathrm{NO}\}_{2}\right](\mathrm{OTf})_{3}\right.$ (5)}

Single crystals of $\left[\mathrm{L}^{\mathrm{Py}}\{\mathrm{Fe}(\mathrm{MeCN})\}_{2}\right](\mathrm{OTf})_{3}$ 2a were dissolved in dry acetonitrile. On a Schlenk line with $\mathrm{NO}$ gas setup, $\mathrm{NO}_{(\mathrm{g})}$ was added to the reaction mixture, which slowly turned from dark red to brown. Crystallization by diethyl ether diffusion into the reaction mixture under $\mathrm{NO}_{(\mathrm{g})}$ atmosphere afforded dark brown single crystals suitable for x-ray diffraction after a few days.

MS (ESI $\left.{ }^{+}, \mathbf{M e O H}\right): \mathrm{m} / \mathrm{z}(\%)=386(100)\left[\mathrm{L}^{\mathrm{Py}} \mathrm{Fe}_{2}(\mu-\mathrm{OH})\right]^{2+}, 390(50)\left[\mathrm{L}^{\mathrm{Py}} \mathrm{Fe}_{2}(\mathrm{CN})\right]^{2+}$, $422(10), 452(20)\left[\mathrm{L}^{\mathrm{Py}} \mathrm{Fe}_{2}(\mathrm{OTf})\right]^{2+}, 505$ (15).

IR(ATR, solid) 1778 (w) [NO-stretch], 1606 (s), $1578(\mathrm{~m}), 1464(\mathrm{~m}), 1444(\mathrm{w}), 1257$ (m), $1223(\mathrm{~s}), 1140(\mathrm{w}), 1098(\mathrm{~m}), 1026(\mathrm{~s}), 904(\mathrm{~m}), 883(\mathrm{~m}), 797(\mathrm{~m}), 770(\mathrm{~m}), 634(\mathrm{~s}), 571(\mathrm{~s})$, $515(\mathrm{~s})$.

UV/Vis (DCM/MeOH 5:1, 24.5 $\left.{ }^{\circ} \mathrm{C}\right) \lambda_{\max }[\mathrm{nm}]\left(\epsilon_{\mathrm{rel}}\left[\mathrm{M}^{-1} \mathrm{~cm}^{-1}\right]\right) 262(29075), 298$ (14120), 439 (1160). 


\section{Formation of $\left[\mathrm{L}^{\operatorname{lm}} \mathrm{Fe}_{2}(\mu-\mathrm{OMe})\right](\mathrm{OTf})_{2}\left(4^{\mathrm{Red}}\right)$}

Single crystals of $\left[\mathrm{L}^{\operatorname{Im}}\{\mathrm{Fe}(\mathrm{NO})\}_{2}\right](\mathrm{OTf})_{3} 4$ were dissolved in dry, degassed methanol in a glovebox. To this solution $\mathrm{Cp}_{2} \mathrm{Co}$ (excess) was added and the reaction mixture was stirred for $1 \mathrm{~h}$. The resulting suspension was filtered. Slow diffusion of diethyl ether into the filtrate afforded single crystals suitable for x-ray diffraction.

IR(ATR, solid) 1602 (s), 1567 (s), 1499 (s), $1449(\mathrm{~m}), 1423(\mathrm{~s}), 1362(\mathrm{w}), 1263(\mathrm{~s}), 1221$ (s), $1157(\mathrm{w}), 1127(\mathrm{~m}), 1080(\mathrm{~s}), 1061(\mathrm{~s}), 1029(\mathrm{~s}), 956(\mathrm{~m}), 878(\mathrm{~m}), 806(\mathrm{~s}), 772(\mathrm{~s}), 750(\mathrm{~s})$, $735(\mathrm{~s}), 680(\mathrm{~s}), 634(\mathrm{~s}), 571(\mathrm{~s}), 517(\mathrm{~s})$.

\section{Formation of $\left[\mathrm{L}^{\mathrm{Py}} \mathrm{Fe}_{2}(\mu-\mathrm{MeO}-\mathrm{HOMe})\right](\mathrm{OTf})_{2}\left(5^{\mathrm{Red}}\right)$}

Single crystals of $\left[\mathrm{L}^{\mathrm{Py}}\{\mathrm{Fe}(\mathrm{NO})\}_{2}\right](\mathrm{OTf})_{3} \mathbf{5}$ were dissolved in dry, degassed methanol in a glovebox. To this solution $\mathrm{Cp}_{2} \mathrm{Co}$ (excess) was added and the reaction mixture was stirred for $1 \mathrm{~h}$. The solution was filtered. Slow diffusion of diethyl ether into the filtrate afforded single crystals suitable for x-ray diffraction.

IR(ATR, solid) $1601(\mathrm{~s}), 1572(\mathrm{~s}), 1526(\mathrm{w}), 1478(\mathrm{w}), 1459(\mathrm{~m}), 1441(\mathrm{~m}), 1355(\mathrm{w})$, $1260(\mathrm{~s}), 1221(\mathrm{~s}), 1151(\mathrm{~s}), 1102(\mathrm{~m}), 1049(\mathrm{~m}), 1027(\mathrm{~s}), 959(\mathrm{~m}), 903(\mathrm{w}), 879(\mathrm{w})$, $808(\mathrm{~m}), 763(\mathrm{~m}), 698(\mathrm{~m}), 636(\mathrm{~s}), 570(\mathrm{~s}), 514(\mathrm{~s})$.

\subsection{Complexes with $\mathrm{K}_{5} \mathrm{~L} \mathrm{COO}$}

\section{Synthesis of $\left[\mathrm{L}^{\mathrm{COO}}\{\mathrm{Fe}(\mathrm{DMF})\}_{2}(\mu-\mathrm{OH})\right](6)$}

$\mathrm{K}_{5} \mathrm{~L}^{\mathrm{COO}} 1$ (100 mg, $0.14 \mathrm{mmol}, 1.00 \mathrm{eq}$.) was dissolved in demineralized water and $\mathrm{Fe}\left(\mathrm{BF}_{4}\right)_{2} \cdot 7 \mathrm{H}_{2} \mathrm{O}$ (91.4 mg, $0.27 \mathrm{mmol}, 2.00$ eq.) was added. The reaction mixture was stirred for $2 \mathrm{~h}$. The water was removed in vacuo. The residue was dissolved in dimethylformamide. Subsequently the reaction mixture was filtered. Diffusion of diethyl ether into the reaction mixture afforded single crystals suitable for x-ray diffraction.

MS (ESI $\left.{ }^{+}, \mathbf{M e O H}\right): \mathrm{m} / \mathrm{z}(\%)=659(100)\left[\mathrm{L}^{\mathrm{COO}} \mathrm{Fe}_{2}(\mu-\mathrm{OH})+\mathrm{Na}\right]^{+}, 681(60)$.

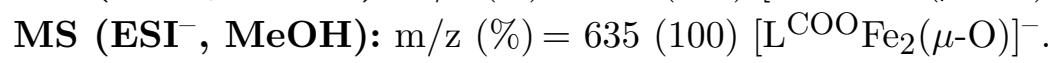

\section{Synthesis of $\left[\mathrm{LCO}^{\mathrm{CO}}\left\{\mathrm{Fe}\left(\mathrm{H}_{2} \mathrm{O}\right)\right\}_{2}(\mu-\mathrm{OH})\right](7)$}

$\mathrm{K}_{5} \mathrm{~L}^{\mathrm{COO}} 1$ (50 mg, $0.07 \mathrm{mmol}, 1.00$ eq.) was dissolved in demineralized water and $\mathrm{Fe}\left(\mathrm{BF}_{4}\right)_{2} \cdot 7 \mathrm{H}_{2} \mathrm{O}$ (45.7 mg, $0.14 \mathrm{mmol}, 2.00$ eq. $)$ was added. The reaction mixture was stirred for a few minutes and was quickly filtered. Diffusion of acetone into the reaction mixture afforded single crystals suitable for x-ray diffraction (28.0 mg, $0.03 \mathrm{mg}, 48 \%$ ).

\footnotetext{
${ }^{1}$ Provided by S. Fischer 105
} 
MS $\left(\mathbf{E S I}^{+}, \boldsymbol{i} \mathbf{P r O H} / \mathbf{H}_{2} \mathbf{O}\right): \mathrm{m} / \mathrm{z}(\%)=659(100)\left[\mathrm{L}^{\mathrm{COO}} \mathrm{Fe}_{2}(\mu-\mathrm{OH})+\mathrm{Na}\right]^{+}, 675(30)$ $\left[\mathrm{L}^{\mathrm{COO}} \mathrm{Fe}_{2}(\mu-\mathrm{OH})+\mathrm{K}\right]^{+}, 681(60)$.

MS $\left(\right.$ ESI $\left.^{-}, \boldsymbol{i} \mathbf{P r O H} / \mathbf{H}_{2} \mathbf{O}\right): \mathrm{m} / \mathrm{z}(\%)=635(100)\left[\mathrm{L}^{\mathrm{COO}_{\mathrm{Fe}}}(\mu-\mathrm{O})\right]^{-}$.

\section{Synthesis of $\mathrm{K}_{2}\left[\mathrm{~L}^{\mathrm{COO}} \mathrm{Mn}_{4}\right](8)$}

$\mathrm{K}_{5} \mathrm{~L}^{\mathrm{COO}} 1$ (50.0 mg, $0.07 \mathrm{mmol}, 1.00$ eq.) was dissolved in a 1:1 mixture of methanol and water. To this solution $\mathrm{Mn}(\mathrm{OAc})_{2}(33.0 \mathrm{mg}, 0.13 \mathrm{mmol}, 2.00 \mathrm{eq}$.) was added. The pale yellow reaction mixture was stirred for $2 \mathrm{~h}$ and filtered. Diffusion of diethyl ether into the reaction mixture yielded pale yellow crystals suitable for x-ray diffraction.

MS $\left(\mathbf{E S I}^{-}, \mathbf{H}_{2} \mathbf{O} / \mathbf{M e O H}\right): \mathrm{m} / \mathrm{z}(\%)=564(80)[\mathrm{LMn}]^{-}, 617(100)\left[\mathrm{L}^{\mathrm{COO}} \mathrm{Mn}_{2}\right]^{-}$.

IR $(\mathrm{KBr}$, solid) $2964(\mathrm{w}), 2915(\mathrm{w}), 2844(\mathrm{w}), 1603(\mathrm{~s}), 1569(\mathrm{~s}), 1463(\mathrm{~m}), 1435(\mathrm{~m})$, $1395(\mathrm{~m}), 1312(\mathrm{~s}), 1237(\mathrm{~s}), 1184(\mathrm{~s}), 1126(\mathrm{~s}), 983(\mathrm{~s}), 918(\mathrm{w}), 811(\mathrm{~m}), 784(\mathrm{~m}), 744(\mathrm{~m})$, $649(\mathrm{w}), 533(\mathrm{w})$. 



\section{Crystallographic Details}

Table 10.1: Crystal data and refinement details for $\mathbf{1 a}$ and $\mathbf{1 b}$.

\begin{tabular}{|c|c|c|}
\hline compound & $1 \mathrm{a}$ & $1 \mathrm{~b}$ \\
\hline empirical formula & $\mathrm{C}_{42} \mathrm{H}_{45} \mathrm{~F}_{9} \mathrm{Fe}_{2} \mathrm{~N}_{16} \mathrm{O}_{9} \mathrm{~S}_{3}$ & $\mathrm{C}_{37} \mathrm{H}_{42} \mathrm{~B}_{3} \mathrm{~F}_{12} \mathrm{Fe}_{2} \mathrm{~N}_{15}$ \\
\hline formula weight & 1296.82 & 1068.98 \\
\hline $\mathrm{T}[\mathrm{K}]$ & $133(2)$ & $133(2)$ \\
\hline crystal size $\left[\mathrm{mm}^{3}\right]$ & $0.3 \times 0.14 \times 0.06$ & $0.431 \times 0.339 \times 0.139$ \\
\hline crystal system & monoclinic & triclinic \\
\hline space group & $P 2_{1} / \mathrm{n}$ & $P \overline{1}$ \\
\hline $\mathrm{a}[\AA]$ & $14.236(3)$ & $12.151(2)$ \\
\hline $\mathrm{b}[\AA]$ & $13.324(3)$ & $15.010(3)$ \\
\hline$c[\AA]$ & $27.747(6)$ & $16.038(3)$ \\
\hline$\alpha\left[^{\circ}\right]$ & 90 & $111.90(3)$ \\
\hline$\beta\left[^{\circ}\right]$ & $91.91(3)$ & $108.32(3)$ \\
\hline$\gamma\left[^{\circ}\right]$ & 90 & $93.79(3)$ \\
\hline $\mathrm{V}\left[\AA^{3}\right]$ & $5260.3(18)$ & $2519.8(11)$ \\
\hline $\mathrm{Z}$ & 4 & 2 \\
\hline$\rho\left[\mathrm{g} / \mathrm{cm}^{3}\right]$ & 1.637 & 1.409 \\
\hline $\mathrm{F}(000)$ & 2648 & 1088 \\
\hline$\mu\left[\mathrm{mm}^{-1}\right]$ & 0.772 & 0.664 \\
\hline $\mathrm{T}_{\min } / \mathrm{T}_{\max }$ & $0.8630 / 0.9604$ & $-/-$ \\
\hline$\theta$-range $\left[{ }^{\circ}\right]$ & $1.469-25.642$ & $1.471-26.762$ \\
\hline \multirow[t]{3}{*}{ hkl-range } & $-17 \leq \mathrm{h} \leq 15$ & $-15 \leq \mathrm{h} \leq 15$ \\
\hline & $-16 \leq \mathrm{k} \leq 16$ & $-18 \leq \mathrm{k} \leq 18$ \\
\hline & $-33 \leq 1 \leq 33$ & $-20 \leq 1 \leq 20$ \\
\hline measured refl. & 47058 & 25786 \\
\hline unique refl. $\left[\mathrm{R}_{\text {int }}\right]$ & $9922[0.1381]$ & $10638[0.0462]$ \\
\hline observed refl. $(\mathrm{I}>2 \sigma(\mathrm{I}))$ & 5168 & 7461 \\
\hline data / restraints / param. & $9922 / 57 / 761$ & $10638 / 10 / 673$ \\
\hline goodness-of-fit $\left(\mathrm{F}^{2}\right)$ & 0.989 & 1.051 \\
\hline $\mathrm{R} 1, \mathrm{wR} 2(\mathrm{I}>2 \sigma(\mathrm{I}))$ & $0.0783,0.1432$ & $0.0587,0.1578$ \\
\hline R1, wR2 (all data) & $0.1606,0.1717$ & $0.0829,0.1705$ \\
\hline resid. el. dens. $\left[\mathrm{e} / \AA^{3}\right]$ & $-0.392 / 0.702$ & $-0.550 / 0.828$ \\
\hline
\end{tabular}


Table 10.2: Crystal data and refinement details for $\mathbf{2 a}$ and $\mathbf{2} \mathbf{b}$.

\begin{tabular}{|c|c|c|}
\hline compound & $2 \mathbf{a}$ & $2 \mathrm{~b}$ \\
\hline empirical formula & $\mathrm{C}_{49} \mathrm{H}_{45.50} \mathrm{~F}_{9} \mathrm{Fe}_{2} \mathrm{~N}_{13.50} \mathrm{O}_{9.25} \mathrm{~S}_{3}$ & $\mathrm{C}_{43} \mathrm{H}_{41} \mathrm{~B}_{3} \mathrm{~F}_{12} \mathrm{Fe}_{2} \mathrm{~N}_{12}$ \\
\hline formula weight & 1350.37 & 1098.01 \\
\hline $\mathrm{T}[\mathrm{K}]$ & $133(2)$ & $133(2)$ \\
\hline crystal size $\left[\mathrm{mm}^{3}\right]$ & $0.23 \times 0.17 \times 0.13$ & $0.270 \times 0.170 \times 0.150$ \\
\hline crystal system & triclinic & monoclinic \\
\hline space group & $P \overline{1}$ & $P 2_{1} / \mathrm{n}$ \\
\hline $\mathrm{a}[\AA]$ & $13.4989(5)$ & $13.0815(5)$ \\
\hline $\mathrm{b}[\AA]$ & $17.1796(6)$ & $20.8318(6)$ \\
\hline $\mathrm{c}[\AA]$ & $24.3682(10)$ & $16.5757(6)$ \\
\hline$\alpha\left[^{\circ}\right]$ & $93.662(3)$ & 90 \\
\hline$\beta\left[^{\circ}\right]$ & $92.759(3)$ & $95.137(3)$ \\
\hline$\gamma\left[^{\circ}\right]$ & $93.197(3)$ & 90 \\
\hline $\mathrm{V}\left[\AA^{3}\right]$ & $5623.2(4)$ & $4498.9(3)$ \\
\hline $\mathrm{Z}$ & 4 & 4 \\
\hline$\rho\left[\mathrm{g} / \mathrm{cm}^{3}\right]$ & 1.595 & 1.621 \\
\hline $\mathrm{F}(000)$ & 2756 & 2232 \\
\hline$\mu\left[\mathrm{mm}^{-1}\right]$ & 0.725 & 0.744 \\
\hline $\mathrm{T}_{\min } / \mathrm{T}_{\max }$ & $0.8486 / 0.9361$ & $0.8190 / 0.9352$ \\
\hline$\theta$-range $\left[^{\circ}\right]$ & $1.19-25.70$ & $1.574-26.740$ \\
\hline \multirow[t]{3}{*}{ hkl-range } & $-16 \leq \mathrm{h} \leq 16$ & $-16 \leq \mathrm{h} \leq 16$ \\
\hline & $-20 \leq \mathrm{k} \leq 20$ & $-26 \leq \mathrm{k} \leq 26$ \\
\hline & $-29 \leq 1 \leq 29$ & $-20 \leq 1 \leq 17$ \\
\hline measured refl. & 47094 & 45705 \\
\hline unique refl. $\left[\mathrm{R}_{\text {int }}\right]$ & $20940[0.0625]$ & $9549[0.0813]$ \\
\hline observed refl. $(\mathrm{I}>2 \sigma(\mathrm{I}))$ & 12768 & 6950 \\
\hline data / restraints / param. & $20940 / 0 / 1556$ & $9549 / 10 / 667$ \\
\hline goodness-of-fit $\left(\mathrm{F}^{2}\right)$ & 0.990 & 1.123 \\
\hline $\mathrm{R} 1, \mathrm{wR} 2(\mathrm{I}>2 \sigma(\mathrm{I}))$ & $0.0622,0.0979$ & $0.0603,0.1204$ \\
\hline R1, wR2 (all data) & $0.1194,0.1127$ & $0.0914,0.1300$ \\
\hline resid. el. dens. $\left[\mathrm{e} / \AA^{3}\right]$ & $-0.521 / 0.577$ & $-0.482 / 0.851$ \\
\hline
\end{tabular}


Table 10.3: Crystal data and refinement details for 3 and 4 .

\begin{tabular}{|c|c|c|}
\hline compound & 3 & 4 \\
\hline empirical formula & $\mathrm{C}_{46} \mathrm{H}_{64} \mathrm{~F}_{12} \mathrm{Fe}_{2} \mathrm{~N}_{14} \mathrm{O}_{18.50} \mathrm{~S}_{4}$ & $\mathrm{C}_{39} \mathrm{H}_{42} \mathrm{~F}_{9} \mathrm{Fe}_{2} \mathrm{~N}_{16} \mathrm{O}_{12} \mathrm{~S}_{3}$ \\
\hline formula weight & 1577.05 & 1305.76 \\
\hline $\mathrm{T}[\mathrm{K}]$ & $133(2)$ & $133(2)$ \\
\hline crystal size $\left[\mathrm{mm}^{3}\right]$ & $0.500 \times 0.290 \times 0.120$ & $0.310 \times 0.290 \times 0.230$ \\
\hline crystal system & triclinic & triclinic \\
\hline space group & $P \overline{1}$ & $P \overline{1}$ \\
\hline $\mathrm{a}[\AA]$ & $13.260(3)$ & $13.1425(18)$ \\
\hline $\mathrm{b}[\AA]$ & $15.248(3)$ & $13.7958(14)$ \\
\hline$c[\AA]$ & $17.172(3)$ & $16.3646(13)$ \\
\hline$\alpha\left[^{\circ}\right]$ & $105.06(3)$ & $87.514(7)$ \\
\hline$\beta\left[^{\circ}\right]$ & $93.84(3)$ & $74.559(9)$ \\
\hline$\gamma\left[^{\circ}\right]$ & $97.45(3)$ & $66.343(8)$ \\
\hline $\mathrm{V}\left[\AA^{3}\right]$ & $3306.0(12)$ & $2612.9(5)$ \\
\hline $\mathrm{Z}$ & 2 & 2 \\
\hline$\rho\left[\mathrm{g} / \mathrm{cm}^{3}\right]$ & 1.584 & 1.660 \\
\hline $\mathrm{F}(000)$ & 1620 & 1330 \\
\hline$\mu\left[\mathrm{mm}^{-1}\right]$ & 0.676 & 0.782 \\
\hline $\mathrm{T}_{\min } / \mathrm{T}_{\max }$ & $0.7287 / 0.9220$ & $0.7292 / 0.8492$ \\
\hline$\theta$-range $\left[^{\circ}\right]$ & $1.399-26.778$ & $1.616-26.739$ \\
\hline \multirow[t]{3}{*}{ hkl-range } & $-15 \leq \mathrm{h} \leq 16$ & $-16 \leq \mathrm{h} \leq 16$ \\
\hline & $-19 \leq \mathrm{k} \leq 19$ & $-17 \leq \mathrm{k} \leq 17$ \\
\hline & $-21 \leq 1 \leq 21$ & $-20 \leq 1 \leq 20$ \\
\hline measured refl. & 34909 & 28380 \\
\hline unique refl. $\left[\mathrm{R}_{\text {int }}\right]$ & $13993[0.0583]$ & $11051[0.0252]$ \\
\hline observed refl. $(\mathrm{I}>2 \sigma(\mathrm{I}))$ & 10604 & 9193 \\
\hline data / restraints / param. & $13993 / 0 / 877$ & $11051 / 60 / 727$ \\
\hline goodness-of-fit $\left(\mathrm{F}^{2}\right)$ & 1.039 & 1.013 \\
\hline $\mathrm{R} 1, \mathrm{wR} 2(\mathrm{I}>2 \sigma(\mathrm{I}))$ & $0.0531,0.1390$ & $0.0564,0.1466$ \\
\hline R1, wR2 (all data) & $0.0735,0.1483$ & $0.0679,0.1539$ \\
\hline resid. el. dens. $\left[\mathrm{e} / \AA^{3}\right]$ & $-0.798 / 1.071$ & $-0.866 / 0.918$ \\
\hline
\end{tabular}


Table 10.4: Crystal data and refinement details for $\mathbf{4}^{\text {Red }}$ and $\mathbf{5}$ (picture only).

\begin{tabular}{|c|c|c|}
\hline compound & $4^{\text {Red }}$ & 5 (picture only) \\
\hline empirical formula & $\mathrm{C}_{38} \mathrm{H}_{42} \mathrm{~F}_{6} \mathrm{Fe}_{2} \mathrm{~N}_{14} \mathrm{O}_{7} \mathrm{~S}_{2}$ & $\mathrm{C}_{35} \mathrm{H}_{40} \mathrm{~F}_{9} \mathrm{Fe}_{2} \mathrm{~N}_{10} \mathrm{O}_{10} \mathrm{~S}_{3}+\mathrm{x}$ \\
\hline formula weight & 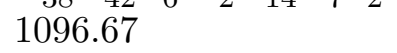 & 1139.65 \\
\hline $\mathrm{T}[\mathrm{K}]$ & $133(2)$ & $133(2)$ \\
\hline crystal size $\left[\mathrm{mm}^{3}\right]$ & $0.399 \times 0.208 \times 0.110$ & $0.440 \times 0.310 \times 0.130$ \\
\hline crystal system & triclinic & monoclinic \\
\hline space group & $P \overline{1}$ & $P 2 / \mathrm{n}$ \\
\hline $\mathrm{a}[\AA]$ & $13.0663(16)$ & $23.209(5)$ \\
\hline $\mathrm{b}[\AA]$ & $13.4599(15)$ & $17.555(4)$ \\
\hline$c[\AA]$ & $15.1620(19)$ & $28.512(6)$ \\
\hline$\alpha\left[^{\circ}\right]$ & $108.134(9)$ & 90 \\
\hline$\beta\left[^{\circ}\right]$ & $112.533(9)$ & $111.57(3)$ \\
\hline$\gamma\left[{ }^{\circ}\right]$ & $93.310(9)$ & 90 \\
\hline $\mathrm{V}\left[\AA^{3}\right]$ & $2292.6(5)$ & $10803(4)$ \\
\hline $\mathrm{Z}$ & 2 & 8 \\
\hline$\rho\left[\mathrm{g} / \mathrm{cm}^{3}\right]$ & 1.589 & 1.401 \\
\hline $\mathrm{F}(000)$ & 1124 & 4648 \\
\hline$\mu\left[\mathrm{mm}^{-1}\right]$ & 0.813 & 0.740 \\
\hline $\mathrm{T}_{\min } / \mathrm{T}_{\max }$ & $0.3419 / 0.8397$ & $-/-$ \\
\hline$\theta$-range $\left[^{\circ}\right]$ & $1.560-26.969$ & $1.391-25.712$ \\
\hline \multirow[t]{3}{*}{ hkl-range } & $-16 \leq \mathrm{h} \leq 16$ & $-27 \leq \mathrm{h} \leq 28$ \\
\hline & $-17 \leq \mathrm{k} \leq 17$ & $-21 \leq \mathrm{k} \leq 21$ \\
\hline & $-17 \leq 1 \leq 19$ & $-34 \leq 1 \leq 34$ \\
\hline measured refl. & 24941 & 101725 \\
\hline unique refl. $\left[\mathrm{R}_{\text {int }}\right]$ & $9815[0.1391]$ & $20444[0.1891]$ \\
\hline observed refl. $(\mathrm{I}>2 \sigma(\mathrm{I}))$ & 4399 & 11958 \\
\hline data / restraints / param. & $9815 / 0 / 627$ & $20444 / 0 / 1308$ \\
\hline goodness-of-fit ( $\left.\mathrm{F}^{2}\right)$ & 0.897 & 1.483 \\
\hline $\mathrm{R} 1, \mathrm{wR} 2(\mathrm{I}>2 \sigma(\mathrm{I}))$ & $0.0804,0.1737$ & $0.1709,0.4201$ \\
\hline R1, wR2 (all data) & $0.1761,0.2103$ & $0.2240,0.4509$ \\
\hline resid. el. dens. $\left[\mathrm{e} / \AA^{3}\right]$ & $-0.935 / 0.986$ & $-0.975 / 4.325$ \\
\hline
\end{tabular}


Table 10.5: Crystal data and refinement details for $5^{\text {Red }}$ and 6 (picture only).

\begin{tabular}{|c|c|c|}
\hline compound & $5^{\operatorname{Red}}$ & 6 (picture only) \\
\hline empirical formula & $\mathrm{C}_{43} \mathrm{H}_{42} \mathrm{~F}_{6} \mathrm{Fe}_{2} \mathrm{~N}_{10} \mathrm{O}_{8} \mathrm{~S}_{2}$ & $\mathrm{C}_{42} \mathrm{H}_{47} \mathrm{Fe}_{2} \mathrm{~N}_{10} \mathrm{O}_{12}+\mathrm{x}$ \\
\hline formula weight & 1116.68 & 3770.39 \\
\hline $\mathrm{T}[\mathrm{K}]$ & $133(2)$ & $133(2)$ \\
\hline crystal size $\left[\mathrm{mm}^{3}\right]$ & $0.324 \times 0.241 \times 0.100$ & $0.176 \times 0.165 \times 0.139$ \\
\hline crystal system & monoclinic & trigonal \\
\hline space group & $P 2_{1}$ & $R \overline{3}$ \\
\hline $\mathrm{a}[\AA]$ & $10.3381(3)$ & $29.140(4)$ \\
\hline $\mathrm{b}[\AA]$ & $15.1244(6)$ & $29.140(4)$ \\
\hline$c[\AA]$ & $15.2097(5)$ & $32.636(6)$ \\
\hline$\alpha\left[^{\circ}\right]$ & 90 & 90 \\
\hline$\beta\left[^{\circ}\right]$ & $100.368(3)$ & 90 \\
\hline$\gamma\left[{ }^{0}\right]$ & 90 & 120 \\
\hline $\mathrm{V}\left[\AA^{3}\right]$ & $2339.32(14)$ & $24000(8)$ \\
\hline Z & 2 & 6 \\
\hline$\rho\left[\mathrm{g} / \mathrm{cm}^{3}\right]$ & 1.585 & 1.565 \\
\hline $\mathrm{F}(000)$ & 1144 & 11808 \\
\hline$\mu\left[\mathrm{mm}^{-1}\right]$ & 0.798 & 0.799 \\
\hline $\mathrm{T}_{\min } / \mathrm{T}_{\max }$ & $0.7131 / 0.9322$ & $-/-$ \\
\hline$\theta$-range $\left[^{\circ}\right]$ & $1.361-26.734$ & $1.398-25.749$ \\
\hline \multirow[t]{3}{*}{ hkl-range } & $-13 \leq \mathrm{h} \leq 12$ & $-35 \leq \mathrm{h} \leq 35$ \\
\hline & $-19 \leq \mathrm{k} \leq 19$ & $-35 \leq \mathrm{k} \leq 35$ \\
\hline & $-19 \leq 1 \leq 19$ & $-39 \leq 1 \leq 39$ \\
\hline measured refl. & 23402 & 73522 \\
\hline unique refl. $\left[\mathrm{R}_{\text {int }}\right]$ & $9887[0.0521]$ & $10122[0.3426]$ \\
\hline observed refl. $(\mathrm{I}>2 \sigma(\mathrm{I}))$ & 8447 & 3087 \\
\hline data / restraints / param. & $9887 / 20 / 719$ & $10122 / 0 / 558$ \\
\hline goodness-of-fit $\left(\mathrm{F}^{2}\right)$ & 0.976 & 1.062 \\
\hline $\mathrm{R} 1, \mathrm{wR} 2(\mathrm{I}>2 \sigma(\mathrm{I}))$ & $0.0361,0.0652$ & $0.1672,0.3696$ \\
\hline R1, wR2 (all data) & $0.0474,0.0680$ & $0.3388,0.4766$ \\
\hline resid. el. dens. $\left[\mathrm{e} / \AA^{3}\right]$ & $-0.193 / 0.343$ & $-0.452 / 0.831$ \\
\hline
\end{tabular}


Table 10.6: Crystal data and refinement details for $\mathbf{7}$ and $\mathbf{8}$ (picture only).

\begin{tabular}{lll}
\hline compound & $\mathbf{7}$ & $\mathbf{8}$ (picture only) \\
\hline empirical formula & $\mathrm{C}_{26} \mathrm{H}_{40} \mathrm{Fe}_{2} \mathrm{~N}_{6} \mathrm{O}_{17.50}$ & $\mathrm{C}_{46} \mathrm{H}_{38} \mathrm{~K}_{2} \mathrm{Mn}_{4} \mathrm{~N}_{12} \mathrm{O}_{16}+\mathrm{x}$ \\
formula weight & 828.34 & 1312.85 \\
$\mathrm{~T}[\mathrm{~K}]$ & $133(2)$ & $133(2)$ \\
crystal size $\left[\mathrm{mm}^{3}\right]$ & $0.160 \times 0.110 \times 0.060$ & $0.184 \times 0.136 \times 0.097$ \\
crystal system & triclinic & triclinic \\
space group & $P \overline{1}$ & $P \overline{1}$ \\
$\mathrm{a}[\AA]$ & $10.413(2)$ & $12.527(3)$ \\
$\mathrm{b}[\AA]$ & $12.794(3)$ & $16.758(3)$ \\
$\mathrm{c}[\AA]$ & $14.982(3)$ & $16.960(3)$ \\
$\alpha\left[^{\circ}\right]$ & $87.18(3)$ & $109.19(3)$ \\
$\beta\left[^{\circ}\right]$ & $73.04(3)$ & $111.22(3)$ \\
$\gamma\left[^{\circ}\right]$ & $68.02(3)$ & $98.68(3)$ \\
$\mathrm{V}\left[\AA^{3}\right]$ & $1766.5(8)$ & $2983.6(13)$ \\
$\mathrm{Z}$ & 2 & 4 \\
$\rho\left[^{\mathrm{g}} / \mathrm{cm}^{3}\right]$ & 1.557 & 2.219 \\
$\mathrm{~F}(000)$ & 860 & 2048 \\
$\mu\left[\mathrm{mm}{ }^{-1}\right]$ & 0.905 & 1.225 \\
$\mathrm{~T}_{\text {min }} / \mathrm{T}_{\text {max }}$ & $0.7735 / 0.9341$ & $-/-$ \\
$\theta$-range [ $]$ & $1.424-26.755$ & $1.352-26.781$ \\
hkl-range & $-13 \leq \mathrm{h} \leq 13$ & $-15 \leq \mathrm{h} \leq 15$ \\
& $-16 \leq \mathrm{k} \leq 15$ & $-21 \leq \mathrm{k} \leq 21$ \\
& $-18 \leq 1 \leq 18$ & $-21 \leq 1 \leq 21$ \\
measured refl. & 18032 & 30910 \\
unique refl. $\left[\mathrm{R}_{\text {int }}\right]$ & $7476[0.1089]$ & $12654[0.1035]$ \\
observed refl. $(\mathrm{I}>2 \sigma(\mathrm{I}))$ & 4298 & 6904 \\
data / restraints / param. & $7476 / 27 / 526$ & $12654 / 1 / 720$ \\
goodness-of-fit $\left(\mathrm{F}^{2}\right)$ & 0.958 & 1.038 \\
$\mathrm{R} 1$, wR2 $(\mathrm{I}>2 \sigma(\mathrm{I}))$ & $0.0659,0.0908$ & $0.1136,0.2963$ \\
$\mathrm{R} 1$, wR2 (all data) & $0.1340,0.1064$ & $0.1799,0.3365$ \\
resid. el. dens. $\left[\mathrm{e} / \AA^{3}\right]$ & $-0.628 / 0.440$ & $-0.608 / 7.282$ \\
\hline & & \\
\hline
\end{tabular}


Table 10.7: Selected bond lengths (sorted) $[\AA]$ for $\mathbf{1 a}$.

\begin{tabular}{ll|ll}
\hline Atoms & Bond lengths & Atoms & Bond lengths \\
\hline Fe1-N9 & $1.901(5)$ & Fe2-N7 & $2.053(5)$ \\
Fe1-N15 & $1.938(6)$ & Fe1-N10 & $2.066(6)$ \\
Fe1-N1 & $1.948(6)$ & Fe2-N2 & $2.071(5)$ \\
Fe1-N11 & $1.955(6)$ & Fe2-N3 & $2.109(5)$ \\
Fe1-N13 & $1.996(6)$ & Fe2-N4 & $2.385(5)$ \\
Fe2-N5 & $2.052(5)$ & & \\
\hline
\end{tabular}

Table 10.8: Selected bond angles (sorted) $\left[^{\circ}\right]$ for $\mathbf{1 a}$.

\begin{tabular}{|c|c|c|c|}
\hline Atoms & Bond angles & Atoms & Bond angles \\
\hline N3-Fe2-N4 & $73.77(19)$ & C32-N13-Fe1 & $112.0(5)$ \\
\hline N7-Fe2-N4 & $76.37(19)$ & $\mathrm{C} 27-\mathrm{N} 11-\mathrm{Fe} 1$ & $113.0(5)$ \\
\hline N2-Fe2-N3 & $76.7(2)$ & N7-Fe2-N3 & $114.3(2)$ \\
\hline $\mathrm{N} 5-\mathrm{Fe} 2-\mathrm{N} 4$ & $76.94(19)$ & $\mathrm{C} 1-\mathrm{N} 1-\mathrm{Fe} 1$ & $115.9(4)$ \\
\hline N9-Fe1-N1 & $80.3(2)$ & $\mathrm{C} 3-\mathrm{N} 2-\mathrm{Fe} 2$ & $116.5(4)$ \\
\hline N13-Fe1-N10 & $81.2(2)$ & $\mathrm{N} 5-\mathrm{Fe} 2-\mathrm{N} 3$ & $116.9(2)$ \\
\hline N11-Fe1-N10 & $82.8(2)$ & $\mathrm{C} 11-\mathrm{N} 5-\mathrm{Fe} 2$ & $117.3(4)$ \\
\hline N9-Fe1-N10 & $82.9(2)$ & $\mathrm{C} 16-\mathrm{N} 7-\mathrm{Fe} 2$ & $117.3(4)$ \\
\hline N15-Fe1-N13 & $86.7(2)$ & $\mathrm{C} 4-\mathrm{N} 3-\mathrm{Fe} 2$ & $117.7(4)$ \\
\hline N9-Fe1-N11 & $88.8(2)$ & C20-N9-Fe1 & $118.8(4)$ \\
\hline N15-Fe1-N11 & $90.2(2)$ & C24-N9-Fe1 & $120.1(5)$ \\
\hline N1-Fe1-N11 & $93.8(2)$ & $\mathrm{C} 8-\mathrm{N} 3-\mathrm{Fe} 2$ & $122.4(4)$ \\
\hline N9-Fe1-N13 & $94.5(2)$ & $\mathrm{N} 5-\mathrm{Fe} 2-\mathrm{N} 2$ & $124.5(2)$ \\
\hline N15-Fe1-N10 & $97.4(2)$ & $\mathrm{N} 1-\mathrm{N} 2-\mathrm{Fe} 2$ & $135.5(4)$ \\
\hline N15-Fe1-N1 & $99.3(2)$ & $\mathrm{N} 2-\mathrm{N} 1-\mathrm{Fe} 1$ & $135.7(4)$ \\
\hline N1-Fe1-N13 & $103.0(2)$ & $\mathrm{C} 12-\mathrm{N} 5-\mathrm{Fe} 2$ & $136.0(4)$ \\
\hline $\mathrm{C} 15-\mathrm{N} 4-\mathrm{Fe} 2$ & $106.6(3)$ & $\mathrm{C} 17-\mathrm{N} 7-\mathrm{Fe} 2$ & $137.8(4)$ \\
\hline C10-N4-Fe2 & $107.1(4)$ & C28-N11-Fe1 & $140.2(5)$ \\
\hline C31-N10-Fe1 & $107.6(4)$ & C33-N13-Fe1 & $141.4(5)$ \\
\hline $\mathrm{C} 9-\mathrm{N} 4-\mathrm{Fe} 2$ & $107.7(4)$ & $\mathrm{N} 2-\mathrm{Fe} 2-\mathrm{N} 4$ & $149.5(2)$ \\
\hline C26-N10-Fe1 & $108.4(4)$ & N1-Fe1-N10 & $162.9(2)$ \\
\hline $\mathrm{C} 25-\mathrm{N} 10-\mathrm{Fe} 1$ & $108.7(4)$ & N11-Fe1-N13 & $163.2(2)$ \\
\hline $\mathrm{N} 7-\mathrm{Fe} 2-\mathrm{N} 2$ & $109.6(2)$ & C36-N15-Fe1 & $174.8(6)$ \\
\hline N5-Fe2-N7 & $111.0(2)$ & N9-Fe1-N15 & $178.8(2)$ \\
\hline
\end{tabular}


Table 10.9: Selected bond lengths (sorted) $[\AA]$ for $\mathbf{1 b}$.

\begin{tabular}{ll|ll}
\hline Atoms & Bond lengths & Atoms & Bond lengths \\
\hline Fe2-N5 & $2.050(3)$ & Fe2-N3 & $2.148(3)$ \\
Fe2-N7 & $2.083(3)$ & Fe1-N9 & $2.185(3)$ \\
Fe1-N11 & $2.094(3)$ & Fe1-N15 & $2.290(3)$ \\
Fe2-N2 & $2.100(3)$ & Fe2-N4 & $2.404(3)$ \\
Fe1-N1 & $2.109(3)$ & Fe1-N10 & $2.475(4)$ \\
Fe1-N13 & $2.109(3)$ & & \\
\hline
\end{tabular}

Table 10.10: Selected bond angles (sorted) $\left.{ }^{\circ}\right]$ for $\mathbf{1 b}$.

\begin{tabular}{ll|ll}
\hline Atoms & Bond angles & Atoms & Bond angles \\
\hline N3-Fe2-N4 & $72.52(13)$ & C4-N3-Fe2 & $116.5(3)$ \\
N1-Fe1-N9 & $74.99(13)$ & C20-N9-Fe1 & $116.6(3)$ \\
N7-Fe2-N4 & $75.50(11)$ & C11-N5-Fe2 & $118.2(3)$ \\
N5-Fe2-N4 & $75.78(12)$ & N7-Fe2-N3 & $118.95(12)$ \\
N2-Fe2-N3 & $75.97(13)$ & N7-Fe2-N2 & $119.08(11)$ \\
N11-Fe1-N15 & $82.32(11)$ & N5-Fe2-N2 & $119.43(12)$ \\
N1-Fe1-N15 & $85.69(12)$ & C32-N13-Fe1 & $120.4(2)$ \\
N13-Fe1-N15 & $91.89(11)$ & C24-N9-Fe1 & $122.1(3)$ \\
N13-Fe1-N9 & $94.98(11)$ & C8-N3-Fe2 & $123.0(3)$ \\
N1-Fe1-N13 & $99.48(12)$ & N11-Fe1-N13 & $129.08(14)$ \\
C15-N4-Fe2 & $105.0(2)$ & N11-Fe1-N1 & $130.08(13)$ \\
C10-N4-Fe2 & $106.3(2)$ & C28-N11-Fe1 & $131.8(3)$ \\
N11-Fe1-N9 & $107.13(12)$ & C33-N13-Fe1 & $133.0(2)$ \\
N5-Fe2-N7 & $108.45(12)$ & C12-N5-Fe2 & $134.8(3)$ \\
C9-N4-Fe2 & $108.9(3)$ & N2-N1-Fe1 & $135.4(2)$ \\
N5-Fe2-N3 & $111.98(12)$ & N1-N2-Fe2 & $136.4(2)$ \\
C3-N2-Fe2 & $115.4(3)$ & C17-N7-Fe2 & $138.3(2)$ \\
C16-N7-Fe2 & $115.7(2)$ & $\mathrm{C} 200-\mathrm{N} 15-\mathrm{Fe} 1$ & $148.1(3)$ \\
C27-N11-Fe1 & $116.3(3)$ & N2-Fe2-N4 & $148.39(12)$ \\
C1-N1-Fe1 & $116.5(3)$ & N9-Fe1-N15 & $160.30(13)$ \\
\hline
\end{tabular}


Table 10.11: Selected bond lengths (sorted) $[\AA]$ for $\mathbf{2 a}$.

\begin{tabular}{ll|ll}
\hline Atoms & Bond lengths & Atoms & Bond lengths \\
\hline Fe3-N23 & $1.895(4)$ & Fe2-N10 & $1.983(4)$ \\
Fe4-N27 & $1.895(4)$ & Fe4-N30 & $1.986(4)$ \\
Fe1-N3 & $1.897(4)$ & Fe1-N5 & $1.997(4)$ \\
Fe2-N7 & $1.906(4)$ & Fe1-N4 & $1.999(4)$ \\
Fe2-N12 & $1.925(4)$ & Fe2-N2 & $2.000(4)$ \\
Fe3-N31 & $1.932(4)$ & Fe2-N8 & $2.000(4)$ \\
Fe1-N11 & $1.938(4)$ & Fe3-N25 & $2.001(4)$ \\
Fe4-N32 & $1.944(4)$ & Fe1-N1 & $2.006(4)$ \\
Fe4-N29 & $1.964(4)$ & Fe3-N21 & $2.006(4)$ \\
Fe2-N9 & $1.966(4)$ & Fe3-N24 & $2.006(4)$ \\
Fe1-N6 & $1.970(4)$ & Fe4-N28 & $2.006(3)$ \\
Fe3-N26 & $1.972(4)$ & Fe4-N22 & $2.017(3)$ \\
\hline
\end{tabular}

Table 10.12: Selected bond angles (sorted) $\left[^{\circ}\right]$ for $\mathbf{2 a}$.

\begin{tabular}{llll}
\hline Atoms & Bond angles & Atoms & Bond angles \\
\hline N3-Fe1-N1 & $80.17(16)$ & C84-N28-Fe4 & $107.2(2)$ \\
N23-Fe3-N21 & $80.43(15)$ & C10-N4-Fe1 & $107.5(3)$ \\
N27-Fe4-N22 & $80.56(14)$ & C27-N8-Fe2 & $109.7(3)$ \\
N7-Fe2-N2 & $80.75(16)$ & C77-N28-Fe4 & $109.7(3)$ \\
N29-Fe4-N28 & $82.61(14)$ & C9-N4-Fe1 & $110.2(3)$ \\
N6-Fe1-N4 & $82.81(15)$ & C59-N24-Fe3 & $110.2(3)$ \\
N9-Fe2-N8 & $82.81(16)$ & C53-N22-Fe4 & $110.9(3)$ \\
N26-Fe3-N24 & $82.98(15)$ & C3-N2-Fe2 & $111.7(3)$ \\
N25-Fe3-N24 & $83.09(14)$ & C2-N1-Fe1 & $112.5(3)$ \\
N5-Fe1-N4 & $83.50(15)$ & C61-N25-Fe3 & $112.7(3)$ \\
N10-Fe2-N8 & $83.57(16)$ & C52-N21-Fe3 & $113.0(3)$ \\
N30-Fe4-N28 & $83.77(14)$ & C35-N10-Fe2 & $113.1(3)$ \\
N27-Fe4-N28 & $83.78(15)$ & C11-N5-Fe1 & $113.3(3)$ \\
N7-Fe2-N8 & $83.92(16)$ & C17-N6-Fe1 & $113.3(3)$ \\
N3-Fe1-N4 & $84.06(16)$ & C85-N30-Fe4 & $113.7(3)$ \\
N23-Fe3-N24 & $84.16(15)$ & C79-N29-Fe4 & $113.8(3)$ \\
N27-Fe4-N30 & $84.56(15)$ & C29-N9-Fe2 & $113.9(3)$ \\
N7-Fe2-N10 & $84.57(15)$ & C67-N26-Fe3 & $114.2(3)$ \\
N3-Fe1-N5 & $86.11(15)$ & C72-N27-Fe4 & $118.2(3)$ \\
N32-Fe4-N30 & $86.57(15)$ & C26-N7-Fe2 & $118.4(3)$ \\
N11-Fe1-N5 & $86.61(15)$ & C8-N3-Fe1 & $118.7(3)$ \\
N31-Fe3-N25 & $87.88(14)$ & C22-N7-Fe2 & $119.0(3)$ \\
N23-Fe3-N25 & $88.00(15)$ & C54-N23-Fe3 & $119.2(3)$ \\
N12-Fe2-N10 & $88.26(15)$ & C76-N27-Fe4 & $119.2(3)$ \\
N31-Fe3-N26 & $91.16(15)$ & C4-N3-Fe1 & $119.5(3)$ \\
N12-Fe2-N9 & $91.55(15)$ & C58-N23-Fe3 & $119.5(3)$ \\
N32-Fe4-N29 & $92.27(15)$ & C71-N26-Fe3 & $127.3(3)$ \\
& & &
\end{tabular}




\begin{tabular}{|c|c|c|c|}
\hline $\mathrm{N} 11-\mathrm{Fe} 1-\mathrm{N} 6$ & $92.76(15)$ & C33-N9-Fe2 & $128.2(3)$ \\
\hline $\mathrm{N} 23-\mathrm{Fe} 3-\mathrm{N} 26$ & $92.83(15)$ & $\mathrm{C} 83-\mathrm{N} 29-\mathrm{Fe} 4$ & $128.6(3)$ \\
\hline N3-Fe1-N6 & $94.14(15)$ & C39-N10-Fe2 & $128.7(3)$ \\
\hline N11-Fe1-N4 & $94.46(15)$ & $\mathrm{C} 89-\mathrm{N} 30-\mathrm{Fe} 4$ & $128.8(3)$ \\
\hline $\mathrm{N} 32-\mathrm{Fe} 4-\mathrm{N} 28$ & $94.93(14)$ & $\mathrm{C} 15-\mathrm{N} 5-\mathrm{Fe} 1$ & $129.1(3)$ \\
\hline $\mathrm{N} 12-\mathrm{Fe} 2-\mathrm{N} 8$ & $95.20(15)$ & $\mathrm{C} 21-\mathrm{N} 6-\mathrm{Fe} 1$ & 129.1(3) \\
\hline N31-Fe3-N24 & $95.32(15)$ & $\mathrm{C} 65-\mathrm{N} 25-\mathrm{Fe} 3$ & $129.3(3)$ \\
\hline N7-Fe2-N9 & $95.39(15)$ & $\mathrm{N} 1-\mathrm{N} 2-\mathrm{Fe} 2$ & $138.8(3)$ \\
\hline N26-Fe3-N21 & $95.93(15)$ & N22-N21-Fe3 & $139.7(3)$ \\
\hline N6-Fe1-N1 & $96.01(15)$ & $\mathrm{N} 21-\mathrm{N} 22-\mathrm{Fe} 4$ & $139.9(3)$ \\
\hline N27-Fe4-N29 & $96.27(15)$ & $\mathrm{N} 2-\mathrm{N} 1-\mathrm{Fe} 1$ & $140.0(3)$ \\
\hline $\mathrm{N} 10-\mathrm{Fe} 2-\mathrm{N} 2$ & $96.54(16)$ & $\mathrm{N} 4-\mathrm{Fe} 1-\mathrm{N} 1$ & $164.07(15)$ \\
\hline $\mathrm{N} 30-\mathrm{Fe} 4-\mathrm{N} 22$ & $96.61(14)$ & $\mathrm{N} 28-\mathrm{Fe} 4-\mathrm{N} 22$ & $164.22(15)$ \\
\hline N9-Fe2-N2 & $96.97(16)$ & $\mathrm{N} 21-\mathrm{Fe} 3-\mathrm{N} 24$ & $164.48(15)$ \\
\hline $\mathrm{N} 29-\mathrm{Fe} 4-\mathrm{N} 22$ & $97.13(14)$ & $\mathrm{N} 2-\mathrm{Fe} 2-\mathrm{N} 8$ & $164.59(15)$ \\
\hline N5-Fe1-N1 & $97.61(15)$ & N26-Fe3-N25 & $165.88(15)$ \\
\hline N25-Fe3-N21 & $98.10(15)$ & N29-Fe4-N30 & $166.18(14)$ \\
\hline $\mathrm{N} 31-\mathrm{Fe} 3-\mathrm{N} 21$ & $100.18(14)$ & N6-Fe1-N5 & $166.21(16)$ \\
\hline $\mathrm{N} 12-\mathrm{Fe} 2-\mathrm{N} 2$ & $100.21(15)$ & N9-Fe2-N10 & $166.30(16)$ \\
\hline $\mathrm{N} 32-\mathrm{Fe} 4-\mathrm{N} 22$ & $100.84(14)$ & N27-Fe4-N32 & $171.12(15)$ \\
\hline $\mathrm{N} 11-\mathrm{Fe} 1-\mathrm{N} 1$ & $101.47(15)$ & N3-Fe1-N11 & $172.69(16)$ \\
\hline $\mathrm{C} 16-\mathrm{N} 4-\mathrm{Fe} 1$ & $105.6(3)$ & $\mathrm{N} 7-\mathrm{Fe} 2-\mathrm{N} 12$ & $172.83(16)$ \\
\hline $\mathrm{C} 78-\mathrm{N} 28-\mathrm{Fe} 4$ & $105.8(2)$ & $\mathrm{C} 92-\mathrm{N} 32-\mathrm{Fe} 4$ & $173.2(4)$ \\
\hline $\mathrm{C} 60-\mathrm{N} 24-\mathrm{Fe} 3$ & $106.4(3)$ & $\mathrm{C} 40-\mathrm{N} 11-\mathrm{Fe} 1$ & $173.5(4)$ \\
\hline C28-N8-Fe2 & $106.6(3)$ & N23-Fe3-N31 & $175.88(15)$ \\
\hline $\mathrm{C} 34-\mathrm{N} 8-\mathrm{Fe} 2$ & $106.7(3)$ & $\mathrm{C} 42-\mathrm{N} 12-\mathrm{Fe} 2$ & $176.4(4)$ \\
\hline $\mathrm{C} 66-\mathrm{N} 24-\mathrm{Fe} 3$ & $106.8(2)$ & C90-N31-Fe3 & $176.9(4)$ \\
\hline
\end{tabular}

Table 10.13: Selected bond lengths (sorted) $[\AA]$ for $\mathbf{2 b}$.

\begin{tabular}{ll|ll}
\hline Atoms & Bond lengths & Atoms & Bond lengths \\
\hline Fe2-N7 & $1.899(3)$ & Fe1-N5 & $1.988(3)$ \\
Fe1-N3 & $1.900(3)$ & Fe2-N10 & $1.995(3)$ \\
Fe1-N11 & $1.942(3)$ & Fe2-N8 & $2.000(3)$ \\
Fe2-N12 & $1.954(3)$ & Fe1-N1 & $2.003(3)$ \\
Fe1-N6 & $1.969(3)$ & Fe1-N4 & $2.009(3)$ \\
Fe2-N9 & $1.975(3)$ & Fe2-N2 & $2.024(3)$ \\
\hline
\end{tabular}


Table 10.14: Selected bond angles (sorted) $\left.{ }^{\circ}\right]$ for $\mathbf{2 b}$.

\begin{tabular}{|c|c|c|c|}
\hline Atoms & Bond angles & Atoms & Bond angles \\
\hline N7-Fe2-N2 & $80.42(12)$ & C34-N8-Fe2 & $107.7(2)$ \\
\hline N3-Fe1-N1 & $81.01(12)$ & C9-N4-Fe1 & $109.3(2)$ \\
\hline N9-Fe2-N8 & $82.24(12)$ & $\mathrm{C} 27-\mathrm{N} 8-\mathrm{Fe} 2$ & $110.5(2)$ \\
\hline $\mathrm{N} 5-\mathrm{Fe} 1-\mathrm{N} 4$ & $83.58(12)$ & C3-N1-Fe1 & $111.6(2)$ \\
\hline N6-Fe1-N4 & $83.65(12)$ & $\mathrm{C} 1-\mathrm{N} 2-\mathrm{Fe} 2$ & $111.8(2)$ \\
\hline N10-Fe2-N8 & $83.92(12)$ & $\mathrm{C} 35-\mathrm{N} 10-\mathrm{Fe} 2$ & $112.8(2)$ \\
\hline N7-Fe2-N8 & $84.09(13)$ & $\mathrm{C} 11-\mathrm{N} 5-\mathrm{Fe} 1$ & $112.9(3)$ \\
\hline N12-Fe2-N10 & $84.54(13)$ & C17-N6-Fe1 & $113.7(2)$ \\
\hline $\mathrm{N} 3-\mathrm{Fe} 1-\mathrm{N} 4$ & $84.63(12)$ & C29-N9-Fe2 & $114.0(2)$ \\
\hline N11-Fe1-N5 & $86.28(12)$ & C8-N3-Fe1 & $118.8(3)$ \\
\hline N7-Fe2-N10 & $87.82(13)$ & $\mathrm{C} 4-\mathrm{N} 3-\mathrm{Fe} 1$ & $118.9(2)$ \\
\hline N3-Fe1-N5 & $88.84(12)$ & $\mathrm{C} 26-\mathrm{N} 7-\mathrm{Fe} 2$ & $119.2(3)$ \\
\hline N11-Fe1-N6 & $90.25(12)$ & $\mathrm{C} 22-\mathrm{N} 7-\mathrm{Fe} 2$ & $119.6(2)$ \\
\hline N11-Fe1-N4 & $93.02(12)$ & C21-N6-Fe1 & $127.6(3)$ \\
\hline N12-Fe2-N9 & $93.40(13)$ & C33-N9-Fe2 & $127.7(2)$ \\
\hline $\mathrm{N} 7-\mathrm{Fe} 2-\mathrm{N} 9$ & $93.81(13)$ & $\mathrm{C} 15-\mathrm{N} 5-\mathrm{Fe} 1$ & $127.9(3)$ \\
\hline $\mathrm{N} 3-\mathrm{Fe} 1-\mathrm{N} 6$ & $94.10(12)$ & C39-N10-Fe2 & $128.9(3)$ \\
\hline $\mathrm{N} 12-\mathrm{Fe} 2-\mathrm{N} 8$ & $94.27(13)$ & $\mathrm{N} 2-\mathrm{N} 1-\mathrm{Fe} 1$ & $139.0(2)$ \\
\hline N9-Fe2-N2 & $95.80(12)$ & $\mathrm{N} 1-\mathrm{N} 2-\mathrm{Fe} 2$ & $140.2(2)$ \\
\hline N6-Fe1-N1 & $96.02(12)$ & $\mathrm{N} 8-\mathrm{Fe} 2-\mathrm{N} 2$ & $164.23(13)$ \\
\hline N5-Fe1-N1 & $97.38(12)$ & N1-Fe1-N4 & $165.57(12)$ \\
\hline $\mathrm{N} 10-\mathrm{Fe} 2-\mathrm{N} 2$ & $98.36(12)$ & N9-Fe2-N10 & $165.81(12)$ \\
\hline N11-Fe1-N1 & $101.41(12)$ & N6-Fe1-N5 & $166.57(13)$ \\
\hline $\mathrm{N} 12-\mathrm{Fe} 2-\mathrm{N} 2$ & $101.48(12)$ & $\mathrm{C} 42-\mathrm{N} 12-\mathrm{Fe} 2$ & $169.5(3)$ \\
\hline C16-N4-Fe1 & $105.7(2)$ & N7-Fe2-N12 & $172.32(12)$ \\
\hline C28-N8-Fe2 & $106.9(2)$ & $\mathrm{C} 40-\mathrm{N} 11-\mathrm{Fe} 1$ & $174.1(3)$ \\
\hline C10-N4-Fe1 & $107.4(2)$ & N3-Fe1-N11 & $174.79(12)$ \\
\hline
\end{tabular}

Table 10.15: Selected bond lengths (sorted) $[\AA]$ for 3 .

\begin{tabular}{ll|ll}
\hline Atoms & Bond lengths & Atoms & Bond lengths \\
\hline Fe1-N5 & $2.009(2)$ & Fe2-N1 & $2.125(3)$ \\
Fe1-O1 & $2.009(2)$ & Fe2-N13 & $2.158(3)$ \\
Fe2-O2 & $2.010(2)$ & Fe1-N3 & $2.217(3)$ \\
Fe1-N2 & $2.013(3)$ & Fe2-N9 & $2.373(3)$ \\
Fe1-N7 & $2.017(3)$ & Fe1-N4 & $2.421(3)$ \\
Fe2-O1 & $2.038(2)$ & Fe2-N10 & $2.469(3)$ \\
Fe2-N11 & $2.059(2)$ & & \\
\hline
\end{tabular}


Table 10.16: Selected bond angles (sorted) $\left.{ }^{\circ}\right]$ for $\mathbf{3}$.

\begin{tabular}{ll|ll}
\hline Atoms & Bond angles & Atoms & Bond angles \\
\hline N1-Fe2-N9 & $67.98(9)$ & C16-N7-Fe1 & $118.77(19)$ \\
N3-Fe1-N4 & $70.59(9)$ & C32-N11-Fe2 & $118.9(2)$ \\
N2-Fe1-N3 & $72.19(10)$ & C27-N13-Fe2 & $119.9(2)$ \\
N7-Fe1-N4 & $73.67(9)$ & Fe2-O1-H1 & $121(3)$ \\
O1-Fe2-N1 & $74.40(9)$ & C11-N5-Fe1 & $122.2(2)$ \\
N5-Fe1-N4 & $74.56(10)$ & C8-N3-Fe1 & $122.6(2)$ \\
O1-Fe1-N2 & $77.02(10)$ & C24-N9-Fe2 & $122.7(2)$ \\
O1-Fe2-N13 & $80.80(9)$ & Fe2-O2-H2B & $123(5)$ \\
N11-Fe2-N9 & $82.52(10)$ & N2-Fe1-N7 & $123.87(10)$ \\
O2-Fe2-N9 & $83.10(10)$ & N2-N1-Fe2 & $124.00(19)$ \\
N11-Fe2-N1 & $87.23(10)$ & C3-N2-Fe1 & $124.9(2)$ \\
O1-Fe1-N7 & $88.65(10)$ & N1-N2-Fe1 & $125.97(19)$ \\
N11-Fe2-N13 & $89.41(10)$ & Fe2-O2-H3B & $126(3)$ \\
N5-Fe1-O1 & $89.57(10)$ & N5-Fe1-N7 & $126.16(11)$ \\
O2-Fe2-N13 & $92.13(11)$ & C1-N1-Fe2 & $126.2(2)$ \\
O2-Fe2-O1 & $96.37(10)$ & C12-N5-Fe1 & $130.7(2)$ \\
O2-Fe2-N1 & $99.80(10)$ & C17-N7-Fe1 & $132.1(2)$ \\
Fe1-O1-H1 & $102(3)$ & C33-N11-Fe2 & $134.1(2)$ \\
N7-Fe1-N3 & $102.86(10)$ & C28-N13-Fe2 & $134.1(2)$ \\
O1-Fe2-N11 & $103.31(10)$ & Fe1-O1-Fe2 & $136.26(13)$ \\
C15-N4-Fe1 & $104.68(17)$ & N13-Fe2-N9 & $137.54(9)$ \\
N5-Fe1-N3 & $106.00(10)$ & O1-Fe1-N4 & $140.83(10)$ \\
N5-Fe1-N2 & $107.94(11)$ & O1-Fe2-N9 & $141.63(9)$ \\
C10-N4-Fe1 & $108.40(18)$ & N2-Fe1-N4 & $141.73(9)$ \\
C9-N4-Fe1 & $109.63(18)$ & O1-Fe1-N3 & $148.49(10)$ \\
C4-N3-Fe1 & $117.8(2)$ & N1-Fe2-N13 & $153.45(10)$ \\
C20-N9-Fe2 & $118.23(19)$ & O2-Fe2-N11 & $160.26(10)$ \\
\hline
\end{tabular}

Table 10.17: Selected bond lengths (sorted) $[\AA]$ for 4.

\begin{tabular}{ll|ll}
\hline Atoms & Bond lengths & Atoms & Bond lengths \\
\hline Fe2-N22B & $1.70(3)$ & Fe1-N11 & $2.101(3)$ \\
Fe1-N21A & $1.775(4)$ & Fe2-N5 & $2.101(3)$ \\
Fe2-N22A & $1.788(6)$ & Fe2-N7 & $2.107(3)$ \\
Fe1-N21B & $1.85(13)$ & Fe2-N3 & $2.122(3)$ \\
Fe1-N1 & $2.071(3)$ & Fe1-N9 & $2.130(3)$ \\
Fe2-N2 & $2.079(3)$ & Fe2-N4 & $2.351(3)$ \\
Fe1-N13 & $2.091(3)$ & Fe1-N10 & $2.357(3)$ \\
\hline
\end{tabular}


Table 10.18: Selected bond angles (sorted) $\left[^{\circ}\right]$ for 4 .

\begin{tabular}{|c|c|c|c|}
\hline Atoms & Bond angles & Atoms & Bond angles \\
\hline N13-Fe1-N10 & $73.89(10)$ & N21B-Fe1-N10 & $108(4)$ \\
\hline $\mathrm{N} 7-\mathrm{Fe} 2-\mathrm{N} 4$ & $74.55(10)$ & C26-N10-Fe1 & $108.70(19)$ \\
\hline N21B-Fe1-N11 & $75(4)$ & $\mathrm{C} 9-\mathrm{N} 4-\mathrm{Fe} 2$ & $109.47(19)$ \\
\hline $\mathrm{N} 5-\mathrm{Fe} 2-\mathrm{N} 4$ & $75.22(10)$ & $\mathrm{N} 22 \mathrm{~B}-\mathrm{Fe} 2-\mathrm{N} 2$ & $109.5(15)$ \\
\hline N9-Fe1-N10 & $75.44(10)$ & N21B-Fe1-N13 & $112(3)$ \\
\hline N11-Fe1-N10 & $75.55(10)$ & $\mathrm{C} 1-\mathrm{N} 1-\mathrm{Fe} 1$ & $115.5(2)$ \\
\hline $\mathrm{N} 3-\mathrm{Fe} 2-\mathrm{N} 4$ & $75.94(10)$ & $\mathrm{C} 20-\mathrm{N} 9-\mathrm{Fe} 1$ & $115.6(2)$ \\
\hline N1-Fe1-N9 & $75.97(10)$ & C32-N13-Fe1 & $115.9(2)$ \\
\hline $\mathrm{N} 2-\mathrm{Fe} 2-\mathrm{N} 3$ & $76.55(10)$ & $\mathrm{C} 16-\mathrm{N} 7-\mathrm{Fe} 2$ & $116.0(2)$ \\
\hline N11-Fe1-N9 & $81.30(11)$ & $\mathrm{C} 3-\mathrm{N} 2-\mathrm{Fe} 2$ & $116.2(2)$ \\
\hline $\mathrm{N} 7-\mathrm{Fe} 2-\mathrm{N} 3$ & $86.22(11)$ & $\mathrm{C} 11-\mathrm{N} 5-\mathrm{Fe} 2$ & $116.9(2)$ \\
\hline N5-Fe2-N3 & $87.58(11)$ & $\mathrm{C} 4-\mathrm{N} 3-\mathrm{Fe} 2$ & $117.4(2)$ \\
\hline N22B-Fe2-N5 & $87.6(12)$ & C27-N11-Fe1 & $117.5(2)$ \\
\hline $\mathrm{N} 22 \mathrm{~A}-\mathrm{Fe} 2-\mathrm{N} 7$ & $87.9(3)$ & $\mathrm{C} 24-\mathrm{N} 9-\mathrm{Fe} 1$ & $120.5(2)$ \\
\hline N21A-Fe1-N11 & $88.56(16)$ & $\mathrm{C} 8-\mathrm{N} 3-\mathrm{Fe} 2$ & $122.0(2)$ \\
\hline N13-Fe1-N9 & $94.27(11)$ & $\mathrm{O} 1 \mathrm{~B}-\mathrm{N} 21 \mathrm{~B}-\mathrm{Fe} 1$ & $125(10)$ \\
\hline N22B-Fe2-N7 & $95.4(14)$ & $\mathrm{N} 1-\mathrm{N} 2-\mathrm{Fe} 2$ & $135.2(2)$ \\
\hline N21A-Fe1-N13 & $97.03(17)$ & C28-N11-Fe1 & $135.2(2)$ \\
\hline $\mathrm{N} 22 \mathrm{~A}-\mathrm{Fe} 2-\mathrm{N} 5$ & $97.3(2)$ & C33-N13-Fe1 & $135.9(2)$ \\
\hline $\mathrm{N} 22 \mathrm{~B}-\mathrm{Fe} 2-\mathrm{N} 4$ & $97.8(15)$ & $\mathrm{C} 12-\mathrm{N} 5-\mathrm{Fe} 2$ & $136.3(2)$ \\
\hline N21B-Fe1-N1 & $99(4)$ & $\mathrm{N} 2-\mathrm{N} 1-\mathrm{Fe} 1$ & $136.4(2)$ \\
\hline $\mathrm{N} 2-\mathrm{Fe} 2-\mathrm{N} 5$ & $100.47(11)$ & $\mathrm{C} 17-\mathrm{N} 7-\mathrm{Fe} 2$ & $137.0(2)$ \\
\hline N21A-Fe1-N1 & $101.74(16)$ & $\mathrm{O} 1 \mathrm{~A}-\mathrm{N} 21 \mathrm{~A}-\mathrm{Fe} 1$ & $147.0(5)$ \\
\hline $\mathrm{N} 22 \mathrm{~A}-\mathrm{Fe} 2-\mathrm{N} 4$ & $102.0(3)$ & N13-Fe1-N11 & $149.25(11)$ \\
\hline N1-Fe1-N11 & $103.49(11)$ & N5-Fe2-N7 & $149.74(11)$ \\
\hline N1-Fe1-N13 & $104.88(11)$ & N1-Fe1-N10 & $151.19(10)$ \\
\hline $\mathrm{C} 15-\mathrm{N} 4-\mathrm{Fe} 2$ & 105.37(18) & $\mathrm{O} 2 \mathrm{~A}-\mathrm{N} 22 \mathrm{~A}-\mathrm{Fe} 2$ & $151.6(10)$ \\
\hline $\mathrm{N} 22 \mathrm{~A}-\mathrm{Fe} 2-\mathrm{N} 2$ & $105.7(3)$ & $\mathrm{N} 2-\mathrm{Fe} 2-\mathrm{N} 4$ & $152.30(10)$ \\
\hline C31-N10-Fe1 & $106.26(19)$ & N21B-Fe1-N9 & $154(3)$ \\
\hline $\mathrm{N} 2-\mathrm{Fe} 2-\mathrm{N} 7$ & $106.78(11)$ & $\mathrm{O} 2 \mathrm{~B}-\mathrm{N} 22 \mathrm{~B}-\mathrm{Fe} 2$ & $165(4)$ \\
\hline $\mathrm{N} 21 \mathrm{~A}-\mathrm{Fe} 1-\mathrm{N} 10$ & $107.00(16)$ & N21A-Fe1-N9 & $168.67(16)$ \\
\hline $\mathrm{C} 25-\mathrm{N} 10-\mathrm{Fe} 1$ & $107.36(19)$ & $\mathrm{N} 22 \mathrm{~B}-\mathrm{Fe} 2-\mathrm{N} 3$ & $172.9(12)$ \\
\hline $\mathrm{C} 10-\mathrm{N} 4-\mathrm{Fe} 2$ & $107.51(19)$ & $\mathrm{N} 22 \mathrm{~A}-\mathrm{Fe} 2-\mathrm{N} 3$ & $174.1(3)$ \\
\hline
\end{tabular}

Table 10.19: Selected bond lengths (sorted) $[\AA]$ for $\mathbf{4}^{\text {Red }}$.

\begin{tabular}{ll|ll}
\hline Atoms & Bond lengths & Atoms & Bond lengths \\
\hline Fe1-O1 & $2.031(5)$ & Fe1-N11 & $2.174(5)$ \\
Fe2-N2 & $2.064(6)$ & Fe2-O1 & $2.176(4)$ \\
Fe1-N1 & $2.076(6)$ & Fe1-N9 & $2.268(6)$ \\
Fe2-N5 & $2.119(5)$ & Fe2-N3 & $2.340(6)$ \\
Fe2-N7 & $2.130(6)$ & Fe2-N3 & $2.341(7)$ \\
Fe1-N13 & $2.132(5)$ & Fe1-N10 & $2.463(5)$ \\
\hline
\end{tabular}


Table 10.20: Selected bond angles $\left[^{\circ}\right.$ for $4^{\text {Red }}$.

\begin{tabular}{ll|ll}
\hline Atoms & Bond angles & Atoms & Bond angles \\
\hline O1-Fe1-N1 & $81.2(2)$ & N2-N1-Fe1 & $127.2(5)$ \\
O1-Fe1-N13 & $93.1(2)$ & C1-N1-Fe1 & $124.8(4)$ \\
N1-Fe1-N13 & $121.8(2)$ & N1-N2-Fe2 & $124.2(5)$ \\
O1-Fe1-N11 & $101.3(2)$ & C3-N2-Fe2 & $126.2(5)$ \\
N1-Fe1-N11 & $94.6(2)$ & C8-N3-Fe2 & $124.4(5)$ \\
N13-Fe1-N11 & $142.7(2)$ & C4-N3-Fe2 & $117.0(4)$ \\
O1-Fe1-N9 & $152.1(2)$ & C11-N5-Fe2 & $122.5(4)$ \\
N1-Fe1-N9 & $70.9(2)$ & $\mathrm{C} 12-\mathrm{N} 5-\mathrm{Fe} 2$ & $131.8(5)$ \\
N13-Fe1-N9 & $100.4(2)$ & $\mathrm{C} 16-\mathrm{N} 7-\mathrm{Fe} 2$ & $119.6(4)$ \\
N11-Fe1-N9 & $82.6(2)$ & $\mathrm{C} 17-\mathrm{N} 7-\mathrm{Fe} 2$ & $133.5(5)$ \\
N2-Fe2-N5 & $116.1(2)$ & $\mathrm{C} 20-\mathrm{N} 9-\mathrm{Fe} 1$ & $119.1(5)$ \\
N2-Fe2-N7 & $128.9(2)$ & $\mathrm{C} 24-\mathrm{N} 9-\mathrm{Fe} 1$ & $120.8(5)$ \\
N5-Fe2-N7 & $114.6(2)$ & $\mathrm{C} 27-\mathrm{N} 11-\mathrm{Fe} 1$ & $117.9(4)$ \\
N2-Fe2-O1 & $80.5(2)$ & $\mathrm{C} 28-\mathrm{N} 11-\mathrm{Fe} 1$ & $133.6(5)$ \\
N5-Fe2-O1 & $87.44(19)$ & $\mathrm{C} 32-\mathrm{N} 13-\mathrm{Fe} 1$ & $119.5(4)$ \\
N7-Fe2-O1 & $95.5(2)$ & $\mathrm{C} 33-\mathrm{N} 13-\mathrm{Fe} 1$ & $133.3(5)$ \\
N2-Fe2-N3 & $69.8(2)$ & $\mathrm{C} 70-\mathrm{O} 1-\mathrm{Fe} 1$ & $118.1(4)$ \\
N5-Fe2-N3 & $107.8(2)$ & $\mathrm{C} 70-\mathrm{O} 1-\mathrm{Fe} 2$ & $115.6(4)$ \\
N7-Fe2-N3 & $100.8(2)$ & $\mathrm{Fe} 1-\mathrm{O} 1-\mathrm{Fe} 2$ & $126.3(2)$ \\
$\mathrm{O} 1-\mathrm{Fe} 2-\mathrm{N} 3$ & $150.2(2)$ & & \\
\hline
\end{tabular}

Table 10.21: Selected bond lengths (sorted) $[\AA]$ for $\mathbf{5}^{\text {Red }}$.

\begin{tabular}{ll|ll}
\hline Atoms & Bond lengths & Atoms & Bond lengths \\
\hline Fe01-O2 & $1.993(3)$ & Fe02-N6 & $2.172(3)$ \\
Fe02-O1 & $2.003(3)$ & Fe02-N5 & $2.205(3)$ \\
Fe02-N3 & $2.145(3)$ & Fe01-N10 & $2.211(3)$ \\
Fe02-N2 & $2.165(3)$ & Fe01-N9 & $2.211(3)$ \\
Fe01-N1 & $2.166(3)$ & Fe01-N8 & $2.292(3)$ \\
Fe01-N7 & $2.170(3)$ & Fe02-N4 & $2.298(3)$ \\
\hline
\end{tabular}


Table 10.22: Selected bond angles (sorted) [ $\left.{ }^{\circ}\right]$ for $\mathbf{5}^{\mathbf{R e d}}$.

\begin{tabular}{llll}
\hline Atoms & Bond angles & Atoms & Bond angles \\
\hline N10-Fe01-N8 & $73.60(11)$ & O2-Fe01-N8 & $112.79(12)$ \\
N1-Fe01-N7 & $74.41(13)$ & O1-Fe02-N4 & $113.38(13)$ \\
N3-Fe02-N2 & $74.91(13)$ & C29-N9-Fe01 & $114.3(3)$ \\
N7-Fe01-N8 & $75.20(12)$ & C3-N2-Fe02 & $114.9(3)$ \\
N5-Fe02-N4 & $75.27(13)$ & C35-N10-Fe01 & $115.1(3)$ \\
N6-Fe02-N4 & $75.35(13)$ & C11-N5-Fe02 & $115.7(3)$ \\
N3-Fe02-N4 & $75.81(13)$ & C1-N1-Fe01 & $116.1(3)$ \\
N9-Fe01-N8 & $76.84(12)$ & C17-N6-Fe02 & $116.3(3)$ \\
N7-Fe01-N9 & $81.01(12)$ & Fe02-O1-H1 & $117(3)$ \\
N3-Fe02-N5 & $82.27(13)$ & C4-N3-Fe02 & $117.6(3)$ \\
O1-Fe02-N5 & $88.64(13)$ & C22-N7-Fe01 & $118.2(3)$ \\
N3-Fe02-N6 & $89.31(13)$ & C26-N7-Fe01 & $120.0(3)$ \\
N7-Fe01-N10 & $90.44(12)$ & C8-N3-Fe02 & $120.2(3)$ \\
O2-Fe01-N10 & $92.61(12)$ & Fe01-O2-H1 & $123(4)$ \\
N1-Fe01-N9 & $94.99(13)$ & C21-N6-Fe02 & $125.1(3)$ \\
O1-Fe02-N2 & $96.68(13)$ & C33-N9-Fe01 & $125.4(3)$ \\
O2-Fe01-N1 & $97.57(12)$ & C15-N5-Fe02 & $125.7(3)$ \\
N2-Fe02-N6 & $97.57(13)$ & C39-N10-Fe01 & $125.8(3)$ \\
O2-Fe01-N9 & $99.74(12)$ & C41-O2-Fe01 & $128.4(3)$ \\
C34-N8-Fe01 & $104.0(2)$ & C40-O1-Fe02 & $129.9(3)$ \\
O1-Fe02-N6 & $104.24(13)$ & N2-N1-Fe01 & $135.7(3)$ \\
C16-N4-Fe02 & $104.5(2)$ & N1-N2-Fe02 & $137.1(3)$ \\
C10-N4-Fe02 & $104.7(2)$ & N1-Fe01-N8 & $149.41(13)$ \\
C28-N8-Fe01 & $105.1(2)$ & N2-Fe02-N4 & $149.94(12)$ \\
N2-Fe02-N5 & $107.20(13)$ & N10-Fe01-N9 & $150.42(12)$ \\
N1-Fe01-N10 & $109.99(13)$ & N6-Fe02-N5 & $150.59(13)$ \\
C9-N4-Fe02 & $111.1(3)$ & O1-Fe02-N3 & $165.03(14)$ \\
C27-N8-Fe01 & $111.1(3)$ & O2-Fe01-N7 & $171.98(13)$ \\
\hline
\end{tabular}

Table 10.23: Selected bond lengths (sorted) $[\AA]$ for 7 .

\begin{tabular}{ll|ll}
\hline Atoms & Bond lengths & Atoms & Bond lengths \\
\hline Fe2-O4 & $1.986(3)$ & Fe2-N2 & $2.089(4)$ \\
Fe1-O7 & $1.993(3)$ & Fe2-O2 & $2.106(4)$ \\
Fe2-O11 & $1.997(3)$ & Fe1-O5 & $2.126(4)$ \\
Fe1-O11 & $2.004(3)$ & Fe2-N3 & $2.308(4)$ \\
Fe2-O10 & $2.012(4)$ & Fe1-N5 & $2.315(4)$ \\
Fe1-O9 & $2.015(4)$ & Fe1-N6 & $2.344(4)$ \\
Fe1-N1 & $2.089(4)$ & Fe2-N4 & $2.385(4)$ \\
\hline
\end{tabular}


Table 10.24: Selected bond angles (sorted) $\left[{ }^{\circ}\right]$ for $\mathbf{7}$.

\begin{tabular}{|c|c|c|c|}
\hline Atoms & Bond angles & Atoms & Bond angles \\
\hline N5-Fe1-N6 & $68.98(14)$ & C12-N4-Fe2 & $104.2(3)$ \\
\hline N1-Fe1-N5 & $68.99(15)$ & C20-N6-Fe1 & $105.7(3)$ \\
\hline $\mathrm{N} 3-\mathrm{Fe} 2-\mathrm{N} 4$ & $69.29(15)$ & C10-N4-Fe2 & $106.0(3)$ \\
\hline $\mathrm{N} 2-\mathrm{Fe} 2-\mathrm{N} 3$ & $69.48(15)$ & C9-N4-Fe2 & 111.1(3) \\
\hline $\mathrm{O} 2-\mathrm{Fe} 2-\mathrm{N} 4$ & $71.50(13)$ & C19-N6-Fe1 & $113.8(3)$ \\
\hline $\mathrm{O} 5-\mathrm{Fe} 1-\mathrm{N} 6$ & $71.79(14)$ & $\mathrm{C} 4-\mathrm{N} 3-\mathrm{Fe} 2$ & $118.2(3)$ \\
\hline $\mathrm{O} 11-\mathrm{Fe} 2-\mathrm{N} 2$ & $74.42(15)$ & $\mathrm{C} 14-\mathrm{N} 5-\mathrm{Fe} 1$ & $118.2(3)$ \\
\hline O11-Fe1-N1 & $74.86(15)$ & C23-O7-Fe1 & $121.2(3)$ \\
\hline $\mathrm{O} 11-\mathrm{Fe} 2-\mathrm{O} 2$ & $75.30(13)$ & $\mathrm{C} 8-\mathrm{N} 3-\mathrm{Fe} 2$ & $121.3(4)$ \\
\hline $\mathrm{O} 11-\mathrm{Fe} 1-\mathrm{O} 5$ & $75.54(13)$ & $\mathrm{C} 21-\mathrm{O} 5-\mathrm{Fe} 1$ & $122.5(3)$ \\
\hline $\mathrm{O} 4-\mathrm{Fe} 2-\mathrm{N} 4$ & $77.61(13)$ & C18-N5-Fe1 & $122.7(3)$ \\
\hline $\mathrm{O} 7-\mathrm{Fe} 1-\mathrm{N} 6$ & $79.48(13)$ & $\mathrm{C} 11-\mathrm{O} 2-\mathrm{Fe} 2$ & $123.1(3)$ \\
\hline $\mathrm{O} 4-\mathrm{Fe} 2-\mathrm{N} 3$ & $83.30(14)$ & $\mathrm{C} 13-\mathrm{O} 4-\mathrm{Fe} 2$ & $123.5(3)$ \\
\hline $\mathrm{O} 10-\mathrm{Fe} 2-\mathrm{N} 3$ & $85.70(14)$ & N2-N1-Fe1 & $124.8(3)$ \\
\hline $\mathrm{O} 7-\mathrm{Fe} 1-\mathrm{N} 5$ & $86.28(15)$ & $\mathrm{C} 2-\mathrm{N} 2-\mathrm{Fe} 2$ & $125.7(3)$ \\
\hline $\mathrm{O} 10-\mathrm{Fe} 2-\mathrm{N} 4$ & $86.68(14)$ & $\mathrm{N} 1-\mathrm{N} 2-\mathrm{Fe} 2$ & $126.0(3)$ \\
\hline O7-Fe1-O5 & $88.07(14)$ & C3-N1-Fe1 & $126.6(3)$ \\
\hline O9-Fe1-N5 & $88.60(15)$ & N1-Fe1-N6 & $137.54(16)$ \\
\hline $\mathrm{O} 10-\mathrm{Fe} 2-\mathrm{O} 2$ & $89.75(15)$ & $\mathrm{N} 2-\mathrm{Fe} 2-\mathrm{N} 4$ & $138.00(14)$ \\
\hline $\mathrm{O} 4-\mathrm{Fe} 2-\mathrm{N} 2$ & $90.05(15)$ & $\mathrm{Fe} 2-\mathrm{O} 11-\mathrm{Fe} 1$ & $139.65(19)$ \\
\hline O9-Fe1-O5 & $90.62(15)$ & $\mathrm{O} 2-\mathrm{Fe} 2-\mathrm{N} 3$ & $140.71(15)$ \\
\hline O11-Fe1-O9 & $90.67(15)$ & $\mathrm{O} 5-\mathrm{Fe} 1-\mathrm{N} 5$ & $140.75(13)$ \\
\hline $\mathrm{O} 4-\mathrm{Fe} 2-\mathrm{O} 2$ & $90.77(14)$ & O11-Fe1-N5 & $143.71(13)$ \\
\hline O9-Fe1-N6 & $90.89(13)$ & $\mathrm{O} 11-\mathrm{Fe} 2-\mathrm{N} 3$ & $143.90(15)$ \\
\hline O7-Fe1-N1 & $92.12(15)$ & $\mathrm{O} 11-\mathrm{Fe} 2-\mathrm{N} 4$ & $146.16(14)$ \\
\hline O9-Fe1-N1 & $93.83(15)$ & O11-Fe1-N6 & $147.31(14)$ \\
\hline $\mathrm{O} 4-\mathrm{Fe} 2-\mathrm{O} 11$ & $96.64(13)$ & N2-Fe2-O2 & 149.60(13) \\
\hline $\mathrm{O} 10-\mathrm{Fe} 2-\mathrm{N} 2$ & $97.91(16)$ & N1-Fe1-O5 & $150.11(14)$ \\
\hline O7-Fe1-O11 & $98.39(14)$ & $\mathrm{O} 4-\mathrm{Fe} 2-\mathrm{O} 10$ & $163.23(14)$ \\
\hline $\mathrm{O} 11-\mathrm{Fe} 2-\mathrm{O} 10$ & $99.70(14)$ & O7-Fe1-O9 & $170.21(14)$ \\
\hline $\mathrm{C} 22-\mathrm{N} 6-\mathrm{Fe} 1$ & $103.7(3)$ & & \\
\hline
\end{tabular}




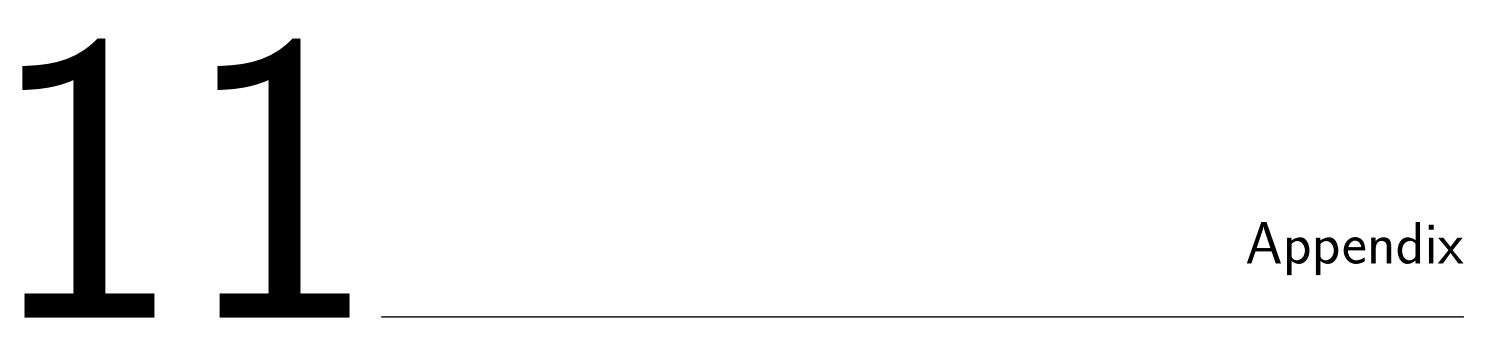

\subsection{Theory on Mössbauer Spectroscopy and SQUID Magnetometry}

Since this work exclusively focuses on iron based model chemistry, two particularly interesting methods, Mössbauer spectroscopy and SQUID magnetometry, will be discussed in this section. Aside from routine analytical methods these two techniques are well suited for the characterization of iron coordination compounds, but are not as widely established in common laboratories yet.

\subsubsection{Mössbauer Spectroscopy ${ }^{1}$}

The Mössbauer effect, first discovered by Rudolf L. Mößbauer in 1958, describes the recoilless nuclear resonance fluorescence of $\gamma$-radiation. 2201221. In the following years this observation was used to develop methods for the resonance spectroscopy of $\gamma$ rays at various nuclei in solids. 222 The principle of the Mössbauer effect that a part of the nuclear recoil energy is imparted onto an entire crystal lattice instead of a single atom, allowed for its employment in the spectroscopy of solids. Mössbauer spectroscopy allows for a non destructive chemical analysis of solids at low temperatures and is very sensitive to even small energy changes of $10^{-8} \mathrm{eV}$ (ca. $10^{-4} \mathrm{~cm}^{-1}$ ) for elements susceptible to the nuclear resonance phenomenon. In particular interesting is Mössbauer spectroscopy for ${ }^{57} \mathrm{Fe} \mathrm{nu}$ clei. For nuclear $\gamma$-resonance absorption to occur, the gamma radiation must be emitted by source nuclei of the same isotope as for the absorber. For ${ }^{57} \mathrm{Fe}$ nuclei generally the decay cascade of ${ }^{57} \mathrm{Co}$ is used to generate an excited ${ }^{57} \mathrm{Fe}^{*}$ isotope (Scheme 11.1 left).

In a recoilless emission and absorption of $\gamma$ rays, the emitted radiation from the ${ }^{57} \mathrm{Fe}^{*}$ excited state is absorbed by a second ${ }^{57} \mathrm{Fe}$ atom in a ground state (Scheme 11.1 right). Since the emitting source is usually embedded in a solid matrix (e.g. Rhodium) and the sample (absorber) in a crystal lattice a recoilless fraction should be sufficiently high. Additional cooling of the sample moreover enhances the resonance effect of the two nuclei. In a Mössbauer experiment the energy of the monochromatic $\gamma$-photons can be modulated using the Doppler effect. If either the radioactive source or the absorber is periodically moved with

\footnotetext{
${ }^{1}$ Unless otherwise noted most theories have been adapted from the very comprehensive textbook Mössbauer Spectroscopy and Transition Metal Chemistry by Philipp Gütlich, Eckhard Bill and Alfred X. Trautwein. 219
} 

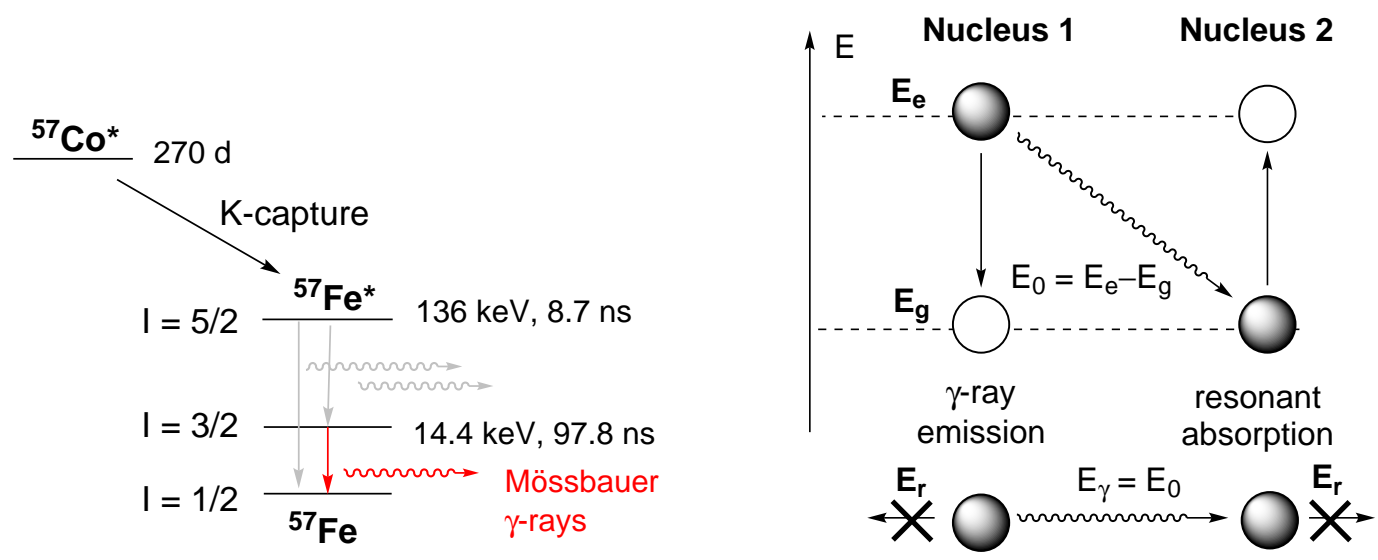

Scheme 11.1: Left: Decay cascade of ${ }^{57} \mathrm{Co}$ to generate an excited ${ }^{57} \mathrm{Fe}^{*}$ state. Right: Nuclear $\gamma$ resonance of two nuclei.

a certain velocity $+v$ forward or $-v$ backward the energy of the gamma photons changes according to $E_{\gamma}=E_{0}(1+v / \mathrm{c})$. Measuring the transmission of gamma rays as a function of the velocity a Mössbauer spectrum can be obtained. A maximum resonance occurs for an ideal overlap of absorption and emission lines. The greater the overlap of these lines, the lower is the transmission and hence detection of $\gamma$ rays. However, for the evaluation of a Mössbauer experiment mainly three interactions have to be considered. The Mössbauer nucleus is exposed to electric and magnetic fields of the electron cloud and hence the electric charge distribution and the magnetic dipole moment perturb the iron atoms' nuclear energy states. These so-called hyperfine interactions provide valuable information on physical and chemical properties of the sample (absorber) such as oxidation states, spin state, local symmetry and bonding properties such as covalency and electronegativity.

The isomer shift $\delta$, occurs due to the electric monopole interaction (Coulomb interaction) of a positively charged sphere (nucleus) with a cloud of electrons. This interaction depends directly on the s-electron densities (s-shells of the Mössbauer nucleus) at the nucleus, but can be influenced indirectly through shielding effects of p-, d- and f- electrons, which are usually not capable of influencing the nuclear field. 204 The electric monopole interaction effects the energy levels of the ground state and the excited state of a ${ }^{57} \mathrm{Fe}^{*}$ Mössbauer nucleus. These energy levels can be shifted by different amounts $(\delta \mathrm{E})_{\mathrm{g}}$ and $(\delta \mathrm{E})_{\mathrm{e}}$ compared to an unperturbed ${ }^{57} \mathrm{Fe}^{*}$ nucleus. Thus the maximum $\gamma$ resonance of the emitting source and absorber does not occur at the Doppler-velocity zero but is shifted, which is described by the parameter $\delta$ (Scheme 11.2).

The quadrupole splitting $\left|\Delta \boldsymbol{E}_{\mathrm{Q}}\right|$ in Mössbauer spectra results from an electric quadrupole interaction. This interaction originates from an interaction of a Mössbauer nucleus which possesses a quadrupole moment (for nuclei with $I>1 / 2$ ) and an electric field gradient (EFG). If the EFG at the nucleus differs from zero due to a non spherical valence electron distribution and/or deviations from cubic lattice site symmetry it can interact with the spin of the nucleus and cause a splitting into degenerate $\pm m_{\mathrm{i}}$ substates. For the first transition state of ${ }^{57} \mathrm{Fe}$ with $I=3 / 2$ a splitting into two substates 


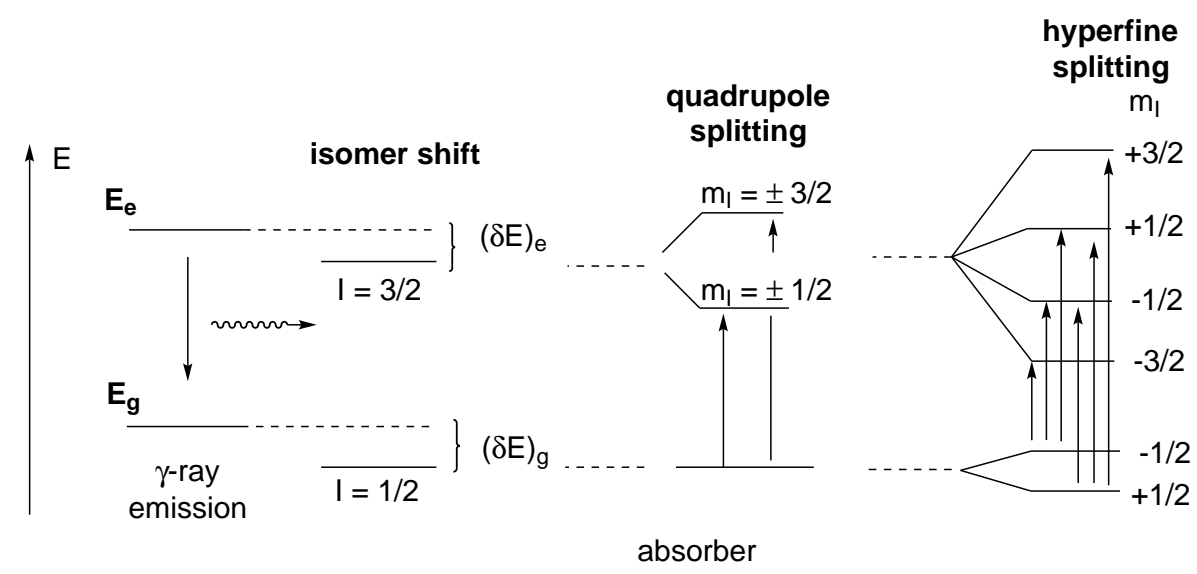

Scheme 11.2: Overview over the three main hyperfine interactions for evaluating physical and chemical properties of ${ }^{57} \mathrm{Fe}$ Mössbauer nuclei.

(Kramers doublets) with $I= \pm 3 / 2$ and $I= \pm 1 / 2$ would be visible (Scheme 11.2). Hence the quadrupole splitting parameter $\left|\Delta E_{\mathrm{Q}}\right|$, which is reflected in the line separation of a Mössbauer spectrum provides information about the bonding situation and symmetry of an iron atom.

A third hyperfine interaction is the magnetic hyperfine splitting interaction. Nuclei with a spin $\mathrm{I}>0$ generally can interact with an magnetic field through their magnetic dipole moment $\nu$. In this case the all states of an iron atom will be split in $2 \mathrm{n} I+1$ equally spaced non degenerate substates $m_{\mathrm{i}}$. This phenomenon is also known as the nuclear Zeeman effect. The magnetic dipole transitions can be predicted according to the following selection rules: $\Delta I=1, \Delta m_{\mathrm{i}}=0, \pm 1$. Hence for a ${ }^{57} \mathrm{Fe}$ atom with an $I=1 / 2$ ground state and an $I=3 / 2$ excited state six transition lines can be observed (Scheme 11.2). However, a magnetic hyperfine splitting can be observed if the nucleus creates a magnetic field of its own or if an external magnetic field is applied. By the hyperfine splitting the direction and size of the magnetic field influencing the Mössbauer nucleus can be determined.

\section{Implications of Mössbauer Spectroscopy in this Work}

The diiron coordination complexes synthesized in this work were investigated exclusively by zerofield Mössbauer spectroscopy. Relevant for the evaluation of the physical and chemical properties of these complexes are hence the isomer shift $\delta$ and the quadrupole splitting parameter $\left|\Delta E_{\mathrm{Q}}\right|$. Fe(II) and $\mathrm{Fe}(\mathrm{III})$ compounds with different spin states and their isomer shifts are well documented in literature $\underline{204219}$ and can together with the quadrupole splitting parameter be well distinguished. Not as trivial is the evaluation of Mössbauer parameters in iron and/or diiron nitrosyl compounds. According to RoDRIGUEz isomer shifts of $\{\mathrm{Fe}(\mathrm{NO})\}^{7}$ sites can not be easily classified as common iron oxidation states. 223 , For $S=3 / 2$ spin states, isomer shifts are reduced with respect to the high spin ferrous and enhanced with respect to the high spin ferric configurations. ${ }^{81 \mid 223}$ For $S=1 / 2\{\mathrm{Fe}(\mathrm{NO})\}^{7}$ sites isomer shifts can according to WIEGHARDT differ greatly. [176]177] As there are no diiron dinitrosyl model systems with $S=1 / 2$ spin state known to date, only mononuclear 
analogues can be used for comparison. In some of these model systems with $N$-donating ligands the isomer shift varies between $\delta=0.2-0.4 \mathrm{mms}^{-1}$. et al. Mössbauer spectroscopy can only serve as indirect reporter for bonding properties and spin state of iron nitrosyl and dinitrosyl complexes as it probes only the electron density of the iron nucleus. ${ }^{225]}$ Table 11.1 lists all Mössbauer parameters for the investigated complexes in this work along with the iron oxidation state.

Table 11.1: Mössbauer parameters for diiron complexes in this work.

\begin{tabular}{|c|c|c|c|c|c|}
\hline compund & $\delta\left[\mathrm{mms}^{-1}\right]$ & $\left|\Delta E_{\mathrm{Q}}\right|\left[\mathrm{mms}^{-1}\right]$ & $\Gamma_{\mathrm{FWHM}}$ & rel intensity [\%] & Ox. state \\
\hline \multirow[t]{2}{*}{$1 \mathrm{a}$} & 0.45 & 0.16 & 0.33 & 42 & $\mathrm{Fe}(\mathrm{II}) \mathrm{ls}$ \\
\hline & 0.98 & 2.79 & 0.33 & 58 & $\mathrm{Fe}(\mathrm{II}) \mathrm{hs}$ \\
\hline \multirow[t]{2}{*}{$\mathbf{1 a}$ in $\mathrm{MeCN}$} & 0.40 & 0.10 & 0.39 & 46 & $\mathrm{Fe}(\mathrm{II}) \mathrm{ls}$ \\
\hline & 1.01 & 2.88 & 0.36 & 53 & $\mathrm{Fe}(\mathrm{II}) \mathrm{hs}$ \\
\hline \multirow[t]{2}{*}{$\mathbf{1 a}$ in $\mathrm{EtCN}$} & 0.43 & 0.20 & 0.34 & 45 & $\mathrm{Fe}(\mathrm{II})$ ls \\
\hline & 1.00 & 2.92 & 0.34 & 55 & $\mathrm{Fe}(\mathrm{II}) \mathrm{hs}$ \\
\hline \multirow[t]{2}{*}{$1 b$} & 1.19 & 2.52 & 0.39 & 49 & $\mathrm{Fe}(\mathrm{II}) \mathrm{hs}$ \\
\hline & 1.20 & 3.14 & 0.39 & 51 & $\mathrm{Fe}(\mathrm{II}) \mathrm{hs}$ \\
\hline \multirow[t]{2}{*}{$1 b^{\text {Int }}$} & 0.49 & 0.72 & 0.42 & 44 & Fe(III) hs \\
\hline & 1.10 & 3.01 & 0.41 & 56 & $\mathrm{Fe}(\mathrm{II}) \mathrm{hs}$ \\
\hline \multirow[t]{2}{*}{$1 b^{O x}$} & 0.47 & 0.89 & 0.45 & 73 & $\mathrm{Fe}(\mathrm{III}) \mathrm{hs}$ \\
\hline & 0.54 & 1.84 & 0.45 & 27 & $\mathrm{Fe}(\mathrm{III}) \mathrm{hs}$ \\
\hline $2 a$ & 0.43 & 0.18 & 0.33 & 100 & $\mathrm{Fe}(\mathrm{II}$ ls \\
\hline \multirow[t]{2}{*}{$2 b$} & 0.43 & 0.18 & 0.36 & 79 & $\mathrm{Fe}(\mathrm{II}$ ls \\
\hline & 1.10 & 3.08 & 0.55 & 21 & $\mathrm{Fe}(\mathrm{II}$ hs \\
\hline \multirow[t]{2}{*}{3} & 0.45 & 1.11 & 0.56 & 52 & $\mathrm{Fe}(\mathrm{III}) \mathrm{hs}$ \\
\hline & 0.49 & 0.67 & 0.38 & 48 & $\mathrm{Fe}(\mathrm{III}) \mathrm{hs}$ \\
\hline \multirow[t]{3}{*}{4} & 0.62 & 1.65 & 0.29 & 47 & $\{\mathrm{Fe}(\mathrm{NO})\}^{7}$ \\
\hline & 0.61 & 1.85 & 0.29 & 47 & $\{\mathrm{Fe}(\mathrm{NO})\}^{7}$ \\
\hline & 1.17 & 3.31 & 0.39 & 6 & impurity \\
\hline \multirow[t]{2}{*}{$4^{\text {Red }}$} & 1.04 & 2.59 & 0.28 & 50 & $\mathrm{Fe}(\mathrm{II}) \mathrm{hs}$ \\
\hline & 1.11 & 2.86 & 0.28 & 50 & $\mathrm{Fe}(\mathrm{II}) \mathrm{hs}$ \\
\hline \multirow[t]{2}{*}{5} & 0.27 & 0.95 & 0.38 & 50 & $\{\mathrm{Fe}(\mathrm{NO})\}^{7}$ \\
\hline & 0.63 & 1.95 & 0.38 & 50 & $\{\mathrm{Fe}(\mathrm{NO})\}^{7}$ \\
\hline \multirow[t]{2}{*}{$5^{\text {Red }}$} & 1.08 & 2.80 & 0.31 & 85 & $\mathrm{Fe}(\mathrm{II}) \mathrm{hs}$ \\
\hline & 0.42 & 0.56 & 0.35 & 15 & impurity \\
\hline 7 & 0.57 & 0.57 & 0.55 & 100 & $\mathrm{Fe}(\mathrm{III}) \mathrm{hs}$ \\
\hline
\end{tabular}




\subsubsection{SQUID Magnetometry}

The magnetic susceptibility measurements in this work (temperature dependence as well as variable temperature variable field (VTVH) measurements) were recorded via SQUID (Superconducting Quantum Interference Device) magnetometry. The magnetic susceptibility describes a dependency of the magnetization $M$ of a sample from an external magnetic field $H$. SQUID magnetometer are sensitive enough to measure extremely subtle electromagnetic fields $\left(5 \times 10^{-15} \mathrm{~T}\right)$ and are hence well suited to examine the magnetization of very low amounts of a magnetic material. $\stackrel{226}{2}$. A central component of a SQUID magnetometer is a superconducting loop with a so-called weak link. The closed superconducting loop is often made of a niobium alloy. Superconducting materials such as these alloys exhibit a complete lack of electrical resistance below a certain transition temperature. The superconducting loop however is interrupted at one position/junction and the superconducting material is replaced by an insulator. 227. The size of this "gap" usually lies in the range of a few nanometers. This junction (superconductor-insulator-superconductor) is more commonly known as Josephson junction. The barrier (Josephson junction) for the electric current in the superconducting material can be overcome by the tunneling (Cooper electron pairs) of electrons. A small tunneling current can be detected. If a small external magnetic field is applied to a superconducting loop, a screening current starts circulating through the loop, compensating the external flux, also known as the Meissner effect. 227. This is flux is quantized and can only be described by integer multiples of the magnetic flux quantum. The tunneling current within the Josephson junction is very sensitive to the applied external field. Small variations of the magnetic field can lead to a temporarily collapse of the superconducting current, which can be detected. The measurement of a sample in a SQUID magnetometer is realized only indirectly. The sample is moved through a system of superconducting detection coils, which are connected to the superconduction ring (SQUID) with superconducting wires. The detection coils couple inductively with the SQUID sensor. Hence the magnetic moment of the sample induces an electric current in the detection coils at a given temperature which is passed on to the SQUID and detected. 227. As the sample moves through the coils the magnetic dipole moment of the sample induces an electric current in the detection coils. The SQUID functions as a highly linear current-to-voltage converter, so that variations in the current of the detection coil circuit produce corresponding variations in the SQUID output voltage as a function of the direction of the sample movement and its position with respect to the coil. 228, A basic setup of a SQUID magnetometer and the respective output signal are depicted in Figure 11.1

The output signal can be transformed to obtain a value for the magnetization $M$ of the sample at an applied magnetic field $H$ at a given temperature. 230]

$$
M=\chi_{\mathrm{V}} \cdot H
$$

The volume magnetic susceptibility of the sample resembles the proportionality factor between $M$ and $H$ and describes the response of the sample to the applied magnetic field. The volume magnetic susceptibility is a dimensionless figure and can be converted to a molar magnetic susceptibility according to 231]: 


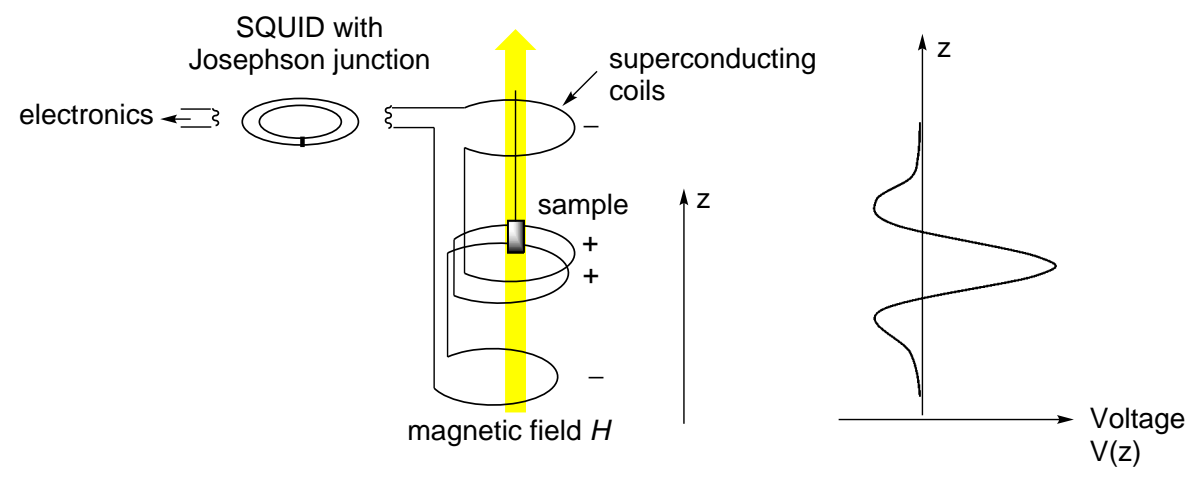

Figure 11.1: Left: Setup of a SQUID magnetometer with one Josephson junction. The sample is moved along the z-axis, parallel to the applied magnetic field. Right: Output signal detected by the SQUID. Respective potential in dependency of the position of the sample within the detection coils. 229.

$$
\chi_{\mathrm{m}}=\chi_{\mathrm{V}} \cdot \frac{V}{\mathrm{n}}\left[\mathrm{cm}^{3} / \mathrm{mol}\right]
$$

The temperature dependence of the molar magnetic susceptibility in 3d metals can usually be described by the Curie law in its spin only form assuming that the orbital angular momentum for these metals is close to zero. 230,232

$$
\chi_{\mathrm{m}}=\frac{\mathrm{N}_{\mathrm{A}}}{3 \mathrm{k}_{\mathrm{B}} T} \mu_{\mathrm{B}}^{2} \mathrm{~g}_{\mathrm{e}}^{2} \sum S_{\mathrm{i}}\left(S_{\mathrm{i}}+1\right)=\frac{\mathrm{C}}{T}
$$

$\mathrm{N}_{\mathrm{A}} \quad$ Avogadros constant

$\mathrm{k}_{\mathrm{B}} \quad$ Boltzmann constant

$\mu_{\mathrm{B}} \quad$ Bohr magneton

ge Landé factor

$S \quad$ spin

The Curie constant $\mathrm{C}$ is can be derived from quantum mechanical considerations via the van Vleck equation 230, which includes the Eigenvalues and Eigenfunctions from perturbation theory and will not be discussed in detail in this respect.

Generally, for the evaluation of a SQUID experiment it should be noted that the measured susceptibility contains three main contributions which are

$$
\chi=\chi_{\text {para }}+\chi_{\text {dia }}+\chi_{\mathrm{TIP}}
$$

In this respect $\chi_{\text {dia }}$ describes diamagnetic temperature independent contributions which result from the experimental setup whereas $\chi_{\text {TIP }}$ is a pure orbital momentum effect. Hence, measurements are usually corrected for these two contributions.

The molar magnetic susceptibility is moreover closely related to the effective magnetic moment $\mu_{\text {eff }}$. Its relation with the magnetic susceptibility for spin only systems can be described by the following equation 23012321233 :

$$
\frac{\mu_{\mathrm{eff}}}{\mu_{\mathrm{B}}}=\sqrt{\frac{3 \mathrm{k}_{\mathrm{B}}}{\mathrm{N}_{\mathrm{A}} \mu_{\mathrm{B}}^{2}}} \sqrt{\chi_{\mathrm{m}} T}=\mathrm{g}_{\mathrm{e}} \sqrt{S(S+1)}
$$


To describe magnetism in polynuclear molecular compounds, which do not behave according to the idealized model of a paramagnet the magnetic coupling of paramagnetic centers has to be considered. In this respect the intramolecular exchange coupling via superexchange plays a most important role.

The intramolecular exchange coupling of paramagnetic centers for isotropic exchange can be described phenomenologically by the Heisenberg-Dirac-van-Vleck-Hamiltonian. For a system of two magnetic centers with a spin $S$ the Hamiltonian could be described as 230231 :

$$
\hat{H}=-2 J \hat{S}_{1} \hat{S}_{2}
$$

In this context $J$ describes the coupling constant of the two magnetic iron centers. The magnitude of $J$ is important for the evaluation whether the two iron sites are ferromagnetically or antiferromagnetically coupled. For ferromagnetically coupled centers the magnetic spins of both iron sites point in the same direction $(J>0)$, whereas for antiferromagnetically coupled centers the magnetic spins point in opposite directions $(\mathrm{J}<0)$. For the evaluation of the measured data further effects were included in the Hamiltonian such as zerofield and Zeeman splitting so that the full Hamiltonian can be described as follows:

$$
\begin{gathered}
\hat{H}=\hat{H}^{\mathrm{exc}}+\hat{H}^{\mathrm{ZFS}}+\hat{H}^{\mathrm{Zeem}} \\
\hat{H}^{\mathrm{exc}}=-2 \sum_{i>j} J_{i j} \hat{S}_{i} \hat{S}_{j} \\
\hat{H}^{\mathrm{ZFS}}=\sum_{i} D_{i}\left(\hat{S}_{z, i}^{2}-\frac{1}{3} S_{i}\left(S_{i}+1\right) \frac{E_{i}}{D_{i}}\left(\hat{S}_{x, i}^{2}-\hat{S}_{y, i}^{2}\right)\right) \\
\hat{H}^{\mathrm{Zeem}}=\sum_{i} \mu_{\mathrm{B}}\left(\hat{S}_{x, i} g_{x, i} B_{x}+\hat{S}_{y, i} g_{y, i} B_{y}+\hat{S}_{z, i} g_{z, i} B_{z}\right)
\end{gathered}
$$

Mechanistically, the intramolecular exchange coupling can occur direct via the spin orbitals of two metal sites in very close proximity or indirect via orbitals of a bridging ligand, which is often referred to as superexchange. 234 Different concepts were developed to qualitatively explain the superexchange mediated by bridging ligand orbitals. 230|232] An important parameter that describes the exchange coupling in this respect is the overlap integral of two magnetic orbitals. If the overlap integral is zero, the magnetic orbitals of the two magnetic centers as well as the orbitals of the bridging ligand are orthogonal to each other and a ferromagnetic interaction can be expected. For overlap integrals between zero and one, a partial or full overlap of the magnetic orbitals can be expected. Hence these magnetic centers interact antiferromagnetically. 235

For the investigated pyrazolate bridged dinuclear iron complexes in this work typically weak antiferromagnetic coupling is experimentally observed. Individual overlap integrals were not calculated for the investigated complexes. 


\subsection{Supplementary Material for the Characterization of the Diferrous Complexes}

\subsubsection{NMR and UV/Vis Studies of 2a}

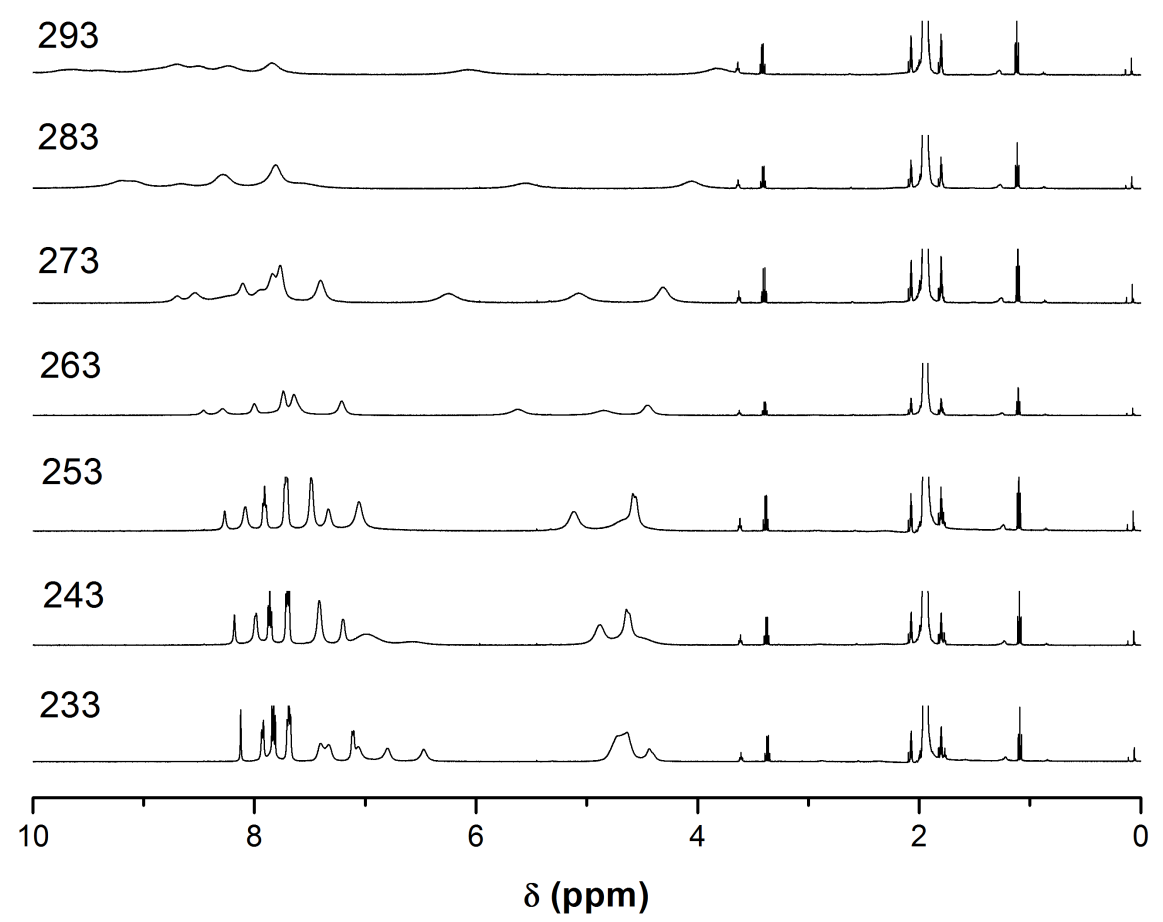

Figure 11.2: Variable temperature NMR spectra of $\mathbf{2 a}$ in $\mathrm{MeCN}-\mathrm{d}_{3}$. With decreasing temperature signals sharpen indicating a higher low spin contribution.

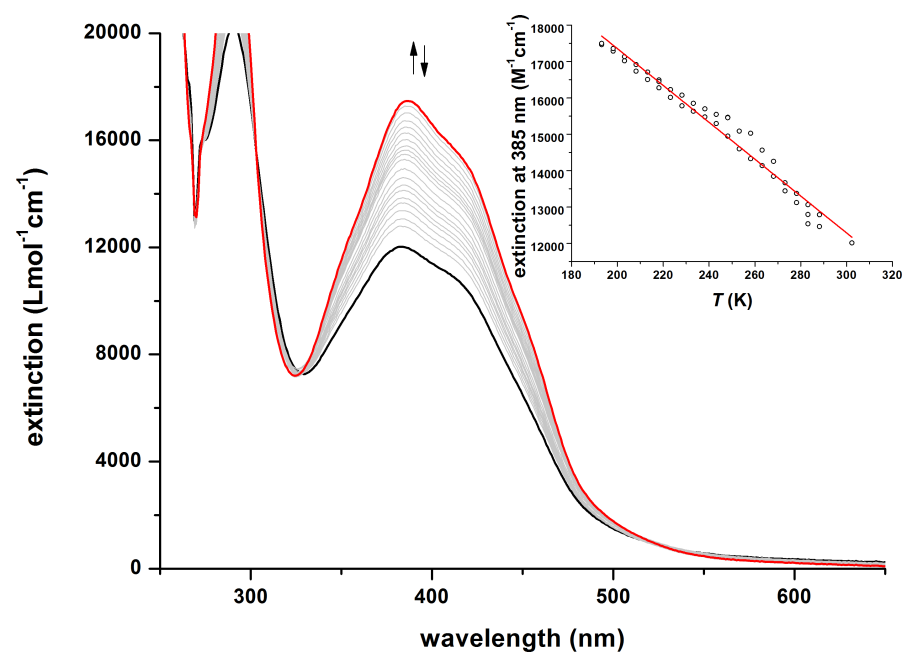

Figure 11.3: Temperature dependent UV/Vis spectrum of 2a. Increase of the extinction coefficient at $386 \mathrm{~nm}$ upon cooling and decrease upon warming. Inset: Temperature trace of the extinction. 


\subsubsection{ESI Mass Spectra}

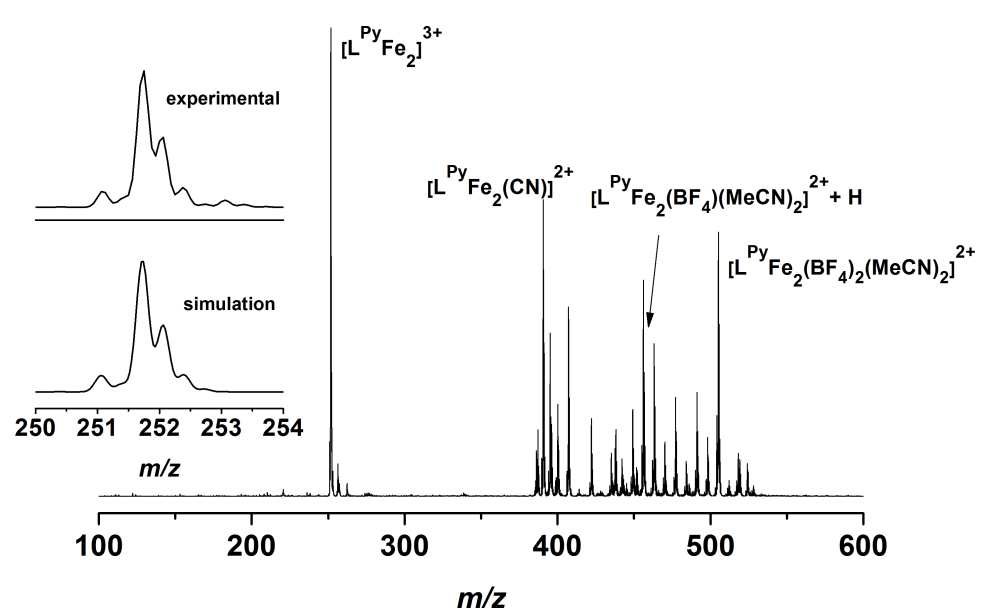

Figure 11.4: $\mathrm{ESI}^{+}$mass spectrum of $\mathbf{2 b}$ in $\mathrm{MeCN}$. Not all signals can be assigned with certainty.

\subsubsection{Mössbauer spectra}
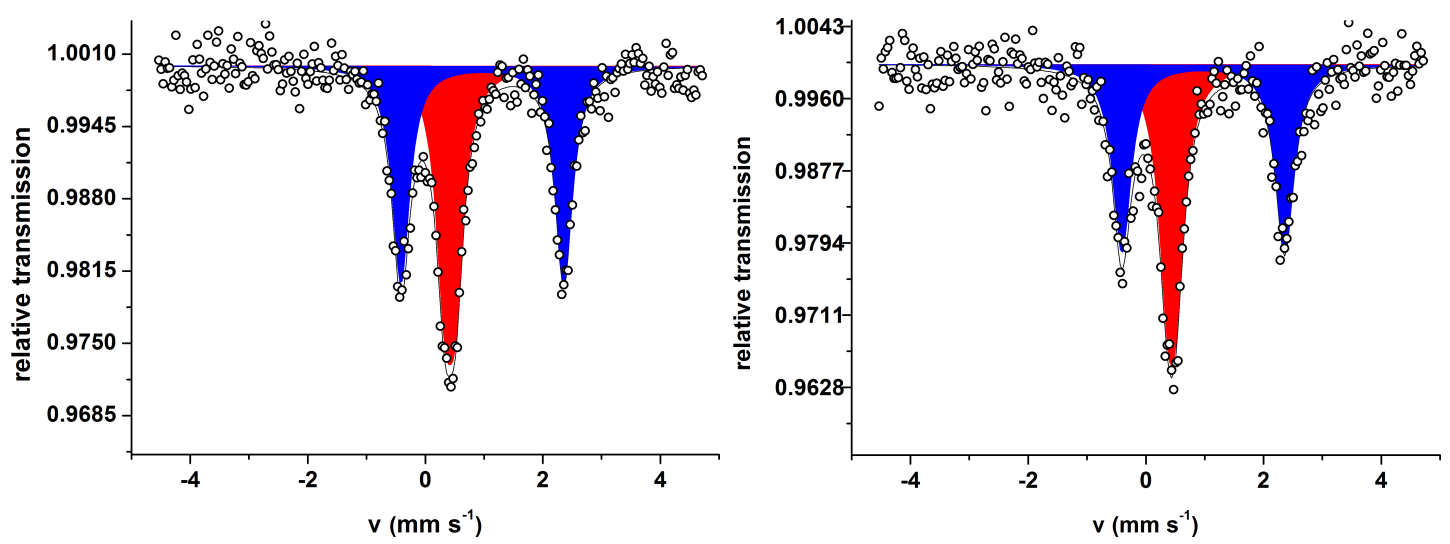

Figure 11.5: Mössbauer spectra of a crystalline sample of $\left[\mathrm{L}^{\mathrm{Im}} \mathrm{Fe}\{\mathrm{Fe}(\mathrm{MeCN})\}\right](\mathrm{OTf})_{3}$ 1a. Isomer shifts $\delta$ and quadrupole splittings $\left|\Delta E_{\mathrm{Q}}\right|$ in $\left[\mathrm{mm} \mathrm{s}^{-1}\right]$. Left: At $\mathbf{8 0 ~ K}$, red subspectrum $\delta=0.42$, $\left|\Delta E_{\mathrm{Q}}\right|=0.18$, blue subspectrum $\delta=0.97,\left|\Delta E_{\mathrm{Q}}\right|=2.77$. Right: At $\mathbf{6} \mathbf{K}$, red subspectrum $\delta=0.44$, $\left|\Delta E_{\mathrm{Q}}\right|=0.13$, blue subspectrum $\delta=0.98,\left|\Delta E_{\mathrm{Q}}\right|=2.77$. 


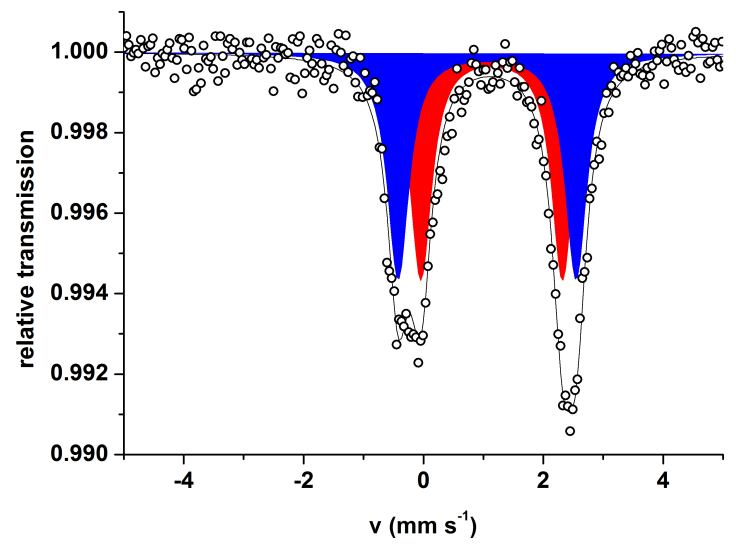

Figure 11.6: Mössbauer spectrum of a vacuum dried crystalline sample of $\left[\mathrm{L}^{\operatorname{Im}} \mathrm{Fe}\{\mathrm{Fe}(\mathrm{MeCN})\}\right](\mathrm{OTf})_{3} \mathbf{1 a}$ at $\mathbf{8 0} \mathbf{K}$. Isomer shift $\delta$ and quadrupole splitting $\left|\Delta E_{\mathrm{Q}}\right|$ in [mm s$\left.{ }^{-1}\right]$. Red subspectrum: $\delta=1.14,\left|\Delta E_{\mathrm{Q}}\right|=2.36$, blue subspectrum: $\delta=1.01,\left|\Delta E_{\mathrm{Q}}\right|=2.97$.
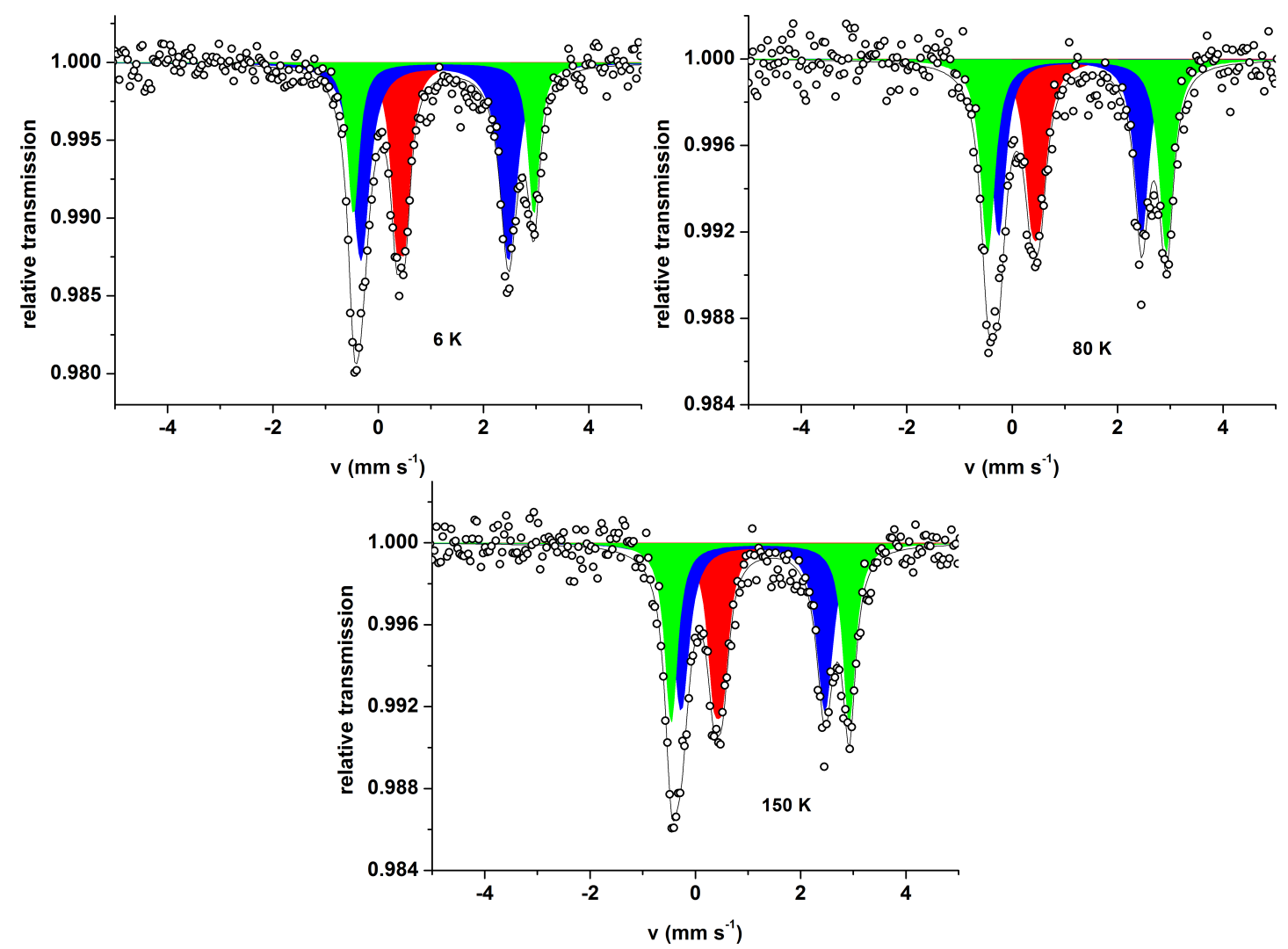

Figure 11.7: Frozen solution Mössbauer spectra of $\mathbf{1 b}$ in EtCN. Isomer shifts $\delta$ and quadrupole splittings $\left|\Delta E_{\mathrm{Q}}\right|$ in $\left[\mathrm{mm} \mathrm{s}^{-1}\right]$. Left: At $6 \mathbf{K}$, red subspectrum $\delta=0.43,\left|\Delta E_{\mathrm{Q}}\right|=0.17$, blue subspectrum $\delta=1.07,\left|\Delta E_{\mathrm{Q}}\right|=2.80$, green subspectrum $\delta=1.24,\left|\Delta E_{\mathrm{Q}}\right|=3.43$. Right: At $80 \mathbf{K}$, red subspectrum $\delta=0.45,\left|\Delta E_{\mathrm{Q}}\right|=0.17$, blue subspectrum $\delta=1.10,\left|\Delta E_{\mathrm{Q}}\right|=2.71$, green subspectrum $\delta=1.23,\left|\Delta E_{\mathrm{Q}}\right|=3.39$. Bottom: At $\mathbf{1 5 0 \mathbf { K }}$, red subspectrum $\delta=0.43,\left|\Delta E_{\mathrm{Q}}\right|=0.17$, blue subspectrum $\delta=1.09,\left|\Delta E_{\mathrm{Q}}\right|=2.73$, green subspectrum $\delta=1.24,\left|\Delta E_{\mathrm{Q}}\right|=3.38$. 


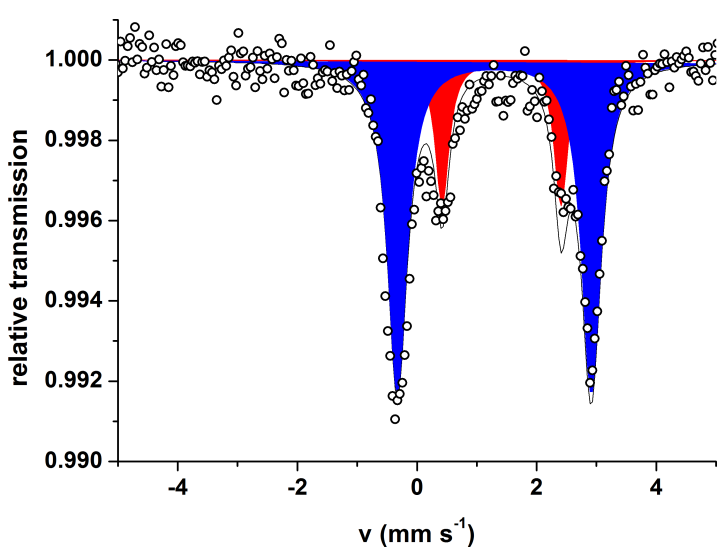

Figure 11.8: Frozen solution Mössbauer spectrum of $\mathbf{1 b}$ in MeCN at $80 \mathrm{~K}$. Isomer shifts $\delta$ and quadrupole splittings $\left|\Delta E_{\mathrm{Q}}\right|$ in $\left[\mathrm{mm} \mathrm{s}^{-1}\right]$. Red subspectrum $\delta=1.41,\left|\Delta E_{\mathrm{Q}}\right|=1.99$, blue subspectrum $\delta=1.29,\left|\Delta E_{\mathrm{Q}}\right|=3.24$. Integral ratio [\%]: 24:76. 


\subsection{Supplementary Material for the Reactivity Studies of the Diiron Core with Dioxygen}

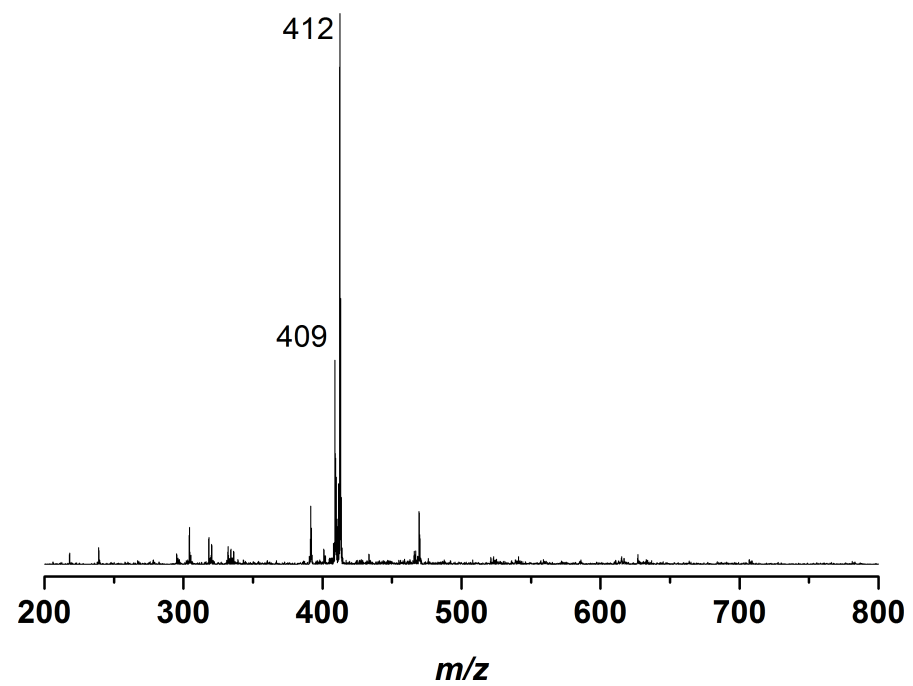

Figure 11.9: $\mathrm{ESI}^{+}$mass spectrum of $\mathbf{1 a}^{\mathrm{Ox}}$ in MeCN. Most signals could not be assigned.

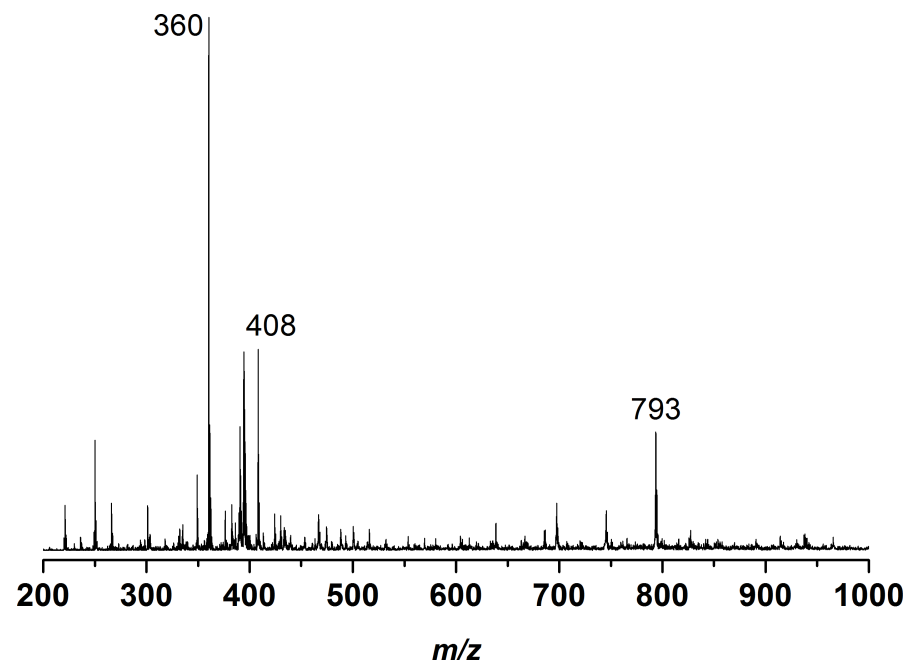

Figure 11.10: $\mathrm{ESI}^{+}$mass spectrum of $\mathbf{2 \mathbf { a } ^ { \mathbf { O } }}$ in MeCN. Most signals could not be assigned. 


\subsection{Supplementary Material for the Characterization of Diiron Dinitrosyl Complexes}

\subsubsection{Crystal structure of $4 b^{\text {Red }}$}

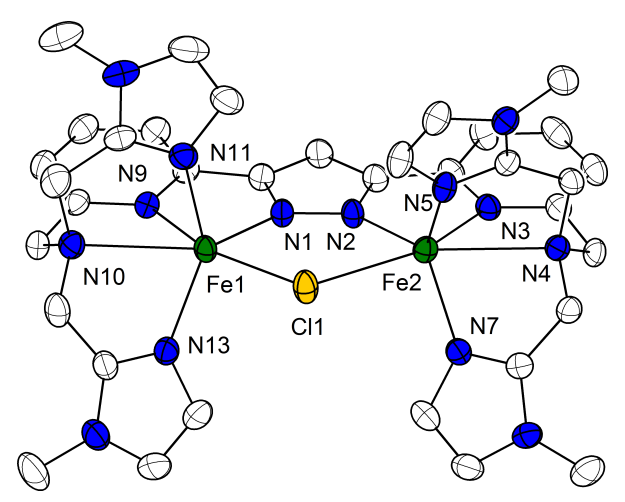

Figure 11.11: Crystal structure of the cation of $\left[\mathrm{L}^{\mathrm{Im}} \mathrm{Fe}_{2}(\mu-\mathrm{Cl})\right]\left(\mathrm{SO}_{3} \mathrm{CF}_{3}\right)_{2} ; 2 \mathrm{DCM} \mathbf{4} \mathbf{b}^{\text {Red }}$ with $50 \%$ probability ellipsoids. Hydrogen atoms, triflate counterions and solvent molecules were omitted for clarity. Selected bond lengths $[\AA]$ and angles $\left[{ }^{\circ}\right]$ : Fe1-N1 2.080(2), Fe1-N9 2.206(2), Fe1N9 2.206(2), Fe1-N10 2.610(2), Fe1-N11 2.104(2), Fe1-N13 2.087(2), Fe1-Cl1 2.4886(8), Fe2-N2 2.095(2), Fe2-N3 2.252(2), Fe2-N4 2.570(2), Fe2-N5 2.093(2), Fe2-N7 2.070(2), Fe2-Cl1 2.7202(8), Fe1-Cl1-Fe2 107.63(3), Fe1-N1-N2-Fe2 -1.460(4).

\subsubsection{IR Spectrum of $4^{\text {Red }}$}

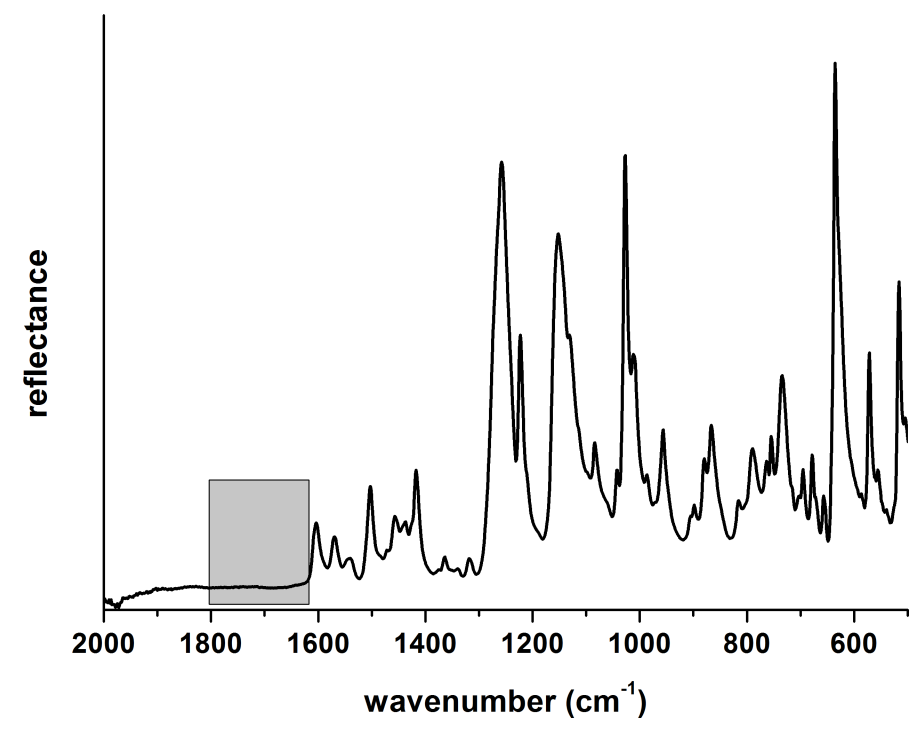

Figure 11.12: ATR spectrum of $4^{\text {Red }}$. The gray box indicates a region in which NO stretches of the precursor would appear. 


\subsubsection{ESI Mass Spectra}

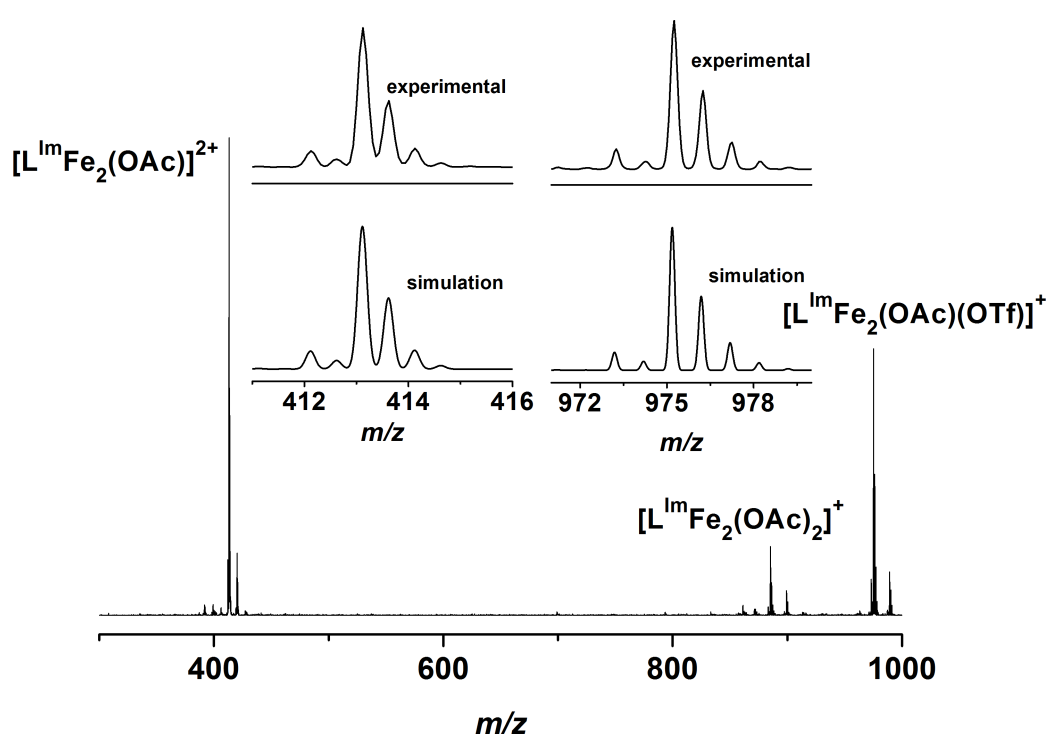

Figure 11.13: $\mathrm{ESI}^{+}$mass spectrum of 4 in $\mathrm{MeOH}$.

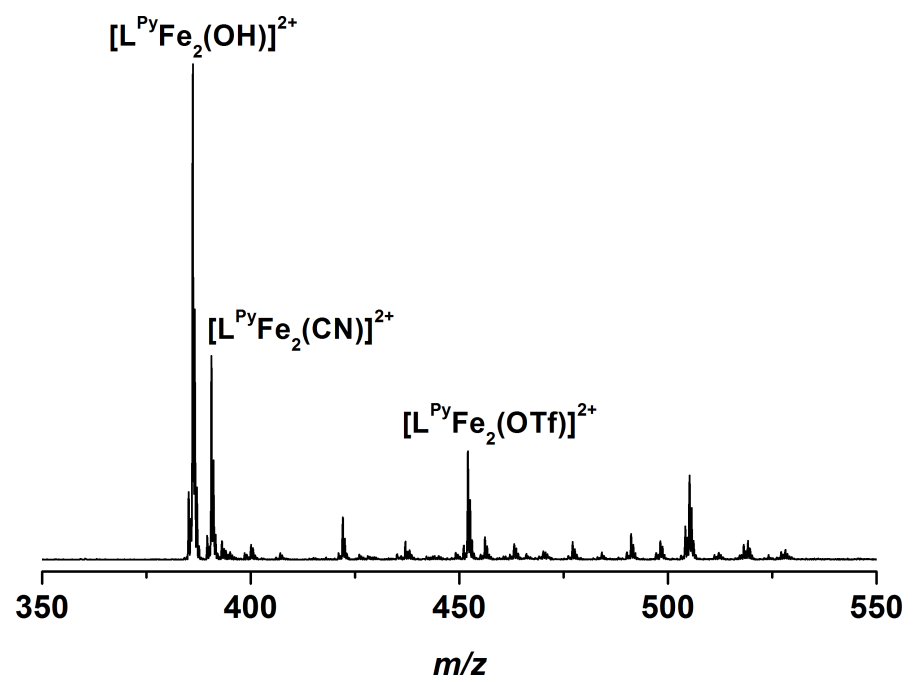

Figure 11.14: $\mathrm{ESI}^{+}$mass spectrum of 5 in $\mathrm{MeOH}$. 


\subsubsection{Labelling Studies with 4}

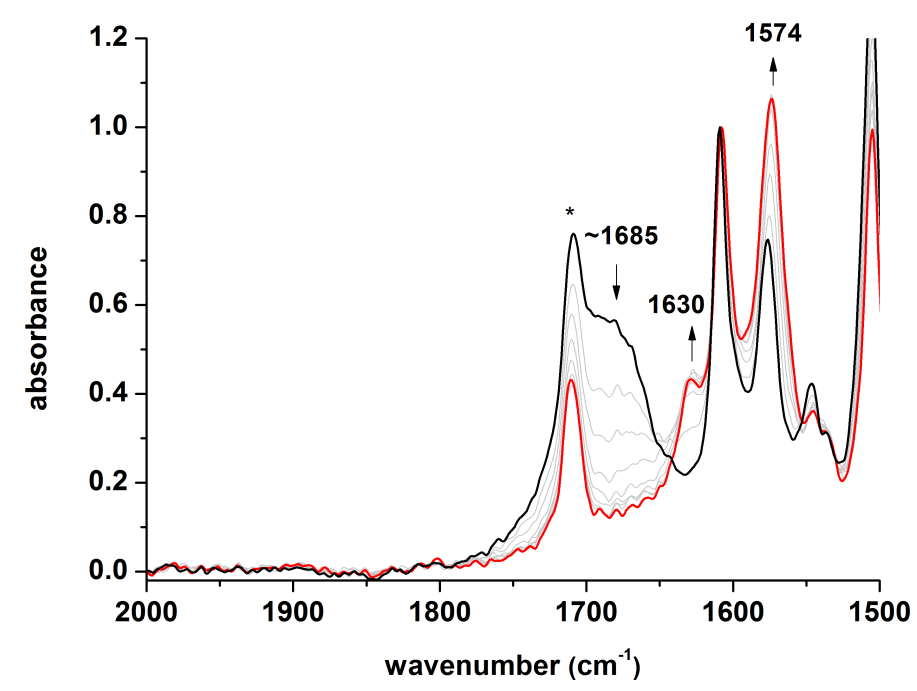

Figure 11.15: Electrochemical reduction at an applied potential of $-1200 \mathrm{mV}$ of ${ }^{15} \mathrm{~N}^{18} \mathrm{O}$ labeled 4 in DCM/MeOH 5:1 followed by IR spectroscopy. The NO-band at $1685 \mathrm{~cm}^{-1}$ decreases while new bands at $1630 \mathrm{~cm}^{-1}$ and $1574 \mathrm{~cm}^{-1}$ appear. The asterisk marks an impurity in the solvent.

\subsubsection{UV/Vis Spectra of 4}
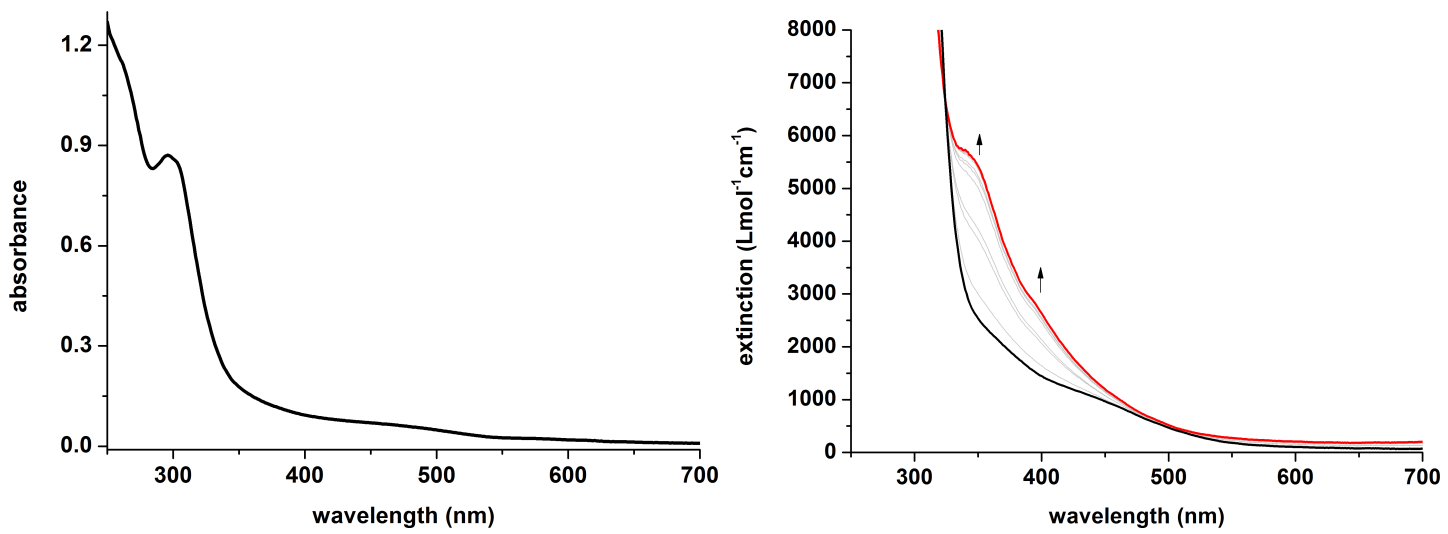

Figure 11.16: UV/Vis spectra of 4. Left: Spectrum of 4 in $\mathrm{DCM} / \mathrm{MeOH}$ at $20^{\circ} \mathrm{C}$. Right: Formation of $\mathbf{4}$ (red line) upon addition of $\mathrm{NO}$ to a solution of $\mathbf{1 a}$ (black line) in $\mathrm{MeOH}$ at $-80^{\circ} \mathrm{C}$. 


\subsubsection{UV/Vis Spectra of Cobaltocene}
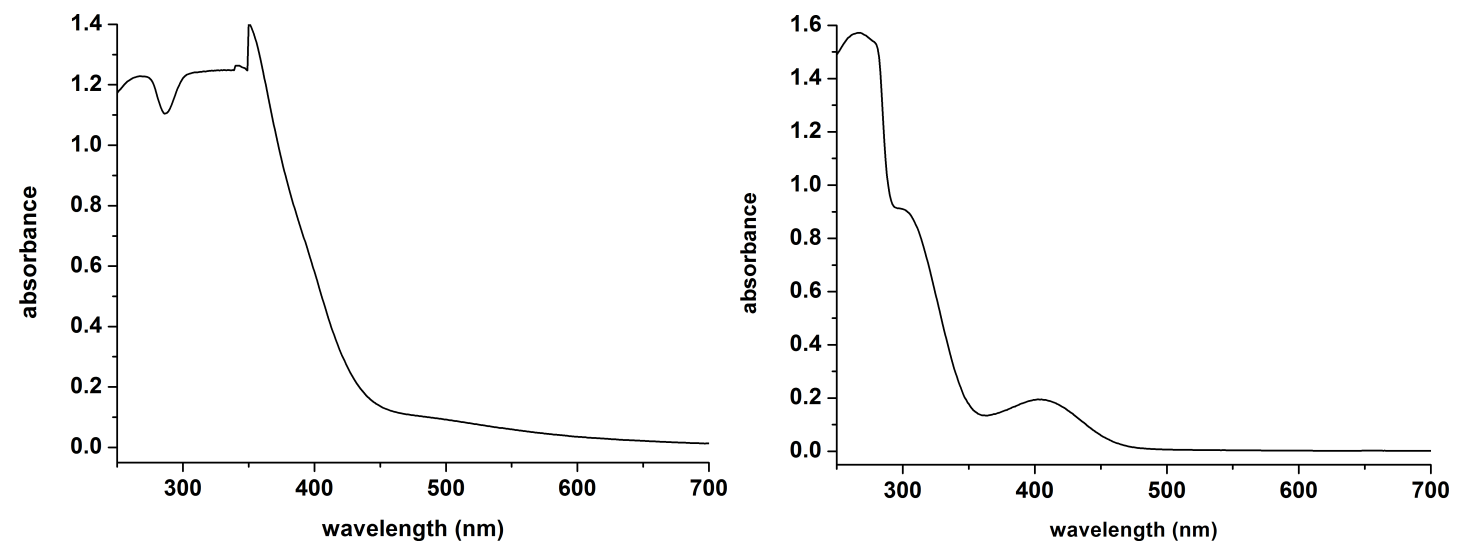

Figure 11.17: UV/Vis spectra of cobaltocene (left) and oxidized cobaltocene (right) in $\mathrm{DCM} / \mathrm{MeOH}$ 5:1.

\subsubsection{Setup for IR Headspace Analyses}

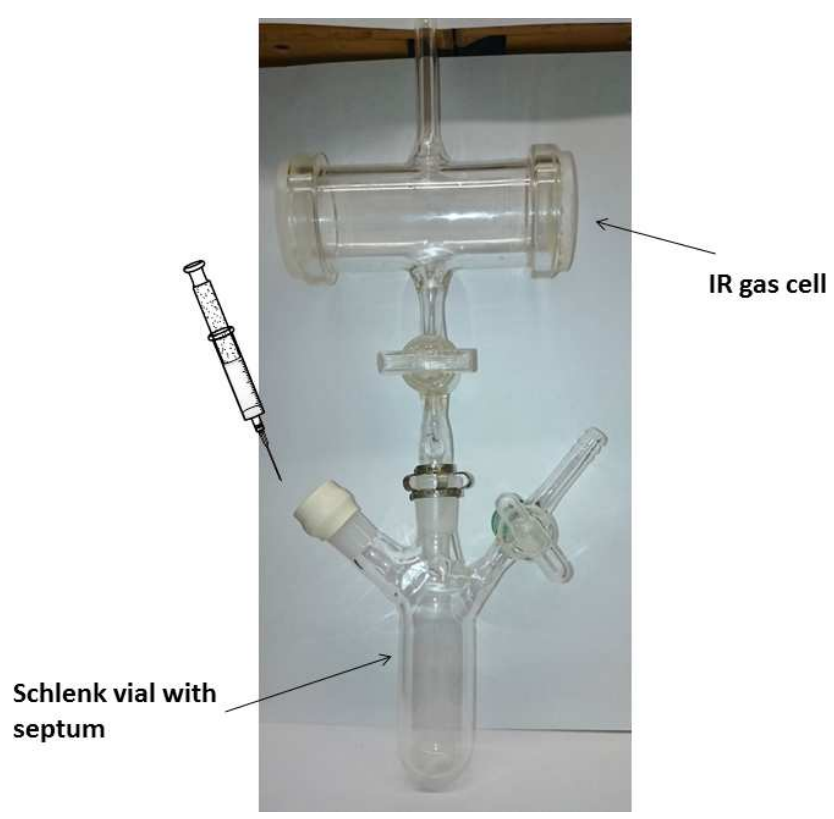

Figure 11.18: Setup for the $\mathrm{N}_{2} \mathrm{O}$ headspace analyses. 


\subsection{Supplementary Material for the Excursus on Water Oxidation Catalysis.}

\subsubsection{ESI Mass Spectra}

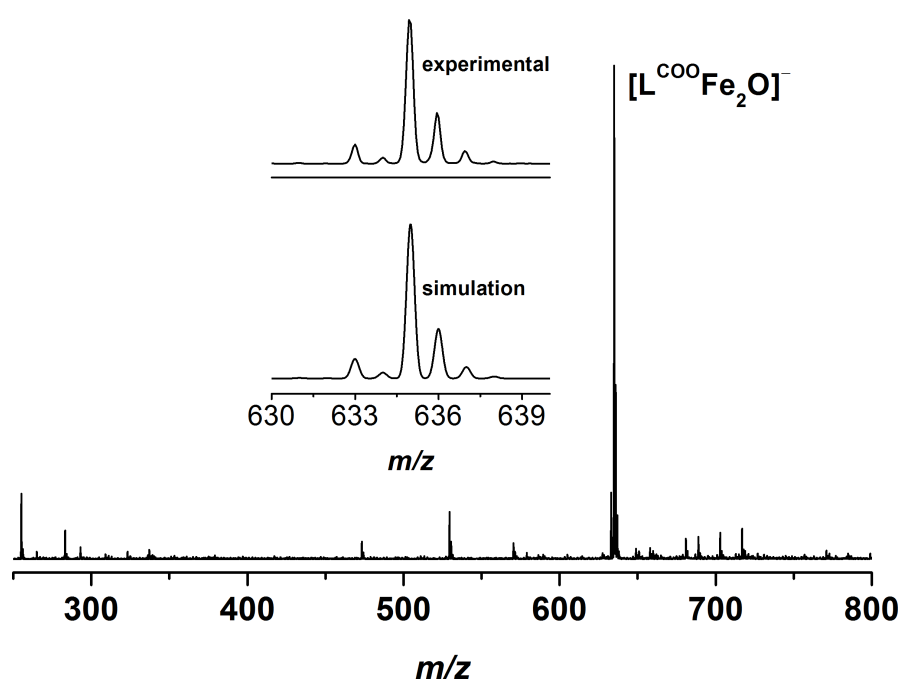

Figure 11.19: $\mathrm{ESI}^{-}$mass spectrum of 6 in $\mathrm{MeOH}$.

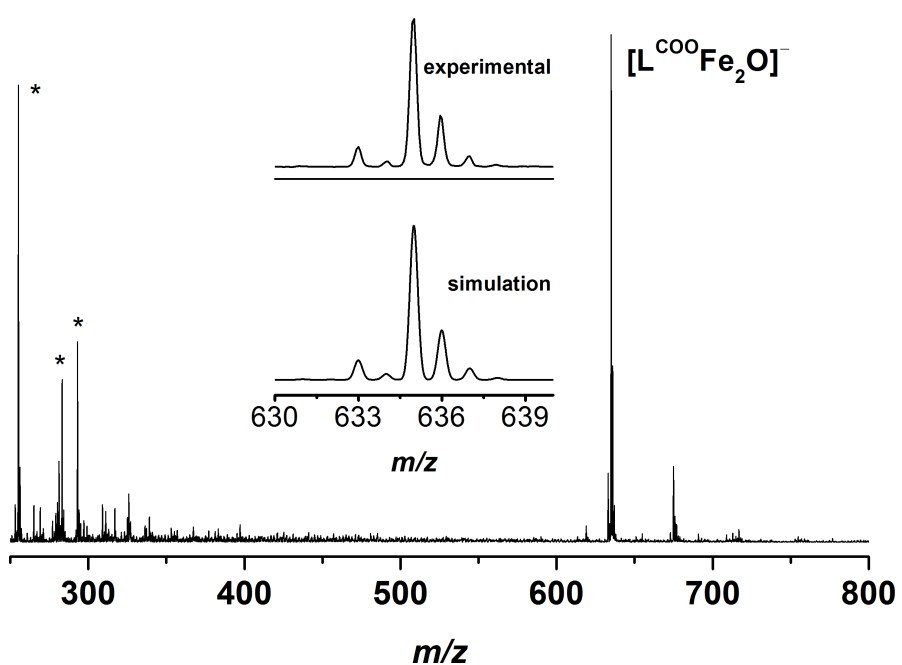

Figure 11.20: $\mathrm{ESI}^{-}$mass spectrum of 7 in $i \mathrm{PrOH} / \mathrm{H}_{2} \mathrm{O} 1 / 1$. The asterisk marks signals which stem from the solvent or residues inside the spectrometer. 


\subsection{Abbreviations}

\begin{tabular}{|c|c|}
\hline ATR & attenuated total reflectance \\
\hline AurF & $p$-aminobenzoate $N$ oxygenase \\
\hline BPEAN & 1,8-naphthyridine-based ligand \\
\hline $\mathrm{Bpz}_{3}$ & trispyrazoylborate \\
\hline CAN & ceric ammonium nitrate \\
\hline $\mathrm{CmII}$ & chloramphenicol $N$ oxygenase \\
\hline DCM & dichloromethane \\
\hline $\mathrm{DMF}$ & dimethylformamide \\
\hline DNIC & dinitrosyl iron complexes \\
\hline EFG & electric field gradient \\
\hline EI & electron ionization \\
\hline EPR & electron paramagnetic resonance \\
\hline ESI & electrospray ionization \\
\hline EtCN & propionitrile \\
\hline $\mathrm{Fc}$ & ferrocene \\
\hline FDP & flavodiiron protein \\
\hline FMN & flavin mononucleotide \\
\hline FprA & A-type flavoprotein \\
\hline$H$ & magnetic field strength, enthalpy (thermodynamics) \\
\hline HPh-bimp & 2,6-bis[[bis $(N$-methyl-4,5-diphenylimidazole-2-yl)methyl]- aminomethyl]-4-methylphenol \\
\hline $\mathrm{Hr}$ & hemerythrin \\
\hline$i \operatorname{Pr}$ & isopropyl \\
\hline IR & infra red \\
\hline $\mathrm{I} 2 \mathrm{M}$ & interaction of two $\mathrm{M}-\mathrm{O}$ units \\
\hline MB & Mössbauer \\
\hline $\mathrm{MeCN}$ & acetonitrile \\
\hline $\mathrm{MeOH}$ & methanol \\
\hline N-Et-hptb & $N, N, N^{\prime}, N^{\prime}$-tetrakis(2-(1-ethyl-benzimidazolylmethyl))-2-hydroxy-1,3-diaminopropane \\
\hline $\mathrm{NADH}$ & nicotinamide adenine dinucleotide (reduced form) \\
\hline NADP & nicotinamide adenine dinucleotide phosphate \\
\hline NHE & normal hydrogen electrode \\
\hline NMR & nuclear magnetic resonance \\
\hline NOR & nitric oxide reductase \\
\hline OEC & oxygen evolving complex \\
\hline PCET & proton coupled electron transfer \\
\hline ppm & parts per million \\
\hline PSI & photosystem I \\
\hline PSII & photosystem II \\
\hline PTFE & polytetrafluoroethylene \\
\hline$S$ & spin, entropy (thermodynamics) \\
\hline $\mathrm{SCE}$ & saturated calomel electrode \\
\hline
\end{tabular}




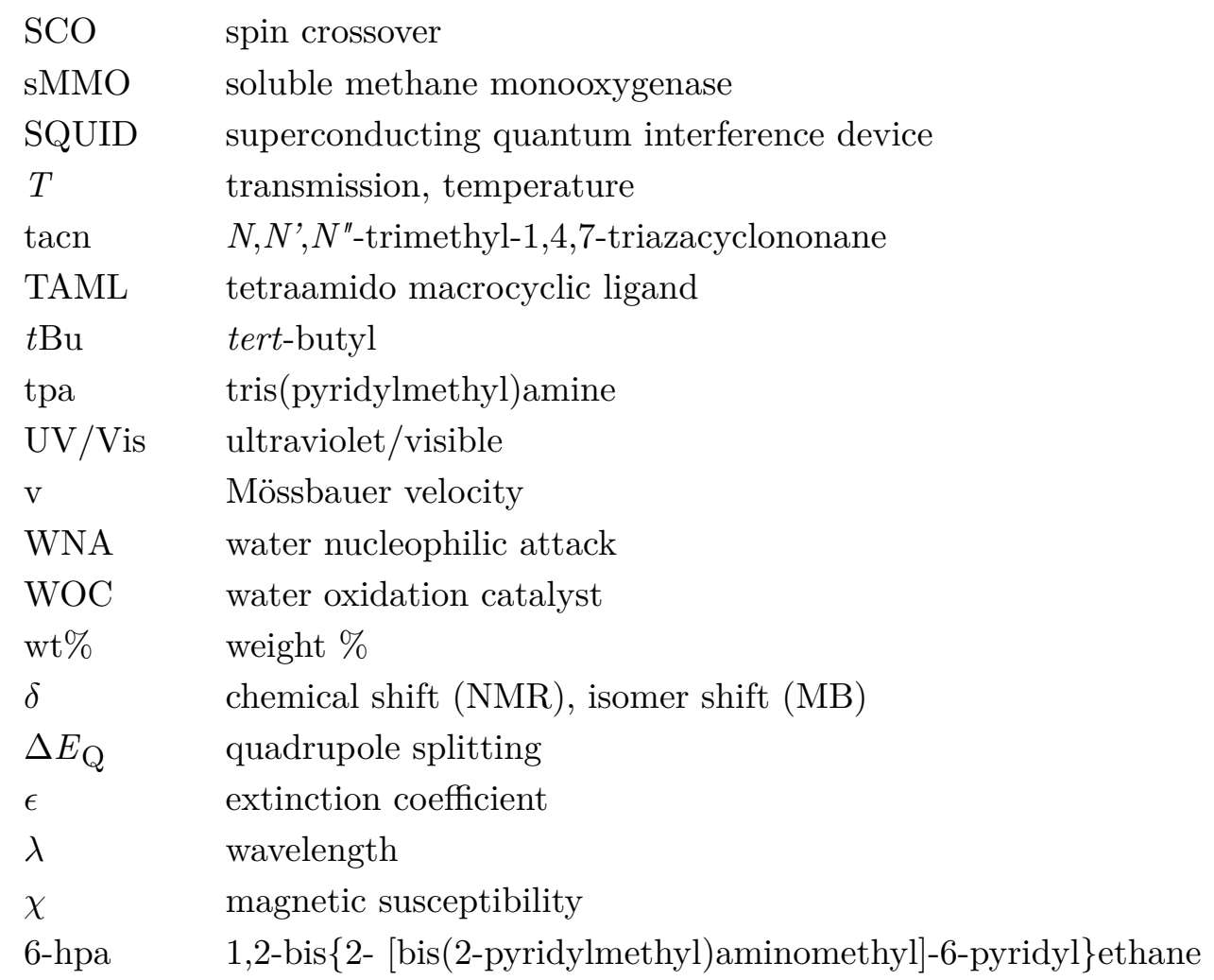




\subsection{Overview over Synthesized Complexes}

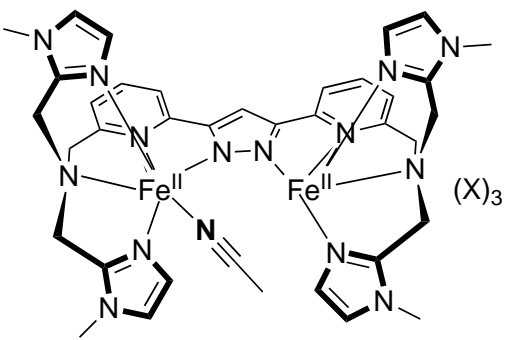

$\mathrm{X}=\mathrm{SO}_{3} \mathrm{CF}_{3} \mathbf{1 a}$

$$
\mathrm{X}=\mathrm{BF}_{4} \mathbf{1 b}
$$

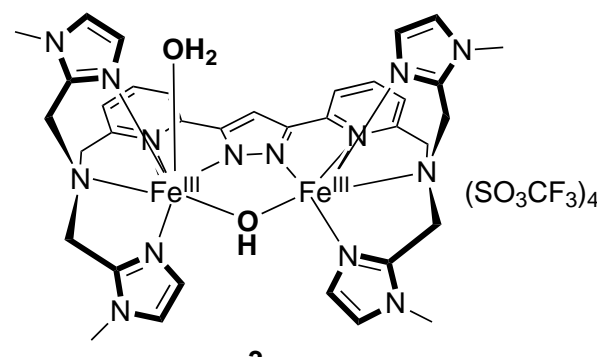

3
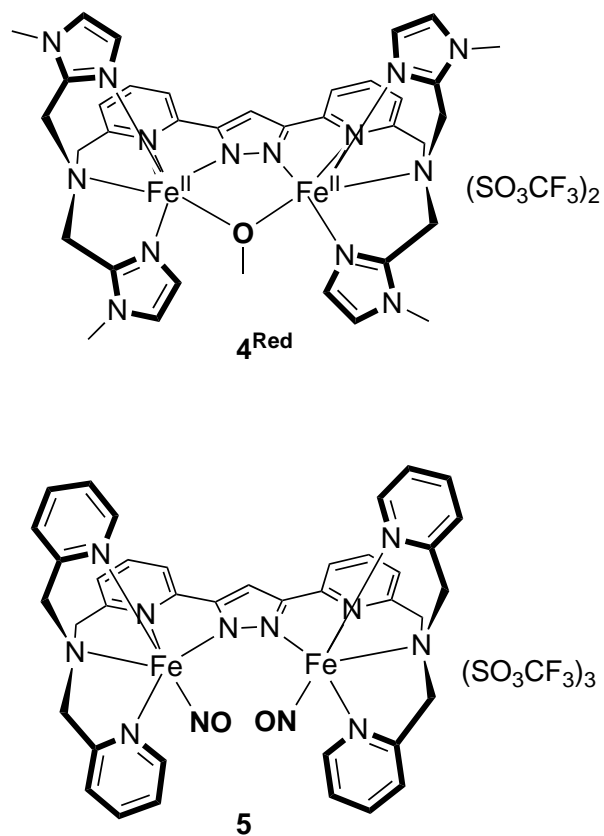

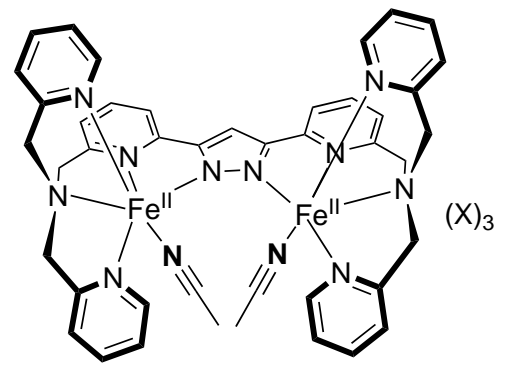

$\mathrm{X}=\mathrm{SO}_{3} \mathrm{CF}_{3} 2 \mathrm{a}$

$\mathrm{X}=\mathrm{BF}_{4} \mathbf{2 b}$
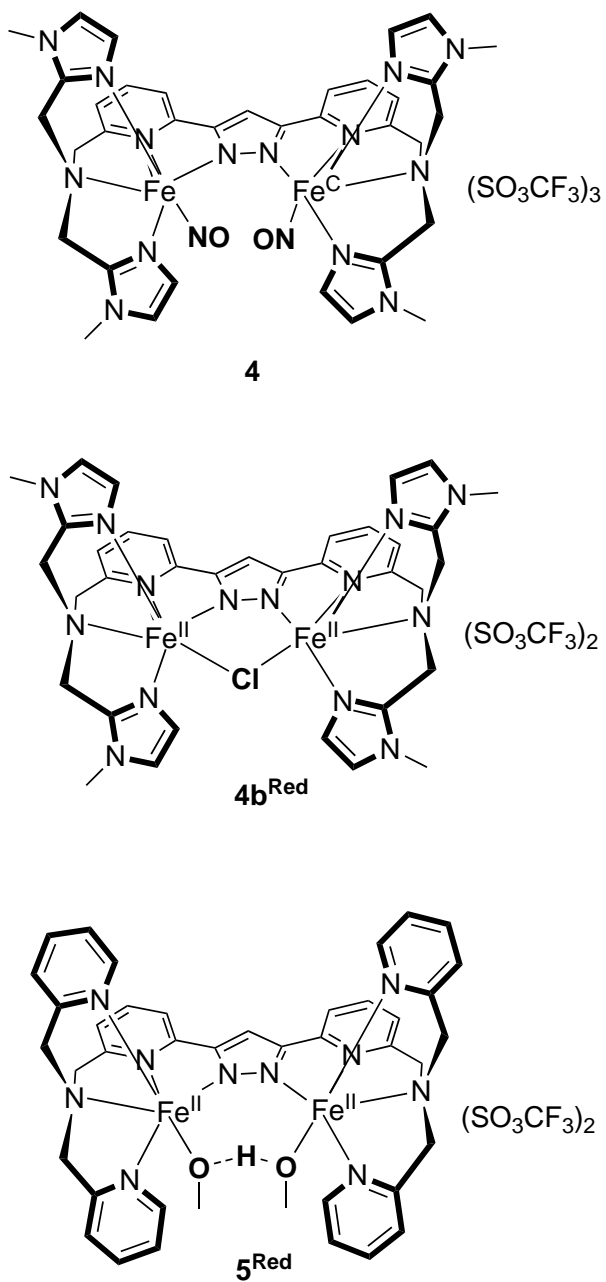

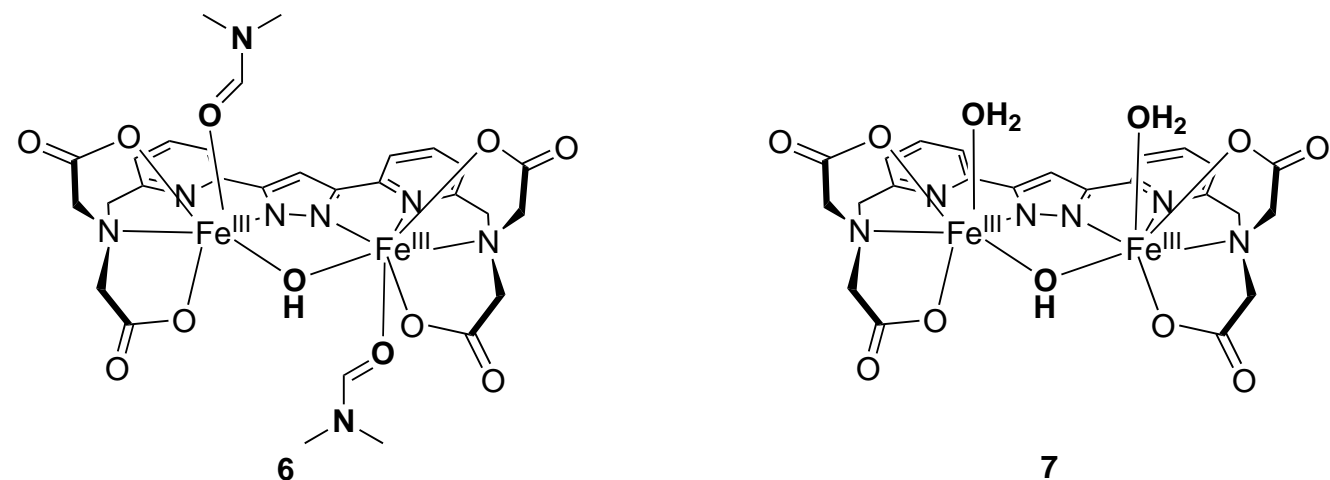

6

7

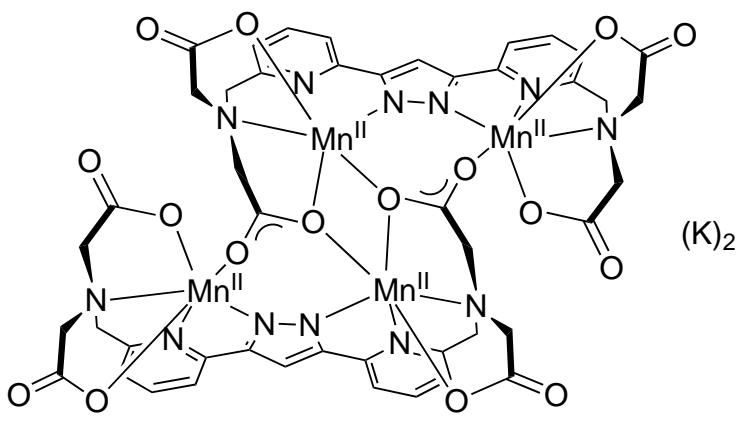

8 



\section{Bibliography}

[1] A. F. Hollemann, E. Wiberg, N. Wiberg, Lehrbuch der Anorganischen Chemie, 102th edition, Walter de Gruyter, 2007, pp. 1636.

[2] Römpp online, entry Eisen, 2006.

[3] G. Papanikolaou, K. Pantopoulos, Toxicol. Appl. Pharmacol. 2005, 202, 199-211.

[4] P. Gütlich, A. B. Gaspar, Y. Garcia, Beilstein J. Org. Chem. 2013, 9, 342-391.

[5] L. Que, W. B. Tolman, Nature 2008, 455, 333-340.

[6] I. Siewert, C. Limberg, Chem. A Eur. J. 2009, 15, 10316-10328.

[7] D. Lee, S. J. Lippard, Inorg. Chem. 2002, 41, 827-837.

[8] W. Nam, Acc. Chem. Res. 2007, 40, 465.

[9] Activation of Small Molecules: Organometallic and Bioinorganic Perspectives, Wiley-VCH GmbH \& Co KGaA, Weinheim, 2006.

[10] K. D. Kröncke, K. Fehsel, V. Kolb-Bachofen, Nitric Oxide 1997, 1, 107-120.

[11] C. T. Armstrong, D. W. Watkins, J. L. R. Anderson, Dalton Trans. 2013, 42, $3136-3150$.

[12] P. Ponka, Am. J. Med. Sci. 1999, 318, 241-256.

[13] I. G. Denisov, T. M. Makris, S. G. Sligar, I. Schlichting, Chem. Rev. 2005, 105, $2253-2277$.

[14] J. Rittle, M. T. Green, Science 2010, 330, 933-938.

[15] E. I. Solomon, A. Decker, N. Lehnert, Proc. Natl. Acad. Sci. U. S. A. 2003, 100, 3589-3594.

[16] P. C. A. Bruijnincx, G. van Koten, R. J. M. Klein Gebbink, Chem. Soc. Rev. 2008, 37, 2716-2744.

[17] M. Costas, M. P. Mehn, M. P. Jensen, L. Que, Chem. Rev. 2004, 104, 939-986.

[18] K. D. Koehntop, J. P. Emerson, L. Que, J. Biol. Inorg. Chem. 2005, 10, 87-93.

[19] E. G. Kovaleva, J. D. Lipscomb, Nat. Chem. Biol. 2008, 4, 186-193. 
[20] L. Que Jr, J. Chem. Soc., Dalt. Trans. 1997, 3933-3940.

[21] E. Y. Tshuva, S. J. Lippard, Chem. Rev. 2004, 104, 987-1012.

[22] P. Nordlund, H. Eklund, Curr. Opin. Struct. Biol. 1995, 5, 758-66.

[23] M. H. Sazinsky, S. J. Lippard, Acc. Chem. Res. 2006, 39, 558-566.

[24] G. Grüber, Structure 2015, 23, 805-806.

[25] R. E. Stenkamp, Chem. Rev. 1994, 94, 715-726.

[26] A. K. Shiemke, T. M. Loehr, J. Sanders-loehr, J. Am. Chem. Soc. 1986, 108, 69926996.

[27] M. Y. Okamura, I. M. Klotz, C. E. Johnson, M. R. C. Winter, R. J. P. Williams, Biochemistry 1969, 8, 1951-1958.

[28] P. E. Clark, J. Webb, Biochemistry 1981, 20, 4628-4632.

[29] A. C. Rosenzweig, C. A. Frederick, S. J. Lippard, P. Nordlund, Nature 1993, 366, $537-543$.

[30] A. C. Rosenzweig, P. Nordlund, P. M. Takahara, C. A. Frederick, S. J. Lippard, Chem. \& Biol. 1995, 2, 409-418.

[31] C. E. Tinberg, S. J. Lippard, Biochemistry 2009, 48, 12145-12158.

[32] C. E. Tinberg, S. J. Lippard, Acc. Chem. Res. 2011, 44, 280-288.

[33] M. Merkx, D. A. Kopp, M. H. Sazinsky, J. L. Blazyk, J. Müller, S. J. Lippard, Angew. Chem. Int. Ed. 2001, 40, 2782-2807.

[34] W. Wang, S. J. Lippard, J. Am. Chem. Soc. 2014, 136, 2244-2247.

[35] S. J. Lee, M. S. McCormick, S. J. Lippard, U.-S. Cho, Nature 2013, 494, 380-384.

[36] R. Banerjee, K. K. Meier, E. Münck, J. D. Lipscomb, Biochemistry 2013, 52, 43314342.

[37] R. Banerjee, Y. Proshlyakov, J. D. Lipscomb, D. A. Proshlyakov, Nature 2015, 518, 431-434.

[38] T. M. Makris, V. V. Vu, K. K. Meier, A. J. Komor, B. S. Rivard, E. Münck, L. Que, J. D. Lipscomb, J. Am. Chem. Soc. 2015, 137, 1608-1617.

[39] Y. S. Choi, H. Zhang, J. S. Brunzelle, S. K. Nair, H. Zhao, Proc. Natl. Acad. Sci. U. S. A. 2008, 105, 6858-6863.

[40] V. K. Korboukh, N. Li, E. W. Barr, J. M. Bollinger, C. Krebs, J. Am. Chem. Soc. 2009, 131, 13608-13609.

[41] A. J. Komor, B. S. Rivard, R. Fan, Y. Guo, J. Lawrence Que, J. D. Lipscomb, J Am. Chem. Soc 2016, 138, 7411-7421. 
[42] N. Li, V. K. Korboukh, C. Krebs, J. M. Bollinger, Proc. Natl. Acad. Sci. U. S. A. 2010, 107, 15722-15727.

[43] K. Garbett, D. Darnall, I. Klotz, R. Williams, Arch. Biochem. Biophys. 1969, 135, 419-434.

[44] K. E. Liu, D. Wang, B. H. Huynh, D. E. Edmondson, A. Salifoglou, S. J. Lippard, J. Am. Chem. Soc. 1994, 116, 7465-7466.

[45] K. E. Liu, A. M. Valentine, D. Wang, B. H. Huynh, D. E. Edmondson, A. Salifoglou, S. J. Lippard, J. Am. Chem. Soc. 1995, 117, 10174-10185.

[46] K. E. Liu, A. M. Valentine, D. Qui, D. E. Edmondson, E. H. Appelman, T. G. Spire, S. J. Lippard, J. Am. Chem. Soc. 1997, 119, 11134-11136.

[47] S. Friedle, E. Reisner, S. J. Lippard, Chem. Soc. Rev. 2010, 39, 2768-2779.

[48] W. H. Armstrong, S. J. Lippard, J. Am. Chem. Soc. 1983, 105, 4837-4838.

[49] W. H. Armstrong, A. Spool, G. C. Papaefthymiou, R. B. Frankel, S. J. Lippard, J. Am. Chem. Soc. 1984, 106, 3653-3667.

[50] W. H. Armstrong, S. J. Lippard, J. Am. Chem. Soc. 1984, 106(6), 4632-4633.

[51] P. Chaudhuri, K. Wieghardt, B. Nuber, J. Weiss, Angew. Chem. Int. Ed. 1985, 24, 778-779.

[52] J. A. R. Hartman, R. L. Rardin, P. Chaudhuri, K. Pohl, K. Wieghardt, B. Nuber, J. Weiss, G. C. Papaefthymiou, R. B. Frankel, S. J. Lippard, J. Am. Chem. Soc. 1987, 109, 7387-7396.

[53] C. He, A. M. Barrios, D. Lee, J. Kuzelka, R. M. Davydov, S. J. Lippard, J. Am. Chem. Soc. 2000, 122, 12683-12690.

[54] K. Wieghardt, K. Pohl, W. Gebert, Angew. Chem. Int. Ed. 1983, 22, 727.

[55] M. A. Holmes, I. Le Trong, S. Turley, L. C. Sieker, R. E. Stenkamp, J. Mol. Biol. 1991, 218, 583-593.

[56] M. A. Cranswick, K. K. Meier, X. Shan, A. Stubna, J. Kaizer, M. P. Mehn, E. Münck, L. Que, Inorg. Chem. 2012, 51, 10417-10426.

[57] T. Ookubo, H. Sugimoto, T. Nagayama, H. Masuda, T. Sato, K. Tanaka, Y. Maeda, H. kawa, Y. Hayashi, A. Uehara, M. Suzuki, J. Am. Chem. Soc. 1996, 118, 701-702.

[58] Y. Dong, S. Yan, V. Young, L. Que, Angew. Chem. Int. Ed. 1996, 991, 4-6.

[59] K. Kim, S. J. Lippard, J. Am. Chem. Soc. 1996, 118, 4914-4915.

[60] X. Zhang, H. Furutachi, S. Fujinami, S. Nagatomo, Y. Maeda, Y. Watanabe, T. Kitagawa, M. Suzuki, J. Am. Chem. Soc. 2005, 127, 826-827. 
[61] Y. Dong, S. Menage, J. Am. Chem. Soc. 1993, 115, 1851-1859.

[62] S. Menage, B. A. Brennan, C. Juarez-Garcia, E. Münck, L. Que, J. Am. Chem. Soc. 1990, 112, 6423-6425.

[63] A. C. Rosenzweig, Nature 2015, 518, 309-310.

[64] Y. Dong, Y. Zang, L. Shu, E. C. Wilkinson, J. Que, Lawrence, J. Am. Chem. Soc. 1997, 119, 12683-12684.

[65] G. Xue, D. Wang, R. De Hont, A. T. Fiedler, X. Shan, E. Münck, L. Que, Proc. Natl. Acad. Sci. 2007, 104, 20713-20718.

[66] G. Xue, A. Fiedler, Proc. Natl. Acad. Sci. 2008, 105, 20615-20620.

[67] G. Xue, R. De Hont, E. Münck, L. Que, Nat. Chem. 2010, 2, 400-405.

[68] G. Xue, A. Pokutsa, L. Que, J. Am. Chem. Soc. 2011, 133, 16657-16667.

[69] J. Kim, Y. Dong, E. Larka, L. Que, Inorg. Chem. 1996, 35, 2369-2372.

[70] A. R. McDonald, L. Que, Coord. Chem. Rev. 2013, 257, 414-428.

[71] M. Kodera, Y. Kawahara, Y. Hitomi, T. Nomura, T. Ogura, Y. Kobayashi, J. Am. Chem. Soc. 2012, 134, 13236-13239.

[72] M. Kodera, S. Ishiga, T. Tsuji, K. Sakurai, Y. Hitomi, Y. Shiota, P. K. Sajith, K. Yoshizawa, K. Mieda, T. Ogura, Chem. Eur. J. 2016, 22, 5924-5936.

[73] M. Kodera, M. Itoh, K. Kano, T. Funabiki, M. Reglier, Angew. Chem. Int. Ed. 2005, 44, 7104-7106.

[74] S. K. Lee, J. C. Nesheim, J. D. Lipscomb, J. Biol. Chem. 1993, 268, 21569-21577.

[75] S. K. Lee, B. G. Fox, W. A. Froland, J. D. Lipscomb, E. M. J, J. Am. Chem. Soc. 1993, 115, 6450-6451.

[76] L. Shu, J. C. Nesheim, K. Kauffmann, E. Münck, J. D. Lipscomb, L. Que, Science 1997, 275, 515-518.

[77] M. D. Bartberger, W. Liu, E. Ford, K. M. Miranda, C. Switzer, J. M. Fukuto, P. J. Farmer, D. A. Wink, K. N. Houk, Proc. Natl. Acad. Sci. U. S. A. 2002, 99, 10958-10963.

[78] P. R. Gardner, L. a. Martin, D. Hall, A. M. Gardner, Free Radic. Biol. Med. 2001, 31, 191-204.

[79] I. M. Wasser, S. de Vries, P. Moënne-Loccoz, I. Schröder, K. D. Karlin, Chem. Rev. 2002, 102, 1201-1234.

[80] J. A. McCleverty, Chem. Rev. 2004, 104, 403-418.

[81] A. L. Feig, M. T. Bautista, S. J. Lippard, Inorg. Chem. 1996, 35, 6892-6898. 
[82] J. H. Enemark, R. D. Feltham, Coord. Chem. Rev. 1974, 13, 339-406.

[83] T. C. Berto, A. L. Speelman, S. Zheng, N. Lehnert, Coord. Chem. Rev. 2013, 257, $244-259$.

[84] P. Moënne-Loccoz, Nat. Prod. Rep. 2007, 24, 610-620.

[85] V. K. K. Praneeth, F. Paulat, T. C. Berto, S. D. George, D. Kiel, J. Am. Chem. Soc. 2008, 130, 15288-15303.

[86] J. P. Collman, Y. Yang, A. Dey, R. a. Decréau, S. Ghosh, T. Ohta, E. I. Solomon, Proc. Natl. Acad. Sci. U. S. A. 2008, 105, 15660-15665.

[87] L. E. Goodrich, F. Paulat, V. K. K. Praneeth, N. Lehnert, Inorg. Chem. 2010, 49, 6293-6316.

[88] T. C. Berto, M. B. Ho, Y. Murata, K. B. Landenberger, E. E. Alp, J. Zhao, N. Lehnert, J. Am. Chem. Soc. 2011, 133, 16714-16717.

[89] L. E. Goodrich, S. Roy, E. E. Alp, J. Zhao, M. Y. Hu, N. Lehnert, Inorg. Chem. 2013, 52, 7766-7780.

[90] A. L. Speelman, N. Lehnert, Acc. Chem. Res. 2014, 47, 1106-1116.

[91] C. V. Romão, J. B. Vicente, P. T. Borges, C. Frazão, M. Teixeira, J. Biol. Inorg. Chem. 2016, 21, 39-52.

[92] R. Silaghi-Dumitrescu, E. D. Coulter, A. Das, L. G. Ljungdahl, G. N. L. Jameson, B. H. Huynh, D. M. Kurtz, Biochemistry 2003, 42, 2806-2815.

[93] C. Frazão, G. Silva, C. M. Gomes, P. Matias, R. Coelho, L. Sieker, S. Macedo, M. Y. Liu, S. Oliveira, M. Teixeira, A. V. Xavier, C. Rodrigues-Pousada, M. A. Carrondo, J. Le Gall, Nat. Struct. Biol. 2000, 7, 1041-1045.

[94] R. Silaghi-Dumitrescu, D. Kurtz, Biochemistry 2005, 44, 6492-6501.

[95] D. M. Kurtz, Jr., Dalton Trans. 2007, 4115-4121.

[96] S. Khatua, A. Majumdar, J. Inorg. Biochem. 2015, 142, 145-153.

[97] A. A. A. Attia, R. Silaghi-Dumitrescu, Eur. J. Inorg. Chem. 2014, 6061-6065.

[98] J. D. Caranto, A. Weitz, M. P. Hendrich, D. M. Kurtz, J. Am. Chem. Soc. 2014, 136, 7981-7992.

[99] J. D. Caranto, A. Weitz, N. Giri, M. P. Hendrich, D. M. Kurtz, Biochemistry 2014, 53, 5631-5637.

[100] T. Suzuki, H. Tanaka, Y. Shiota, P. K. Sajith, Y. Arikawa, K. Yoshizawa, Inorg. Chem. 2015, 54, 7181-7191.

[101] D. Lionetti, G. de Ruiter, T. Agapie, J. Am. Chem. Soc. 2016, 138, 5008-5011. 
[102] Y. Jiang, T. Hayashi, H. Matsumura, L. H. Do, A. Majumdar, S. J. L. P. MoenneLoccoz, S. J. Lippard, P. Moënne-Loccoz, J. Am. Chem. Soc. 2015, 136, 1252412527.

[103] S. Zheng, T. C. Berto, E. W. Dahl, M. B. Hoffman, A. L. Speelman, N. Lehnert, J. Am. Chem. Soc. 2013, 135, 4902-4905.

[104] W. Zhang, J. Liu, H. Zhu, W. Gao, L. Sun, Synth. Commun. 2007, 37, 3393-3402.

[105] S. Fischer, Synthese neuartiger pyrazolbasierter Liganden und deren Übergangsmetallkomplexe, PhD Thesis, Georg August Universität Göttingen, 2011.

[106] A. von Zelewsky, Stereochemistry of Coordination Compounds, John Wiley \& Sons, 1996, pp. 177.

[107] R. D. Hancock, J. Chem. Educ. 1992, 69, 615-621.

[108] S. Trofimenko, Chem. Rev. 1972, 72, 497-509.

[109] R. Mukherjee, Coord. Chem. Rev. 2000, 203, 151-218.

[110] G. Monica, G. Ardizzoia, Prog. Inorg. Chem. 1997, 46, 151-283.

[111] W. Kaim, B. Schwederski, Bioanorganische Chemie, 4th edition, Vieweg+Teubner, 2005.

[112] R. H. Beer, W. B. Tolman, S. G. Bott, S. J. Lippard, Inorg. Chem. 1991, 30, 2082-2092.

[113] B. Burger, S. Dechert, C. Große, S. Demeshko, F. Meyer, Chem. Commun. 2011, 47, 10428-10430.

[114] J. Du Bois, T. J. Mizoguchi, S. J. Lippard, Coord. Chem. Rev. 2000, 200-202, $443-485$.

[115] J. Klingele, S. Dechert, F. Meyer, Coord. Chem. Rev. 2009, 253, 2698-2741.

[116] J. Ackermann, F. Meyer, H. Pritzkow, Inorg. Chim. Acta 2004, 357, 3703-3711.

[117] K. E. Dalle, F. Meyer, Eur. J. Inorg. Chem. 2015, 3391-3405.

[118] S. Neudeck, S. Maji, I. López, S. Meyer, F. Meyer, A. Llobet, J. Am. Chem. Soc. 2014, 136, 24-27.

[119] S. Neudeck, S. Maji, I. López, S. Dechert, J. Benet-Buchholz, A. Llobet, F. Meyer, Inorg. Chem. 2016, 55, 2508-2521.

[120] A. C. Sander, A. Schober, S. Dechert, F. Meyer, Eur. J. Inorg. Chem. 2015, 43484353.

[121] A. C. Sander, S. Maji, L. Francàs, T. Böhnisch, S. Dechert, A. Llobet, F. Meyer, ChemSusChem 2015, 8, 1697-1702. 
[122] B. Schneider, S. Demeshko, S. Dechert, F. Meyer, Angew. Chem. Int. Ed. 2010, 49, 9274-9277.

[123] M. Steinert, B. Schneider, S. Dechert, S. Demeshko, F. Meyer, Angew. Chem. Int. Ed. 2014, 53, 6135-6139.

[124] M. Steinert, B. Schneider, S. Dechert, S. Demeshko, F. Meyer, Inorg. Chem. 2016, $55,2363-2373$.

[125] X. Sala, S. Maji, R. Bofill, J. García-Antón, L. Escriche, A. Llobet, Acc. Chem. Res. 2014, 47, 504-516.

[126] B. Burger, Structure, Dynamics and Bioinspired Reactivity of Novel Pyrazolate-based Diiron Complexes, PhD Thesis, Georg August Universität Göttingen, 2012.

[127] B. Burger, S. Demeshko, E. Bill, S. Dechert, F. Meyer, Angew. Chem. Int. Ed. 2012, $51,10045-10049$.

[128] N. Kindermann, Diiron and Dicopper Complexes with Pyrazolate / tacn Hybrid Ligands for Small Molecule Activation, PhD Thesis, Georg August Universität Göttingen, 2015.

[129] K. Oberhausen, J. Richardson, R. Buchanan, W. Pierce, Polyhedron 1989, 8, 659668 .

[130] S. Wöckel, Synthetic Models for Dizinc Metallohydrolases, PhD Thesis, Georg August Universität Göttingen, 2011.

[131] Y. Liu, R. Xiang, X. Du, Y. Ding, B. Ma, Chem. Commun. 2014, 50, 12779-12782.

[132] A. Schober, Eisenkomplexe neuer Pyrazol-Kompartimentliganden für die bioinspirierte Oxidationskatalyse, Master Thesis, Georg August Universität Göttingen, 2012.

[133] S. Fischer, S. Demeshko, S. Dechert, F. Meyer, Z. Anorg. Allg. Chem. 2012, 638, $621-627$.

[134] M. Steinert, Defektgitter und Gitterkomplexe-Einflüsse von Gegenionen und Lösungsmitteln auf ihre magnetischen Eigenschaften, PhD Thesis, Georg August Universität Göttingen, 2015.

[135] S. Samanta, S. Demesko, S. Dechert, F. Meyer, Angew. Chem. Int. Ed. 2015, 54, $583-587$.

[136] P. Gütlich, Y. Garcia, H. A. Goodwin, Chem. Soc. Rev. 2000, 29, 419-427.

[137] A. Bousseksou, G. Molnár, J. A. Real, K. Tanaka, Coord. Chem. Rev. 2007, 251, $1822-1833$.

[138] P. Gütlich, H. A. Goodwin, Spin Crossover in Transition Metal Compounds I -III, Topics in Current Chemistry, Springer Berlin Heidelberg, 2004. 
[139] A. Bousseksou, G. Molnár, L. Salmon, W. Nicolazzi, Chem. Soc. Rev. 2011, 40, 3313-3335.

[140] P. Gütlich, A. Hauser, H. Spiering, Angew. Chem. Int. Ed. 1994, 33, 2024-2054.

[141] A. W. Addison, T. N. Rao, J. Reedijk, J. van Rijn, G. Verschoor, J. Chem. Soc. Dalt. Trans. 1984, 1349-1356.

[142] R. Heintz, J. Smith, Inorg. Synth. 2002, 33, 75-83.

[143] M. M. Dîrtu, A. Rotaru, D. Gillard, J. Linares, E. Codjovi, B. Tinant, Y. Garcia, Inorg. Chem. 2009, 48, 7838-7852.

[144] M. M. Dîrtu, C. Neuhausen, A. D. Naik, A. Rotaru, L. Spinu, Y. Garcia, Inorg. Chem. 2010, 49, 5723-5736.

[145] O. Roubeau, J. M. Alcazar Gomez, E. Balskus, J. J. a. Kolnaar, J. G. Haasnoot, J. Reedijk, New J. Chem. 2001, 25, 144-150.

[146] C. Grunert, S. Reiman, H. Spiering, J. Kitchen, S. Brooker, P. Gütlich, Angew. Chem. Int. Ed. 2008, 47, 2997-2999.

[147] J. Olguín, S. Brooker, Coord. Chem. Rev. 2011, 255, 203-240.

[148] R. Kulmaczewski, J. Olguín, J. a. Kitchen, H. L. C. Feltham, G. N. L. Jameson, J. L. Tallon, S. Brooker, J. Am. Chem. Soc. 2014, 136, 878-81.

[149] K. Nakano, N. Suemura, S. Kawata, A. Fuyuhiro, T. Yagi, S. Nasu, S. Morimoto, S. Kaizaki, Dalton Trans. 2004, 982-988.

[150] H. Toftlund, Monatsh. Chem. 2001, 132, 1260-1277.

[151] K. Bryliakov, E. Duban, E. Talsi, Eur. J. Inorg. Chem. 2005, 2005, 72-76.

[152] D. W. Blakesley, S. C. Payne, K. S. Hagen, Inorg. Chem. 2000, 39, 1979-1989.

[153] W. Klaui, W. Eberspach, P. Gütlich, Inorg. Chem. 1987, 26, 3977-3982.

[154] H.-J. Lin, D. Siretanu, D. A. Dickie, D. Subedi, J. J. Scepaniak, D. Mitcov, R. Clérac, J. M. Smith, J. Am. Chem. Soc. 2014, 136, 13326-13332.

[155] D. Wiedemann, A. Grohmann, Dalton Trans. 2014, 43, 2406-2417.

[156] K. Ralph, W. Florida, E. Schubert, J. Chem. Educ. 1992, 1958.

[157] J. Wang, G. Li, Q. S. Li, Y. Xie, R. B. King, Polyhedron 2012, 47, 165-172.

[158] M. L. Kuznetsov, Russ. Chem. Rev. 2007, 71, 265-282.

[159] B. J. Wallar, J. D. Lipscomb, Chem. Rev. 1996, 96, 2625-2658.

[160] D. M. Kurtz Jr, Chem. Rev. 1990, 90, 585-606. 
[161] S. Hong, K. D. Sutherlin, J. Park, E. Kwon, M. A. Siegler, E. I. Solomon, W. Nam, Nat. Commun. 2014, 5, 5440.

[162] C. W. Chiang, S. T. Kleespies, H. D. Stout, K. K. Meier, P. Y. Li, E. L. Bominaar, L. Que, E. Münck, W. Z. Lee, J. Am. Chem. Soc. 2014, 136, 10846-10849.

[163] F. Oddon, Y. Chiba, J. Nakazawa, T. Ohta, T. Ogura, S. Hikichi, Angew. Chem. Int. Ed. 2015, 54, 7336-7339.

[164] A. Mukherjee, M. a. Cranswick, M. Chakrabarti, T. K. Paine, K. Fujisawa, E. Münck, L. Que, Inorg. Chem. 2010, 49, 3618-28.

[165] J. Jullien, G. Juhász, P. Mialane, E. Dumas, C. R. Mayer, J. Marrot, E. Rivière, E. L. Bominaar, E. Münck, F. Sécheresse, Inorg. Chem. 2006, 45, 6922-7.

[166] B. A. Brennan, Q. Chen, C. Juarez-Garcia, A. E. True, C. J. O'Connor, L. Que, Inorg. Chem. 1991, 30, 1937-1943.

[167] A. L. Smith, W. E. Keller, H. L. Johnston, J. Chem. Phys. 1951, 19, 189-192.

[168] M. Römelt, S. Ye, F. Neese, Inorg. Chem. 2009, 48, 784-785.

[169] T. Moriya, Phys. Rev. 1960, 120, 91-98.

[170] I. Dzyaloshinsky, J. Phys. Chem. Solids 1958, 4, 241-255.

[171] S. Herzog, Spin-orbit effects in electronic transport through a molecular dimer, $\mathrm{PhD}$ Thesis, RWTH Aachen, 2009.

[172] K. E. Kauffmann, C. V. Popescu, Y. Dong, J. D. Lipscomb, L. Que, E. Münck, J. Am. Chem. Soc. 1998, 120, 8739-8746.

[173] P. M. Levy, Phys. Rev. Lett. 1968, 20(24).

[174] E. Bill, julX -Simulation of Molecular Magnetic Data, Max Planck Institute for Chemical Energy Conversion, Mülheim/Ruhr, Germany.

[175] C. A. Brown, M. a. Pavlosky, T. E. Westre, Y. Zhang, B. Hedman, K. O. Hodgson, E. I. Solomon, J. Am. Chem. Soc. 1995, 117, 715-732.

[176] C. Hauser, T. Glaser, E. Bill, J. Am. Chem. Soc. 2000, 122, 4352-4365.

[177] R. García Serres, C. A. Grapperhaus, E. Bothe, E. Bill, T. Weyhermüller, F. Neese, K. Wieghardt, J. Am. Chem. Soc. 2004, 126, 5138-5153.

[178] N. G. Connelly, W. E. Geiger, Chem. Rev. 1996, 96, 877-910.

[179] R. Pulukkody, M. Y. Darensbourg, Acc. Chem. Res. 2015, 48, 2049-2058.

[180] M.-L. Tsai, C.-C. Tsou, W.-F. Liaw, Acc. Chem. Res. 2015, 48, 1184-1193.

[181] L. Schlapbach, A. Züttel, Nature 2001, 414, 353-358. 
[182] S. Styring, Faraday Discuss. 2012, 155, 357-376.

[183] N. S. Lewis, D. G. Nocera, Proc. Natl. Acad. Sci. 2006, 103, 15729-15735.

[184] S. Berardi, S. Drouet, L. Francàs, C. Gimbert-Suriñach, M. Guttentag, C. Richmond, T. Stoll, A. Llobet, Chem. Soc. Rev. 2014, 43, 7501-7519.

[185] V. Artero, M. Fontecave, Chem. Soc. Rev. 2013, 42, 2338-2356.

[186] J. Barber, Chem. Soc. Rev. 2009, 38, 185-196.

[187] R. H. Crabtree, Energy Production and Storage: Inorganic Chemical Strategies for a Warming World, John Wiley \& Sons, 2013.

[188] J. S. Vrettos, G. W. Brudvig, Phil. Trans. R. Soc. Lond. B 2002, 357, 1395-1405.

[189] Y. Umena, K. Kawakami, J.-R. Shen, N. Kamiya, Nature 2011, 473, 55-60.

[190] C. Kupitz, S. Basu, I. Grotjohann, R. Fromme, N. A. Zatsepin, K. N. Rendek, M. S. Hunter, R. L. Shoeman, T. A. White, D. Wang, D. James, J.-H. Yang, D. E. Cobb, B. Reeder, R. G. Sierra, H. Liu, A. Barty, A. L. Aquila, D. Deponte, R. A. Kirian, S. Bari, J. J. Bergkamp, K. R. Beyerlein, M. J. Bogan, C. Caleman, T.-C. Chao, C. E. Conrad, K. M. Davis, H. Fleckenstein, L. Galli, S. P. Hau-Riege, S. Kassemeyer, H. Laksmono, M. Liang, L. Lomb, S. Marchesini, A. V. Martin, M. Messerschmidt, D. Milathianaki, K. Nass, A. Ros, S. Roy-Chowdhury, K. Schmidt, M. Seibert, J. Steinbrener, F. Stellato, L. Yan, C. Yoon, T. A. Moore, A. L. Moore, Y. Pushkar, G. J. Williams, S. Boutet, R. B. Doak, U. Weierstall, M. Frank, H. N. Chapman, J. C. H. Spence, P. Fromme, Nature 2014, 513, 261-265.

[191] N. Cox, M. Retegan, F. Neese, D. A. Pantazis, A. Boussac, W. Lubitz, Science 2014, 345, 804-808.

[192] J. D. Blakemore, R. H. Crabtree, G. W. Brudvig, Chem. Rev. 2015, 115, 1297413005 .

[193] A. R. Parent, K. Sakai, ChemSusChem 2014, 7, 2070-2080.

[194] P. Du, R. Eisenberg, Energy Environ. Sci. 2012, 5, 6012-6021.

[195] W. C. Ellis, C. T. Tran, M. A. Denardo, A. Fischer, A. D. Ryabov, T. J. Collins, J. Am. Chem. Soc. 2009, 131, 18052-18053.

[196] A. D. Ryabov, T. J. Collins, Adv. Inorg. Chem. 2009, 61, 471-521.

[197] W. C. Ellis, N. D. McDaniel, S. Bernhard, T. J. Collins, J. Am. Chem. Soc. 2010, 132, 10990-10991.

[198] V. Polshin, D. L. Popescu, A. Fischer, A. Chanda, D. C. Horner, E. S. Beach, J. Henry, Y. L. Qian, C. P. Horwitz, G. Lente, I. Fabian, E. Münck, E. L. Bominaar, A. D. Ryabov, T. J. Collins, J. Am. Chem. Soc. 2008, 130, 4497-4506. 
[199] A. Ghosh, A. D. Ryabov, S. M. Mayer, D. C. Horner, D. E. Prasuhn, S. Sen Gupta, L. Vuocolo, C. Culver, M. P. Hendrich, C. E. F. Rickard, R. E. Norman, C. P. Horwitz, T. J. Collins, J. Am. Chem. Soc. 2003, 125, 12378-12379.

[200] C. Panda, J. Debgupta, D. Díaz Díaz, K. K. Singh, S. Sen Gupta, B. B. Dhar, J. Am. Chem. Soc. 2014, 136, 12273-82.

[201] Z. Codolà, L. Gómez, S. T. Kleespies, L. Que Jr, M. Costas, J. Lloret-Fillol, Nat. Commun. 2015, 6, 1-9.

[202] M. M. Najafpour, A. N. Moghaddam, D. J. Sedigh, M. Hołyńska, Catal. Sci. Technol. 2014, 4, 30-33.

[203] L. D. Wickramasinghe, R. Zhou, R. Zong, P. Vo, K. J. Gagnon, R. P. Thummel, J. Am. Chem. Soc. 2015, 137, 13260-13263.

[204] P. Gütlich, Z. Anorg. Allg. Chem. 2012, 638, 15-43.

[205] C. Costentin, J.-M. Savéant, ChemElectro Chem 2014, 1, 1226-1236.

[206] R. H. Crabtree, Chem. Rev. 2012, 112, 1536-1554.

[207] A. R. Parent, R. H. Crabtree, G. W. Brudvig, Chem. Soc. Rev. 2013, 42, 2247-2252.

[208] W. A. A. Arafa, M. D. Kärkäs, B.-L. Lee, T. Åkermark, R.-Z. Liao, H.-M. Berends, J. Messinger, P. E. M. Siegbahn, B. Åkermark, Phys. Chem. Chem. Phys. 2014, 16, $11950-64$.

[209] E. S. Rountree, B. D. McCarthy, T. T. Eisenhart, J. L. Dempsey, Inorg. Chem. 2014, 53, 9983-10002.

[210] K. S. Hagen, Inorg. Chem. 2000, 39, 5867-5869.

[211] G. M. Sheldrick, Acta Crystallogr. A. 2008, 64, 112-22.

[212] X-RED, STOE \& CIE GmbH, Darmstadt, Germany, 2002.

[213] E. Bill, Mfit, Max Planck Institute for Chemical Energy Conversion, Mülheim/Ruhr, Germany.

[214] W. Haberditzl, Angew. Chem. Int. Ed. 1966, 5, 288-298.

[215] G. A. Bain, J. F. Berry, J. Chem. Educ. 2008, 85, 532-536.

[216] F. Neese, WIREs Comput. Mol. Sci. 2012, 2, 73-78.

[217] A. Schäfer, H. Horn, R. Ahlrichs, J. Chem. Phys. 1992, 97, 2571-2577.

[218] F. Weigend, R. Ahlrichs, Phys. Chem. Chem. Phys. 2005, 7, 3297-305.

[219] P. Gütlich, E. Bill, A. X. Trautwein, Mössbauer Spectroscopy and Transition Metal Chemistry, Springer Verlag, Berlin, Heidelberg, 2011. 
[220] R. L. Mössbauer, Z. Phys. 1958, 151, 124-143.

[221] R. L. Mössbauer, W. H. Wiedemann, Z. Phys. 1960, 159, 33-48.

[222] R. L. Mössbauer, Angew. Chem. Int. Ed. 1971, 10, 462-472.

[223] J. H. Rodriguez, Y.-M. Xia, P. G. Debrunner, J. Am. Chem. Soc. 1999, 121, 78467863.

[224] V. K. K. Praneeth, F. Neese, N. Lehnert, Inorg. Chem. 2005, 44, 2570-2572.

[225] A. L. Speelman, B. Zhang, A. Silakov, K. M. Skodje, E. E. Alp, J. Zhao, M. Y. Hu, E. Kim, C. Krebs, N. Lehnert, Inorg. Chem. 2016, 55, 5485-5501.

[226] M. McElfresh, Fundamentals of Magnetism and Magnetic Measurements Featuring Quantum Design's Magnetic Property Measurement system, Quantum Design, Supplementary Material, 1994, 1-38.

[227] Jülicher SQUID GmbH, JSQ-Tutorial für Benutzer von rf-SQUIDs, 2005.

[228] M. McElfresh, S. Li, R. Sager, Effects of Magnetic Field Uniformity on the Measurement of Superconducting Samples, Quantum Design, Supplementary Material, 2000, 1-11.

[229] P. W. Atkins, J. De Paula, Physikalische Chemie, Wiley-VCH, Weinheim, 2006.

[230] O. Kahn, Molecular Magnetism, VCH Publishers Inc., New York, 1993.

[231] S. Demeshko, Vorlesungsskript Methoden der Chemie III, Georg August Universität, Göttingen, 2010.

[232] B. Weber, Skript zur Vorlesung Molekulare Magneten, Universität München, 2009.

[233] J. I. Hoppe, J. Chem. Educ. 1972, 49, 505.

[234] P. W. Anderson, Phys. Rev. 1959, 115, 2-13.

[235] O. Kahn, Inorg. Chim. Acta 1982, 62, 3-14. 


\section{Danksagung}

Zunächst möchte ich herzlich meinem Doktorvater Prof. Dr. Franc Meyer danken für die interessante und umfangreiche Themenstellung dieser Arbeit und die nette Betreuung während der letzten Jahre. Auch bedanken möchte ich mich für die Möglichkeit an verschiedenen Konferenzen teilzunehmen und die hilfreichen Diskussionen in monatlichen Kleingruppenseminaren, welche sicherlich zum Gelingen dieser Arbeit beigetragen haben.

Dr. Inke Siewert möchte ich für die Übernahme des Korreferats danken, sowie für Anregungen und eine offene Tür bei Fragen und Problemen.

Sehr geschätzt habe ich das Erlernen des eigenständigen Messen und Lösen von Kristallstrukturen. Hierfür danke ich ganz besonders Dr. Sebastian Dechert für die Einführung in die Feinheiten zur Bedienung des Röntgendiffraktometers. Ihm sowie Nicole Kindermann gilt ein besonderer Dank für die Gedult und unermüdliche Unterstützung bei der Auswertung der Daten.

Dr. Serhiy Demeshko danke ich für das Messen und die Hilfe bei der Interpretation von Mössbauer und SQUID Daten. Des Weiteren, danke ich Christine Schiewer, Eleonora Feretti und Claudia Kupper für das Aufnehmen einiger Datensätze.

Eine interessante Zeit waren für mich auch die beiden Monate Forschungsaufenthalt in Ann Arbor, Michigan in der Arbeitsgruppe von Prof. Dr. Nicolai Lehnert. Bei Prof. Lehnert möchte ich mich für die Gastfreundschaft und Diskussionen bezüglich der durchgeführten Messungen bedanken. Für Hilfe im Labor und Tipps zur Durchführung einiger Messungen danke ich Ashley McQuarters und Amy Speelman. Der gesamten Arbeitsgruppe Lehnert, insbesondere Ashley danke ich für eine schöne Zeit auch außerhalb der Uni.

Andreas Schwarz und Jörg Teichgräber danke ich für technische Unterstützung während der gesamten Zeit im Labor. Andreas danke ich außerdem für das Nachziehen einiger Ligandvorstufen und Jörg für das Messen von Cyclovoltammogrammen.

Für das Messen der Liganden NMR-Spektren danke ich Ralf Schöne. Für Unterstützung bei der Durchführung temperaturabhängiger NMR Messungen danke ich Dennis Manz. Dem analytischen Labor des Instituts für Anorganische Chemie danke ich für das Messen von Elementaranalysen. Dr. Claudia Stückl, Hanna Steininger, Petra Unger sowie 
Britta Müller danke ich für die Hilfe in allen administrativen und organisatorischen Angelegenheiten.

Bei Thorsten Stolper bedanke ich mich für hilfreiche Ratschläge bezüglich einiger DFT Rechnungen.

Ein ganz großer Dank gilt meinen beiden Laborkolleginnen Nicole Kindermann und Vandana Goswami für die schöne Zeit zusammen, viele nette Diskussionen über Chemie und auch viele andere Dinge, sowie unsere Treffen auch außerhalb der Uni. Ich habe die Zeit sehr genossen. Auch Felix Klinke und Alexander Brinkmeier aus unserem "Kooperationslabor" danke ich für die nette Nachbarschaft mit vielen Diskussionen und Gesprächen.

Die Wartung von verschiedenen Großgeräten, sowie der Gloveboxen sowie das Destillieren von Lösungsmitteln ist oft sehr zeitaufwendig. In diesem Zusammenhang möchte ich ganz besonders Alex Gers-Barlag, Felix Klinke, Kai Kalz, Alexander Brinkmeier, Nicole Kindermann, Oliver Mitevski, Anastasia Vogel und Pierre Goursot danken.

Für die letzten Analysen war ich auf viele helfende Hände angewiesen. Ich möchte mich dafür sehr bei Marten Seeba, Nicole Kindermann, Alexander Brinkmeier, Alex Gers-Barlag, Can-Jerome Günes, Stefan Resch, Christine Schiewer und Dr. Sebastian Dechert bedanken.

Für das Korrekturlesen dieser Arbeit gilt mein Dank ganz besonders Nicole Kindermann, Christine Schiewer, Alexander Brinkmeier, Merle Kügler, Dr. Serhiy Demeshko, Stefan Resch und Sebastian Smolne.

Für eine schöne Zeit in als auch außerhalb der Uni, sowie viele lustige Gespräche bei einer Tasse Kaffee danke ich Nicole, Vandana, Alex, Olli, Anastasia, Sebastian, Marten und Felix. Dem gesamten Arbeitskreis Meyer danke ich außerdem für die nette und offene Arbeitsatmosphäre.

Dem Graduiertenkolleg IRTG1422: Metal Sites in Biomolecules: Structures, Regulations and Mechanisms, danke ich für einen Großteil der Finanzierung während der letzten drei Jahre sowie die Übernahme der Kosten für Workshops, Kurse und Konferenzen.

Meinen Eltern und Geschwistern danke ich für die finanzielle sowie moralische Unterstützung während des gesamten Studiums.

Meinem Freund Sebastian danke ich für seine liebevolle Hilfe während des Studiums und für viele motivierende und aufmunternde Worte gerade in den letzten Wochen. 